\title{
Under [re]construction : neurocognitive correlates of pseudo-memories
}

\author{
Citation for published version (APA):
}

Peters, M. J. V. (2007). Under [re]construction : neurocognitive correlates of pseudo-memories. [Doctoral Thesis, Maastricht University]. Datawyse / Universitaire Pers Maastricht. https://doi.org/10.26481/dis.20070607mp

Document status and date:

Published: 01/01/2007

DOI:

10.26481/dis.20070607mp

Document Version:

Publisher's PDF, also known as Version of record

\section{Please check the document version of this publication:}

- A submitted manuscript is the version of the article upon submission and before peer-review. There can be important differences between the submitted version and the official published version of record.

People interested in the research are advised to contact the author for the final version of the publication, or visit the DOI to the publisher's website.

- The final author version and the galley proof are versions of the publication after peer review.

- The final published version features the final layout of the paper including the volume, issue and page numbers.

Link to publication

\footnotetext{
General rights rights.

- You may freely distribute the URL identifying the publication in the public portal. please follow below link for the End User Agreement:

www.umlib.nl/taverne-license

Take down policy

If you believe that this document breaches copyright please contact us at:

repository@maastrichtuniversity.nl

providing details and we will investigate your claim.
}

Copyright and moral rights for the publications made accessible in the public portal are retained by the authors and/or other copyright owners and it is a condition of accessing publications that users recognise and abide by the legal requirements associated with these

- Users may download and print one copy of any publication from the public portal for the purpose of private study or research.

- You may not further distribute the material or use it for any profit-making activity or commercial gain

If the publication is distributed under the terms of Article $25 \mathrm{fa}$ of the Dutch Copyright Act, indicated by the "Taverne" license above, 


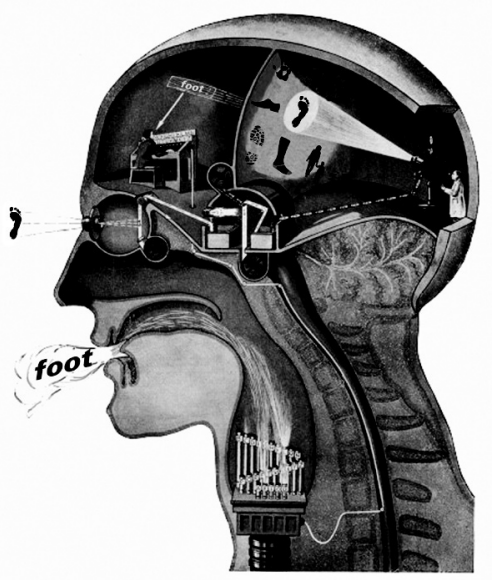

UNDER

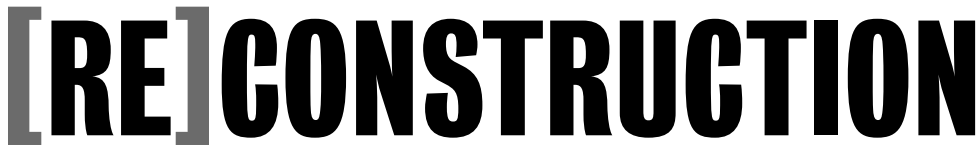

Neurocognitive correlates of pseudo-memories

MAARTEN J.V. PETERS 
The research presented in this dissertation was supported by the Netherlands Organization for Scientific Research (NWO, The Hague) grant number 452-02-006 awarded to Dr. M. Jelicic.

[design] KURT KNAEPEN EN MAARTEN PETERS

[cover image] KURT KNAEPEN - BILZEN

[print] DATAWYSE-UNIVERSITAIRE PERS MAASTRICHT

[ISBN] 978-90-5278-631-5

\section{(C) 2007 MAARTEN PETERS, MAASTRICHT}

All rights are reserved. No part of this book may be reproduced or transmitted in any form or by any means, without written permission from the author or, when appropriate, the publisher of the article. 


\section{PROEFSCHRIFT}

ter verkrijging van de graad van doctor aan de Universiteit Maastricht, op gezag van de Rector Magnificus, Prof. Mr. G.P.M.F. Mols volgens het besluit van het College van Decanen, in het openbaar te verdedigen op donderdag 7 juni 2007 om 12.00 uur

door

MAARTEN JEAN VALENTIN PETERS
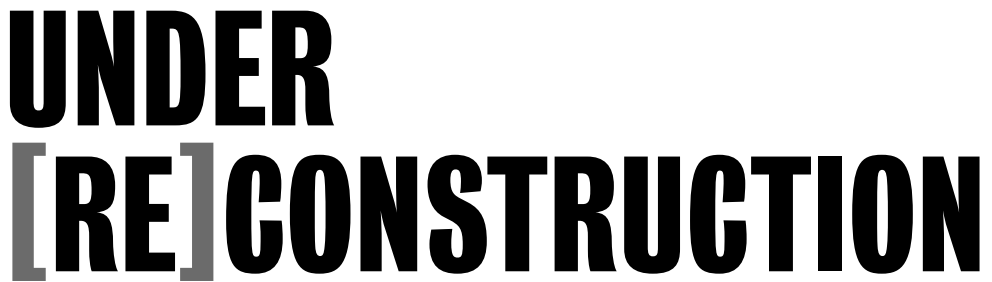

Neurocognitive correlates of pseudo-memories

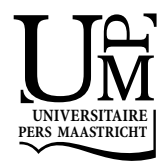


PROMOTOR:

PROF. DR. H.L.G.J. MERCKELBACH

CO-PROMOTOR:

DR. M. JELICIC

BEOORDELINGSCOMMISSIE:

PROF. DR. J. JOLLES (VOORZITTER)

PROF. DR. A.R. ARNTZ

PROF. DR. S. BRÉDART (UNIVERSITÉ DE LIÈGE)

PROF. DR. E.H.F. DE HAAN (UNIVERSITEIT UTRECHT)

DR. R.W.H.M. PONDS 

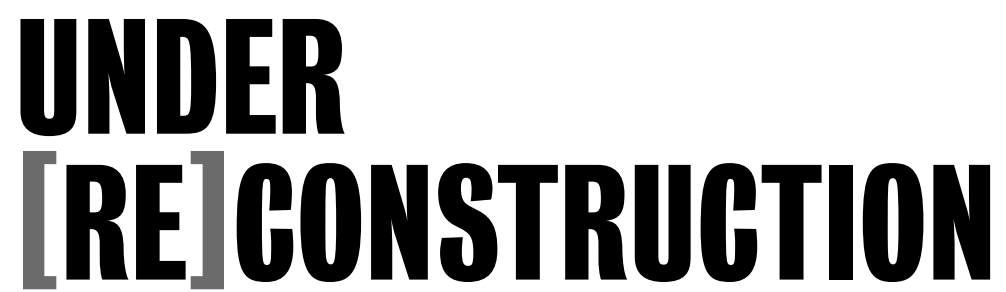

Neurocognitive correlates of pseudo-memories 


\section{GONTENTS}

\section{[1] General introduction}

Neuropsychology and pseudo-memories

Some typical cases: Case Bob Hunter \& Case JD 13

Memory metaphors: Reconstructing pieces of the memory puzzle $\quad 15$

Familliarity vs. recollection 16

$\begin{array}{ll}\text { Pseudo-memory paradigms } & 18\end{array}$

$\begin{array}{ll}\text { The neuropsychology of pseudo-memory } & 21\end{array}$

Brain damage and aging $\quad 22$

Functional Imaging data $\quad 24$

Clinical populations:The case of schizophrenia 25

Individual differences $\quad 26$

Executive functions $\quad 27$

Working memory $\quad 27$

Inhibition of stereotype activation $\quad 28$

$\begin{array}{ll}\text { Urgent questions } & 29\end{array}$

Outline of this thesis $\quad 30$

\section{PART [A] NORMATIVE DATA AND PSYCHOMETRIIGS}

[2] INDUGING PSEUDO-MEMORIES 35

A Dutch version of the Deese/Roediger-McDermott paradigm

$\begin{array}{ll}\text { Abstract } & 37\end{array}$

Introduction $\quad 38$

Method $\quad 40$

Results $\quad 42$

Discussion $\quad 47$

[3] THE RANDOM NUMBER GENERATION TASK 51

Psychometric properties and normative data of an executive function task in a mixed sample

Abstract 53

Introduction $\quad 54$

Study $1 \quad 56$

Study $2 \quad 57$

Study $3 \quad 59$

Study $4 \quad 61$

General discussion 63 


\section{PART [B] SOURCE MONITORING, PSEUDO-MEMORIES, AND MEUROCOGNITIVE FUNGTIONS IN UNDERGRADUATES}

\section{[4] RECOLLEGTING WORDS NEVER PRESENTED}

PART I

Mild executive dysfunctions in undergraduate students

Abstract

Introduction

72

Method

Results

Discussion

[5] RECOLLEGTING WORDS NEVER PRESENTED PART II

Poor working memory capacity in undergraduate students

Abstract

Introduction

Study 1

Method

Results and discussion

Study 2

Method

Results and discussion

General discussion

[6] ACTION SOURCE MONITORING, SGHIZOTYPAL 99 TRAITS, AND WORKING MEMORY

Abstract

Introduction

Method

Results

Discussion 


\section{GONTENTS}

[7] SUPPRESSION OF STEREOTYPES LEADS TO 113 FALSE RECOLLECTIONS

Influence of inhibition instruction on memory accuracy

Abstract

Introduction

Method

Results

Discussion

[8] MEMORY EFFIGIENGY, SOURGE ATTRIBUTIONS, 127 AND EXECUTIVE DYSFUNGTIONS IN SGHIZOPHRENIA

Abstract

Introduction

Method

Results

136

Discussion

[9] Remembering PRevious lives and SOURGe 147 MONITORING

Abstract

Introduction

Method

Results

154

Discussion 
Source monitoring in schizophrenia (-like symptomatology)

Encoding and consolidation

Retrieval

Future directions

Personality characteristics, neurocognitive functions, and pseudo-memories

Neurocognitive functions and pseudo-memory

Divided attention intermezzo

Social influence, pseudo-memories, and behavioural consequences

Cognitive neuroscience and pseudo-memories

Clinical implications

Forensic implications

The MP3 murder case

Neuropsychology in the judicial system

Final remarks

[NOTES]

[R]EFFERENGES 



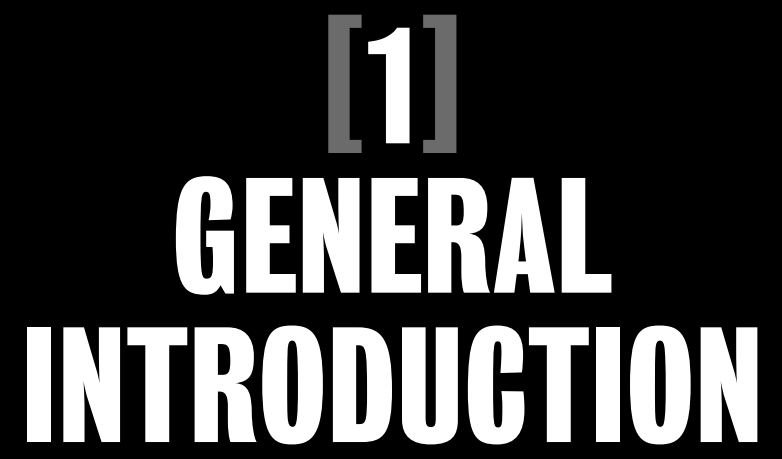

Neuropsychology and pseudo-memories 



\section{CHAPTER [1] GENERAL INTRODUGTION}

Neuropsychology and pseudo-memories

An adjusted version of this chapter is published as: Peters, M.J.V., Jelicic, M., \& Merckelbach, H. (2006). Neuropsychology and pseudo-memories. In J.R. Dupri (Ed.), Focus on neuropsychology research (pp. 163-184). Hauppage, NY: Nova Science Publishers.

\section{Some typical cases}

\section{Case Bob Hunter ${ }^{1}$}

On February $19^{\text {th }} 1985$, at 3 a.m., two policemen found a confused man in the red light district of Amsterdam. He was unable to respond to questions and comments. The man made an anxious impression, was disoriented in time, place, and person and found himself in a state of mutism. The police suspected that he might have been involved in a fight and suffered from brain injury. A CT scan was made at the hospital, but no neuroanatomical abnormalities were detected. From March $1^{\text {st }}$ until June $20^{\text {th }} 1985$, this man stayed in a psychiatric hospital. He still was unable to talk, but communicated with other patients and clinicians by writing in French. In his writings, he referred to himself as Bob Hunter. Neurological examination showed no abnormalities, but his performance on several neuropsychological tests suggested residual signs of brain damage. His main complaint was that he could not remember anything before February $19^{\text {th }} 1985$.

During the spring of 2002, we saw Bob Hunter, who still had no autobiographical memory for events that took place prior to February $19^{\text {th }} 1985$. We administered a neuropsychological test battery and collected psychophysiological data. We found his most recent Full Scale IQ to be 110 . His score on a long term verbal memory task was excellent, but he performed poorly on a working memory task (i.e., digit span task). His performance on the Trail Making Task, which is sensitive to frontal (i.e., executive) dysfunctions, was also very poor. He scored below average on memory for famous events up until 1985 (e.g., Mayes, Downes, McDonald, Rooke, Sagar, \& Meudell, 1994; Sanders \& Warrington, 1971). However, his memory for news facts from 1985 through 2002 was intact. Bob Hunter did not show any autonomic responsivity (heart rate, skin conductance) when presented with information pertaining to his past or present state (e.g., the name of his mother). During an interview, three further features became apparent: A paresis (i.e., partial paralysis) on the left side of his body, an indifferent attitude, and a tendency to confabulate.

The patient's working memory impairments, his deficits in executive functions, and his tendency to confabulate clearly indicate right prefrontal cortex damage (see also Weinstein, 1996; Kopelman, 1999). Bob Hunter's left sided paresis further supports this interpretation. His autonomic hyporesponsivity is consistent with damage to the right dorsolateral prefrontal cortex (Caltagirone, Zoccolotti, Originale, Daniele, \& Mammucari, 1989; Tranel \& Damasio, 1994; Zocollotti, Scabini, \& Violani, 1982). 
Unfortunately, these signs were disregarded by the psychiatrists who treated him. Encouraged by the police, Bob Hunter first underwent a series of hypnosis sessions and, following this, treatment with the "truth serum" pentobarbital. Questions were based on the assumption that Bob Hunter had a military background. That this was not without effect became evident when Bob Hunter began to uncover memories about his Canadian background and his work as a CIA agent. Although these memories were detailed and compelling, they turned out to be full-blown pseudomemories. By coincidence, the police was able to establish the real identity of Bob Hunter. He was born in a Paris suburb and had never been to Canada or the US. One day, in February 1985, he decided to visit Amsterdam and when he came there, he became involved in a fight, during which he was injured, thereby suffering brain damage.

\section{Case $J D^{2}$}

On February $20^{\text {th }} 2002$, the remains of a man were found on a local industrial zone, situated near the river Maas in a suburb of the city Liège. The local police and detective department tried to discover the identity of this person and the possible antecedents of the case. After a few months, the investigation reached a deadlock. Two years later, after new eyewitness accounts in a local television program and matching DNA examination, the remains were identified as belonging to $\mathrm{Mr} \mathrm{K}$.

Further investigations showed that Mr K disappeared on the evening of June $30^{\text {th }}$ 2001. On that summer evening, he went for a swim in the river Maas, situated at the border of his village near the local industrial zone. Mr K was accompanied by his close friend Mrs JD with whom he had a secret relationship.

In the period shortly after his disappearance, Mrs JD was questioned as a witness. She acknowledged that she had accompanied Mr K during his swim on that summer night, but stated that she left after a quarter of an hour, while Mr K was still in the water. This was the last time she had seen $\mathrm{Mr} \mathrm{K}$ alive.

In 2004, after identifying Mr K, the police reopened the case. Several persons possibly involved in this case were interrogated, among them Mrs JD. After several long-lasting interrogations in the period between May-June 2004, Mrs JD was identified as the single suspect in this case, based on several incriminating statements. She was first charged with taking the life of Mr K in the period between June 2001 and February 2002. Later on, the indictment was changed into "burying and/or, hiding and/or transporting the body of the victim in question."

However, in 2003 Mrs JD had experienced two cerebral vascular accidents, located in the right posterior (parietal) brain areas and a left lacunar infarction. As a consequence, she suffered severe brain damage in these and related frontal brain areas. Mrs JD neglected her left visual field, had problems with articulation (dysarthria), and showed marked impairments in visuo-spatial orientation, recognition of faces, and source monitoring. That is, Mrs JD was unable to identify whether an 
event had really happened to her or whether she had only imagined it. These observations were corroborated by neuropsychological testing. Mrs JD showed a global cognitive deterioration, as indicated by a score of 23/30 on the Mini Mental State Examination. Processing speed, attention, working memory, and executive control (e.g., Concept ShiftingTask, Digit Span task) were impaired. Furthermore, she showed deficits on visuo-spatial memory tasks (Rey complex figure task, Warrington face recognition task), motor abilities (dynamical aspects of Luria), and visuo-construction (line bisection task, line crossing task). She had pronounced difficulties with learning complex verbal information (immediate recall of complex story), and also showed an increased tendency to add non-existing information to her memory traces (i.e., confabulations; Dalla Barba Confabulation Questionnaire; Dalla Barba, 1993). Mrs JD also had an increased tendency to rely on external suggestions (Gudjonsson Suggestibility Scale; Gudjonsson, 1997).

Nevertheless, according to the police and the prosecutor, the statements made by Mrs JD were accurate, and thus revealed intimate knowledge of the assassination of Mr K.

We cannot rely on the statements of Bob Hunter and JD as accurate reconstructions of their past experiences. Both Bob Hunter and JD suffered brain damage. To determine whether this damage affected their competence to give official accounts, one first has to know which brain structures and functions are involved in the reconstruction of memory. More importantly, knowledge is needed about the consequences of lesions to these brain areas for memory accuracy. In recent years, a vast amount of case studies and laboratory experiments in clinical populations have shown that (pre)frontal lobe damage gives rise to remembering information never presented (i.e., confabulations). Thus, the (pre)frontal cortex can be considered a key player in reconstructing our memories. This is nicely illustrated in the cases Bob Hunter and JD, where damage, either directly or indirectly via disconnecting neural networks, led to confabulations - one particular type of pseudo-memories.

What more can neuropsychology tell us about the reconstruction of memory and the creation of pseudo-memories? In this Chapter, we will give an overview of past, present, and future developments in neuropsychological research on pseudo-memories, thereby linking neurocognitive functions to pseudo-memories. The review below will serve as a starting point for the outline of this dissertation.

\section{Memory metaphors: Reconstructing pieces of the memory puzzle}

Throughout history, memory has been described in terms of different metaphors: From a wax tablet (Plato) up to the popular computer metaphor in our times (Draaisma, 2000; Merckelbach \& Wessel, 1998). However, apart from making analogies with saving, deleting, number of bytes, and so on, memory is more than just a matter of remembering and forgetting past events (e.g., Kopelman, 2002; Schacter, 1999). That is, 
events and experiences may be remembered in a distorted way. An eyewitness may, for example, remember a yellow taxi, when in fact the taxi was blue. These subtle changes in details of a memory have been termed distortions (Gudjonsson \& Clare, 1995). Sometimes events are completely fabricated, as was the case with Bob Hunter and JD. Even neuropsychological intact people may make such pseudo-memories (i.e., commission errors). An example is the eyewitness who remembers a gun, when in fact there was no gun at all. Throughout this dissertation, we will refer to this type of error as pseudo-memory although we are aware of the fact that the term "false memory" is more common. Regardless of terminology, an important point about distortions and pseudo-memories is that they make plain that the idea of memory as a reproductive entity, in which events are reproduced with photographical precision, is incorrect. Instead, memory is reconstructive, precisely because events are encoded in an incomplete and fragmentized way. When retrieving an experience, different fragments have to be combined to form an entity. Almost 40 years ago, Neisser used the analogy of the palaeontologist when describing memory. He wrote: "Out of the few stored bone chips we remember a dinosaur" (Neisser, 1967, p. 285).

Until recently, neuropsychological research in this domain was limited to case studies of brain damage patients with peculiar memory illusions (e.g., DelbecqDerousne, Beauvois, \& Shallice, 1990; Parkin, Bindschaedler, Harsent, \& Metzler, 1996; Schacter, Curran, Gallucio, Milberg, \& Bates, 1996a). The patients described in these case studies had a tendency to falsely recognize non-presented stimuli as being previously presented (e.g., Parkin et al., 1996), misattributed the source of their memories (e.g., Janowsky, Shimamura, \& Squire, 1989), or recollected fictitious events (e.g., Dalla Barba, 1993). It was in such case studies that the reconstructive nature of memory became most compelling. In recent years, inspired by ongoing debates about the "accuracy" of human memory and its legal implications (e.g., Koriat, Goldsmith, \& Pansky, 2000; Loftus, 2003), there has been an increased interest in neurocognitive research on pseudo-memories (e.g., Kopelman, 2002; Schacter, 1999, 2001). The introduction of modern imaging techniques such as functional Magnetic Resonance Imaging (fMRI) and Positron Emission Tomography (PET) has also stimulated new studies in this field (e.g., Kopelman, 1999, 2002; Schacter \& Slotnick, 2004). Before describing past and current findings of neuropsychological studies on pseudo-memories, we will first give a brief theoretical and methodological overview of pseudo-memories.

\section{Familiarity vs. recollection}

During the past century, a plethora of research has focused on how pseudo-memories can be explained. The early works of Burnham (1889) and Bartlett (1932) show that incorrect details of an event can be implanted and that memory reconstruction is partly based upon general schematic knowledge (cognitive schemas). Since then, several studies have documented similar findings, converging on the notion that 
proactive interference (i.e., memories can be distorted by earlier experienced events) or retroactive interference (memories can be distorted by experiences that are experienced later on) produce such pseudo-memories. At this moment, cognitive theories on memory reconstruction generally focus on the trade-off between two competing attributions: Familiarity and recollection (e.g., Brainerd \& Reyna, 2001; Jacoby, 1991; Jacoby, Kelley, Brown, \& Jasechko, 1989, Johnson, Hashtroudi, \& Lindsay, 1993; Schacter, 1999), thereby also incorporating findings from cognitive neuroscience on memory distortions and pseudo-memories like the Constructive Memory Framework proposed by Schacter, Norman, and Koutstaal (1998; Dodson \& Schacter, 2002a).

Familiarity can be described as a global impression of events. It refers to merely "knowing" that an event took place (Tulving, 1985) i.e., remembering the general gist of experiences that is consistent with many possible events (e.g., "I remember that I took a taxi somewhere this week"). Recollection, on the other hand, is accompanied by impressions of item-specific knowledge and thus gives access to representations of detailed perceptual and contextual features (e.g., "I clearly remember the taxi picking me up an 12.30 p.m. on Saturday, that it was black, that the taxi driver was Hispanic, and that I had to pay 15 euros"). In terms of the "Remember-Knowing" distinction proposed by Tulving (1985), recollection can be described as "remembering" an event. How then can this trade-off between two competing attributions lead to memory distortions and pseudo-memories?

New experiences are organized in patterns of features that represent different aspects of the experience (Dodson \& Schacter, 2002a; Schacter et al., 1998), like perceptual details (black taxi, Hispanic), contextual information (NewYork, Saturday), and so on: "The things I did on my birthday, the things I ate on that day, the various presents I got, the place where I celebrated my birthday." During encoding and consolidation, these perceptual and contextual features need to be bound together to form a "coherent" memory representation (i.e., the feature binding). It is also necessary to keep these bound representations separated from each other (pattern separation), thereby decreasing the amount of overlap between memory representations. The retrieval of this information requires an act of pattern completion, whereby the features belonging to a past experience are activated and the activation spreads out over other features (spreading activation). This phase of retrieval also involves postretrieval monitoring (i.e., criterion setting), in which it has to be determined whether the memory representation is a veridical recollection of an experienced event or a fantasy. This critical step in retrieval is also known as source attribution. Technically speaking, strict criterion setting promotes correct source attributions. When memory representations have to be recalled or recognized, a reliable and valid heuristic is to strictly rely on perceptual and contextual information surrounding the memory representation and to base one's source attribution judgments on the vividness (i.e., richness of perceptual and contextual details) of the memory representation in order to obtain an accurate recollection (Roediger, Watson, McDermott, \& Gallo, 2001). 
Problems in the reconstruction of memory representations arise when encoding, consolidation, or retrieval function are suboptimal. More specifically, when feature binding, pattern separation, pattern completion, or criterion-setting fall short (either individually or in combination), memory errors occur. Factors that can affect feature binding, pattern separation, pattern completion, or criterion setting are individual differences in personally relevant factors (e.g., suggestibility, brain damage, subjective plausibility, cognitive functioning; internal factors) and environmental factors (e.g., social influence; external factors). For example, when one is confronted with a statement of another eyewitness in a robbery case, this environmental factor will increase the event's familiarity. And this will a fortiori to be the case, if one scores high on suggestibility, which is a personally relevant factor.

Distinctive perceptual and contextual details need to be encoded and consolidated efficiently in order to retrieve an accurate recollection of this event. However, when due to internal or external factors, encoding or criterion setting during retrieval is inefficient, one will systematically retrieve the general theme and may take this familiarity feeling for granted. People then may misattribute the item's familiarity to a variety of extraneous sources, such as thinking that you saw a particular person robbing a bank, when in fact you rely on a statement made by another witness. Thus, once an event is perceived as plausible (thereby increasing familiarity), people may come to believe that the event has happened to them (belief). The following step would be the acceptance of these beliefs as genuine memories (i.e., true recollections), which requires loose criterion setting and, as a result, a source misattribution (e.g., Hart \& Schooler, 2006; Pezdek, Finger, \& Hodge, 1997; Smeets, Merckelbach, Horselenberg, \& Jelicic, 2005).

The strategy of relying on recollection in order to avoid familiarity-based misattributions and pseudo-memories is supported by a variety of empirical evidence. Studies on criterion setting and source misattribution are often referred to as source monitoring research (Johnson et al., 1993). Source monitoring is closely related to criterion setting. It is a mechanism that serves as a screening and controlling device, involved in determining the source of information at retrieval and includes three different abilities: the ability to differentiate between one's own verbal or motor actions and those imagined (internal source monitoring/self-monitoring), the ability to distinguish between information from two external sources (external source monitoring), and the ability to distinguish between self-generated and external verbal or motor actions (reality monitoring). Recent neurocognitive findings localize these abilities in a network encompassing primarily the prefrontal cortex (e.g., Johnson et al., 1993).

\section{Pseudo-memory paradigms}

During the nineties, Sir Frederic Bartlett's groundbreaking work (1932) on the reconstructive aspects of memory resurfaced, inspired by the debate about the accuracy of traumatic memories recovered during psychotherapy (Loftus, 1993; Loftus \& Ket- 
cham, 1994; Read \& Lindsay, 1997). Catalyzed by this revival, cognitive psychologists developed various experimental paradigms to elicit memory errors (e.g., Loftus, Miller, \& Burns, 1978). Paradigms can be classified as memory distortion paradigms, in which accurate memory traces are distorted by misinformation, and pseudo-memory paradigms in which the misremembered information results from inferences or constructions that a person creates internally and then later mistakes for events that really happened. We now briefly describe the most common techniques.

Exposure to (post-hoc) misinformation can have detrimental effects on the accurate recollection of a specific event (for a review, see Loftus, 2005; Loftus, Feldman \& Dashiell, 1995). An example may clarify this: Suppose that $X$ is a witness to an accident, caused by a car that did not stop at a stop sign. During the interrogation of $X$, a police officer asks $X$ what happened when the car arrived at the yield sign. Research by Loftus and Palmer (1974) and Loftus and colleagues (1978) shows that there is a serious possibility that $X$ will misremember that the car did not stop at the yield sign. Thus, subtle suggestions provided after the event has occurred (i.e., post-hoc) may distort the way in which people come to remember the event (see also Ceci \& Bruck, 1993; Crombag, Wagenaar \& van Koppen, 1996; Jelicic, Smeets, Peters, Candel, Horselenberg, \& Merckelbach, 2006;Ost, Vrij, Costall \& Bull, 2002; Smeets, Jelicic, Peters, Candel, Horselenberg, \& Merckelbach, 2006).

Encouraging people to fantasize about events that they never experienced is the crux of the imagination-inflation paradigm. Imagining a fictitious event can lead to an increase in subjective confidence that the event did take place (Garry, Manning, Loftus, \& Sherman, 1996). Consider the following example: A young man is asked to imagine how he, at age 5, was a passenger in a hot air balloon. According to the man's parents, such an event never took place. After several days, he is asked to assign a confidence rating to the following item: "At age 5, I flew as a passenger in a hot air balloon". It is likely that he will overestimate the probability of the balloon trip compared to items that were not imagined (Garry et al., 1996; see also Horselenberg, Merckelbach, Muris, Rassin, Sijsenaar, \& Spaan, 2000).

"Did I do this, did I only imagine doing this, or was this done by someone else?" are the most important questions participants have to ask themselves during a traditional source monitoring paradigm (e.g., Anderson, 1984; Johnson et al., 1993; Parks, 1997). Thus, rather than only making old-new distinctions, in the source monitoring paradigm, people are asked to remember and report the source of the memory item. To give an example: A card depicting questions is shown to a participant for a period of $5 \mathrm{sec}$, followed by a blank card for $2 \mathrm{sec}$. The participant is instructed to read each question on the card in silence and to be prepared to say the answer out loud without actually verbalizing unless he/she is specifically told to do so. After this card, he/she receives the instruction "Say out loud" or the next trial giving the following question in the series is given. After the study phase, a forced choice recognition task is presented to the participant, consisting of the original questions, each being paired 
with new questions with similar content. For both types of questions, the participant has to indicate whether they are "old" or "new" and when the participant says "old", he/she has to specify whether he/she verbalized or only imagined the answer to the question (i.e., source monitoring).

In semantic relatedness paradigms (e.g., Deese, 1959b; Roediger \& McDermott, 1995), participants are exposed to cues referring to a critical item that is never presented. In such paradigms, pseudo-memories are not explicitly established by an outside source, but are created by a cascade of internal processes. For example, in the Deese/Roediger-McDermott (DRM) paradigm, initially developed by Deese (1959b) and later modified by Roediger and McDermott (1995), people are asked to remember related words, such as bed, nap, pillow, and snooze, all of which are associated to a common word, in this particular example, the word sleep. The word sleep, however, is not presented in the study list and serves as a critical lure at test. Following each list presentation, participants are asked to recall the studied words. Once all lists have been presented and recalled, participants are typically given a recognition test comprising of the studied words, unrelated lures, and critical lures. Roediger and McDermott (1995) reported that on average, participants falsely recognized 65-80 per cent of the non-presented critical lure words. These findings were replicated in a follow-up study by Stadler, Roediger, and McDermott (1999).

In essence, the methodology of the pseudo-memory implantation paradigms is strongly related to the (post-hoc) misinformation paradigm. Yet, they differ in one important respect: Instead of distorting memory traces by post-hoc misinformation (e.g., a blue taxi, while in fact the taxi was white), planting memories for an entirely new event that was never experienced is the crux of the implantation paradigms (e.g., Loftus \& Pickrell, 1995). In a standard implantation paradigm, participants are given brief descriptions of events that supposedly occurred in the past while participants were accompanied by a close relative. Most of the events are true events (corroborated by the relative), but one event is fictitious (e.g., being lost in a shopping mall). Participants are then asked to try to write about these events in detail. Later, they are interviewed about the events. Loftus and Pickrell (1995) found that a quarter of the participants in their study were "remembering" the fictitious event of being lost in the shopping mall.

What post-hoc misinformation, imagination-inflation, source monitoring, semantic-relatedness techniques, and pseudo-memory implantation all have in common is that they produce source monitoring problems (e.g., Johnson et al., 1993). That is, such techniques put increased pressure on feature binding, pattern separation, pattern completion, and/or criterion setting. When these encoding and retrieval functions are suboptimal, memory distortions or even pseudo-memories may arise. In that case, participants take familiarity feelings for granted, thereby misattributing the item's familiarity to a variety of extraneous sources, such as believing that a certain event must have happened. Obviously, the tendency to accept plausible, 
internally generated beliefs as authentic events is an important step in the direction of a full-blown pseudo-memory (see also, Smeets et al., 2005). We now turn to the contribution of neuropsychology to pseudo-memories.

\section{The neuropsychology of pseudo-memory}

Before linking neuropsychology to pseudo-memories, we first start with a brief review on the neuropsychology of declarative memory (episodic, semantic, spatial). Scholarly reviews on this topic can be found in Fletcher and Henson (2001), Greenberg and Rubin (2003), and Squire and Schacter (2002; see also Kopelman, 2002; Moscovitch et al., 2005). ${ }^{3}$ Briefly, these authors argue that when attention is paid to stimuli, different regions of the primary sensory and association cortex are activated. Thus, a visual stimulus activates the visual cortex, whereas auditory stimulation activates the auditory cortex. The role of the medial temporal lobe (hippocampal complex) and related structures in the diencephalon (MTL/D) can best be regarded as a switchboard, linking different brain regions that are simultaneously activated during the encoding of a specific event. The hippocampus has a high number of reciprocal neural connections, potentially mediating transfer of information from the hippocampal complex to the neocortex. Therefore, this brain structure may be seen as an intermediate linking site between experienced events and their representations in the neocortex. When one wants to recollect/retrieve specific events, certain structures in the medial temporal lobe mobilize different regions in the sensory and association cortex, thereby creating feature binding and pattern separation.

The prefrontal cortex is involved in search strategies and the evaluation of their results. Its primary role is evaluating and monitoring relevant information and inhibiting irrelevant information during pattern completion and criterion setting. The aim of this post-retrieval control is to verify the appropriateness of the retrieved information in a given context (error-checking; Koriat \& Goldsmith, 1996). That is, the prefrontal cortex organizes, selects, and activates the correct representation from the various representations that are encoded and consolidated at different points in time (Nyberg, Cabeza, \& Tulving, 1996). More specifically, the left prefrontal cortex organizes the encoded information in the most efficient way for later remembering (Fletcher, Shallice, Frith, Frackowiak, \& Dolan, 1998). In contrast, the right prefrontal cortex guides retrieval (Kapur, Craik, Jones, Brown, Houle, \& Tulving, 1995; Wheeler, Stuss, \& Tulving, 1997) and some authors (e.g., Markowitsch, 1996) believe that it is particularly involved in the retrieval of autobiographical memory.

In his review article on the neuropsychology of pseudo-memories, Parkin (1997) states that prefrontal damage may lead to different memory distortions. Patients with prefrontal damage show a decreased usage of post-retrieval control (e.g., monitoring, evaluation, inhibition, criterion setting). This often leads to increased reliance on familiarity based processes and, thus, endorsement of irrelevant memory representations during the retrieval of a specific event (Shimamura, 1995). 
According to the Constructive Memory Framework by Schacter and colleagues (1998; Dodson \& Schacter, 2002a), memory distortions and pseudo-memories are modulated by neuropsychological factors operating primarily at encoding or retrieval stages of memory. As described above, during encoding perceptual and contextual features need to be bound together to form accurate representations. However, these representations also need to be distinctive. During retrieval, specific criteria need to be employed to be certain that the retrieved representation is the correct event rather than the product of a dream or fantasy. Lax criterion setting can lead to source misattributions and later memory distortion or pseudo-memories.

When combining the insights of what we know about the neuropsychology of memory, it seems clear that the medial temporal lobes are responsible for encoding and retrieval of recent experiences, while the prefrontal cortex controls the retrieval of old experiences. More specifically, the prefrontal cortex is involved in retrieval focus, criterion setting, updating, inhibition/suppression, and monitoring of information. Plainly, both require activation and manipulation of distinctive information during encoding, and cognitive inhibition and active manipulation/monitoring of schema-related material during retrieval. Evidence for the distinct roles of temporal and frontal areas in memory can be found in case studies and empirical studies involving patients with focal brain damage, aging research (including dementia of the Alzheimer type), research in clinical populations, research using neuroimaging techniques, and individual difference studies.

\section{Brain damage and aging}

As mentioned before, initial evidence for the role of the prefrontal cortex in the creation of pseudo-memories came from several case studies (e.g., Delbecq-Derousne et al., 1990; Parkin et al., 1996; Schacter et al., 1996a). The patients described in these papers had the initials JB (Parkin, Leng, Stanhope, \& Smith, 1988; Parkin et al., 1996), BG (Curran, Schacter, Norman, \& Gallucio, 1997; Schacter et al., 1996a), and RW (Delbecq-Derousne et al., 1990; see also patient MR; Ward, Parkin, Powell, Squires, Townshend, \& Bradley, 1999; patient JT; Young, Flude, Hay, \& Ellis, 1993; patients WJ and BH; Rapcsak, Polster, Comer, \& Rubens, 1994). Patients JB and RW were both diagnosed with a ruptured aneurysm of the anterior communicating artery (ACoA), whereas patient BG was diagnosed with an infarction located in the right frontal lobe. These three patients exhibited an extremely high rate of false recognition errors in a forced-choice recognition task. The data of JB further revealed a recall impairment in which intrusion rates (i.e., producing non-presented words) were abnormally high. Ward and colleagues (1999) described a patient (MR) who was able to correctly recognize famous people, but also had a strong tendency to falsely classify unfamiliar people as familiar (see also Rapcsak, Reminger, Glisky, Kaszniak, \& Comer, 1999). This patient had had a lacunar infarction in the area above the left lateral ventricle adjacent to the left frontal horn. 
Recent attempts to explore the separate contributions of medial temporal and prefrontal areas to memory reconstruction have supported the findings from these case studies. A study by Melo, Winocur, and Moscovitch (1999) examined patients with either isolated damage to the medial temporal (MTL/D amnesics) or the prefrontal areas (FL nonamnesics) or a combination of both (medial temporal and prefrontal; FL amnesics), and healthy controls using the DRM paradigm. At recall, FL amnesic patients showed a lower proportion of critical lure intrusions than did controls. However, MTL/D amnesic patients and FL nonamnesic patients made more critical lure intrusions than control participants. At recognition, both MTL/D amnesics and FL amnesics made significantly less critical lure intrusions in comparison to FL nonamnesics. In conclusion, Melo and colleagues (1999, p. 356) state: "The tendency towards exaggerated levels of false recall and recognition by frontal lobe patients may result from defective monitoring that allows them to generate only a vague or unfocused representation of characteristic features of the target words against which test items are matched." In contrast to this, amnesic patients with MTL/D lesions show markedly reduced levels of false recognition which has been taken as evidence for impairments in the encoding, maintenance, and retrieval of recollections and the accompanied feeling of familiarity (Schacter, Verfaellie, \& Koutstaal, 2002; Verfaellie, Page, Orlando, \& Schacter, 2005).

Relative to younger adults, neurologically intact elderly tend to make more false recognition errors. Using the DRM paradigm, a study by Butler, McDaniel, Dornburg, Roediger, and Price (2004) investigated the relationship between prefrontal lobe functioning and age differences in false recall. These authors found that older adults were less successful in reproducing studied items and more often falsely recalled the non-presented critical lure words in comparison to younger adults, which is in line with other studies (Balota, Cortese, Duchek, Adams, et al., 1999; Budson, Sullivan, Daffner, \& Schacter, 2003; Cohen \& Faulkner, 1989; Dywan \& Jacoby, 1990; IntonsPeterson, Rocchi, West, McLellan, \& Hackney, 1999; Koutstaal \& Schacter, 1997; Norman \& Schacter, 1997; see review by Schacter, Koutstaal, \& Norman, 1997). These pseudo-memories were not related to the length or elaboration of the learning phase (Kensinger \& Schacter, 1999). Most importantly, Butler and colleagues (2004) showed that these pseudo-memories were intimately linked to measures of prefrontal lobe functioning (i.e., executive functions). That is, only older adults characterized by poor prefrontal lobe functioning exhibited heightened levels of false recall. Older adults with intact frontal lobe functioning and young adults had similar levels of accurate and false recall. In line with this, imaging studies of older adults show deviant activation patterns in the prefrontal cortex during memory tasks. This is most prominent when they have to rely on controlled retrieval strategies during these tasks (Schacter, Savage, Alpert, Rauch, \& Albert, 1996b).

Recent studies have begun to explore pseudo-memories in individuals who suffer from Dementia of the Alzheimer Type (DAT; see Balota et al., 1999; Budson et 
al., 2003; Budson,Todman, \& Schacter, 2006; Marsch, Balota, \& Roediger, 2005; Mitchell, Sullivan, Schacter, \& Budson, 2006; Pierce, Sullivan, Schacter, \& Budson, 2005; Waldie \& Kwong See, 2003; Watson, Balota, \& Sergent-Marshall, 2001). Basically, these studies show that DAT patients are especially susceptible to pseudo-memories when pre-existing semantic information is activated. Budson, Daffner, Desikan, and Schacter (2000) tested DAT patients using the Deese/Roediger-McDermott paradigm (Deese, 1959b; Roediger \& McDermott, 1995). Surprisingly, these researchers found that, whereas DAT patients were significantly less susceptible than controls to falsely recognize the critical lure word after a single presentation of the study lists, this pattern reversed when study lists were presented five times.

The deficits in recall and recognition in DAT are related to their MTL/D dysfunction and mild prefrontal lobe dysfunctions (e.g., Dalla Barba, Nedjam, \& Dubois, 1999). As long as stimuli are presented incidentally, it seems that DAT patients cannot rely on familiarity or recollection for making source attributions. However, after repeated presentations, familiarity with the presented semantic related items increases. Due to mild prefrontal lobe dysfunctions, the error-checking cannot inhibit these familiarity feelings evoked by lure items. Lack of efficiency in prefrontal mediated inhibitory control and post-retrieval monitoring system could increase the amount of familiarity based source misattributions.

\section{Functional Imaging data}

Paralleling the increased interest in neuroimaging during the last decades, pseudomemory neuroimaging studies have accumulated. A complete description of the various $\mathrm{fMRI}, \mathrm{PET}$, and electroencephalogram (EEG) studies is beyond the scope of this Chapter (for a thorough overview of research in this area see Schacter \& Slotnick, 2004). Below, we will highlight one prototypical study.

In a PET study, Schacter, Reiman, Curran, Yun, et al. (1996c) focussed on true and false recognition in the DRM task. They found that an increased activation in left temporal region was found during accurate recall of presented words. Moreover, there appeared to be an increase in right prefrontal activity during false recognition of critical lures. Using fMRI during a follow-up study, the same researchers noted a similar pattern (Schacter, Buckner, Koutstaal, Dale, \& Rosen, 1997). In this study, a delayed onset of prefrontal activation during false recognition occurred, a phenomenon that the authors relate to suboptimal controlling of the prefrontal cortex during retrieval.

A number of other studies have tried to disentangle the brain mechanisms involved in accurate and pseudo-memories (e.g., Rodriguez-Fornells, Kofidis, \& Münte, 2004; for reviews, see Dodson \& Schacter, 2002a; Gonsalves \& Paller, 2002; Schacter \& Slotnick, 2004). Of these studies, different neuroimaging studies have pointed to the right dorsolateral prefrontal cortex as supporting this error-checking/ post-retrieval monitoring (e.g., Allan, Dolan, Fletcher, \& Rugg, 2000; Henson, Shallice, \& Dolan, 
1999). By and large, neuroimaging research demonstrates that, in addition to a (modest) involvement of the medial temporal lobe, the prefrontal cortex plays a leading role in error-detection, notably pseudo-memories during retrieval (see also Mitchell, Dodson, \& Schacter, 2005).

\section{Clinical populations: The case of schizophrenia}

Studies on the antecedents of pseudo-memories are not limited to neurological patients, aging research, and the cognitive neuroscience field. In recent years, a plethora of research has investigated source misattributions and pseudo-memories in schizophrenia (e.g., Brébion, Gorman, Malaspina, \& Amador, 2005; Henquet, Krabbendam, Dautzenberg, Jolles, \& Merckelbach, 2005; Moritz, Woodward, Cuttler, Whitman, \& Watson, 2004; Nienow \& Docherty, 2004; Waters, Maybery, Badcock, \& Michie, 2004).

Memory performance of schizophrenic patients is characterized by leaving out presented information (i.e., omission errors). That is, schizophrenic patients have a tendency to omit information when asked to recall or to recognize specific verbal or non-verbal material (e.g., Moritz et al., 2004; see Aleman, Hijman, de Haan, \& Kahn, 1999 , for a meta-analysis). However, source monitoring mechanism is also markedly impaired in schizophrenia (Johnson et al., 1993). Schizophrenic patients consistently show a deficiency in internal source attributions (e.g., "Did I do this or did I only imagine this?"; Nienow \& Docherty, 2004) and reality monitoring (e.g., "Did I say this or did someone else say this to me?"; Brébion, Gorman, Amador, Malaspina, \& Sharif, 2002; Moritz, Woodward, \& Ruff, 2003). A recent study by Waters et al. (2004) found that deficits in binding contextual cues together might explain commonality of these source misattributions in schizophrenia. Forty-three schizophrenic patients and 24 healthy controls were subjected to a paradigm in which item memory could be assessed in conjunction with memory for both source and temporal information. In this paradigm, 48 common household objects were presented to the participants. Half were allocated to the "watch" condition, in which the participants watched the experimenter pair these household objects. The other half of the objects were allocated to the "perform" condition, in which participants performed the pairings themselves. In two sessions, a series of cards informed the participant to position objects next to one another or to watch the experimenter perform the action. Next, participants underwent a recognition task consisting of 24 pairs of objects. Of these, 16 pairs were kept in their original combination, while 8 pairs were recombined into new pairs. Participants had to indicate whether each pair was an original or a new pair. For intact pairs, they also had to specify source (self/experimenter) and temporal (session 1 or 2) context. It was found that schizophrenic patients were less accurate in identifying the source and temporal context and were unable to combine contextual cues together to form an integrated representation of the event, which indicates a specific feature binding problem. 
As described above, binding of contextual cues depends on encoding and the ability to "reactivate" the correct information during retrieval (i.e., post-retrieval monitoring). As the memory deficits observed in schizophrenia closely parallel those of patients with MTL/D damage, one could argue that the underlying mechanisms are similar (i.e., inefficient feature binding during encoding). Alternatively, prefrontal lobe dysfunctions may also underlie memory deficits in schizophrenia. After all, the prefrontal lobes play an important role in monitoring the retrieval of encoded events in memory. Until now, the role of executive dysfunction in explaining source misattributions and pseudo-memories in schizophrenia has not been systematically studied (but see Moritz et al., 2003).

\section{Individual differences}

Clearly, some individuals are more susceptible to source misattributions and pseudo-memories than others. As noted above, there are neurocognitive precursors for source misattribution and pseudo-memories. Indeed, recent aging and lesion studies suggest that executive functions such as post-retrieval monitoring and inhibition play a critical role in the creation of source misattributions and pseudo-memories

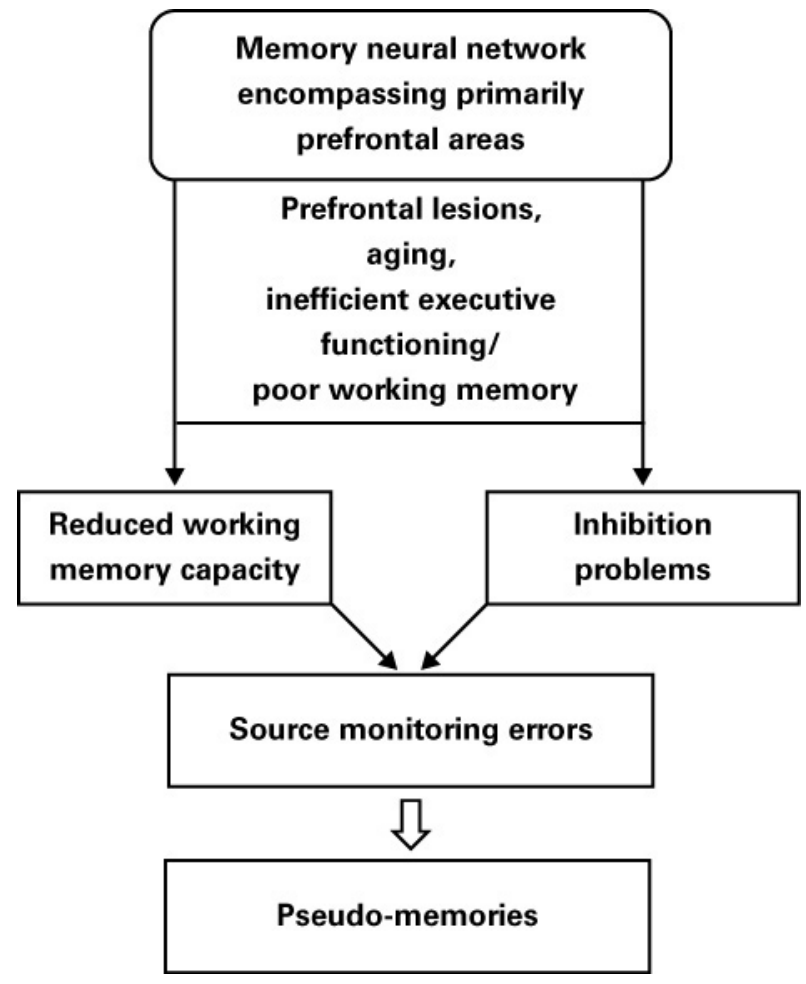

FIGURE 1.1: Model OF THE INVOLVEMENT OF PREFRONTAL AREAS IN PSEUDO-MEMORIES. 
(SEE FIGURE 1.1). It would not be too farfetched to assume that even in young, healthy people, individual differences in specific neurocognitive functions might be related to source monitoring and pseudo-memories. Recent individual difference studies provide some tentative evidence that this could be the case for subtle executive dysfunctions, working memory, and inhibition of schematic knowledge.

\section{Executive functions}

A fine example of the possible link between subtle neurocognitive dysfunctions and pseudo-memories is an individual difference study by Alexander, Goodman, Schaaf, Edelstein, Quas, and Shaver (2002). These authors interviewed children ( $n=51)$ between the ages of 3 and 7 years about an inoculation that they had undergone two weeks earlier. Children's memory accuracy and suggestibility were examined in relation to their stress levels during inoculation, parental attachment styles, and cognitive inhibition (i.e., ability to suppress irrelevant information). The authors anticipated that children with higher levels of cognitive inhibition would provide more accurate information and would be less suggestible than children with lower levels of inhibition. In support of this, children with poor cognitive inhibition exhibited more pseudo-memories on a free recall task about inoculation and more often accepted misleading information than children with adequate inhibition capacity. This difference remained, even when controlling for age. More recently, Lödvén (2003) investigated the underlying mechanisms of age effects on pseudo-memories. A total of 146 participants, aged 20 to 80 years were subjected to tasks measuring processing speed, inhibition, episodic memory performance, and pseudo-memories. Results revealed that levels of pseudo-memories increased with age. Using structural equation modeling, Lödvén found that impaired inhibitory control affected susceptibility to pseudo-memories indirectly via episodic memory performance. That is, participants with impaired inhibitory control scored low on episodic memory tasks. Moreover, participants scoring low on episodic memory tasks more often falsely recalled the critical lure in a DRM paradigm, compared to participants scoring high on these episodic memory tasks.

\section{Working memory}

Working memory is closely related to prefrontal cortex mediated executive functions. A paper by Reinitz and Hannigan (2004) related pseudo-memories to working memory capacity. In one of their three experiments, the authors had participants ( $n$ $=48$ ) in an experimental group study pairs of compound words (e.g., toothpaste, headache) while simultaneously monitoring specific digits (digit monitoring task), such that at the end they had to report the total number of even digits. The authors found that participants in the digit monitoring condition more often tended to falsely remember never presented compound words (e.g., toothache) than control participants. The results indicated that intact working memory is necessary for binding 
stimulus parts together in episodic memory. Inefficient working memory leads to binding failures, and subsequently to an increase in pseudo-memories for never presented compound words.

McCabe and Smith (2002) examined age differences in the ability to suppress pseudo-memories, as elicited by the DRM paradigm. In two separate experiments, one group of younger and older adults were subjected to a standard DRM procedure. Other young and older adults were explicitly warned before studying the DRM lists that the presented words all relate to a semantically non-presented word. Still other young and older adults were warned after studying but before testing. Lists were presented at different rates ( $4 \mathrm{sec} /$ word or $2 \mathrm{sec} /$ word) to each of the groups. Individual difference measures like working memory were also administered. Young adults were better in discriminating between studied words and critical lure words when warned about the DRM effect (either before or after the study phase). Older adults were able to discriminate between studied items and critical lure words when given warnings before the study phase, but not when given warnings after the study phase and before retrieval. As to the individual difference measures, working memory capacity predicted false recognition following study and retrieval warnings. That is, reduced working memory capacity was associated with higher rates of pseudomemories (see also Watson, Bunting, Poole, \& Conway, 2005). McCabe and Smith (2002) concluded that discriminating between similar sources of activation critically depends on working memory capacity, which declines with advancing age. This leads to a heightened susceptibility to pseudo-memories.

\section{Inhibition of stereotype activation}

Social psychology research has recently begun to address the relationship between prefrontal lobe functions and abstract schematic knowledge, like stereotype activation (Macrae, Bodenhausen, Schloerscheidt, \& Milne, 1999; Mitchell, Cloutier, Banaji, \& Macrae, 2006; Payne, 2005; Wood, Romero, Knutson, \& Grafman, 2005). A study by Mather, Johnson, and De Leonardis (1999) showed that relative to young participants, older participants with prefrontal lobe dysfunctions showed and increased tendency to rely on stereotypes when attempting to remember who said specific statements in an earlier presented video (see also Von Hippel, Silver, \& Lynch, 2000).

A stereotype can be defined as a widely held schematic belief about a certain social group (Graham \& Lowery, 2004; Hamilton \& Sherman, 1994). Concerning stereotype activation, an ambiguity is seen when it comes to its influence on memory. Stereotypes facilitate encoding and retrieval of stereotype-consistent information, but they may also lead people to remember stereotype-consistent details that were not present (e.g., Araya, Ekehammar, \& Akrami, 2003). False stereotype-consistent information is especially likely to be reported when the cognitive inhibition function is overloaded, i.e., when people are instructed to deliberately inhibit stereotypes. This was demonstrated in a study by Araya and colleagues (2003) in which 
participants had to study stereotypical (i.e., immigrant words) and non-stereotypical words, either with an initially activated social category (immigrant prime) or with no social category (neutral prime). Participants were then instructed to either forget or remember the studied words. This was followed by a recognition test. Participants in the immigrant, but not in the neutral prime condition, more often falsely recognized non-studied stereotypical words, but only when they had previously been given instructions to forget them.

\section{Urgent questions}

In recent years, a vast amount of research explored whether certain personality traits may be related to source misattribution and pseudo-memories. Thus, traits like dissociation, suggestibility, and imagery vividness have been studied to find out whether they modulate performance in pseudo-memory paradigms (e.g., Candel, Merckelbach, \& Kuijpers, 2003; Eisen \& Lynn, 2001; Geraerts, Smeets, Jelicic, van Heerden, \& Merckelbach, 2005; Horselenberg et al., 2000; Winograd, Peluso, \& Glover, 1998). On the other hand, the connection between these traits and pseudo-memories is far from robust (e.g., Wright, Startup, \& Mathews, 2005). That is, some studies were unable to find a significant relation between dissociation and pseudo-memories (Horselenberg et al., 2000). Thus, while research along these lines has yielded important clinical insights, its theoretical contribution is limited. As we have noted so far, focusing on neurocognitive concepts may explain why certain traits are related to pseudo-memories and, therefore, this research line might be fruitful. Indeed, recent aging and lesion studies suggest that neurocognitive dysfunctions play a critical role in the creation of pseudo-memories. Given this considerable amount of aging and clinical research, it is surprising how few studies have explored whether individual differences in neurocognitive tasks in young adults influence the likelihood of pseudo-memories. This is not only of theoretical interest, but also of practical importance (e.g., the accuracy of eyewitness testimonies). It is to these issues that we now turn.

Secondly, specific questions about the relationship between neurocognitive functions and pseudo-memories in clinical samples need to be answered. One issue still open to debate is whether established neurocognitive dysfunctions in schizophrenia relate to the increased susceptibility for source misattributions. To address this issue, apart from carrying out studies in schizophrenic populations, a commonly used research strategy is to examine to which extent normal samples with schizophrenia-like characteristics (i.e., schizotypy) exhibit similar deficits. That is, whether subtle neurocognitive deficits are present in this sample and whether they relate to certain memory aberrations.

As discussed earlier, the primary role of the prefrontal cortex is evaluating and monitoring relevant information and inhibiting irrelevant information. Inefficient monitoring may lead to source misattributions, such that plausible, but fictitious events are believed to be true. The next step is the acceptance of these beliefs as 
genuine memories (i.e., true recollections). Until now, research has only focused on monitoring deficits involving plausible events like remembering words never presented, or imagining and performing simple actions. The question arises whether these monitoring deficits would also arise in specific groups of people who report memories of events that seem very implausible to have happened to begin with.

\section{Outline of this thesis}

This thesis aims to explore new directions in the neuropsychology of pseudo-memories. It consists of three parts:

\section{PART [A] NORMATIVE DATA AND PSYCHOMETRILS}

The first part of this dissertation describes the standardization and validation of a pseudo-memory paradigm and a neurocognitive task. They served as a starting point for conducting our first empirical study relating suboptimal executive functioning to pseudo-memories (see Chapter 4). More specifically, Chapter 2 describes the development, standardization, and normative data of a Dutch version of the Deese/Roediger-McDermott paradigm (Deese, 1959b; Roediger \& McDermott, 1995) in a sample of Dutch undergraduate students. Although this is a standard paradigm to elicit pseudo-memories in experimental settings, a version based on Dutch word association norms was still lacking.

As described above, deficits in inhibitory control mediated by the prefrontal cortex may play a crucial role in pseudo-memories. In order to test this, a psychometrically-sound neurocognitive task measuring inhibitory control is a prerequisite. With this in mind, Chapter 3 summarizes the results of a psychometric study on a specific inhibition measure, namely the Random Number Generation task (RNG; Ginsburg \& Karpiuk, 1994, 1995).

\section{PART [B] SOURGE MONITORING, PSEUDO-MEMORIES, AND NEUROGOGNITIVE FUNGTIONS IN UNDERGRADUATES}

In this part, the links between suboptimal neurocognitive dysfunctions, source misattribution, and pseudo-memories were examined in undergraduate participants. To this end, empirical studies using several memory distortion and pseudo-memory paradigms and neurocognitive tasks, are summarized. Chapter 4 explored whether individual differences in the ability to inhibit cognitive schemas (measured by the RNG task) are related to recalling or recognizing words never presented as measured by the DRM paradigm.

Similarly, the study described in Chapter 5 examined whether undergraduates who differ in simple and complex working memory capacity, also differ in their proneness to (develop) pseudo-memories.

Chapter 6 examined whether complex working memory capacity and schizophrenia-related symptomatology in an undergraduate population were related to 
source misattributions in a high processing demand environment. In this study, participants had to fill out a psychosis-proneness questionnaire and were subjected to an action source monitoring paradigm. A task measuring complex working memory capacity was also included in this study.

In Chapter 7, we investigated whether an active manipulation of inhibitory control may lead to an increase in source misattributions of to-be-suppressed information. As described above, instructions not to think about (i.e., actively inhibit) stereotypes after stereotype priming may lead to an automatic rebound, i.e., more thinking about stereotypes. However, until now, there are no studies investigating the effects of these manipulations on the accuracy of eyewitness memory. This was addressed in Chapter 7. Here, we were primarily interested in whether active inhibition of stereotypes would rebound on a later memory task and would also lead to stereotype-consistent pseudo-memories.

\section{PART [G] NEUROCOGNITIVE DYSFUNGTIONS, SOURCE MONITORING, AND PSEUDO-MEMORIES IN GLINIGAL POPULATIONS}

The final part describes two studies addressing the relationships between neurocognitive dysfunctions, source monitoring, and pseudo-memories in clinical populations. More specifically, in Chapter 8 we investigated whether executive dysfunctions could explain certain memory aberrations (correct recognition, pseudo-memories, and source misattributions) in schizophrenic patients and matched controls.

As discussed earlier, the primary role of the prefrontal cortex is evaluating and monitoring relevant information and inhibiting irrelevant information. Inefficient monitoring may lead to source misattributions, such that plausible but fictitious events are taken as real events. The following step is the acceptance of these beliefs as genuine memories (i.e., veridical recollections). A question that arises is whether these monitoring deficits would also occur in specific groups of people who report memories of events that seem very implausible to have actually happened. Chapter 9 therefore describes a study in which we investigated whether individuals with previous-life beliefs and memories are more prone to make source misattributions, as compared to controls with no such beliefs or memories.

Finally, in Chapter 10 the results of our work are summarized, discussed, and some final conclusions are drawn. To further illustrate our findings, some new data on the link between pseudo-memory and neurocognitive function are briefly presented. Directions for future research are also discussed.

It should be noted that most of the studies that will be described in this thesis will be correlational in stead of directly testing causal relationships. Thus, most of our studies are not a direct test of the causal model described in FGURE 1.1. However, causal relationships always presume correlation. In this respect, it would be unnecessary to look for causal relationships when no relation is identified in the first place. Most of our studies can be seen as a first exploration in identifying this first necessary prerequisite of causal relationships. 





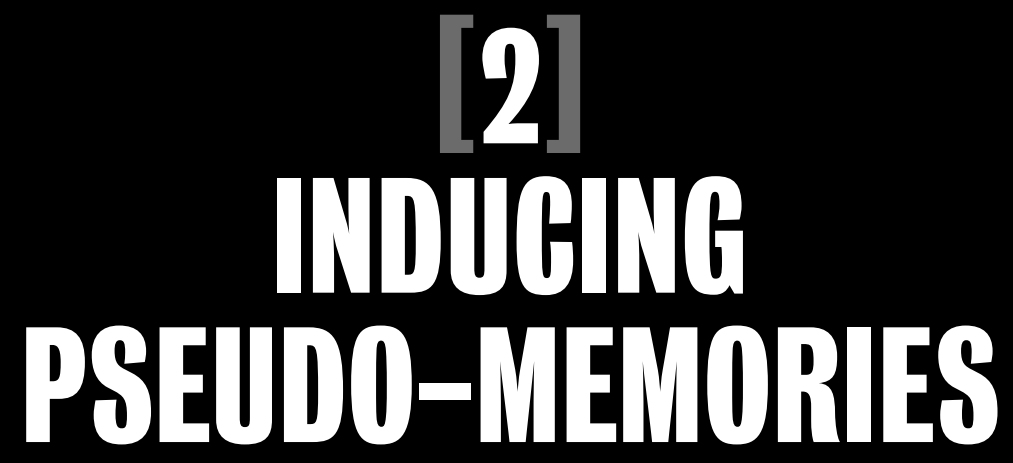

A Dutch version of the Deese/Roediger-McDermott paradigm 



\title{
CHAPTER [2] INDUGING PSEUDO-MEMORIES
}

\section{A Dutch version of the Deese/Roediger-McDermott paradigm}

An adjusted version of this chapter is submitted as: Peters, M.J.V., Jelicic, M., \& Merckelbach, H. (submitted). Inducing false memories: A Dutch version of the Deese/Roediger-McDermott paradigm.

\begin{abstract}
The Deese/Roediger-McDermott paradigm (Deese, 1959b; Roediger \& McDermott, 1995 ) is a widely used method to induce pseudo-memories in laboratory situations. In this paradigm, participants study lists of words that are semantically associated with a central non-presented word, called the critical lure word. A standard finding is that these lures are often falsely recalled and/or recognized. The purpose of the current study was to provide a set of Dutch normative data for 64 word lists in a sample of undergraduates $(n=70)$ : 32 twelve-item word lists were derived from Stadler, Roediger, and McDermott (1999), and 32 fifteen-item word lists were based on Dutch word association normative data. Rates of falsely recalling and recognizing the critical lure varied widely across lists, ranging from a high $72 \%$ of participants who recalled the critical lure word NAALD ("needle") after studying a 12-item list, to a low $0 \%$ of participants who recognized the critical lure HEMD ("shirt") after studying a 12-item list.
\end{abstract}




\section{Introduction}

Our memories are far from perfect. Sooner or later, most of our experiences will be forgotten, and the relatively few events we do remember, may differ from how they actually took place. Often, there are only minor discrepancies between actual events and our memories, but sometimes we remember events that never happened in reality (e.g., Loftus, 1993; Loftus, Miller, \& Burns, 1978). In such cases, people are said to have "false" memories or in our terminology pseudo-memories. In the past decades, researchers have developed laboratory methods to study pseudo-memories. One of these methods is the Deese/Roediger-McDermott paradigm (DRM paradigm, read DREAM; Bruce \& Winograd, 1998). This paradigm was originally developed by Deese (1959b) and revived by Roediger and McDermott (1995).

Over 40 years ago, James Deese published two complementary articles on memory quantity and accuracy (Deese, 1959a,b). The first study showed that correct free recall of a word list is directly related to the interitem associative strength of the list, i.e., the mean relative frequency with which each list item elicits all other list items as free associates. In the second study, Deese (1959b) presented 50 participants with word lists that varied in their interitem associative strength. Lists consisted of 12 highly associated words such as dream, pillow, bed, etc., all related to a non-presented critical lure word, e.g., sleep. It was found that participants often had intrusions of the non-presented lure word. For example, in recalling the 12 previously studied associates to the non-presented word sleep, $44 \%$ of the participants falsely recalled this critical lure.

Three decades later, Roediger and McDermott (1995) refined and expanded the paradigm developed by Deese. To this end, they adopted six lists that produced the most intrusions (i.e., false recall) in Deese's experiment. The authors found that on immediate free recall tests, the critical lures were recalled $40 \%$ of the time. This rate is comparable to the recall of words from the middle of the study list. To obtain additional parameters, Roediger and McDermott (1995) gave their participants a forced-choice old-new recognition test, in which studied items, critical lures, and unrelated lures were presented. Participants were also asked to rate their confidence that each presented word had occurred in the study lists (ranging from 1 "sure new" to 4 "sure old"). There were no differences in recognition between studied items and critical lures, as participants classified the critical lures as being old (.84) as often as the studied words (.86). Mean confidence rating for the critical lures (3.3) was similar to ratings for the studied words (3.6). In their second experiment, the test material involved 18 newly created lists, along with the 6 lists used in experiment 1 . Furthermore, list length was expanded with word lists now consisting of 15 words. During the recognition test, participants were asked to make meta-memory judgments (remember-know) to qualify their "old" recognition responses, as proposed by Tulving (1985) and Gardiner (1988). In Roediger and McDermott's (1995) second experiment, participants recalled the critical lure on $55 \%$ of the lists, a rate that is even higher than 
that in experiment 1. Moreover, participants were just as likely to report remember judgments for critical lures (.48) as they were for studied words (.49).

Many authors have taken false recall and/or recognition of the critical lure in the DRM paradigm as a prototypical example of pseudo-memories. Meanwhile, there are important differences between pseudo-memories as elicited by the DRM procedure and those that occur outside the laboratory (Pezdek \& Lam, 2007; Smeets, Merckelbach, Horselenberg, \& Jelicic, 2005). On the other hand, over the past decade, many studies have demonstrated the robustness of the DRM paradigm in inducing pseudo-memories, even when substantial changes are made to the original method (see for reviews Roediger \& McDermott, 2000). Different procedures have been applied in trying to reduce the probability of false recall and recognition like stimulus presentation format (e.g., Gallo, McDermott, Percer, \& Roediger, 2001), presentation modus of lists (blocked vs. random; forward vs. backward; Toglia, Neuschatz, \& Goodwin, 1999), practice (Seamon et al., 2003), number of associates per list (Robinson \& Roediger, 1997), list presentation rate (McDermott \& Watson, 2001), multiple study-test trials and list repetition (Benjamin, 2001; Kensinger \& Schacter, 1999), and focused and divided attention (Pérez-Mata, Read, \& Diges, 2002; Seamon et al., 2003). However, even in situations where participants are explicitly warned against making these pseudo-memory intrusions, false recall and recognition rates remain high (e.g., Gallo, Roberts \& Seamon, 1997; Neuschatz, Benoit, \& Payne, 2003). Furthermore, reinforcement (e.g., paying money for correct responses and taking money away for recalling critical lures) does affect memory performance, but does not eliminate false recall of the critical lures (Candel, Merckelbach, Jelicic, \& Jentjens, 2006).

Apart from parametric studies that looked at the methodology of the paradigm as described above, the DRM paradigm has also been used in special populations. For example, with this paradigm, neurologically intact elderly tend to make more false recall and recognition critical lure intrusions than younger adults (e.g., Butler, McDaniel, Dornburg, Roediger, \& Price, 2004; Dehon \& Brédart, 2004; Kensinger \& Schacter, 1999). The DRM paradigm has also been employed to study pseudo-memories in various clinical groups. With the exception of amnesic patients (Melo, Winocur, \& Moscovitch, 1999), pseudo-memories elicited by the DRM paradigm appear to be remarkably robust across fairly diverse clinical populations, including individuals with schizophrenia (Moritz, Woodward, Cuttler, Whitman, \& Watson, 2004), women with possible recovered memories of childhood abuse (Clancy, Schacter, McNally, \& Pitman, 2000; Geraerts, Smeets, Jelicic, van Heerden, \& Merckelbach, 2005), and individuals with Dementia of the AlzheimerType (DAT; see Marsh, Balota, \& Roediger, 2005).

Other authors have focused on individual differences in susceptibility to pseudo-memories in the DRM paradigm. Recently, Watson, Bunting, Poole, and Conway (2005) reported that, relative to controls, participants with poorer working memory 
capacity more often falsely recalled the critical lures, but only so when they had received a warning concerning the purpose of the associative lists (see also Chapters 4 and 5 of this dissertation).

To our knowledge, the DRM paradigm has been primarily used in English speaking participants. Moreover, relatively few studies examining pseudo-memories in other languages (e.g., Brédart, 2000 for French; Pérez-Mata et al., 2002 for Spanish; Zeelenberg \& Pecher, 2002; Zeelenberg, Plomp, \& Raaijmakers, 2003 for Dutch; Johansson \& Stenberg, 2002 for Swedish) almost all translated the original Roediger and McDermott (1995) or Stadler, Roediger, and McDermott (1999) word lists into another language (but see Anastasi, De Leon, \& Rhodes, 2005). Given the popularity of the DRM paradigm as a method to study pseudo-memories under laboratory conditions (Roediger \& McDermott, 2000), we reasoned that a well documented Dutch version of the paradigm would be in order. The main objective of the current study was to construct a Dutch version of the Deese/Roediger-McDermott paradigm, with the same list specifications as in the original DRM paradigm (i.e., lists consisting of the strongest associates to a critical lure and arranged in descending order of associative strength).

Following the methodology of Roediger and McDermott (1995), we construed 64 word lists. Half of them were drawn from Stadler et al. (1999). For these lists, 12item word lists were translated into Dutch. The other 32 lists consisted of 15 words and were based on Dutch word association norms (15-item word lists; Lauteslager, Schaap, \& Schievels, 1986; Van Loon-Vervoorn, \& van Bekkum, 1991; van der Madevan Bekkum, 1973). Below, we report normative data of false recall and recognition for these lists.

\section{Method}

\section{Participants}

Participants were 72 undergraduate psychology students (18 men) at Maastricht University who agreed to take part in the study in return for course credits. Mean age of the participants was 19.10 years ( $S D=2.52$; Range: $18-33$ ). All participants were native Dutch speakers. The study was approved by the standing ethical committee of the Faculty of Psychology of Maastricht University.

\section{Materials}

In this normative study, 64 lists were tested. Thirty-two lists consisted of 12 associates of a critical lure and 32 lists consisted of 15 associates. The 32 critical lure words are taken from Stadler et al. (1999). ${ }^{1}$ For the 12 -item word lists, associative words for each critical lure were drawn from Stadler et al. (1999). For these lists, the first 12 associates to each list were translated into Dutch. When different translations were possible, the translation was chosen that was thought to be most appropriate in eliciting a pseudo-memory. 
For the thirty-two 15-item word lists, associates were generally the first 15 items from the Dutch word association norms. The Van Loon-Vervoorn and van Bekkum (1991) normative data were used as a primary source for the normative lists. When normative word lists for the critical lures were not provided by this normative study, the Lauteslager et al. (1986) and van der Made-van Bekkum (1973) normative studies were used. Thus, for each critical lure word, a list of semantically related words was obtained. Each of the lists was arranged in descending order of associative strength.

The 64 lists were pseudo-randomly divided into four sets of 16 lists. Each set of 16 lists consisted of eight 12-item word lists and eight 15-item word lists. To eliminate overlap between lists within each set, each word was individually checked on its appearance in another list within the same set or as critical lure in another list of the same set. If this occured, these words were substituted by less frequent words from the normative lists. A random order of the lists within each set was constructed. The 64 lists were recorded onto audio tape in a female voice who read out the words at a rate of 1 word every 2 sec.

The recognition task was constructed for each of the four sets and consisted of 96 words randomly ordered on a sheet of paper. The 96 words consisted of items from serial position 1, 8, and 10 of each of the studied lists (a total of 48 items), the 16 critical lure words, and 32 additional unrelated words taken from the lists of the three non-presented sets. Only the words that were unrelated to words in the studied lists were used for this purpose.

\section{Procedure}

Participants were tested individually. They were told that they took part in a study on the efficacy of memory functioning. Each participant was presented with one of the four sets (i.e., 16 lists per participant). Participants were pseudo-randomly allocated to one of the four sets, so that each set was administered to 18 participants. Instructions given to the participants were based on the Roediger and McDermott (1995) procedure. Basically, they were told that they were going to hear several word lists and that they would be tested immediately after each list by writing the words on successive pages of examination booklets. As in the Roediger and McDermott study, participants were encouraged to write down all of the words they could remember, but only so when they were reasonably sure that the word had appeared in the list (i.e., they were told not to guess). The lists were read out loudly one after the other. The words (spoken by a female voice) were presented for $1 \mathrm{sec}$ with an inter-stimulus interval of $1 \mathrm{sec}$. Before each list, the experimenter said "List 1, List 2," and so on. At the end of each list presentation, participants were given 2 min to write down all the words they could remember.

After finishing the free recall task, participants were asked to turn their recall packets face down. Next, they were given a 5 min filler task, consisting of mental arithmetic problems. A total of 70 exercises were given to each participant, with an 
equal distribution of addition, subtraction, multiplication, and division exercises.

Following the filler task, subjects were told they would receive another test. They were then given the old-new recognition task. For each of these 96 words, participants had to indicate whether the word was old (i.e., had appeared on one of the 16 lists) or new. They were asked to read each item carefully, and only to respond "old" if the word had appeared in the presented lists. Participants worked through the recognition task at their own pace. In addition, they were asked to make remember-know judgments for each item they classified as old (Tulving, 1985). Participants were told that a remember judgment should be made for items for which they had a vivid memory of the actual presentation, e.g., when they remembered something distinctive in the speakers voice, or remembered the words presented before or after it, or remembered what they were thinking when they heard the word. Participants were instructed that know judgments were to be reserved for items that they were sure had been presented, but for which they lacked the feeling of remembering the actual occurrence of the word. It was emphasized that participants should make remember-know judgments about words with respect to their presentation on the audiotape, and not whether they remembered or knew they had written it down during the free recall tests. In addition, they were instructed to make remember-know judgments immediately after judging the item to be old and before they considered the next test item. When they had completed the recognition task, they were invited to write down what they thought was the purpose of the study. Finally, participants were thanked for their participation and debriefed.

\section{Results}

Our analyses are based on data from 70 participants. Two participants were excluded from the analyses because after the experiment, they admitted to possess knowledge of the DRM procedure. Alpha was set at .05, unless specified otherwise.

Before collapsing the data obtained with the 64 word lists, the four sets of 16 word lists were compared with regard to their potency to elicit studied words and the critical lure words. Four one-way Analyses of Variance (ANOVA's) with four levels (i.e., four sets) showed that sets did not differ in studied words that were recalled or recognized [both $F^{\prime} s(3,66)<1$, both $p$ 's $>.05$ ] or in critical lure words recalled or recognized [both $F^{\prime} s(3,66)<2$, both $p$ 's $>.05$ ] by the participants. Therefore, we pooled the data across sets. 


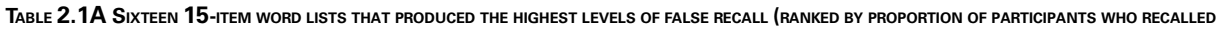
the critical lure: T; right column). Proportions of participants who correctly recalled items as a function of SERIal Position (1-15) are ALSO SHOWN.

\begin{tabular}{|c|c|c|c|c|c|c|c|c|c|c|c|c|c|c|c|c|}
\hline List & 1 & 2 & 3 & 4 & 5 & 6 & 7 & 8 & 9 & 10 & 11 & 12 & 13 & 14 & 15 & $T$ \\
\hline Needle & .83 & 1.0 & .50 & .56 & .67 & .44 & 1.0 & .67 & .44 & .33 & .61 & .61 & .78 & .72 & .94 & .61 \\
\hline Soft & .67 & .89 & .11 & .83 & .78 & .67 & .28 & .44 & .44 & .44 & .72 & 1.0 & .72 & .78 & 1.0 & .61 \\
\hline Rough & .78 & .56 & .72 & .83 & .22 & .67 & .67 & .50 & .67 & .39 & .28 & .44 & .50 & .78 & 1.0 & .61 \\
\hline Thief & .33 & .67 & .56 & .44 & .72 & .28 & .67 & .67 & .72 & .17 & .50 & .67 & .61 & .83 & .94 & .61 \\
\hline Bread & .94 & .44 & .89 & .67 & .56 & .72 & .89 & .44 & .28 & .83 & .89 & .89 & .78 & .83 & 1.0 & .55 \\
\hline Cold & .89 & 1.0 & .78 & .28 & .61 & .50 & .44 & .39 & .72 & .56 & .61 & .72 & 1.0 & .78 & 1.0 & .44 \\
\hline Angry & .78 & .94 & .83 & .33 & .06 & .56 & .56 & .44 & .72 & .28 & .78 & .72 & .28 & .83 & .56 & .44 \\
\hline Sweet & .94 & .78 & .50 & .78 & .72 & .89 & .50 & .50 & 1.0 & .39 & .39 & .89 & .72 & .33 & .83 & .44 \\
\hline Music & .89 & .83 & .72 & .67 & .44 & .17 & .56 & .72 & .50 & .83 & .72 & .50 & .89 & .78 & .94 & .44 \\
\hline Smoke & .89 & .94 & .72 & .83 & .89 & .67 & .39 & .28 & .50 & .33 & .61 & .50 & .56 & .56 & .94 & .38 \\
\hline Car & .78 & .56 & .56 & .44 & .83 & .56 & .61 & .50 & .56 & .72 & .67 & .78 & .83 & .72 & 1.0 & .33 \\
\hline Foot & .94 & .72 & .50 & .67 & .44 & .28 & .89 & .67 & .72 & .83 & .89 & .67 & .78 & .94 & 1.0 & .33 \\
\hline Chair & .78 & .72 & .78 & .72 & .50 & .44 & .67 & .33 & .44 & .61 & .78 & .61 & .50 & .83 & 1.0 & .28 \\
\hline Pen & 1.0 & .72 & .89 & .72 & .33 & .78 & .61 & .61 & .39 & .61 & .39 & .72 & .61 & .61 & .89 & .28 \\
\hline Window & .89 & .78 & .50 & .83 & .50 & .50 & .56 & .44 & .56 & .67 & .67 & .72 & .72 & .78 & .89 & .28 \\
\hline Man & 1.0 & .61 & .39 & .61 & .50 & .44 & .28 & .78 & .56 & .56 & .89 & .89 & .44 & .72 & 1.0 & .22 \\
\hline
\end{tabular}

\section{Recall and recognition}

Recall data are presented in taBLEs 2.1A, 2.1B, 2.2A, and 2.2B with lists sorted according to the proportion of false recall of the critical lure word. TABLEs 2.1A and 2.1B show the 32 lists (sixteen 15-item word lists and sixteen 12-item word lists) for which the highest levels of false recall were obtained, whereas taBLEs $2.2 \mathrm{~A}$ and 2.2B present the 32 lists (sixteen 15-item word lists and sixteen 12-item word lists) that produced the lowest false recall levels.

Table 2.1B Sixteen 12-item word lists that produced the highest levels of false recall (ranked by proportion of participants who recalled the critical lure: T; right column). Proportions of participants who correctly recalled items as a function of serial position (1-12) ARE ALSO SHOWN.

\begin{tabular}{|c|c|c|c|c|c|c|c|c|c|c|c|c|c|}
\hline LIST & 1 & 2 & 3 & 4 & 5 & 6 & 7 & 8 & 9 & 10 & 11 & 12 & $T$ \\
\hline Needle & .94 & .61 & .56 & .50 & .89 & .61 & .72 & .50 & .67 & 1.0 & .83 & 1.0 & .72 \\
\hline Rough & .94 & .56 & .33 & .61 & .56 & .61 & .72 & .39 & .28 & .50 & .78 & .94 & .61 \\
\hline Pen & 1.0 & .72 & .83 & .67 & .56 & .78 & .39 & .56 & .72 & .78 & 1.0 & 1.0 & .50 \\
\hline Soft & .83 & .50 & .39 & .72 & .28 & .61 & .17 & .44 & .56 & .50 & .72 & .89 & .50 \\
\hline Sweet & .89 & .67 & .72 & .83 & .67 & .72 & .50 & .28 & .61 & .89 & .94 & .94 & .50 \\
\hline Black & .83 & .89 & .61 & .78 & .67 & .33 & .50 & .78 & .61 & .94 & .89 & 1.0 & .39 \\
\hline Cold & .94 & .83 & .83 & .89 & .83 & .61 & .39 & .56 & .72 & .50 & .94 & .83 & .39 \\
\hline Window & .83 & .78 & .61 & .83 & .78 & .67 & .56 & .83 & .67 & .94 & .89 & 1.0 & .33 \\
\hline Angry & .83 & .72 & .83 & .56 & .28 & .17 & .67 & .39 & .72 & .83 & .61 & .83 & .28 \\
\hline Foot & .83 & .89 & .83 & .72 & .89 & .44 & .89 & .44 & .83 & .72 & .72 & 1.0 & .28 \\
\hline Smoke & .94 & .83 & .72 & .61 & .67 & .61 & .28 & .50 & .56 & .78 & .89 & .83 & .28 \\
\hline Mountain & .72 & .89 & .56 & .83 & .72 & .61 & .61 & .56 & .67 & .72 & .89 & .89 & .28 \\
\hline Car & 1.0 & .50 & .94 & .44 & .61 & .61 & .22 & .61 & .83 & .83 & .72 & 1.0 & .22 \\
\hline City & .83 & .50 & .94 & .94 & .67 & .17 & .61 & .83 & .67 & .67 & 1.0 & 1.0 & .22 \\
\hline Cup & .78 & .89 & .83 & .83 & .67 & .56 & .44 & .83 & .50 & .89 & .67 & .83 & .22 \\
\hline Chair & .94 & .94 & .56 & .72 & .67 & .78 & .78 & .50 & .39 & .89 & 1.0 & .78 & .22 \\
\hline
\end{tabular}


Proportions of participants who falsely recalled the critical lure varied widely across lists, with the highest proportions being .72 for participants who had the 12item word list NAALD ("needle"), to .00 for participants who had the 12-item word lists DOKTER ("doctor"), SLAAP ("sleep"), SPIN ("spider") and HEMD ("shirt"). Across all 64 lists, the mean proportion of participants who falsely recalled the critical lure was .26. In the Stadler et al. (1999; see also Roediger \& McDermott, 1995), it was found that the mean proportion of participants falsely recalling the lure came close to the proportion of participants who recalled an item in the mid-list position (positions 4-11).

Table 2.2A Sixteen 15-item word lists that produced the lowest level of false recall (Proportion of participants who recalled the Critical lure: T; right column). Proportions of participants who correctly recalled studied items as a function of SERIal position (1-15) ARE ALSO SHOWN.

\begin{tabular}{|c|c|c|c|c|c|c|c|c|c|c|c|c|c|c|c|c|}
\hline LIST & 1 & 2 & 3 & 4 & 5 & 6 & 7 & 8 & 9 & 10 & 11 & 12 & 13 & 14 & 15 & $\mathrm{~T}$ \\
\hline Lion & .94 & .72 & .50 & .67 & .44 & .28 & .89 & .67 & .72 & .83 & .89 & .67 & .78 & .94 & 1.0 & .20 \\
\hline River & .94 & .72 & .50 & .61 & 1.0 & .78 & .72 & .44 & .83 & .56 & .83 & .78 & .83 & .67 & .94 & .20 \\
\hline Doctor & .89 & .56 & .89 & .50 & .56 & .61 & .61 & .56 & .44 & .67 & .61 & .67 & .83 & .78 & 1.0 & .17 \\
\hline Fruit & 1.0 & .78 & .56 & .39 & .78 & .67 & .78 & .78 & .61 & .89 & .72 & .61 & .83 & .89 & .83 & .17 \\
\hline King & .94 & .72 & .78 & .44 & .39 & .50 & .44 & .94 & 1.0 & .72 & .72 & .83 & .89 & .89 & .94 & .17 \\
\hline Cup & .89 & .78 & .83 & .50 & .61 & .39 & .67 & .72 & .61 & .67 & .67 & .61 & .67 & .94 & .94 & .17 \\
\hline Trash & .78 & .50 & .61 & .78 & .61 & .50 & .72 & .67 & .67 & .39 & .78 & .22 & .94 & .83 & .56 & .17 \\
\hline Black & 1.0 & 1.0 & .61 & .28 & .17 & .61 & .44 & .56 & .56 & .44 & .72 & .39 & .78 & .94 & .89 & .11 \\
\hline Girl & .94 & .83 & .72 & .89 & .89 & .44 & .72 & .50 & .50 & .78 & .67 & .78 & .78 & .89 & .94 & .11 \\
\hline Mountain & 1.0 & .83 & .94 & .83 & .94 & .72 & .28 & 1.0 & .44 & .56 & .28 & .56 & .33 & .94 & .94 & .11 \\
\hline Shirt & .83 & 1.0 & .67 & .78 & .83 & .83 & .72 & .78 & .67 & .61 & .61 & .61 & .89 & .89 & .94 & .11 \\
\hline Smell & .67 & .94 & .61 & .78 & .67 & .89 & .83 & .50 & .56 & .67 & .72 & .89 & .39 & .78 & 1.0 & .11 \\
\hline Sleep & 1.0 & .94 & .94 & .50 & .83 & .67 & .56 & .44 & .78 & .44 & .39 & .61 & .72 & .78 & .89 & .11 \\
\hline Spider & .78 & .78 & .50 & .22 & .72 & .56 & .56 & .33 & .78 & .67 & .78 & .61 & .89 & .72 & .94 & .11 \\
\hline Army & .94 & .89 & .83 & .78 & .61 & .78 & .50 & .50 & .67 & .33 & .50 & .50 & .72 & .89 & 1.0 & .05 \\
\hline City & 1.0 & .78 & 1.0 & .50 & .28 & 1.0 & .83 & .44 & .78 & .78 & .44 & .56 & .67 & .61 & 1.0 & .05 \\
\hline
\end{tabular}

As can be seen in figure 2.1, the serial position curve shows clear recency and primacy effects in recalling the studied words. However, contrary to the findings of Roediger and McDermott, the overall mean proportion of participants falsely recalling the critical lure $(M=.26)$ was far below the proportion who correctly recalled items in the mid-list position (positions $4-11 ; M=.64$ ). When list length is taken into account, mean proportion of participants correctly recalling studied words in the mid-list position was .60 for the 15-item word lists (using positions $4-11$ ) and .63 for the 12-item word lists (using positions 4-8), with proportions for the recalling of the critical lure being .29 for the 15-item word lists and .23 for the 12-item word lists. For the 16 lists that produced the highest false recall in the 15-item (TABLE 2.1 A) and 12-item (TABLE 2.1 B) word lists, mean proportions of participants recalling the critical word were .43 and $.37 ; t(30)=1.11, p$ $=.23$. Thus, in the top lists, 15 -item word lists did not induce more false recall than 12 item word lists. When only the top 16 lists (across the 12- and 15-item word lists) were taken into account, mean proportion of participants falsely recalling the critical lure word was .52. These findings are in accordance with those of Roediger and McDermott (1995) and Stadler et al. (1999). Overall proportion of veridical recall for the 12-item word lists was .68 compared to .63 for the 15 -item word lists. 


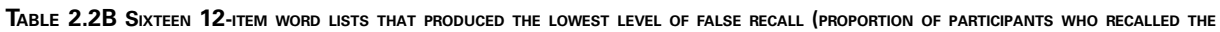
Critical lure: T; right column). Proportions of participants who correctly recalled studied items as a function of Serial position (1-12) ARE ALSO SHOWN.

\begin{tabular}{|c|c|c|c|c|c|c|c|c|c|c|c|c|c|}
\hline LIST & 1 & 2 & 3 & 4 & 5 & 6 & 7 & 8 & 9 & 10 & 11 & 12 & $T$ \\
\hline Music & .83 & 1.0 & .78 & .44 & .89 & .50 & .89 & .78 & .56 & .50 & .83 & .94 & .20 \\
\hline Bread & 1.0 & .89 & .61 & .56 & .50 & .78 & .72 & .78 & .44 & .72 & 1.0 & 1.0 & .17 \\
\hline Thief & .89 & .67 & .50 & .61 & .56 & .56 & .33 & .89 & .61 & .50 & .94 & 1.0 & .17 \\
\hline River & .94 & .72 & .83 & .94 & .89 & .44 & .39 & .50 & .67 & .89 & .61 & .83 & .17 \\
\hline Smell & 1.0 & .72 & .56 & .89 & .83 & .89 & .72 & .72 & .61 & 1.0 & .83 & .94 & .17 \\
\hline Army & .78 & .44 & .78 & .83 & .94 & .44 & .67 & .83 & .83 & .61 & .56 & .89 & .11 \\
\hline Girl & .94 & .89 & .50 & .83 & .61 & .44 & .78 & 1.0 & .78 & .94 & .94 & .94 & .05 \\
\hline Lion & 1.0 & .89 & .50 & .83 & .78 & .67 & .56 & .56 & .67 & 1.0 & .78 & .94 & .05 \\
\hline Fruit & .83 & .72 & .83 & .83 & .44 & 1.0 & .56 & .78 & .78 & .50 & .72 & .89 & .05 \\
\hline King & .94 & 1.0 & .78 & .56 & .72 & .78 & .61 & .61 & .94 & .94 & .89 & .94 & .05 \\
\hline Man & .94 & .83 & .78 & .78 & .61 & .94 & .56 & .67 & .78 & .83 & .44 & .89 & .05 \\
\hline Trash & .89 & .72 & .67 & .28 & .56 & .44 & .28 & .56 & .56 & .72 & .83 & .94 & .05 \\
\hline Doctor & 1.0 & .89 & 1.0 & .61 & .56 & .83 & .72 & .72 & .67 & .72 & 1.0 & .94 & .00 \\
\hline Sleep & .89 & .89 & .39 & .72 & .83 & .22 & .67 & .67 & .33 & .72 & 1.0 & .94 & .00 \\
\hline Spider & .94 & .72 & .83 & .56 & .39 & .83 & .61 & .89 & .50 & .78 & .94 & .89 & .00 \\
\hline Shirt & 1.0 & .72 & .56 & .83 & .67 & .89 & .78 & .83 & .89 & .78 & .67 & 1.0 & .00 \\
\hline
\end{tabular}

Veridical recall did not correlate with false recall of the critical lure word. That is, the Pearson product moment correlation across participants $(n=70)$ between the proportion of the list items correctly recalled and the proportion of the 16 critical lures falsely recalled was $r=-.17(p=.17)$. This is in accorcance with the modest correlation $(r=.20)$ reported by Stadler et al.(1999).

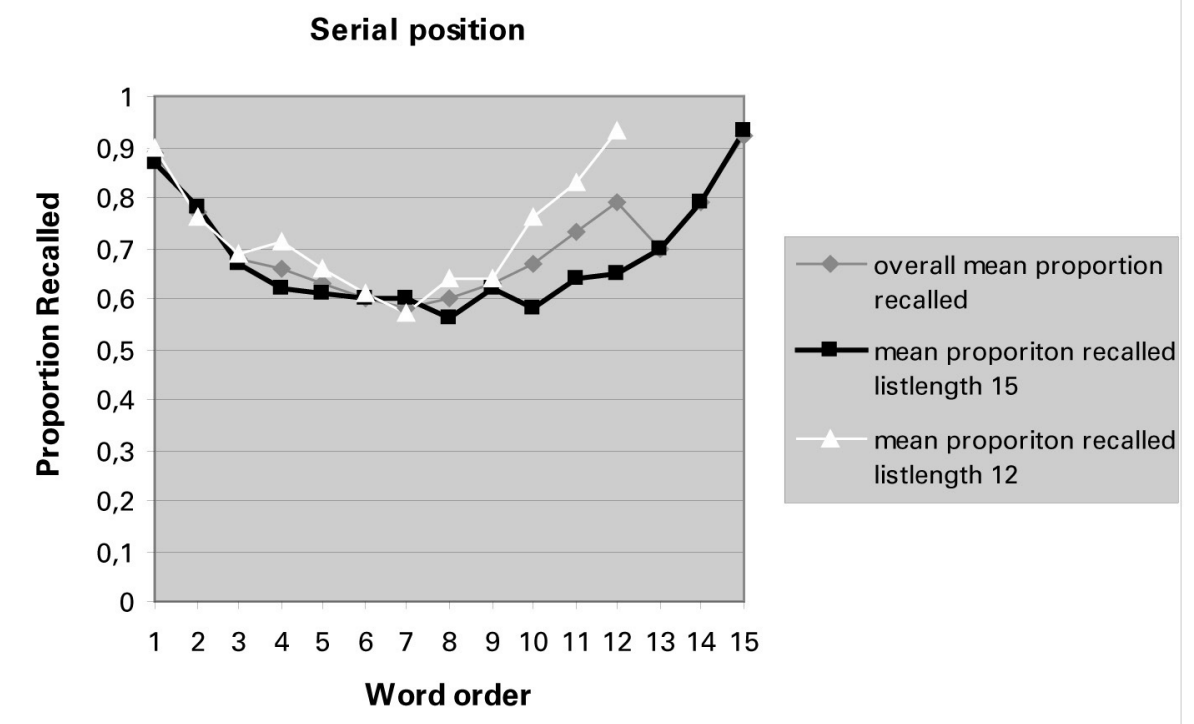

Figure 2.1 Proportion of correct recall as a function of SERIAL Position for the 64 wOrd Lists (overall Mean Proportion), the 32 Lists With List Length 15, AND the 32 Lists With List Length 12. 
TABLE 2.3 presents the recognition data for the 15-item and 12-item word lists. The lists are sorted according to the proportion of participants who falsely recognized the critical lure. Eighty-nine percent of the participants falsely recognized the word RUW ("Rough"), compared to zero percent for the word HEMD ("shirt"). The overall proportion of participants falsely recognizing the lures across all lists was .49 : .50 for the 15 -item word lists and .47 for the 12 -item word lists; $t(69)=.56, p=.58$. Mean proportion for falsely recognizing the critical lure word in the top lists was .66 for the 15 -item word lists against .62 for the 12 -item word lists; $t(30)=.80, p=.42$. For the bottom lists, these proportions were .33 for the 15-word list and .31 for the 12-word list; $t(30)=.50, p=.62$. When only the top 16 lists (across 12 - and 15-item word lists) were taken into account, the mean proportion of recognizing the critical lure word was .75 . The mean proportion of participants who correctly recognized items across all lists was .83 ( $M=.84$ for the 12 -word lists and $M=.81$ for the 15 -word lists).

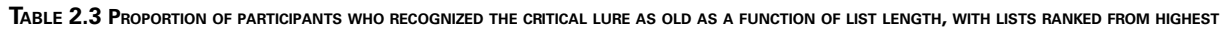
TO LOWEST PROPORTION.

\begin{tabular}{|c|c|c|c|}
\hline LIST LENGTH $=15$ & & LIST LENGTH $=12$ & \\
\hline LIST & PROPORTION & LIST & PROPORTION \\
\hline Soft & .88 & Rough & .89 \\
\hline Needle & .78 & Cold & .83 \\
\hline Cold & .78 & Needle & .76 \\
\hline Smell & .78 & Thief & .72 \\
\hline Rough & .76 & Soft & .71 \\
\hline Smoke & .76 & Black & .67 \\
\hline Thief & .71 & Smoke & .67 \\
\hline Bread & .67 & Sweet & .65 \\
\hline Sweet & .67 & Pen & .61 \\
\hline Trash & .67 & Mountain & .58 \\
\hline River & .61 & Window & .55 \\
\hline Music & .53 & Foot & .50 \\
\hline Foot & .50 & Army & .50 \\
\hline Chair & .50 & Smell & .47 \\
\hline Man & .50 & Music & .44 \\
\hline City & .50 & Bread & .44 \\
\hline Angry & .44 & Man & .42 \\
\hline Car & .44 & Sleep & .42 \\
\hline Fruit & .44 & River & .41 \\
\hline Mountain & .44 & Trash & .41 \\
\hline Sleep & .41 & Angry & .39 \\
\hline Lion & .39 & City & .39 \\
\hline Girl & .39 & Chair & .39 \\
\hline Doctor & .33 & Car & .33 \\
\hline Army & .33 & Cup & .33 \\
\hline Pen & .29 & Girl & .33 \\
\hline King & .28 & Lion & .28 \\
\hline Window & .24 & Doctor & .28 \\
\hline Cup & .22 & King & .22 \\
\hline Black & .22 & Fruit & .17 \\
\hline Shirt & .22 & Spider & .11 \\
\hline Spider & .12 & Shirt & .00 \\
\hline
\end{tabular}

As was the case for the recall data, a non-significant correlation was found between veridical recognition and false recognition. Cumulating over the lists, the correlation across participants between the proportion of the 48 list items correctly recognized 
and the proportion of the 16 critical lure words falsely recognized was $r=-.04, p=.73$. The normative study of Stadler et al. (1999) also found a non-significant correlation ( $r=.15, p$ $>$.05) between correct and false recognition.

Recall and recognition of the critical lure word were highly correlated. Across all lists, Pearson product-moment correlation between both proportions was .74 $(p<.001)$. This findings nicely corresponds with the finding of Stadler et al. (1999), who reported a similar correlation of $r=.77$. Clearly, this strong relationship is at least partly explained by the fact that the recall task preceded the recognition task.

\section{Remember/Know}

For $26 \%$ of the critical lures recognized as old, participants gave a remember judgment, while $21 \%$ of the lures were given a know judgment. For the studied words, percentage of items that were given a remember judgment was $57 \%$, while $27 \%$ received a know judgment.

When list length was taken into account, percentage of correct recognition with a remember judgment was $56 \%$ for the 15 -item word lists and $57 \%$ for the 12 -item word lists. Know judgments for correct recognition were $27 \%$ (15-item word lists) and $28 \%$ (12-item word lists). For the 15-word lists, overall proportions were $30 \%$ for remember and $21 \%$ for know judgments. For recognition of the critical lure word, overall proportion of remember judgments for the 12 -word lists was $22 \%$, against $21 \%$ for know judgments.

\section{Discussion}

The present study was initiated to develop Dutch semantically related lists and evaluate their effectiveness in eliciting false recall and recognition in a DRM paradigm. Ever since the pioneering work of Elizabeth Loftus (e.g., Loftus et al., 1978) on memory distortion, memory research has shifted from an emphasis on memory as an accurate reproduction of past experiences to memory as a reconstructive process that sometimes goes awry. Over the past two decades, many studies have examined how, when, and why people are susceptible to pseudo-memories. The DRM paradigm has contributed substantially in clarifying these issues (Roediger \& McDermott, 2000).

Although all lists were constructed in a similar way (i.e., based on association norms), it is clear that they differ in their effectiveness to produce pseudo-memories. As was found in several studies (e.g., Deese 1959b; Gallo \& Roediger, 2002), lists vary strongly in their tendency to activate the critical lure. This has to do with differences in associative strength, more specifically the degree to which all of the list words are related to each other and to the critical lure word. The current study identified a series of effective and relatively ineffective word lists in terms of their capacity to elicit false recall and recognition. The top 16 lists identified in our study yielded rates of false recall and recognition that are highly comparable to the effects described by Roediger and McDermott (1995) and Stadler and colleagues (1999). Apparently, the 16 bottom lists are those with the lowest associative strength. 
We further explored whether the number of associates in the study lists affected false recall and recognition rates. However, one should be cautious in interpreting these data, since the difference in the construction of the 12- and 15-item word lists might serve as a confounding factor. The 12-item word lists were translated from the Stadler et al. (1999) normative study, while the 15-item word lists were based on Dutch word association norms. When meta-memory responses were taken into account across all lists, we found for both correct and false recognition that the percentage of "remember" judgments exceeded "know" judgments, as was found by Roediger and McDermott (1995).

What are the origins of false recall and recognition in this paradigm and how can our normative findings best be explained? One explanation relates to Underwood's (1965) implicit associative response theory. By this view, pseudo-memories in the DRM paradigm are a byproduct of encoding. Thus, the encoding of list items during study (e.g., dream, pillow, etc.) triggers the critical lure word (e.g., sleep). When on a subsequent recognition task the lure word (e.g., sleep) is given, participants may claim to recognize the word being part of the list, due to the earlier implicit associative response. Evidence for this intriguing account comes from two research lines. Firstly, false recognition increases linearly with the number of associated items in the study lists (e.g., Robinson \& Roediger, 1997), as was found in our study. Secondly, encoding of items grouped in lists as opposed to encoding randomly mixed items promotes false recognition (e.g., Mather, Henkel, \& Johnson, 1997). However, some findings are difficult to reconcile with the associative encoding account. For example, Koutstaal and Schacter (1997) reported a study in which they had participants study pictures from different categories (e.g., shoes, cars, etc.). On a subsequent recognition task, participants falsely recognized non-presented, but category related pictures. It is unlikely that participants automatically encoded these distinctive pictures during the study phase. Koutstaal and Schacter (1997) argued that participants encode a general theme of the studied items, and lures are thus falsely recognized because they relate to this general theme (see also Miller \& Gazzaniga, 1998).

A theoretical account in line with this interpretation is the fuzzy trace theory by Reyna and Brainerd (e.g., Reyna \& Brainerd, 1995). According to this theory, a two-track process is involved in the encoding of events in memory, namely gist and verbatim. While the former represents the general theme of an event (e.g., "I know that I have eaten yesterday"), the latter refers to event-specific details such as perceptual details (e.g., "I can clearly remember the picture of having eaten fries yesterday"). The fussy trace theory implies that false recall and/or recognition are a byproduct of overreliance on gist traces. Thus, in the DRM paradigm, it is in principle possible to remember the themes of the studied lists (e.g., textiles, transport, etc.), without specifically recalling the verbatim (studied words) traces. It is retrieval of the theme that may produce recall and recognition of lures. However, this theory makes only sense in the context of DRM studies relying on lists that elicit high levels of pseudo-memories. As was found in our study, certain lists produce negligible rates of false recall and recognition, even though they are constructed to converge on a specific gist trace. Nevertheless, for reasons yet to be clarified, they do not produce false recall. 
An alternative and more suitable explanation for our findings, which is also closely related to the implicit associative response theory by Underwood (1965), is the activationmonitoring framework. This approach is based on the source monitoring framework by Johnson, Hashtroudi, \& Lindsay (1993) (see Gallo \& Roediger, 2002. It incorporates both encoding and retrieval factors. In brief, when list items are studied during the study phase (encoding), the critical non-presented associate (critical lure) will be activated. Pseudomemories reflect a failure in correct monitoring of the source of this activation during retrieval. An explanation of list length differences based on the activation-monitoring framework (Gallo \& Roediger, 2002) would be that increasing the amount of information to be encoded, makes it more difficult to correctly monitor this amount of information during retrieval. Not only is the activation of the critical lure affected by the associative strength and presentation rate (with high associative strength within a list increasing and slower presentation rates decreasing false recollections), but also by list length, such that an increase in list length is followed by a heightened susceptibility of critical lure activation (see Robinson \& Roediger, 1997).

In comparing our false recall and recognition rates ( $26 \%$ and $49 \%$, respectively) to those of other DRM studies, our rates are relatively low. In the first experiment by Roediger and McDermott (1995), non-presented critical lures were recalled by $40 \%$ of the participants. In their second experiment, false recall of the critical lure was even higher $(55 \%)$, while a false recognition rate of $76 \%$ was obtained. A likely explanation for these discrepancies in false recall rates is the variability in stimulus material to elicit recall and/ or recognition of the critical lure. In our experiment, we used 64 lists, compared to only 6 lists in the first and 24 lists in the second experiment of Roediger and McDermott (1995). When looking at our top 16 lists, we found false recall and recognition rates of $52 \%$ and $75 \%$, respectively, values that come close to those reported by Roediger and McDermott (1995) for their second experiment. So, stimulus material is an important determinant of false recall rates and, indeed, by relying on our lists that thematically circle around doctor, sleep, spider, and shirt, we could devise an experiment that produces false recall rates of zero. In a recent review, Pezdek and Lam (2007) argued that "[...] researchers often seek paradigms that are likely to produce the effect they desire [...]The DRM task is a fail-safe semantic priming task that always produces memory intrusion errors" (Pezdek \& Lam, 2007). As our data show, this conclusion does not hold and it is theoretically interesting to ask why DRM lists sometimes produce high levels of false recall and sometimes zero rates of false recall.

The DRM paradigm is a powerful tool in studying false recall and recognition within normal (e.g., Watson et al., 2005) as well as clinical populations (Moritz et al., 2004). With the normative data from our study, the road is also open for Belgian and Dutch researchers to address new and intriguing issues related to pseudo-memories thereby broaden the theoretical and applied knowledge of DRM induced memory illusions. 



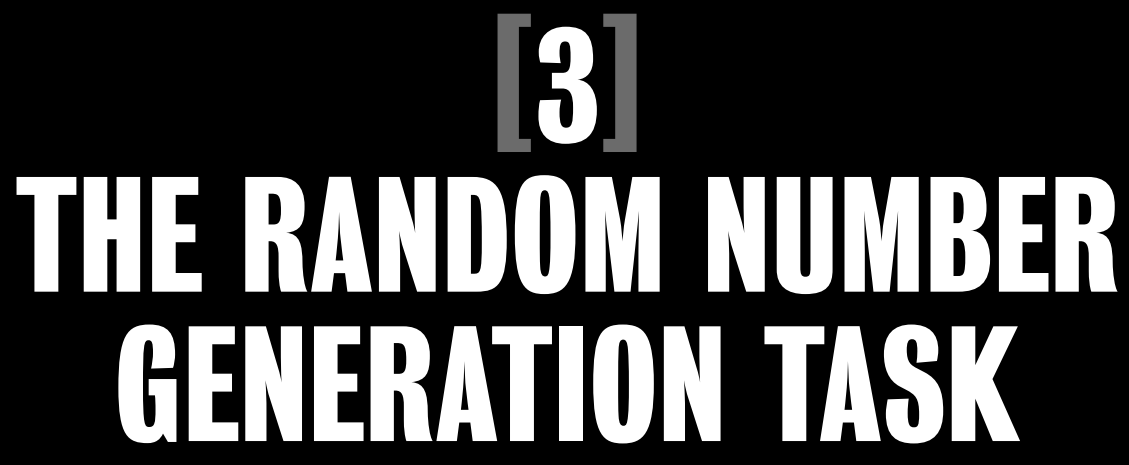

Psychometric properties and normative data of an executive function task in a mixed sample 



\title{
CHAPTER [3] THE RANDOM NUMBER GENERATION TASK
}

\section{Psychometric properties and normative data of an executive function task in a mixed sample}

An adjusted version of this chapter is accepted as: Peters, M.J.V., Giesbrecht, T., Jelicic, M., \& Merckelbach, H. (accepted). The Random Number Generation task: Psychometric properties and normative data of an executive function task in a mixed sample. Journal of the International Neuropsychological Society.

\begin{abstract}
We investigated the psychometric properties of the Random Number Generation (RNG) task in four studies using a mixed sample of young adults ( $n=306$ ), middle-aged adults $(n=40)$, and schizophrenic patients $(n=26)$. Data in study 1 were best accounted for by a three-factor solution representing inhibition of stereotypical schemas (seriation), output inhibition (repetition), and monitoring of previous output (cycling). Modest test-retest correlations were found, with the seriation factor showing acceptable stability across time (study 2). In study 3, RNG task performance was related to scores on concurrent neurocognitive tasks to establish construct validity. RNG scores correlated with healthy controls' performance on the Stroop colour-word test and with schizophrenic patients' executive dysfunctions. Schizophrenic patients performed poorer on the seriation factor of the RNG than healthy control participants (study 4). Our results indicate that the RNG task has modest to acceptable psychometric properties. It primarily taps executive subfunctions (i.e., inhibition, updating, and monitoring), which are affected by psychopathological or neurological deficits.
\end{abstract}




\section{Introduction}

When asked to generate random sequences of digits, people usually perform poorly (i.e., non-random; Wagenaar, 1972). A paradigm to measure deviations from randomness is the Random Number Generation (RNG; e.g., Ginsburg \& Karpiuk, 1994) task. In this task, participants are asked to produce sequences of digits (e.g., 1-10) in a random fashion. Successful RNG performance requires various higher order processes, including retaining task-related instructions (e.g., set size, task instructions) in memory, integrating information and holding it 'on-line' in working memory (central executive involvement; Baddeley, 1986), avoiding interference, monitoring output, and switching or modifying production strategy in accordance with the "on-line" concept of randomness (executive functioning; Baddeley, Emslie, Kolodny, \& Duncan, 1998; Jahanshahi, Saleem, Ho, Dirnberger, \& Fuller, 2006). There is convincing evidence that people's difficulties with RNG are neither attributable to a misconception of randomness nor to short-term memory problems (Baddeley, 1998; Wagenaar, 1970).

Several versions of RNG have been used (e.g., Brugger, 1997). They differ in set size (0-9, 1-20, etc.; e.g., Towse, 1998), pacing technique (paced or unpaced; e.g., Joppich, Däuper, Dengler, Johannes, Rodriguez-Fornells, \& Münte, 2004), response pace (500 msec, $1 \mathrm{sec}$, etc.; e.g., Daniels, Witt, Wolff, Jansen, \& Deuschl, 2003), response modality (oral, written, etc.; e.g., Schneider, Joppich, van der Lugt, Däuper, \& Münte, 2004), and instructions used (implicit, explicit, biased; see for a review Brugger, 1997). Despite these differences, there is broad consensus that RNG requires the allocation of central executive resources (e.g., Baddeley, 1986).

Several RNG parameters have been proposed to quantify deviations from randomness (e.g., Ginsburg \& Karpiuk, 1994; 1995; Towse \& Neil, 1998). One influential set of RNG parameters is described by Ginsburg and Karpiuk (1994). It consists of the following 9 parameters: Coupon (Cn), Gap (Gp), Poker (Pk), Runs (Rn), Repetitions $(\mathrm{Rp})$, Series (Sr), Variance of digits (VD), Digram repetition (DR), and Cluster ratio (Cr). TABLE 3.1 gives definitions of these 9 parameters. In their study, Ginsburg and Karpiuk (1994) had 32 undergraduates ( 3 men), ranging in age from 19 to 50 years $(M=30)$, produce a sequence of 100 digits consisting of the digits 0 to 9, while avoiding any system. RNG was paced by a metronome at 40 responses/min. Next, the authors performed a factor analysis (Principal Component Analysis, Varimax rotation) on the described RNG indices. This yielded three factors: seriation (loadings $\mathrm{Rn}=.84, \mathrm{DR}=$ .79 , and $\mathrm{Sr}=.77$ ), cycling (loadings $\mathrm{Gp}=.86, \mathrm{VD}=.81, \mathrm{Cn}=.66$, and $\mathrm{Pk}=.50$ ), and repetition (loadings $\mathrm{Rp}=.91, \mathrm{Pk}=.78$, and $\mathrm{Cn}=.53$ ). The three factors are interpreted as reflecting inhibition of stereotyped cognitive schemas, successful monitoring of previous output, and output inhibition, respectively (Williams, Moss, Bradshaw, \& Rinehart, 2002). 


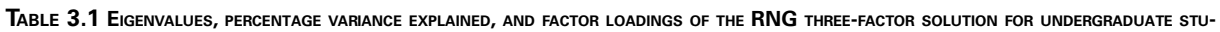
DENTS $(\boldsymbol{N}=306)$. Definitions OF THE DifFerent RNG INDICES ARE ALSO SHOWN.

\begin{tabular}{|c|c|c|c|c|}
\hline & Definition & $\begin{array}{l}\text { FActor } 1 \\
\text { SERIATION }\end{array}$ & $\begin{array}{l}\text { FACtOR } 2 \\
\text { CYycLing }\end{array}$ & $\begin{array}{l}\text { FACTOR } 3 \\
\text { REPETITION }\end{array}$ \\
\hline Eigenvalue & & 2.94 & 2.09 & 1.44 \\
\hline$\%$ Variance & & 32.61 & 23.17 & 15.97 \\
\hline \multicolumn{5}{|c|}{ Factor loadings } \\
\hline Cluster Ratio & $\begin{array}{l}\text { Variance of } 100 \text { successive responses in the digram } \\
\text { matrix, which presents the frequency with which each } \\
\text { item is followed by each of the possible other items. } \\
\text { In a series with } n \text { there are } n^{2} \text { possible parings. }\end{array}$ & .871 & --- & --- \\
\hline $\begin{array}{l}\text { Digram } \\
\text { Repetition }\end{array}$ & Number of digram repetitions (e.g., $1,5, \ldots \ldots .1,5)$ & .850 & --- & --- \\
\hline Series & $\begin{array}{l}\text { Number of consecutive digrams (e.g., } 4,5) \text {. Score } \\
\text { is calculated by summing up the number of these } \\
\text { digrams. }\end{array}$ & .803 & --- & --- \\
\hline Runs & $\begin{array}{l}\text { Number of responses in successive ascending runs. } \\
\text { The runs score is the variance of these numbers. }\end{array}$ & .756 & --- & --- \\
\hline Gap & $\begin{array}{l}\text { Measure of cycling through the set of } 10 \text { items. This } \\
\text { measure is obtained by counting the number of } \\
\text { gaps between two identical digits. The median of this } \\
\text { number is then calculated. }\end{array}$ & --- & -.849 & --- \\
\hline $\begin{array}{l}\text { Variance of } \\
\text { digits }\end{array}$ & Variance of the frequencies of each digit. & --- & .831 & --- \\
\hline Coupon & $\begin{array}{l}\text { Number of responses that occur before all digits are } \\
\text { emitted. Coupon score is the average for all complete } \\
\text { sets. }\end{array}$ & --- & .419 & --- \\
\hline Repetition & $\begin{array}{l}\text { Number of identical pairs (e.g., } 4,4) \text {. Score is the sum } \\
\text { of the number of these identical pairs. }\end{array}$ & --- & --- & .932 \\
\hline Poker & $\begin{array}{l}\text { Number of repetitions of the same digit within the } 20 \\
\text { sequences of } 5 \text { successive responses. The sum of the } \\
\text { number of sequences that contain exactly two of a } \\
\text { kind is the Poker score. }\end{array}$ & --- & --- & .903 \\
\hline
\end{tabular}

NOTE: FACTOR LOADINGS $<.40$ ARE OMITTED IN THIS TABLE

Although RNG has been widely used as a research tool in both healthy and clinical populations (Artiges et al., 2000; Brown, Soliveri, \& Jahanshahi, 1998; Brugger, Monsch, Salmon, \& Butters, 1996; Joppich et al., 2004), psychometric data (e.g., factor structure, test-retest reliability, construct validity) about this tool are scarce. With this in mind, we conducted four studies to investigate the psychometric properties of the RNG task, focusing on factor structure of the indices proposed by Ginsburg and Karpiuk (1994) (study 1), test-retest reliability and practice effects (study 2), construct validity (study 3 ), and criterion-related validity (study 4) in a mixed sample of healthy participants and clinical patients. 


\section{Study 1}

The three-factor solution proposed by Ginsburg and Karpiuk (1994) was based on a small sample $(n=32)$. Since their three-factor solution is generally in accordance with the taxonomy of response biases in human behaviour (Rabinowitz, 1970), we wanted to examine whether we could replicate the Ginsburg and Karpiuk (1994) solution, now using a more appropriate sample size for conducting factor analysis.

\section{Method}

\section{Participants}

A group of 306 (98 men) undergraduate psychology students participated in this study in return for course credits. Age ranged from 17 to 54, with a mean age of 19.90 $(S D=4.37)$. None of the participants had a history of alcoholism, head injury, psychiatric illness, or a neurological condition. The study was approved by the standing ethical committee of the Faculty of Psychology, Maastricht University. Note that the data described in this manuscript was obtained in compliance with the regulations of our institution and human research was completed in accordance with the Helsinki Declaration (http://www.wma.net/e/policy/b3.htm).

\section{Materials and Procedure}

Participants were tested individually. Upon arrival in the laboratory, they signed an informed consent form and were administered the RNG task. The RNG task was taken from Towse (1998), with the exception of response pace, which was set at one digit per sec (indicated by a metronome adjusted to $60 \mathrm{bpm}$ ). This was done to increase comparability with other factor analytic studies (e.g., Miyake, Friedman, Emerson, Witzki, Howerter, \& Wager, 2000) and studies relying on similar samples (e.g., Brugger, Pietzsch, Weidmann, Biro, \& Alon, 1995). More specifically, participants were asked to generate a random sequence of digits (set size: 1-10), for a period of $100 \mathrm{sec}$. The concept of randomness was explained using the instruction of Baddeley (1966), which draws an analogy of picking digits out of a hat, reading them loud, putting them back and then picking the next digits from the hat (see also Towse, 1998). Our instruction emphasized that a random sequence would not contain a preponderance of repetitions or adjacent number values. ${ }^{1}$ Participants were told to say out loud one digit with each tone. They were told that if their response rate fell behind the required pace, they were to listen to the rhythm and speed up their response time accordingly.

\section{Data analysis}

The 9 RNG indices (Ginsburg \& Karpiuk, 1994), including cluster ration (CR), were calculated (cf. supra).These 9 indices were subjected to Principle Component Analysis (PCA) with an orthogonal (varimax) as well as an oblique rotation (direct oblimin), because we did not know whether the extracted factors would correlate with each other (oblique) or not (orthogonal). 
Our selection of factors was based on both a scree plot of eigenvalues and Kaiser's criterion (Kaiser, 1960) with the cut-off point set at 1. Furthermore, only factor loadings greater than .4 were considered (Stevens, 1992). Of course, theoretical meaningfulness of the resulting factor structure was also taken into account.

\section{Results}

The PCA yielded three factors with eigenvalues greater than 1 (see tABLE 3.1). After rotation, both orthogonal and oblique rotations yielded a similar factor structure. For this reason, the results from the simpler orthogonal (varimax) rotation are presented here. ${ }^{2}$ The factor solution basically replicates that of Ginsburg and Karpiuk (1994; see TABLE 3.1). However, in contrast to the factor solution reported by Ginsburg and Karpiuk, we found that $\mathrm{Cn}$ did not load on the repetition factor, whereas Pk exclusively contributed to the repetition factor.

\section{Discussion}

Together with those of Ginsburg and Karpiuk (1994), our findings imply that the 9 RNG indices can be grouped into three clusters. The fact that these three factors represent orthogonal dimensions suggests that they tap different aspects of executive functioning. Repetition consists of the rehearsal of the same digit in succession, with excessive repetition being related to general deficits in suppression of previous responses (i.e., output inhibition; Bradshaw \& Mattingley, 1995). Seriation can best be understood as an inability to suppress stereotypical schemas (e.g., Williams et al., 2002), like counting forward, backward, by two's and so forth. This bias can be interpreted as the consequence of interference by overlearned tendencies to arrange numbers according to their natural order. Cycling occurs when individuals attempt to systematically use every possible alternative before repeating any digit, which means that they successfully monitor previous output (e.g., Williams et al., 2002).

\section{Study 2}

To investigate temporal stability of the RNG indices, the task was administered twice to a subsample of healthy controls and schizophrenic patients with an interval of two weeks. We hypothesized that the RNG scale would show satisfactory stability.

\section{Method}

\section{Participants}

Participants were 59 young adults (subsample of study $1 ; 17$ men) and 10 ( 8 men) of a total of 26 schizophrenic inpatients (see studies 3 and 4). Mean age was 19.27 years $(S D=1.54$; Range: $18-26)$ for the young adult sample and 37.40 years $(S D=11.81$; Range : 18-59) for the 10 patients. Mean educational level of the patients was 4.80 (SD = 1.03; anchors: 1 = lower education; 7 = university degree; Verhage, 1964). 
Schizophrenic patients were recruited from two psychiatric hospitals in Belgium. Diagnoses were based on DSM-IV (Diagnostic and Statistical Manual of Mental Disorders, $4^{\text {th }}$ ed.; American Psychiatric Association, 1994) criteria for schizophrenia and were made by a team of experienced psychiatrists who conducted structured diagnostic interviews. All patients were on fixed doses of antipsychotic medication, either typical $(88 \%)$ or atypical $(12 \%){ }^{3}$ Duration of illness of the schizophrenic sample was 5.81 years $(S D=6.21)$. None of the participants had a history of severe neurological disorders, substance abuse, or (co-morbid) DSM-IV axis 1 or 2 disorder. The study was approved by the standing ethical committee of the Faculty of Psychology, Maastricht University.

\section{Materials and Procedure}

Materials and procedures in session one (RNG1) was identical to those in Study 1. During session two (two weeks later), these samples had the RNG task administered for a second time (RNG2).

\section{Statistical analysis}

Using an alpha of .05; two-tailed, test-retest stability (using Pearson and Spearman correlations) and practice effects (paired samples $t$-tests and signed rank test) were explored using the three RNG factors established in Study $1 .{ }^{4}$ To this end, separate RNG parameters were z-transformed and factor scores were computed by averaging across relevant RNG indices.

\section{Results}

Test-retest stability and practice effect data are summarized in taBLE 3.2. In the subsample of healthy controls, we found the highest test-retest correlation for the seriation factor. In the schizophrenic subsample $(n=10)$, the highest test-retest correlation (Spearman's rho) was found for the RNG factor cycling. In the healthy as well as the schizophrenic sample, no practice effects were found for the three factor scores.

\section{Discussion}

In healthy controls, RNG factors appear to possess at best modest test-retest stability. Meanwhile, with repeated administration, healthy controls did not show significant practice effects for the three factors. For the schizophrenic sample, highest test-retest stability was found for the cycling factor, with no practice effects on the three factors. Test-retest correlations of the RNG scales in healthy controls and our clinical sample failed to reach the minimum of .80 required for a clinical psychometric instrument (Anastasi \& Urbina, 1997; see also De Zubicaray, Smith, Chalk, \& Semple, 1998; Jelicic, Henquet, Derix, \& Jolles, 2001). 


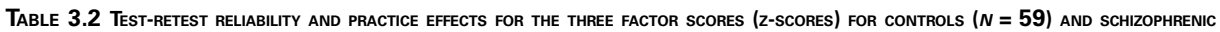
PATIENTS $(N=10)$. Means $(S D)$ are Given for Both T1 and T2.

\begin{tabular}{|c|c|c|c|c|c|}
\hline Group & RNG FACTORS & TEST-RETEST & $M(S D) \mathrm{T} 1$ & $M(S D) \mathrm{T} 2$ & $t(58)$ \\
\hline \multicolumn{6}{|c|}{ Pearson } \\
\hline Controls & Seriation Factor & $.51 * *$ & $-.23(.43)$ & $-.22(.53)$ & -.01, n.s. \\
\hline \multirow[t]{3}{*}{$\mathrm{n}=59$} & Cycling Factor & .18 & $-.05(.56)$ & $.02(.48)$ & -.62, n.s. \\
\hline & Repetitions Factor $\S$ & $.42^{* *}$ & $-.07(.73)$ & $.05(.97)$ & -1.17, n.s. \\
\hline & \multicolumn{3}{|c|}{ Spearman } & & Z statistic \# \\
\hline Schizoprenia & Seriation Factor & .43 & $1.35(1.59)$ & $1.25(1.31)$ & -.15, n.s. \\
\hline \multirow[t]{2}{*}{$\mathrm{n}=10$} & Cycling Factor & $.67^{*}$ & $.28(.40)$ & $-.11(.34)$ & -.18, n.s. \\
\hline & Repetitions Factor & .20 & $.43(1.50)$ & $-.31(.42)$ & -.38, n.s. \\
\hline
\end{tabular}

$* *=P<.001$

$\S=$ Spearman's RHO CORRELATIONS

\# WILCOXON'S SIGNED RANK TEST; (SKEWNESS REPETITION $=2.27$ )

\section{Study 3}

In this study, we investigated whether the RNG factors seriation, repetition, and cycling are related to specific neurocognitive tasks that are known to tap the constructs of inhibition of stereotypical schemas, output inhibition, and monitoring of previous output. Firstly, based on previous research (Brugger et al., 1995), we hypothesized that a failure to inhibit stereotypical schemas (i.e., heightened seriation) would positively correlate with interference susceptibility measured by the Stroop task (Stroop, 1935). Secondly, because keeping and updating information "on-line" is important for accurate monitoring of previous output and output inhibition, we expected a relationship between the central executive "online" component of working memory (backward digit span; Gerton et al., 2004), and the repetition and cycling factors. Finally, we hypothesized that the RNG factors would relate to more unitary executive function tasks in a clinical sample of schizophrenic patients (see for example Miyake et al., 2000).

A typical finding in RNG studies is that when processing demands increase (e.g., faster response pace), deviations from randomness also become more marked (e.g., Jahanshahi et al., 2006; Wagenaar, 1970). We sought to explore whether individual differences in processing speed would show a similar linear relationship with deviations from randomness. Furthermore, it has been argued that RNG is not purely driven by a limitation in non-executive working memory span (e.g., Baddeley, 1966; Wagenaar, 1970). We wanted to directly test this by relating the RNG factors to individual differences in non-executive working memory (forward digit span). 


\section{Method}

\section{Participants}

This study involved a schizophrenic subsample ( $n=26 ; 21 \mathrm{men}$ ) and a young adult subsample (see study $2 ; n=59$ ). Mean age for the schizophrenic subsample was 36.35 years ( $S D=12.83$; Range: $18-71)$. Mean educational level of the schizophrenic subsample was $4.54(S D=1.39)$. Duration of illness (in years) was $6.52(S D=7.21)$.

\section{Materials and Procedure}

Apart from the RNG task, the young adult subsample was administered the forward and backward digit span task and the Stroop colour-word test. In the schizophrenic subsample, the Behavioural Assessment of Dysexecutive Syndrome (BADS; Wilson, Evans, Emslie, Alderman, \& Burgess, 1998) and the Wisconsin Card Sorting Test (WCST; Heaton, Chelune, Talley, \& Curtiss, 1993) were administered. Schizophrenic patients were tested during standard neuropsychological screening protocols. For this reason, we did not have the opportunity to also collect the digit span and Stroop colour-word test in this sample. For the healthy sample, WCST and BADS were not administered since it is known that these instruments were designed to assess executive functions in clinical populations. Thus, these measures usually yield ceiling effects in (normal) healthy controls.

Digit span. The forward and backward digit span tests (for a full description see Stinissen, Willems, Coetsier, \& Hulsman, 1970) were administered. Each subtest was stopped after two subsequent incorrect reproductions. The number of correct orally produced strings in each subset was used as outcome measure.

Stroop colour-word test. The classic Stroop colour-word test (Stroop, 1935) was used in which participants are asked to read aloud or name the stimuli on each card (colour names of card 1, colour of the patches on card 2, and colour of the ink on card 3) one after the other as quickly as possible but without making errors. Correcting errors was allowed. However, given the infrequency of errors in this sample (mean error score $<.50)$, they were discarded in further analyses. As an index of processing speed, time to read card 2 (T2) was measured. Susceptibility to interference was calculated by subtracting $\mathrm{T} 2$ from the time needed to name the colours of the ink of card 3 (T3).

Behavioural Assessment of the Dysexecutive Syndrome (BADS). This task comprises 6 subtasks (see for a Dutch version, Krabbendam \& Kalff, 1998) and is used as a measure of executive functioning. In the current study, total profile scores (maximum $=24$ ) were used, with higher scores indicating better executive functioning. Wisconsin Card Sorting Test (WCST). A computerized version of the WCST was 
administered (128 test trials; for a full description see Heaton et al., 1993). For the present analyses, the WCST parameters "categories completed" (0-6), and "number of perseverative errors" were extracted.

\section{Results}

TABLE 3.3 shows how RNG factors relate to neurocognitive tasks. For healthy controls, a high colour naming speed was associated with a heightened seriation score. Also, a modest positive correlation was found between the RNG seriation factor and the Stroop-interference measure. For the repetition factor, a modest but significant and negative correlation was found with forward digit span. All other correlations remained non-significant. In the schizophrenia sample, the RNG factor scores of seriation and cycling correlated negatively with the BADS total score.

\section{Discussion}

In this study, we made an attempt to relate RNG factors to various neurocognitive tasks. Significant correlations were primarily found for the RNG seriation factor, albeit that these correlations were modest. Also, with so many correlations, there is the risk of experimenterwise errors. On the other hand, the significant correlations that did emerge are theoretically meaningful. For example, RNG seriation correlated positively with Stroop interference, which is not surprising when one considers that RNG seriation reflects difficulties in inhibiting stereotype responses. In this respect, our findings come close to those of Brugger et al. (1995), who reported a modest correlation ( $r=.30$ ) between Stroop interference and counting bias. We also found that a high response speed (as indexed by Stroop colour naming) is related to heightened seriation, which is not surprising if one assumes that failure to inhibit stereotypes is a trade-off of high response speed. In the schizophrenic subsample, we found significant negative correlations between RNG factors seriation and cycling and BADS scores, which is a first indication that these RNG factors are related to a more unitary executive functioning task (see Miyake et al., 2000). The significancies (i.e., $p<.01$ ) of these correlations were such that they would survive Bonferroni corrections for multiple testing.

\section{Study 4}

Several studies investigating RNG deficits in schizophrenia (e.g., Artiges et al., 2000; Horne, Evans, \& Orne, 1982; Rosenberg, Weber, Crocq, Duval, \& Macher, 1990; Salamé, Danion, Peretti, \& Cuervo, 1998; Shinba, Shinozaki, Kariya, \& Ebata, 2000) noted that schizophrenic patients have an increased tendency to produce stereotyped series and repetitive responses. Using the Ginsburg and Karpiuk factors (1994), we made an attempt to replicate this pattern. More specifically, we hypothesized that schizophrenic patients would show more extreme scores on the RNG seriation and repetition factors compared to healthy control participants (young and mid-age). 
TABLE 3.3 CORRELATIONS OF RNG FACTORS WITH STROOP COLOUR NAMING, STROOP-INTERFERENCE, AND DIGIT SPAN (FORWARD AND BACKWARD) IN HEALTHY CONTROLS $(N=59$ ) AND WITH BEHAVIOUR ASSESSMENT OF DYSEXECUTIVE SYNDROME (BADS) AND WISCONSIN CARD SORTING TEST (WCST) IN SCHIZOPHRENIC PATIENTS $(N=26)$.

\begin{tabular}{|c|c|c|c|c|}
\hline Group & TASK & SERIATION FActor & Cycling factor & REPETITION FACTOR \\
\hline \multicolumn{5}{|c|}{ Controls } \\
\hline \multirow[t]{4}{*}{$n=59$} & Stroop colour naming & $.41 * *$ & -.08 & -.11 \\
\hline & Stroop interference & $.30^{*}$ & -.02 & .07 \\
\hline & Digit span Forward & -.24 & -.03 & $-.29 *$ \\
\hline & Digit span Backward & -.20 & -.08 & -.11 \\
\hline \multicolumn{5}{|c|}{ Schizophrenia } \\
\hline \multirow[t]{3}{*}{$n=26$} & WCST number trials & -.04 & -.27 & -.03 \\
\hline & WCST pers. errors & .07 & .32 & .26 \\
\hline & BADS total score & $-.54 * *$ & $-.48 * *$ & -.32 \\
\hline
\end{tabular}

** $P<.001$

Apart from psychopathology as possible predictor of RNG performance, there is the issue of aging. Van der Linden, Beerten, and Pesenti (1998) were the first to find that elderly participants (age Range: $60-70$ ) produce on random generation tasks more series, but not more repetitions in comparison to young adults (age Range: 20-30). This is probably due to the demands that such tasks place on the central executive capacity of the elderly participants. We were interested whether a similar age-related decline in seriation factor would be found in a middle-aged group (aged 40-60) in comparison to young adults.

\section{Method}

\section{Participants}

In this study, data of studies 1-3 were collapsed and further extended with a middleage subsample. Thus, study 4 relied on the schizophrenic subsample $(n=26)$, the young adult subsample ( $n=299$; now with specific age Range: 18-25), and a middle aged subsample $(n=40 ; 17$ men; Mean age $=48.14 ; S D=8.56$; age Range: $40-62$; hereafter mid-age). Mean educational level of the mid-age sample was $5.03(S D=$ 1.05).

\section{Materials and Procedure}

Materials and procedures were identical to those used in study 1.

\section{Results}

One-way Analyses of Variance (ANOVA's) were carried out for the separate RNG factors. Apart from the mean z-transformed factor scores, mean scores on the 9 different RNG indices are also given in TABL 3.4 for normative purposes. The only effect was a significant effect of group status on the seriation factor. ${ }^{5} \mathrm{~A}$ Games-Howell post-hoc test revealed that schizophrenic patients scored higher than both young adult and the mid-age subsample $(p<.01) .{ }^{6}$ No significant differences emerged between the young adult and mid-age subsample (all $p$ 's $>.05$ ). 
Table 3.4 Normative data (means and $S D$ ) of the RNG factors (Z-transformed factor scores) and individual RNG indices (absolute SCORES) FOR YOUNG $(N=299)$ AND MID-AGE $(N=40)$ HeALTHY CONTROLS, AND THE SCHIZOPHRENIC SAMPLE $(N=26)$.

\begin{tabular}{|lcccc|}
\hline & Young & Mid-AGE & ScHizopHRENIA & $\begin{array}{c}\text { STATISTICS } \\
\boldsymbol{F}(\mathbf{2}, \mathbf{3 6 2})\end{array}$ \\
\hline Factor & Mean $(S D)$ & Mean $(S D)$ & Mean $(S D)$ & \\
\hline Seriation & & & & \\
\hline Cycling & $-.08(.69)$ & $-.03(.71)$ & $.98(1.73)$ & n.s. \\
\hline Repetition & $.00(.43)$ & $.05(.57)$ & $-.03(.34)$ & n.s. \\
\hline & $-.01(.96)$ & $.08(.75)$ & $-.06(.77)$ & \\
\hline RNG indices & & & & n.s. \\
\hline Cn & & & $18.58(6.26)$ & n.s. \\
\hline Gp & $20.87(8.39)$ & $23.21(12.26)$ & $8.33(.96)$ & n.s. \\
\hline Pk & $8.36(.68)$ & $8.31(.65)$ & $4.15(3.27)$ & $>6.0, p<.005$ \\
\hline Rn & $4.35(3.27)$ & $4.60(2.73)$ & $2.06(1.92)$ & n.s. \\
\hline Rp & $1.17(.50)$ & $1.17(.49)$ & $1.27(1.80)$ & $>6.0, p<.005$ \\
\hline Sr & $1.40(2.83)$ & $1.76(2.42)$ & $41.08(14.32)$ & n.s. \\
\hline VD & $33.62(8.27)$ & $34.17(8.49)$ & $8.79(5.85)$ & $>6.0, p<.005$ \\
\hline DR & $7.62(5.25)$ & $7.31(4.39)$ & $48.96(8.41)$ & $>6.0, p<.005$ \\
\hline Cr & $43.37(4.55)$ & $43.95(4.69)$ & $1.83(.68)$ & \\
\hline
\end{tabular}

\section{Discussion}

Criterion-based validity of the RNG task is most promising for the seriation factor, as this factor was able to differentiate between schizophrenic patients and healthy controls. This accords well with previous studies showing a strong counting bias in schizophrenic patients (e.g., Horne et al., 1982; Rosenberg et al., 1990). However, unlike these previous studies, we found no significant difference between schizophrenic patients and controls for the repetition factor. This has probably to do with the low frequency of repetition biases in our samples. Also contrary to our expectation, we did not find significant RNG differences between the young adult and mid-age healthy controls. Apparently, our mid-age subsample was too young and too healthy to find subtle deficits in central executive resources with the RNG parameters.

\section{General discussion}

This study replicated previous findings with the RNG task, but also added new data about the psychometric properties of the RNG task. More specifically, the present studies examined factor structure, test-retest reliability, construct validity, and criterion-related validity of the Ginsburg and Karpiuk (1994) RNG indices in samples of young adults, mid-age adults, and schizophrenic patients.

Our extracted factors resemble those from previous RNG factor analyses (Friedman \& Miyake, 2004; Miyake et al., 2000; Towse \& Neil, 1998) using Towse and Neil's RgCalc program indices. In previous studies, PCA identified three uncorrelated factors, with the first factor loading on randomness indices similar to our seriation factor, i.e., indices that are sensitive to the degree to which stereotype sequences are produced (prepotent associates). The second factor had high loadings for indices showing clear similarities with our cycling factor, i.e., indices assessing the degree to which each number is produced the same frequency, named "equality of response usage." Factor three was described by Friedman and Miyake (2004) as repetition avoidance, which is similar to our repetition factor. 
The test-retest correlations of the RNG scales in healthy controls and schizophrenic patients failed to reach the minimum of .80 required for a sound clinical tool (Anastasi \& Urbina, 1997). When comparing the test-retest reliability of the RNG to more traditional, well-studied executive function tasks like the WCST (see for example Heaton et al., 1993), its stability is modest. However, no substantial practice effects were found on RNG factor scores of healthy controls and schizophrenic patients. As our study was one of the first to explore test-retest stability of the RNG, future studies should further shed light on this issue, using larger samples of clinical patients and healthy participants over various periods of time (e.g., two weeks vs. six months).

As hypothesized, seriation was found to be related to processing speed and interference susceptibility in healthy controls and general executive functioning in the schizophrenic sample. In this clinical group, poor executive functioning was also associated with the cycling factor. Thus, it seems that RNG indices loading on the seriation and cycling factors measure deficits in executive or "frontal" functions, possibly originating from psychopathology or neurological deficits. For the repetition factor, floor effects may explain why this factor was not associated with other neurocognitive tasks. In both the healthy and the clinical sample, correlations between most RNG factors and various neurocognitive tasks were moderate. Future studies should relate Ginsburg and Karpiuk's factors to other neurocognitive tasks to further establish construct validity of the RNG, or conduct latent variable analyses to see whether these factors relate to a more unitary executive function or represent independent executive subprocesses (e.g., Miyake et al., 2000).

Over the past years, several cognitive and structural theoretical models for explaining RNG deviations from randomness have been introduced, such as the aleatory model (Treisman \& Faulkner, 1987), the network modulation model (Jahanshahi, Profice, Brown, Ridding, \& Rothwell, 1998), the Wagenaar model $(1970,1972)$ and the Baddeley model (1986; Baddeley et al., 1998). A detailed description of these models is beyond the scope of this chapter. However, what these models share is that they converge on the notion that RNG is attention demanding and reflects the limited capacity of central executive working memory and other executive functions (but see Treisman \& Faulkner for a signal-detection based model), needed to suppress stereotyped sequences (inhibition) and to track and update recent responses (monitoring output) (see Baddeley, 1986; Baddeley et al., 1998; Jahanshahi et al., 1998). The neural substrate underlying RNG is most likely a network encompassing primarily the left dorsolateral prefrontal cortex (e.g., Jahanshahi et al., 1998). Thus, RNG is considered to be at the controlled end of the controlled-automatic continuum (see also Jahanshahi et al., 2006). The lack of practice-related improvement between the two RNG sessions in our second study further emphasizes the key role of controlled executive functioning (see also Jahanshahi et al., 2006). The data presented in this manuscript give some tentative evidence that at least three different subfunctions contribute to RNG and that not only externally induced response pace, but also individual differ- 
ences in speed of processing affect the production of random series.

The limitations of the current studies deserve some comment. To begin with, given that our samples consisted largely of undergraduate students, most of whom were women, our samples had specific age constraints. Similarly, our studies relied on a highly specific clinical sample (i.e., schizophrenic patients), and so the usefulness of our data for normative purposes in clinical practice is limited. The effect of medication on randomization in clinical samples would also deserve further attention. Another limitation of psychometric studies like the present one is the multiple statistical testing, which raises the probability of experimenterwise errors. Where possible and appropriate, we tried to reduce that probability by applying Bonferroni corrections. Also our studies can best be seen as a first step and the next steps could involve experimental manipulation (e.g., by dual tasks) of the RNG factors and their correlates that we identified. In future research, it may also be worthwhile to determine discriminant validity pertaining to constructs such as global intelligence and simple sustained attention. A final limitation of our studies is that we employed the 1-sec condition of the RNG task, which differs from the $1.5 \mathrm{sec}$ condition in the Ginsburg and Karpiuk study (1994; but see Jahanshahi et al., 2006). Indeed, parametric research in which response pace times are systematically varied in different samples (i.e., healthy and clinical) might be informative.

Summing up, the RNG task appears to be a promising task to measure inhibition, updating, and monitoring functions in normal as well as clinical populations. Failures in these functions are reliably tapped by the RNG task. Although it does not (yet) possess the psychometric properties of a clinical tool, as a research tool the RNG may help us understand nonrandom response biases in healthy humans and even more prominent deviations from randomness in clinical populations. 

PART [B]

SOURGE MONITORING,

PSEUDO-MEMORIES,

AND NEUROBOGNITIVE

FONGTIONS IN

UNDERGRADUATES

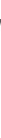





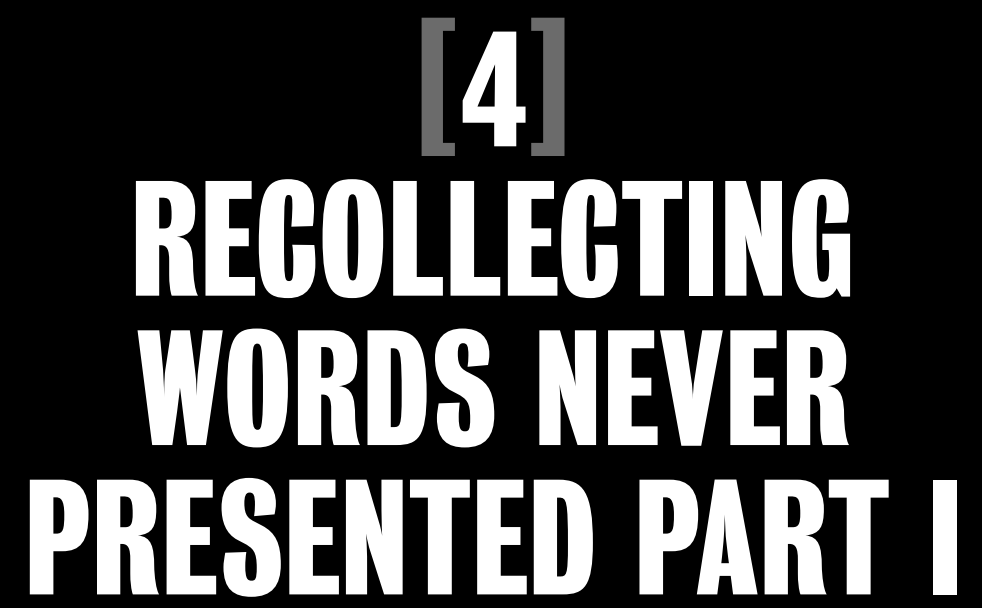

Mild executive dysfunctions in undergraduate students 



\title{
CHAPTER [4] REGOLLECTING WORDS NEVER PRESENTED PART I Mild executive dysfunctions in undergraduate students
}

An adjusted version of this chapter is published as: Peters, M.J.V., Jelicic, M., Haas, N., \& Merckelbach, H. (2006). Mild executive dysfunctions in undergraduates are related to recollecting words never presented. International Journal of Neurosciene, 116, 1065-1077.

\begin{abstract}
The aim of this study was to explore whether individual differences in executive function in undergraduate students $(n=72)$ contribute to false recall and recognition as obtained with the Deese/Roediger-McDermott (DRM) paradigm. Participants were administered the DRM paradigm and were also given a test designed to assess executive function -the Random Number Generation task (RNG). A relationship was found between heightened series on the RNG (indicating a deficiency in the ability to inhibit stereotyped cognitive schemes) and false recognition of non-presented critical lure words in the DRM paradigm. This suggests that individual differences in executive function do occur in a healthy population and that the reconstructive activity inherent to memory depends in part on executive functioning.
\end{abstract}




\section{Introduction}

Although the power of memory is evident in various daily life experiences (e.g., personal history, knowledge of facts and concepts, and learning of complex skills), memory also has its fallible side (Kopelman, 2002; Schacter, 1999). Beginning with Sir Frederic Bartlett's (1932) reconstructive viewpoint, cognitive psychologists have developed experimental paradigms to elicit or identify memory errors, some of which involve pseudo-memories (Garry, Manning, Loftus, \& Sherman, 1996; Loftus, Miller, \& Burns, 1978; Roediger \& McDermott, 1995). In this context, a widely used method is the Deese/Roediger-McDermott (DRM) paradigm, initially developed by Deese (1959b) and later modified by Roediger and McDermott (1995). As described in Chapter 1, the crux of the DRM paradigm is that people try to remember lists of related words, such as bed, nap, pillow, and snooze, all of which are associated to a common word, in this particular example the word sleep. The word sleep, however, is never presented in the study list and serves as a critical lure word at test. Following each list presentation, participants are asked to recall the studied items. Once all lists have been presented and recalled, participants are given a recognition test comprising the studied words, unrelated lures, and critical lures. Roediger and McDermott (1995) reported that on the average, participants falsely recognized 65-80 per cent of the non-presented critical lure words. These findings were replicated in a standardization study by Stadler, Roediger, and McDermott (1999) that explored which lists are most susceptible to elicit false recognition. In our Chapter 2, we presented similar results for a Dutch sample.

Following the introduction of experimental paradigms to elicit memory errors, research focused on the identification of individual differences in susceptibility to these errors. Thus, personality traits like dissociation, suggestibility, and imagery vividness (e.g., Candel, Merckelbach, \& Kuijpers, 2003; Horselenberg, Merckelbach, Muris, Rassin, Sijsenaar, \& Spaan, 2000; Winograd, Peluso, \& Glover, 1998) were studied to see whether they modulate performance on tasks that elicit memory errors. While research along these lines has yielded important clinical insights, its theoretical contribution is limited. One approach that might shed more light on this issue is to look for neurocognitive functions that may explain why certain traits are related to false recollections. Recent aging and lesion studies suggest that neuropsychological factors (in particular executive functions such as monitoring and inhibition) play an important role in the creation of false recollections (see Dodson \& Schacter, 2002a; Kopelman, 1999, 2002; Melo, Winocur, \& Moscovitch, 1999). According to Miyake, Friedman, Emerson, Witzki, and Howerter (2000) executive functions are general-purpose control mechanisms (e.g., inhibition of prepotent responses, monitioring, and updating of working memory representations) that modulate the operation of various cognitive subprocesses and its networks are primarily related to the frontal lobe. Several clinical studies have shown that impaired executive functions resulting from prefrontal lobe damage are associated with pathologically high false recogni- 
tion rates (e.g., Curran, Schacter, Norman, \& Gallucio, 1997). Likewise, functional Magnetic Resonance Imaging ( $\mathrm{fMRI}$ ) and Event Related Potential (ERP) studies relying on neurologically healthy participants indicate that recognition of critical lure words in the DRM paradigm is related to late onset activity of the prefrontal cortex (e.g., Schacter, Buckner, Koutstaal, Dale, \& Rosen, 1997).

When retrieving encoded information from memory, the prefrontal cortex is involved in retrieval search strategies and the evaluation of their retrieval results (post-retrieval monitoring; e.g., Squire \& Schacter, 2002). Its primary role is evaluating and monitoring relevant information and inhibiting irrelevant information during retrieval. The latter function can be described as cognitive inhibition, which serves to discriminate target memories from similar competing memories (inhibiting irrelevant schema information in favor of relevant schema information; e.g., Anderson \& Spellman, 1995). Thus, the question arises whether less effective cognitive inhibition could lead to an increase in false recollections during, for example, a recall or recognition phase in the DRM paradigm. After all, during this phase, a decision must be made about whether activated retrieval information reflects a veridical word recollection or whether it is an unrelated or critical lure intrusion. Ineffective cognitive inhibition might lead to liberal criterion setting (i.e., less strict in discrimination of relevant and irrelevant information), resulting in less reliable retrieval (i.e., more false recollections).

A task that taps cognitive inhibition is the Random Number Generation (RNG) task (Ginsburg \& Karpiuk, 1994). In this task, participants are asked to produce long sequences of the numbers 1-10 in a random fashion. Successful performance on the RNG requires efficient control of response generation and suppression, as people have to suppress their natural preference for counting in series (e.g., inhibition). The RNG has been effective in detecting loss of cognitive flexibility in a number of neurological diseases (e.g., Parkinson's disease: Brown, Soliveri, \& Jahanshahi, 1998; autism: Williams, Moss, Bradshaw, \& Rinehart, 2002). Several indices have been proposed to measure the various departures from randomness (Ginsburg \& Karpiuk, 1994; 1995; Chapter 3 of this dissertation). A factor analysis on RNG data revealed three clusters of random indices: repetition, seriation, and cycling related to output inhibition, inhibition of stereotyped cognitive schemes, and successful monitoring of previous output, respectively (Williams et al., 2002; see also Chapter 3). Nested under these superordinate clusters are a number of reliable RNG indices (e.g., Giesbrecht, Merckelbach, Geraerts, \& Smeets, 2004; Williams et al., 2002; Chapter 3), notably repetition and poker (factor repetition), series (factor seriation), and variance of digits (factor cycling).

As mentioned earlier, neuropsychological determinants of false recollections have only been studied in special populations, i.e., in patients with brain injury (Melo et al., 1999), older people (Lödvén, 2003), and children (Alexander, Goodman, Schaaf, Edelstein, Quas, \& Shaver, 2002). The link between executive functioning and sus- 
ceptibility to false recollections within a healthy group (e.g., undergraduate students) has received little attention. It is not too farfetched to assume that there are individual variations in executive functions in such samples. The aim of the current study was to explore whether executive function in undergraduates is linked to false recall and recognition as measured with the DRM paradigm. Because monitoring of memory retrieval does involve inhibition of competing schemata, we expected that individual differences in inhibition of stereotyped cognitive schemata assessed with the RNG would be related to false recollections in the DRM paradigm.

\section{Method}

\section{Participants}

Participants were 72 undergraduate psychology students (18 men) who agreed to take part in the study in return for course credits. Mean age of the participants was 19.10 years ( $S D=2.52$; Range: $18-33$ ).

The data obtained in this study partly overlap with another study investigating the Dutch version of the Deese/Roediger-McDermott paradigm (see Chapter 2). Nevertheless, we report the data of both studies separately because they have distinct research goals and draw upon a different body of literature.

\section{Materials and Procedure}

Deese/Roediger-McDermott Paradigm. Participants were told that we were interested in memory functioning. They were administered a Dutch version of the Deese/ Roediger-McDermott paradigm (Deese, 1959b; Roediger \& McDermott, 1995). We created 64 word lists, of which 32 were translated from Stadler and colleagues (1999), while the other 32 were based on Dutch word association norms (Lauteslager, Schaap, \& Schievels, 1986; Van Loon - Vervoorn, \& van Bekkum, 1991; van der Made-van Bekkum, 1973). Each list consisted of 15 words semantically related to a non-presented critical lure. The 64 lists were randomly divided into 4 blocks of 16 lists. Each participant was presented with one of the 4 blocks (16 lists per participant), with the 4 blocks counterbalanced across participants. The lists were read out loudly one after the other. The words (spoken by a female voice) were presented for $1 \mathrm{sec}$ with an inter-stimulus interval of $1 \mathrm{sec}$. After each list presentation, participants were given 2 min to write down all the words they could remember. Participants were given a 5 min filler (arithmetic) task after the recall of the $16^{\text {th }}$ list. They were then given an old-new recognition task consisting of 16 critical lures of the studied lists completely intermixed with 48 study words (the $1^{\text {st }}, 8^{\text {th }}$ and $10^{\text {th }}$ word of each studied list) and 32 unrelated lures taken from the list of the three non-presented blocks. Only the words that were unrelated to the words in the studied lists were used as unrelated lures. For each of these 96 words, participants had to indicate whether the word was old (i.e., had appeared on one of the 16 lists) or new. 
Random Number Generation. The DRM task was followed by the RNG task. In this task, participants were asked to generate random sequences of digits (numbers between 1 and 10, with 1 and 10 included), for a period of $100 \mathrm{sec}$, one digit each sec (rhythm was indicated by a metronome adjusted to $60 \mathrm{bpm}$ ). Participants were instructed to synchronize their responses with the pacing metronome tones, and to say out loud a number with each tone. The concept of randomness was explained using the analogy of picking numbers out of a hat. To establish an attention set for the relative timing of the pacing tones, participants were instructed to just listen to and not respond to the first few tones. Scoring of RNG was based on parameters described by Ginsbrug and Karpiuk (1994) and Williams et al. (2002). We focused on 4 indices: Repetition and poker (repetition factor), series (seriation factor), and variance of digits (cycling factor; see also Chapter 3 ).

\section{Statistical analyses}

Before collapsing the 64 word lists, data of the 32 translated word lists from Stadler and colleagues (1999) and the 32 word lists based on Dutch word association norms (Lauteslager et al., 1986; Van Loon - Vervoorn, \& van Bekkum, 1991; van der Madevan Bekkum, 1973) were compared as to their efficacy to elicit studied words and critical lure words. To this end, independent samples $t$-tests were used. Statistical analyses were carried out with alpha $=.05$.

Firstly, associations between RNG and DRM indices (i.e., recall and recognition of study words and critical lures) were explored using Pearson product-moment correlation coefficients. Next, using independent samples $t$-tests, we compared true and false recall and recognition in participants scoring either low or high on RNG (i.e., those with scores below the $25^{\text {th }}$ or above the $75^{\text {th }}$ percentile).

\section{Results}

In comparing the 32 word lists of Stadler and colleagues to the 32 word lists based on Dutch word association norms, no significant differences were found in their capacity to elicit studied words or critical lure words (all $p^{\prime} s>.05$; two-tailed). In this respect, collapsing the two types of word lists was appropriate.

Mean proportion scores on recall and recognition of study words and critical lures in the DRM paradigm are presented in TABLE 4.1. Two participants were excluded from further data analyses because of prior knowledge of the DRM procedure. The overall probability of recalling and recognizing a critical lure was .26 and .49, respectively. Mean values for repetition, poker, series and digit variance were 1.80 ( $S D=$ $2.97), 4.61(S D=3.70), 33.67(S D=7.12)$, and $7.80(S D=5.38)$, respectively. 


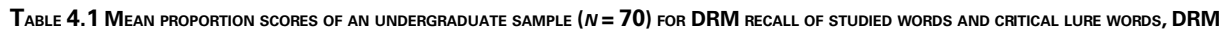
RECOGNITION OF STUDIED WORDS AND CRITICAL LURE WORDS.

\begin{tabular}{|llll|}
\hline DRM & M & SD & RANGE \\
\hline Recall studied words & .64 & .07 & $.48-.77$ \\
\hline Recognition studied words & .83 & .10 & $.40-1.00$ \\
\hline Recall critical lure & .26 & .18 & $.00-.75$ \\
\hline Recognition critical lure & .49 & .27 & $.00-.94$ \\
\hline
\end{tabular}

TABLE 4.2 presents Pearson correlations between recall and recognition of critical lures on the one, and repetition, series, variance of digits, and poker, on the other hand. No significant correlations were found between either recall or recognition of the study words and the 4 different RNG parameters (all $p$ 's > .05; two-tailed). Recognition of critical lures was significantly correlated with series scores $(r=.36, p<$ .01). ${ }^{1}$ Similarly, there was a borderline significant correlation between recall of critical lures and series $(r=.23, p=.08$; two-tailed).

When extreme groups were formed based on RNG indices (i.e., performance below the $25^{\text {th }}$ or above the $75^{\text {th }}$ percentile), no differences between these groups emerged in terms of recall or recognition of study words or critical lures (all $p$ 's > .05; two tailed). There was one interesting exception: Those scoring low on series also had low recognition rates of critical lures compared to those scoring high on series, mean recognition rates being $.37(S D=.27)$ and $.63(S D=.24) ; t(36)=3.15, p<.01)$. Likewise, participants scoring low on series had lower recall rates of critical lures relative to those scoring high on series, means being .19 ( $S D=.16)$ and $.28(S D=.16)$; $t(36)=1.70, p<.09$.

Table 4.2 Pearson correlations between DRM recall and ReCognition of Critical luRe Words and RNG INDices (REPETITION, SERIES, VARIANCE

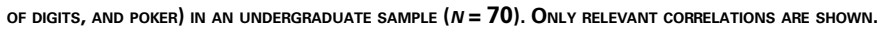

\begin{tabular}{|lcc|}
\hline & DRM RECALL & DRM RECOGNITION \\
\hline DRM Recall & & \\
\hline DRM Recognition & $.68^{*}$ & .21 \\
\hline Repetition & .11 & $.36^{*}$ \\
\hline Series & .23 & .24 \\
\hline Variance of digits & .12 & .22 \\
\hline Poker & .10 & \\
\hline
\end{tabular}

Notes: * $P \leq .01$

\section{Discussion}

Our results show that even in a healthy sample of undergraduate students, individual differences in executive functions are related to experimentally induced pseudomemories. In the extreme groups of low and high RNG series, the raised false recall and recognition rates of those producing a relatively high number of series were not accompanied by heightened levels of true recall or recognition. Thus, our findings can not be attributed to a better learning effect in the high series group. The association between RNG series and false recognition in the DRM paradigm indicates that reduced inhibition of stereotyped cognitive schemata is related to an enhanced susceptibility to false recollections. Given that undergraduates generally perform well on executive tasks, it seems likely that our participants with high RNG series scores 
exhibited only subtle executive dysfunction. This might explain why we failed to find other reliable associations between RNG parameters and false recollections in the DRM paradigm. Meanwhile, our findings are well in line with those of Alexander et al. (2002), Lödvén (2003), and Melo et al. (1999), who found associations between executive dysfunctions and false recollections in special samples. To our knowledge, the present study was the first to explore whether individual differences in executive functions within a normal undergraduate sample are related to false recollection.

According to the theoretical framework proposed by Dodson and Schacter (2002a), the prefrontal cortex is involved in updating, suppression (i.e., inhibition), and monitoring of learned information. Their Constructive Memory Framework (CMF) proposes that false recollections are modulated by neuropsychological factors operating primarily during the encoding or retrieval stages of memory. There are two problems that have to be solved in order for the memory system to reconstruct accurate memories of past events. Firstly, during encoding, features must be connected together to form a "coherent" representation (i.e., the feature binding process). It is also necessary to keep the bound representations separate from each other (pattern separation). During retrieval, the memory system must also solve binding and separation problems in order to reconstruct relatively accurate memories of past events. Secondly, once memory representations have been retrieved, the memory system faces another problem, referred to by Johnson (1992) as the source monitoring problem. This phase of post-retrieval monitoring involves criterion-setting: Determining whether the memory representation is a veridical recollection of an experienced event or a fantasy. The CMF emphasizes that the prefrontal cortex sustains both retrieval focus and criterion-setting. Plainly, both processes involve cognitive inhibition of schema-related material. In our study, this aspect was tapped by a reduced ability to suppress stereotypical series of digits (e.g., 2, 3,4). Such a lack of cognitive inhibition may lead to liberal criterion setting and an inability to suppress related information, with the potential consequence of source misattributions. In the DRM paradigm, poor cognitive inhibition may underlie the acceptance of semantically associated critical lure words and subsequent pseudo-memories.

Our failure to find a significant correlation between the DRM indices and the repetition and cycling indices of RNG might have to do with a reduced tendency of the normal group to engage in cycling and repetition (e.g., Williams et al., 2002; Brown et al., 1998). Meanwhile, a study by Brown and colleagues demonstrated that, like Parkinson patients, their normal control group showed biases in RNG, including a strong counting tendency (e.g., series) and repetition avoidance. Generation of random numbers requires the production of a novel sequence of items, as well as the inhibition of previous responses, schemas, or strategies, such as counting. Excessive repetition may indicate an inability to suppress previous responses effectively. This process of output inhibition involves suppressing the mental representation or initiation of a previous motor response. In contrast, excessive series may suggest a deficiency in the ability to inhibit a stereotyped schema (e.g., 1, 2, 3). 
In comparing the false recall and recognition rates ( $26 \%$ and $49 \%$, respectively) that we obtained to those obtained in other studies using the DRM paradigm, our rates appear to be relatively low. In the first experiment by Roediger and McDermott (1995), the non-presented associates were recalled $40 \%$ of the time. In their second experiment, false recall and recognition of the critical item were even higher $155 \%$ and $76 \%$, respectively). A possible explanation for this discrepancy could be a greater variance of our lists in probability to elicit the critical lure. In our experiment, we used 64 lists, comparing to 6 lists in the first and 24 lists in the second experiment of Roediger and McDermott (1995). When looking at our lists with the highest probability of eliciting the critical lure word, mean probabilities for recall and recognition of the critical lure word were $52 \%$ and $75 \%$, respectively.

Although the current study highlights interesting questions for future research, some limitations need to be discussed. In our study, the inhibition function was measured using the RNG task. One could argue that as a measure of inhibition, this task is of restricted value. Past studies, however, have demonstrated that poor performance on RNG is not attributable to a misconception of randomness (Baddeley, 1998; Wagenaar, 1970), or short term memory problems. Rather, using dual-task paradigms, it has been shown that this task taps the ability to inhibit prepotent responses and cognitive schemas (Towse \& Valentine, 1997; Williams et al., 2002). More specifically, Baddeley, Emslie, Kolodny, and Duncan (1998) pointed out that inhibition and switching of retrieval plans are the crucial components of RNG. The RNG has been effective in detecting loss of cognitive flexibility in a number of neurological (e.g., Parkinson's disease: Brown et al.,1998; autism: Williams et al., 2002; dementia of the Alzheimer type: Brugger, Monsch, Salmon, \& Butters, 1996), psychiatric populations (e.g., depression: Watkins \& Brown, 2002; schizophrenia: Artiges et al., 2000), and normal populations (Joppich, Däuper, Dengler, Johannes, Rodriguez-Fornells, \& Münte, 2004; Van der Linden, Beerten, \& Pesenti, 1998). The RNG therefore appears to be an appropriate measure of the inhibition function.

Another obvious limitation of the current study is its correlation approach. While our results are consistent with the notion that mild inhibitory deficits linked to frontal lobe functioning contribute to false recollections, we cannot exclude other causal interpretations. Clearly, this causal issue needs to be addressed by further studies. One way to do this is to expose healthy participants who vary in their executive efficiency to a dual task and to examine how increased cognitive load affects their susceptibility to memory errors. Another point that warrants further study is how executive efficiency is related to individual difference measures that are known to be involved in false recollections (e.g., dissociation, fantasy proneness; Winograd et al., 1998).

To sum up, a bulk of research has shown that a variety of false recollection phenomena can be observed in both patients who have suffered frontal lobe damage (see for reviews Kopelman, 1999; Parkin, 1997) and older adults (Kensinger \& 
Schacter, 1999). In this study, we found mild executive dysfunction in undergraduates to be related to remembering words that were never presented. The current findings demonstrate that it might be worthwhile to look at how neuropsychological factors may contribute to healthy people's false recollections. We used the DRM paradigm in undergraduate students, but future research may focus on generalizing these findings to other experimental paradigms (e.g., imagination inflation; Garry et al., 1996) and clinical populations with a specific deficit in inhibitory aspect of executive functioning (e.g., schizophrenia, Attention Deficit Hyperactivity Disorder). Research on this field is important because it might provide a starting point for new insights to the factors contributing to the fallibility of our memory, using performances on neuropsychological tests. 



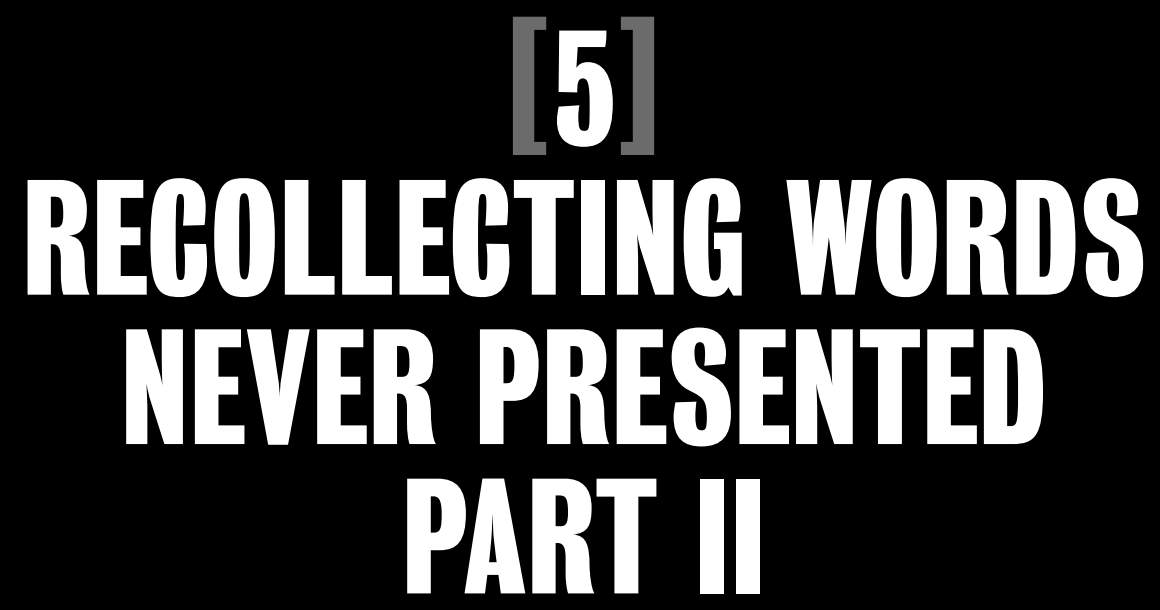

Poor working memory capacity in undergraduate students 



\title{
CHAPTER [5] RECOLLEGTING WORDS NEVER PRESENTED PART II Poor working memory capacity in undergraduate students
}

An adjusted version of this chapter is published as: Peters, M.J.V., Jelicic, M., Verbeek, H., \& Merckelbach, H. (2007). Poor working memory predicts false memories. European Journal of Cognitive Psychology, 19, 213-232.

\begin{abstract}
Two studies investigated whether individual differences in simple span verbal working memory and complex working memory capacity are related to memory accuracy and susceptibility to pseudo-memory development. In study 1 , undergraduate students $(n=60)$ were given two simple span working memory tests: Forward and backward digit span. They also underwent a memory task that is known to elicit pseudo-memories of non-presented words, the Deese/Roediger-McDermott (DRM) paradigm. Poor simple span working memory (as reflected by suboptimal backward digit span scores) was related to elevated levels of false recognition. Study 2 replicated this finding in that suboptimal backward digit span performance of undergraduates ( $n=65$ ) was found to be predictive of false recognition. However, complex working memory capacity (Operation span) was not related to false recognition. This pattern suggests that even in a homogenous sample of undergraduates, poor working memory is associated with the susceptibility to recollect words never presented.
\end{abstract}




\section{Introduction}

During memory retrieval, various encoded pieces of the memory trace need to be integrated into a reconstructive recollection of an event. This memory binding process has been the object of much research and has been ascribed to a neural network encompassing posterior brain regions, the hippocampus, and the prefrontal cortex (e.g., Kroll, Knight, Metcalfe, Wolf, \&Tulving, 1996; Moscovitch, 2000; see also Chapter 1). Apart from their role in accurate retrieval, various researchers have argued that neurocognitive functions related to this neural network (working memory, monitoring, executive control) are also involved in the creation of distortions (e.g., remembering a blue car, when the colour of the car was red) and pseudo-memories (remembering events that never took place; e.g., Dodson \& Schacter, 2002a; Gonsalves \& Paller, 2002; Kopelman 1999, 2002; Marsch, Balota, \& Roediger, 2005; Melo, Winocur, \& Moscovitch, 1999; Parkin, 1997; Schacter, 1999; Schacter \& Slotnick, 2004). One important antecedent of pseudo-memories is a breakdown in what has been termed source monitoring (Johnson, Hashtroudi, \& Lindsay, 1993). Source monitoring refers to the mechanism that allows people to determine the source of memory information. It is a mechanism that serves as a screening and controlling device for memory at retrieval, in which distinctive perceptual information plays an important role in labelling events as veridical.

The crux of working memory is the maintenance and manipulation of information, both during memory storage and retrieval from long-term store (e.g., Baddeley \& Hitch, 1974; D'Esposito \& Postle, 2002; Kane \& Engle, 2002). Working memory is closely connected to executive functioning and the prefrontal cortex (e.g., D'Esposito $\&$ Postle, 2002). There are also good reasons to believe that working memory subserves source monitoring (e.g., Hedden \& Park, 2003; Mitchell, Johnson, Raye, \& Greene, 2004; Moscovitch, 2000; Reinitz \& Hannigan, 2004). Studies that support this line of reasoning were carried out by Mitchell and colleagues (2004) and Reinitz and Hannigan (2004). Mitchell et al. (2004) conducted a study to identify the neural correlates related to maintaining memory representations active in working memory for subsequent source memory evaluations. In three functional Magnetic Resonance Imaging (fMRI) experiments, a memory paradigm was used in which participants were exposed to series of four items presented sequentially for $1 \mathrm{sec}$ each. To create source conditions, two of the items were presented as words, two were blackand-white line object drawings (format condition), and each of these item types was presented on the left or the right side of the screen (location condition). To maximize working memory involvement, participants were probed immediately after each trial (consisting of four items) to make source and familiarity judgments. The researchers found that this working memory paradigm yielded greater activation in the lateral prefrontal cortex for source memory judgments compared to familiarity judgments. Further support for the link between working memory and source monitoring comes from a study by Reinitz and Hanningan (2004). In one of their three experiments, the 
effect of working memory overload (by dividing attention during a digit monitoring task) on subsequent pseudo-memory development for compound words was investigated. The authors found that when participants had to monitor specific digits (total number of even digits) that were simultaneously presented with compound words (e.g., toothpaste, headache), they more often tended to later falsely remember never presented compound words (e.g., toothache), thus misattributing these separate words to their wrong source. Thus, it can be hypothesized that through increased susceptibility to source monitoring errors, poor working memory (either tested direct by working memory tasks or indirect by using dual-tasks) may lead to an increase in pseudo-memories.

One important mechanism to distinguish veridical from pseudo-memory traces is the amount of perceptual detail, with veridical memory traces exhibiting more of this distinctive information (Johnson et al., 1993). This issue is further supported by recent neuroimaging and electrophysiological studies that suggest that sensory activation is greater for true compared to false recognition (see for a review Schacter \& Slotnick, 2004). When making source monitoring judgments, these perceptual details serve as landmarks in evaluating a memory trace as trustworthy, i.e., veridical. A source monitoring problem may arise when during encoding of specific event information, this perceptually based information supporting source monitoring at time of retrieval is poorly encoded. This can happen when working memory capacity is overloaded, either indirectly, when situational circumstances put increasing demands on working memory (i.e., remembering telephone number and simultaneously your shopping list of tonight; dual tasks) or directly when working memory resources are poor for neurobiological reasons. Thus, reduced, overloaded or suboptimal working memory processing would lead to poor encoding of perceptually based information, resulting in a lower level of active maintenance of information (e.g., source information). This could undermine performance on cognitive challenging tasks that require such active maintenance. After all, encoding and retrieval of an accurate memory representation requires information about the source of the representation (Johnson et al., 1993; see the activation-monitoring framework for a comparable line of reasoning; McDermott \& Watson, 2001), in order to make memory attributions (i.e., "Does this memory representation originate from an event or from a dream?").

The connection between deficits in specific neurocognitive functions (working memory, executive functions) and pseudo-memories has been primarily studied in neurological patients (e.g., Melo et al., 1999), older people (Lödvén, 2003), and children (Alexander, Goodman, Schaaf, Edelstein, Quas, \& Shaver, 2002; Ruffman, Rustin, Garnham, \& Parkin, 2001). Yet, it is not unreasonable to assume that even in healthy undergraduate samples, there is individual variation in the efficacy of neurocognitive functions that might affect their susceptibility to pseudo-memories. Surprisingly, only a few studies explored individual differences in specific neuro- 
cognitive functions sustained by the prefrontal cortex and hippocampus that may contribute to pseudo-memory development. In an earlier study (see Chapter 4), we explored whether mild executive dysfunctions (closely related to working memory) in undergraduate students are linked to false recall and recognition of semantically related words. To this end, we employed the Deese/Roediger-McDermott (DRM: Deese, 1959b; Roediger \& McDermott, 1995) paradigm. Apart from this paradigm, participants in our study were given a test designed to assess executive function -the Random Number Generation task (RNG; see Chapter 3). In the study described in Chapter 4, we found that participants with high series scores on the RNG exhibited higher false recognition rates of the DRM critical lures than those with low series scores. High series scores reflect a lack of inhibition of stereotyped cognitive schemata and, thus, subtle executive dysfunctions.

A recent study by Watson, Bunting, Poole, and Conway (2005) was the first to investigate whether individual differences in complex span working memory capacity (WMC) are related to pseudo-memories for non-presented critical lure words in the DRM paradigm. In two experiments, undergraduate participants were initially screened with the Operation span task (O-span task; La Pointe \& Engle, 1990). In this task, participants are required to read aloud a math problem, followed by a to-beremembered word, e.g., "Is $(8 / 4)+5=7$ ? SEA." After several trials of these equationword pairs, participants are prompted to recall all of the words presented during the trials in the correct order. Operation span is defined as the sum of the correct recalled words across all individual trials. Based on a screening with the O-span task, 50 high and 50 low O-span participants were selected from the upper and lower quartiles of span score distribution. These participants were subjected to the DRM paradigm. Half of the high and low span participants received prior to encoding an explicit warning instruction about the potential of the DRM paradigm to elicit pseudo-memories and were encouraged to avoid recalling the critical lure word for each of the associative lists. The remaining half of high and low span participants were not given any warning (experiment 1). The results of this experiment showed that undergraduates with low WMC (low O-span) more often falsely recalled critical lure words than individuals with high WMC (high O-span), but only so when participants had received a warning about the DRM paradigm. These findings suggest that individual differences in WMC influence encoding of distinctive information, thereby affecting later post-retrieval monitoring and the ability to actively maintain task goals. In case of poor working memory capabilities, this may result in an enhanced susceptibility to pseudo-memories in young adults.

Inspired by Watson and colleagues (2005), we conducted two studies to further disentangle the relationship between source monitoring, individual differences in working memory, and pseudo-memory development in a healthy student sample. Given that (a) source monitoring is important for accurate performance on the DRM and (b) working memory plays an important role in encoding and retrieving distinc- 
tive information necessary for accurate post-retrieval monitoring, one would expect higher levels of pseudo-memories when working memory performance is poor. However, in contrast to Watson and colleagues (2005) who only used a complex working memory capacity task, we wanted to find out if this line of reasoning also holds for different measures (i.e., aspects) of working memory (simple vs. complex working memory capacity). Therefore, study 1 examined whether individual differences in simple span verbal working memory are linked to pseudo-memory development as measured by the DRM paradigm. The study by Watson and colleagues (2005) did not combine recall and recognition memory tasks, and therefore we decided to include both DRM parameters in study 1. The forward and backward digit span subtasks of the Wechsler Adult Intelligence Scale (Wechsler, 1997; for a Dutch translation, see Stinissen, Willems, Coetsier, \& Hulsman, 1970) are widely used measures of simple span verbal memory in neuropsychological research and clinical practice (see Iverson \& Tulsky, 2003 for normative data). Both tasks require immediate, serial recall of a list of digits that are read out loud and, typically, the length of the digit string is increased until the participant consistently fails. The two tasks tap different aspects of working memory (Baddeley, 1996). Of most interest in this study is the backward digit span task, because this task is believed to rely to a large extent on the central executive component of Baddeley's model (Gerton et al., 2004). The central executive serves as a controlling device for the two slave systems: phonological loop (forward digit span) and visuo-spatial sketchpad. If working memory (encoding of distinctive features) subserves source monitoring, we expect that even in a relatively homogeneous sample of undergraduates, performance on the simple span verbal working memory test (backward digit span) would be related to false recall and recognition in the DRM paradigm. We anticipated that forward digit span (non-executive) performance would not or to a lesser degree be associated with false recollections in this paradigm.

\section{Study 1}

\section{Method}

\section{Participants}

The sample consisted of 60 psychology undergraduate students (43 women) at Maastricht University. They received course credits in return for participation. Mean age of the participants was 19.6 year ( $S D=2.86$; Range: $18-38$ ). Exclusion criteria were psychiatric disorders, brain injury, or language difficulties.

\section{Procedure and materials}

The study was approved by the standing ethical committee of the Faculty of Psychology of Maastricht University. Participants were tested individually in a quiet laboratory room. Upon amival, participants were asked to sign an informed consent form. Instructions, manipulations, and stimulus materials were given on paper. 
Deese/Roediger-McDermott Paradigm. Participants were subjected to a Dutch version of the Deese/ Roediger-McDermott (DRM) paradigm (Deese, 1959b; Roediger \& McDermott, 1995). The DRM paradigm used in the current study consisted of 10 selected word lists drawn from a Dutch normative study (see Chapter 2). Each list consisted of 15 words semantically related to a non-presented critical lure word. The lists were read aloud one after the other. The words (spoken by a female voice) were presented for $1 \mathrm{sec}$ with an inter-stimulus interval of $1 \mathrm{sec}$. Unlike the study by Watson and colleagues (2005), participants did not receive a warning manipulation. After each list presentation, participants were given 2 min to write down all the words they could remember. The completion of the 10 lists was followed by the digit span task (see below). Participants were then given an old-new recognition task consisting of 10 critical lures of the studied lists completely intermixed with 30 study words (the $1^{\text {st }}, 8^{\text {th }}$ and $10^{\text {th }}$ word of each studied list) and 20 unrelated lures taken from non-presented lists. Only words unrelated to the words in the studied lists served as unrelated lures. For each of these 60 words, participants had to indicate whether the word was old (i.e., had appeared on one of the 10 lists) or new.

Forward and backward digit span. Following the DRM recall task, participants were given the digit span test to assess verbal working memory. It was taken from the Wechsler Adult Intelligence Scale (WAIS; for a Dutch translation see Stinissen et al., 1970). The test consisted of two subtests: forward and backward digit span. Strings of digits were read aloud (e.g., 24 7), each string increasing in length (from 2 digits to 8 digits). After every string, the participant was asked to repeat the string. The test consisted of 12 strings in the normal front to back order (forward) and 12 strings in the back to front order (backward). Each subtest (forward and backward) was stopped when a participant incorrectly reproduced two successive strings. The number of correctly reproduced strings was used as a measure of verbal working memory.

\section{Data analysis}

Data analyses were carried out with alpha $=.05$; two-tailed. The following six DRM indices were derived: mean proportion accurate recall, false recall of critical lure words, false recall of unrelated lures, mean proportion of correctly recognized old words, recognition of critical lure words, and recognition of unrelated lures. Because we were primarily interested in pseudo-memories, we focused on recall and recognition of critical lure words. First, Pearson correlations were calculated between forward and backward digit span and the six DRM parameters. Multiple regression analyses (Enter method) were conducted with forward and backward digit span scores as independent measures and mean proportion recall and recognition of critical lure words as dependent variables. 


\section{Results and discussion}

TABLE 5.1 summarizes data of the six DRM parameters. The overall probability that participants recalled the critical lure was $.47(S D=.19)$, while that for recognizing the critical lure was $.87(S D=.15)$. The mean proportion score for recognizing unrelated lures as old was $.03(S D=.04)$.

Table 5.1 Mean proportion scores (Including SD and range) for recall of Studied and critical LURe Words, and unRelated lures. Mean PROPORTION SCORES FOR RECOGNITION OF STUDIED WORDS, CRITICAL LURE WORDS, AND UNRELATED WORDS ARE ALSO SHOWN. FORWARD AND BACKWARD DIGIT SPAN PERFORMANCES ARE EXPRESSED AS MEAN OVERALL SCORES.

\begin{tabular}{|c|c|c|c|c|}
\hline ITEM TYPE & & $M$ & $S D$ & RANGE \\
\hline \multicolumn{5}{|l|}{ Recall } \\
\hline & Recall studied words & .61 & .07 & .39 \\
\hline & Recall critical lures & .47 & .19 & .80 \\
\hline & Recall unrelated lures* & .03 & .02 & .07 \\
\hline \multicolumn{5}{|c|}{ Recognition } \\
\hline & Recognition studied words & .79 & .11 & .47 \\
\hline & Recognition critical lures & .87 & .15 & .60 \\
\hline & Recognition unrelated lures & .03 & .04 & .20 \\
\hline \multicolumn{5}{|l|}{ Digit Span } \\
\hline & Forward digit span & 6.10 & 1.21 & 4.00 \\
\hline & Backward digit span & 4.65 & 1.05 & 5.00 \\
\hline
\end{tabular}

* EXPRESSED AS PROPORTION OF ALL RECALLED WORDS THAT WERE UNRELATED LURES [RECALL UNRELATED LURES DIVIDED BY TOTAL RECALL SCORE (SUM RECALL STUDIED WORDS, CRITICAL LURES AND UNRELATED LURES)].

Forward and backward digit span performance scores were not significantly related to each other $(r=.22, p>.05)$, showing that they tap more or less independent aspects of working memory. Pearson product-moment correlations were calculated between the six DRM indices and forward and backward digit span scores. No significant correlations were found between recall and recognition of the studied words on the one, and the digit span task, on the other hand (recall hits: $r_{\text {forward }}=.11, p=.40$; $r_{\text {backward }}=.19, p=.15$; recognition hits: $\left.r_{\text {forward }}=.09, p=.52 ; r_{\text {backward }}=.16, p=.21\right)$. Neither were there significant correlations between recall/ recognition of unrelated lures and the two digit span parameters (recall unrelated lures: $r_{\text {forward }}=.06, p=.62 ; r_{\text {backward }}=-.07$, $p=.62$; recognition unrelated lures: $\left.r_{\text {forward }}=.05, p=.70 ; r_{\text {backward }}=-.05, p=.69\right)$. However, there was a borderline significant correlation between recall of critical lures and backward digit span ( $r=-.23, p=.08$; two-tailed). Similarly, proportion recognition of critical lures was significantly and negatively correlated with backward digit span ( $r=$ $-.40, p<.01) .{ }^{1}$ Correlations between recall/ recognition of the critical lure and forward digit span remained non-significant (respectively $r=-.05, p>.05$ and $r=-.15, p>$ .05).

Two multiple linear regression analyses were carried out to determine how much variance in recall and recognition of critical lures was explained by forward and backward digit span scores. The results are shown in TABLE 5.2. Both forward and backward digit span were entered in the model (Enter method) to explain the variance in recall of the critical lure word. Neither forward nor backward digit span contributed significantly to the model. A second regression model to explain the variance in recognition of critical lures by entering both forward and backward digit span 
showed that only backward digit span accounted significantly for the tendency to falsely recognize the critical lure word as old. In this model, $16 \%$ of the variance was explained $\left(R^{2}=.16\right)$.

\begin{tabular}{|c|c|c|c|c|}
\hline VARIABLE & B & SE B & 及 & $t$ \\
\hline \multicolumn{5}{|l|}{ Recall } \\
\hline Forward digit span & 3.27 & .02 & .00 & .00 \\
\hline Backward digit span & -.04 & .02 & -.23 & -1.70 \\
\hline \multicolumn{5}{|l|}{ Recognition } \\
\hline Forward digit span & -.01 & .02 & -.06 & -.51 \\
\hline Backward digit span & -.06 & .02 & -.39 & $-3.10^{*}$ \\
\hline
\end{tabular}

NOTE. $\boldsymbol{R}^{2}=.051$ FOR RECALL; $\boldsymbol{R}^{2}=.16$ FOR RECOGNITION.

B = UNSTANDARDIZED REgRESSION COEFFICIENT; SE B = STANDARD ERROR UNSTANDARDIZED REgRESSION COEFFICIENT; $B$ = STANDARDIZED REGRESSION COEFFICIENT; $T=T$-VALUE

$* P<.01$

The main results of our study can be summarized as follows. Backward digit span was significantly and negatively related to the recognition of critical lures. That is, poor backward digit span performance was associated with heightened levels of false recognition. A similar pattern was evident for false recall of critical lures, but this effect reached only borderline significance. Thus, compared to participants with relatively high backward digit span scores, those with relatively low backward digit span scores made more critical lure intrusion errors, indicating a higher susceptibility to pseudo-memories (as measured by the DRM paradigm). In more general terms, then, our results show that even in a healthy sample of undergraduate students, individual differences in backward simple span working memory predict the occurrence of pseudo-memories. This supports the hypothesis that when working memory processing is suboptimal, it will affect encoding and later retrieval of distinctive information, such that source monitoring errors occur.

Given that undergraduates generally perform well on simple span verbal working memory tasks, lowered scores on the digit span tests in the present study should not be considered indicative of neuropsychological impairments. Mean scores on the forward and backward digit span tasks (see TABLE 5.1) are well in line with those reported by Iverson and Tulsky (2003). These authors found in their standardisation sample (aged 18-19) mean scores for forward and backward digit span of 6.7 (SD = 1.3) and $5.0(S D=1.5)$, respectively. The proportions of critical lures elicited by the DRM paradigm in the current study (being $47 \%$ and $84 \%$ for recall and recognition, respectively) were comparable to those reported by Roediger and McDermott (1995). In their first experiment, Roediger and McDermott (1995) found that the non-presented critical lures were recalled $40 \%$ of the time. In their second experiment, false recall of the critical lure was even higher (i.e., 55\%), while the false recognition rate was $76 \%$.

Our results are in line with those reported by Watson and colleagues (2005), who also failed to find a significant correlation between recall of critical lure words and a working memory task. However, contrary to these authors, we found that, even 
without warning, a negative relationship did emerge between executive simple span verbal working memory and false recognition of critical lures at testing.

\section{Study 2}

Watson and colleagues (2005) found an association between the O-span task and false recall in the DRM paradigm, but only after participants had been warned of the capability of the DRM paradigm to elicit false recall. Such a warning imposes increased demands on working memory, leading to reduced encoding of distinctive information. In our study, no warnings were given and nevertheless a correlation was found between the backward digit span task and false recognition in the DRM paradigm. There are two explanations for these discrepant findings. Firstly, reliance on different working memory tests, simple span vs. complex span, might account for the discrepancy. It could well be the case that different WMC indices (tapping different working memory loads) would have a differential effect on source monitoring. Secondly, we tested for false recognition after our participants had been given a free recall test. Thus, the recognition scores of our participants may be contaminated by prior free recall performance. To explore these two possibilities, we conducted a second study in which three different working memory measures were included: forward digit span and backward digit span as simple span tasks and O-span as a complex span task. In this second study, it was further investigated whether different working memory indices contribute differentially to source monitoring judgments. In addition, participants were given a DRM recognition task, without prior recall. We hypothesized that, since participants were not given a warning during DRM recognition, we would find no association between $\mathrm{O}$-span and false recognition. However, as in study 1 , we did expect to find a negative correlation between backward digit span and false recognition.

\section{Method}

Participants

Sixty-five (16 men) psychology undergraduate students took part in this study. They received course credits in return for participation. Mean age of the participants was 18.9 years $(S D=1.13$; Range: $17-22)$. Exclusion criteria were identical to study 1.

\section{Procedure and materials}

The procedure used was similar to that in study 1 with the exception that no free recall was obtained and that we included the O-span task. Participants were tested individually in a quiet laboratory room. After signing the informed consent form, participants underwent the DRM paradigm, the two digit span tasks, and the O-span task. 
Deese/Roediger-McDermott Paradigm. In this study, the 10 lists of the first study were extended with 6 further lists to reduce the probability of ceiling effects. The 16 word lists were drawn from a Dutch normative study (see Chapter 2). As in study 1, each list consisted of 15 words semantically related to a non-presented critical lure word. The procedure of list presentation was similar to study 1 , but this time the recall phase after each list was omitted. Lists were presented one after another with a 2 sec interlist interval. After the 16 word lists had been presented, digit span tasks and the O-span task were administered (order of working memory tasks was counterbalanced; see below). Participants were then given an old-new recognition task consisting of 16 critical lures of the studied lists completely intermixed with 48 study words (the $1^{\text {st }}, 8^{\text {th }}$ and $10^{\text {th }}$ word of each studied list) and 32 unrelated lures taken from nonpresented lists. Only words unrelated to the words in the studied lists were used as unrelated lures. For each of these 96 words, participants had to indicate whether the word was old (i.e., had appeared on one of the 16 lists) or new.

Forward and backward digit span. The digit span tasks were the same as those used in study 1.

Operation span task (O-span task). The O-span task, as described by Engle, Cantor, and Carullo (1992) and Turner and Engle (1989) is a measure of complex working memory capacity. In this task, participants are presented with operation-word pairs (i.e., operation strings). In the present experiment, the version of Engle et al. (1992) was used. The operation part is a mathematical equation which the participant has to read aloud. Next, he/she has to verify whether the proposed solution of the equation is correct or incorrect. The mathematical equation consists of 2 simple operations: a multiplication or division problem and an addition or subtraction problem. An example would be: $(8 / 4)+5=7$. Participants are not allowed to use pen and paper or to make the intermediate calculations aloud. When the participant has verified the equation, he/she has to read aloud the to-be-recalled word that stands behind the equation and press the space bar as quickly as possible. In the current study, only words that did not appear in the DRM task were used in the O-span task. Following this, another word-equation item was administered. The number of operation strings (i.e., set size) in a trial increased from two to five with three trials at each set size. Set size varied pseudorandomly. There were three practice trials each containing two operation strings and 12 experimental trials. After the last operation in a trial, the participant saw a set of three question marks centred on the screen. Participants were then asked to write down the words, in correct order, that followed the operation strings. O-span score was calculated according to the partial-credit-unit weighted (PCU) procedure as described in Conway, Kane, Bunting, Hambrick, Wilhelm, and Engle (2005). When a participant had fewer then $85 \%$ of the equation items correct, his or her O-span data were excluded. 
Data analysis

Analyses were similar to those carried out in study 1. Alpha was set at .05; two-tailed. Three DRM indices were derived: mean proportion recognition of old words, recognition of critical lure words, and recognition of unrelated lures.

\section{Results and discussion}

TABLE $\mathbf{5 . 3}$ shows the mean proportions of accurately recognized old words, falsely recognized critical lures and unrelated lures, and mean scores on digit span tasks (forward and backward) and O-span task. As can be seen, the data are well in line with the proportions found in study 1 . Thus, the hit rate for recognizing studied words was $.75(S D=.10)$, while the false alarm rate for critical lures was practically identical to the hit rate $(M=.79, S D=.18)$.

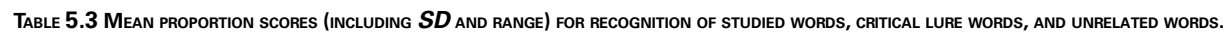
ForWARD AND BACKWARD DIGIT SPAN SCORES AND OPERATION SPAN SCORES ARE GIVEN AS MEAN OVERALL SCORES.

\begin{tabular}{|lllll|}
\hline ITEM TYPE & & M & SD & RANGE \\
\hline Recognition & & & .10 & .44 \\
\hline & Recognition studied words & .75 & .18 & .88 \\
\hline & Recognition critical lures & .79 & .12 & \\
\hline Digit Span & Recognition unrelated lures & .17 & 1.02 \\
\hline & Forward digit span & 5.50 & .81 & .09 \\
\hline & Backward digit span & 4.50 & .00 & 4.00 \\
\hline
\end{tabular}

* As indexed by partial-credit-unit weighted (PCU) score. See Conway et al. 2005)

Pearson correlations between both backward and forward digit span, on the one hand and the O-span task on the other, were non-significant (both $r$ 's $<.15$, both $p$ 's $>$ .05; two-tailed), showing that they measure different aspects of working memory. The correlation between forward and backward digit span task was significant, although its magnitude was comparable to that found in study $1(r=.27, p=.03)$. Pearson productmoment correlations were calculated between the three DRM indices, forward and backward digit span scores, and O-span scores. Replicating the findings of study 1 , no significant correlations were found between recognition of the studied words on the one, and the two digit span tasks and O-span task, on the other hand ( $r_{\text {forward }}=.02$, $p>.05$; two-tailed; $r_{\text {backward }}=-.16, p>.05$; two-tailed; $r_{\text {o-span }}=-.07, p>.05$; two-tailed). Neither were there significant correlations between recognition of unrelated lures, the two digit span parameters, and the O-span task (similar direction of relationship; all r's $<.15$, all $p$ 's $>.05$ ). However, as in study 1 , false recognition of critical lures was significantly and negatively correlated with backward digit span ( $r=-.49, p<.01$; two-tailed). Forward digit span task and the $\mathrm{O}$-span task were not significantly related to false recognition of critical lure (respectively $r=-.17, p=.18$ and $r=-.01, p=.93$ ). Figure 5.1 shows the scatterplot of backward digit span scores and false recognition of critical lures. As can be seen, the significant correlation between these two variables is not explained by outliers, an impression that is confirmed by Cook's distance (Range $=.25)^{2}$ 
As in study 1, multiple linear regression analysis was carried out to determine the amount of variance in the false recognition of critical lures explained by forward and backward digit span scores and O-span performance. The results are shown in TABLE 5.4. When entering forward and backward digit span, and O-span (Enter method) to explain variance in critical lure recognition, neither forward digit span, nor O-span contributed significantly to the model. Only backward digit span was found to contribute significantly, with the amount of variance explained by this model being 23.8 $\%\left(R^{2}=.238\right)$.

\section{Scatter Plot}

Backward digit span vs. proportion recognition critical lure

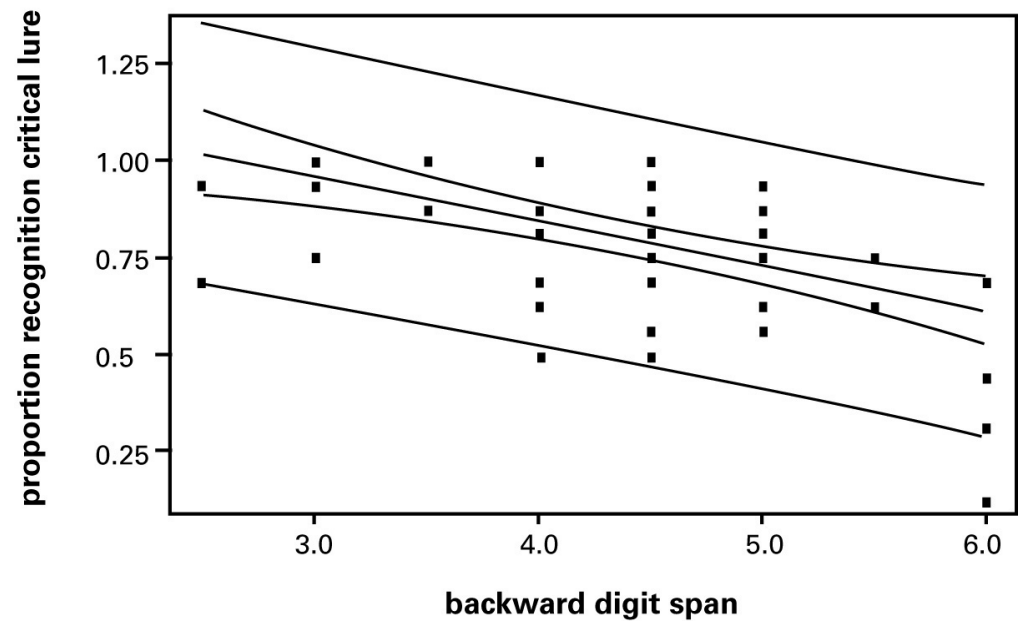

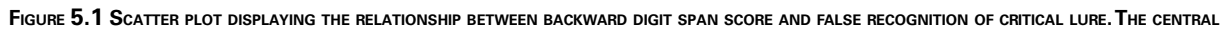
LINE DISPLAYS THE REGRESSION LINE. THE TWO OUTER LINES DISPLAY THE PREDICTION INTERVALS OF THE REgRESSION LINE FOR SINGLE OBSERVATIONS. THE TWO LINES MOST CLOSELY TO THE REGRESSION LINE DISPLAY THE PREDICTION INTERVALS OF THE MEAN PREDICTED RESPONSES.

As was the case in study 1 , we found in study 2 a negative correlation between backward digit span and false recognition of critical lure words. Replicating and extending the findings of Watson and colleagues (2005), we failed to find a link between the O-span task and false recognition. This could indicate that different working memory tasks (simple vs. complex WMC) may have a differential effect on source monitoring errors. 
TABLE 5.4 SUMmARY OF REGRESSION ANALYSIS FOR VARIABLES PREDICTING FALSE RECOGNITION OF CRITICAL LURES $(N=65)$

\begin{tabular}{|ccccc|}
\hline VARIABLE & B & SE B & B & \\
\hline Recognition & & & -.04 & -.36 \\
\hline Digit Span forward & -.01 & .02 & -.04 & $-4.12^{*}$ \\
\hline Digit Span backward & -.11 & .03 & -.48 & .20 \\
\hline Operation Span & .04 & .23 & .02 & \\
\hline NOTE, $\boldsymbol{R}^{2}=\mathbf{2 3 8}$ & & & & \\
\hline
\end{tabular}
NOTE. $\boldsymbol{R}^{2}=.238$

B = UNSTANDARDIZED REGRESSION COEFFICIENT; SE B = STANDARD ERROR UNSTANDARDIZED REGRESSION COEFFICIENT; ß = STANDARDIZED REGRESSION COEFFICIENT; $T=T$-VALUE; $* P<.01$

\section{General discussion}

In the current studies, we employed several working memory tasks to test the idea that poor working memory capacity predicts susceptibility to pseudo-memories. In the first study, the working memory tasks differed with respect to the amount of executive functioning that they required, with the backward digit span task requiring more executive functioning than the forward digit span task. In the second study, the tasks differed in terms of executive function involvement (i.e., backward versus forward digit span tasks). Yet, they also differed in terms of complexity (i.e., the number of parallel processes they required), with the O-span task tapping more processes (verbal and arithmetic) than the simple digit span tasks. The results of the two studies converge on the conclusion that, under certain circumstances, one particular type of simple span working memory, namely backward digit span, is linked to pseudomemory. Thus, our findings suggest that even in a homogeneous sample of undergraduates, small variations in working memory are linked to individual differences in pseudo-memory proneness. Reduced working memory capacity may undermine episodic encoding of the different word lists, thereby reducing the set of distinctive perceptual information needed to differentiate semantically related critical lure words from presented words. As a consequence, source monitoring errors will occur.

In general, our results are in line with those of Watson et al. (2005), but they also differ in some important respects. Like Watson and co-workers (2005), we were unable to detect a straightforward relationship between working memory capacity and false recall (study 1 ). In study 2, we extended the findings by Watson et al., in that we found no link between O-span and false recognition in a standard DRM paradigm. However, unlike Watson et al. (2005) who found a relationship between poor working memory and pseudo-memory only when participants were given a warning, we did find that the executive index of simple span working memory task predicted false recognition in a standard DRM paradigm, even when no warning instruction was given.

A question that arises is whether the discrepancy in findings between the Watson et al. (2005) study and our studies can be attributed to the use of different working memory tasks. Put in more general terms, do different working memory aspects (depending on different loading demands) differentially contribute to source monitoring? Working memory can be seen as a multi-component system that is responsible for active maintenance of information in the face of ongoing processing 
demands and/or distraction (e.g., Conway et al., 2005). Non-executive simple span tasks (i.e., tasks which only present to-be-recalled items; e.g., forward digit span) use a limited amount of resources and thus have a limited amount of loading demands, executive simple span tasks (i.e., tasks which present to-be-recalled information, but also require additional transformations; e.g., backward digit span) employ more resources (higher loading capacity), and executive-attention working memory capacity tasks (e.g., Operation span task) require a substantial amount of resources with higher loading capacity (Conway et al., 2005; Gerton et al., 2004; Oberauer, Süß, Schulze, Wilhelm \& Wittmann, 2000; Turner \& Engle, 1989). The current findings as well as those of others (Watson et al., 2005) suggest that when processing demands are high (as with warning instruction along with the DRM task in the Watson et al. study), complex working memory capacity will predict source monitoring failures. When one has poor complex WMC in a high processing demand environment, WMC is easily overloaded, leading to reduced encoding of distinctive features of the to be remembered information (e.g., semantically related words). When one has to retrieve this information, these distinctive features can not be used, leading to reliance on more general features such as semantic-relatedness, which in turn would result in source monitoring errors. On the other hand, when specific tasks do not require high processing demands (e.g., standard DRM procedures used in the present studies), simple span working memory will predict source monitoring failures. However, we do not know whether simple span working memory (non-executive and executive) is related to source monitoring failures under high processing demand circumstances (e.g., warning manipulation in the DRM paradigm). This issue warrants further research.

Several limitations of the current study deserve some comment. To begin with, our finding that working memory is negatively related to pseudo-memories was most evident for the backward version of the digit span and the recognition modality of the DRM. Note that in homogeneous samples like the present ones, strongest effects are expected to occur for the most sensitive index of pseudo-memories, namely recognition of critical lures (e.g., Roediger \& McDermott, 1995; Stadler, Roediger \& McDermott, 1999). In line with this, one has to be cautious for ceiling effects in the recognition task. Calculating corrected false recognition measure could help solving this problem. Also, the backward task is believed to be the purest measure of central executive working memory (e.g., Gerton et al., 2004). Meanwhile, the backward digit span is a subtask of the Wechsler Adult Intelligence Scale and therefore this variable overlaps with full-scale IQ. Thus, one could argue that our results reflect a link between pseudo-memory and low intelligence rather than poor working memory. However, recent research exploring the factor structure of the WAIS in a mixed patient sample and a neurological patient sample (Ryan \& Paolo, 2001; Ryan, Paolo, Miller, \& Moris, 1997) identified a distinct "working memory factor", which incorporated primarily the digit span task. It should be noted further that we tested first year psychology 
undergraduate students and thus one may assume that the full-scale IQ did not vary much in this homogeneous sample.

Secondly, one could argue that the digit span tasks we used can be considered rather crude measures of working memory. However, given the frequent use of these tests in neuropsychological research and clinical practice (see for example Gerton et al., 2004; Iverson \& Tulsky, 2003; Lezak, Howieson, \& Loring, 2004), digit span tasks have become standard measures of working memory for which clear normative data are available.

The current findings as well as those of Watson et al. (2005) provide strong support for the idea that subtle variations in executive functions and working memory of the prefrontal areas contribute to the reconstructive aspects of our memory. Our findings also accord well with previous work in our lab showing that mild executive dysfunctions in undergraduates reliably predict the extent to which they remember words never presented (Chapter 4). These findings are important because they might shed light on neuropsychological factors that make individuals susceptible to pseudo-memories. Similarly, they may help to explain why certain personality traits are intimately linked to pseudo-memories. There are a number of traits, notably dissociative tendencies and depression (or negative affectivity), which seem to predispose to pseudo-memories (e.g., Candel, Merckelbach, \& Kuijpers, 2003; Eisen \& Lynn, 2001). The connection between these traits and pseudo-memories is, however, far from robust (Horselenberg, Merckelbach, Muris, Rassin, Sijsenaar, \& Spaan, 2000). Perhaps, then, these traits serve as antecedents of pseudo-memories to the extent that they are accompanied by subtle disturbances in executive functions of the prefrontal areas. Indirect support for this line of reasoning comes from studies reporting a certain amount of overlap between poor working memory and dissociation (Giesbrecht, Merckelbach, Geraerts, \& Smeets, 2004). Clearly, the precise connection between dissociative tendencies, depression, and executive functions deserves further study. 



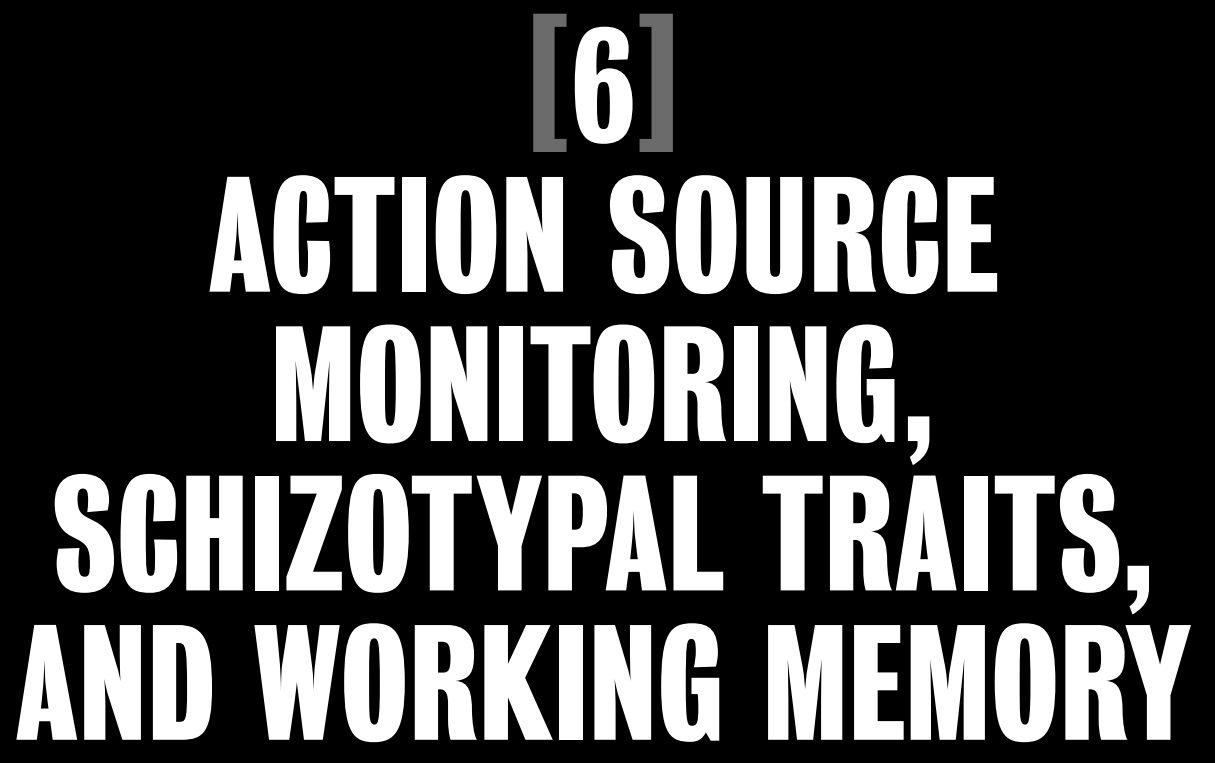





\title{
GHAPTER 6] AGTION SOURGE MONITORING, SGHIZOTYPAL TRAITS, AND WORKING MEMORY
}

An adjusted version of this chapter is accepted as: Peters, M.J.V., Smeets, T., Giesbrecht, T., Jelicic, M., \& Merckelbach, H. (accepted). Confusing action and imagination: Action source monitoring in individuals with schizotypal traits. Journal of Nervous and Mental Disease.

\begin{abstract}
To explore whether schizotypal traits may undermine source monitoring for actions, 67 undergraduate participants (21 men) completed the Schizotypal Personality Scale (STA) and were then given a source monitoring task in which some specific acts had to be performed, while others only had to be imagined. Next, participants had to complete an old-new recognition task and ascribe the source, i.e., whether they had performed or only imagined the items (i.e., source monitoring). Participants also completed a working memory capacity task (i.e., Operation Span task). We found that the higher the STA scores, the poorer recognition and source attribution scores. Relative to participants with low levels of schizotypal traits (i.e., controls), those with higher levels of schizotypal traits more often falsely claimed to have performed actions when in fact they had only imagined them. Although participants high and low in schizotypical traits did not differ in their working memory capacity, poor working memory capacity was related to source misattribution (i.e., increase false alarms). The present findings indicate that schizotypal traits undermine source monitoring for action in a healthy population.
\end{abstract}




\section{Introduction}

Most people have occasionally experienced difficulties in determining whether they have actually performed an action or only thought about performing that action in the past (Anderson, 1984; Johnson et al., 1993). However, such source monitoring difficulties (i.e., source misattributions) are especially prominent in schizophrenia (e.g., Brébion et al., 2000). Indeed, a number of recent studies have consistently shown that schizophrenic patients tend to make internal-internal source misattributions (e.g., claiming that you have said something when in fact you only thought about saying it; Nienow \& Docherty, 2004) and internal-external source misattributions (e.g., claiming that you did something when in fact someone else did it; Moritz et al., 2003). In an attempt to explain these memory aberrations in schizophrenia, Brébion et al. (2005) found that such source misattributions are related to the presence of positive symptomatology (e.g., hallucinations).

When conceptualizing schizophrenia in terms of a continuum model (Claridge, 1997), it is an interesting question to ask whether source misattributions are also present in participants with high albeit non-clinical levels of schizotypal traits. Remarkably, little is known as to whether these schizotypal traits are related to a heightened susceptibility to source misattribution. Recently, Larøi and colleagues (2005) found that non-clinical individuals with hallucination proneness (measured by the Launay Slade Hallucination Scale; Launay \& Slade, 1981) were more prone to make source misattributions. This is a first indication that these non-clinical schizotypal traits may lead to a perturbation in the control of internally generated cognitive events and thus could inform theories about how people make accurate source monitoring decisions (Johnson et al., 1993). These theories stress that encoding of perceptual features is necessary for correct source attribution at retrieval. The presence of non-clinical schizotypal traits may thus lead to problems in encoding or retrieving distinctive perceptual features of to-be-remembered events, thereby contributing to source misattributions.

A facet of memory that is related to source monitoring is working memory capacity (WMC). As said before, the source monitoring framework (Johnson et al., 1993) assumes that the encoding of perceptual details is critical for subsequent correct source monitoring decisions. Watson et al. (2005) but also our studies in Chapter 5 found that WMC is an important antecedent of the encoding of perceptual details. These studies employed the Deese/Roediger-McDermott paradigm (DRM; Deese, 1959; Roediger \& McDermott, 1995). In this paradigm, participants are asked to remember lists of semantically related words, such as bed, nap, pillow, and snooze, all of which are associated with a theme word, in this particular example the word sleep. This theme word is never presented and serves as a critical lure during recall/recognition. Both Watson et al. (2005) and our studies in Chapter 5 found that undergraduates with low WMC were more prone to make source misattributions as measured by an increased tendency to recall or recognize non-presented critical lure words. 
Since prominent WMC deficits in all modalities have been documented in schizophrenia (see for a recent meta-analysis Lee \& Park, 2005) and clinical high-risk populations (i.e., prodromal schizophrenia; Lencz et al., 2006), an interesting question to raise is whether poor WMC is also present in healthy participants with high levels of schizotypal traits and whether this may also be related to source misattributions.

Many source monitoring studies have relied on word list paradigms (e.g., DRM paradigm; cf. supra). Some have argued that the generalizibility to real life situations of such paradigms is limited (Henquet et al., 2005; Parks, 1997). Recent studies by, for example, Hornstein and Mulligan (2004) and Larøi et al. (2005) offer more naturalistic source monitoring paradigms. In these studies, simple actions were either performed or imagined, followed by a source monitoring task.

The aims of the present study were twofold. Firstly, we wanted to examine whether source misattributions are related to schizotypal traits in a non-clinical sample. To this end, we employed an action source monitoring task based on an adapted procedure described by Parks (1997) in which simple actions either had to be performed or imagined. We hypothesised that participants with high levels of schizotypal traits would make more source misattributions compared to those low in schizotypal traits. A second aim was to explore whether poor WMC could account for source misattributions in participants with schizotypal traits.

\section{Method}

Participants

Sixty-seven undergraduate students (21 men) from Maastricht University and Hogeschool Zuyd volunteered to participate in the study. No incentive was offered for participation. Exclusion criteria were diagnosis of a psychiatric disorder in the past three years, history of neurological deficit (e.g., traumatic brain injury), and substance abuse. Two participants were excluded; one participant did not complete the schizotypal trait questionnaire, while the other participant did not understand the source monitoring task. The final sample consisted of 65 participants. Their mean age was 21.01 years $(S D=2.08)$, with no significant differences between male and female participants $[t(63)=1.31, p>.05]$, means being $21.55(S D=2.26)$ and $20.81(S D=$ 1.98), respectively. The study was approved by the standing ethical committee of the Faculty of Psychology of Maastricht University.

\section{Materials and procedure}

Participants were tested individually in a quiet laboratory room. Upon arrival, participants were asked to sign an informed consent form. Instructions, description of action items, and stimulus materials were given on paper (questionnaire and recognition task) or on a computer screen (action source monitoring task and Operation span task). 
The study involved 2 sessions, with approximately $24 \mathrm{hrs}$ between sessions. All participants were naïve as to the purpose of the study. The study was presented as one in a series of studies investigating the relationship between cognitive functions, personality characteristics, and learning of simple motor actions. During the first session, participants completed the Schizotypal Personality Scale (STA; Claridge $\&$ Broks, 1984) and were administered a working memory capacity task (Operation span task; Engle et al., 1992; Turner \& Engle, 1989). Participants were also given the study phase of the action source monitoring task. Tasks were counterbalanced to exclude order effects. The second session consisted of a surprise recognition task that took place $24 \mathrm{hrs}$ after the initial study phase.

Schizotypal Personality Scale. The STA questionnaire (Claridge \& Broks, 1984; Cronbach's $\alpha=.86$ ) is designed to measure schizotypal traits in normal (healthy) populations. The STA consists of 37 dichotomous items that are closely related to the DSM-III-R criteria description of schizotypal personality disorder. This scale has been found to load on the "positive" symptoms factor of schizotypy, a factor that primarily consists of unusual perceptual experiences and psychotic-like ideation (Bentall et al., 1989; Rawlings et al., 2001). A sample item is "Are you sure that other people can tell what you think?" Total STA score is obtained by summing up "Yes" answers across all items. High scores indicate higher frequencies of non-clinical schizotypal traits (positive symptomatology).

Operation span task (O-span). The O-span task (Engle et al., 1992; Turner \& Engle, 1989 ) is a measure of complex working memory capacity. The present study employed Engle and co-workers' (1992) version. During this task, participants are presented with operation-word pairs (i.e., operation strings). The operation part is a mathematical equation that the participant has to read aloud. The mathematical equation consists of 2 simple operations: a multiplication or division problem and an addition or subtraction problem. An example would be: $(8 / 4)+5=7$. Next, he/she has to verify whether the solution that is offered for the equation is correct or incorrect. Participants are not allowed to use pencil or paper or to make the intermediate calculations aloud. When the participant has given an answer to the equation, he/she has to read aloud the to-be-recalled word that is shown immediately after the equation and to press the space bar as quickly as possible. Following this, another operation string appears. The number of operation strings (set size) within a trial increases from two to five. Every set size is employed thrice. Set size is varied pseudorandomly. Three practice trials are presented, each containing two operation strings. The O-span task consists of 12 trials. At the end of each trial, the participant is presented with three question marks centred on the screen. They are then asked to write down in correct order the words that followed the operation strings.

O-span score was calculated according to the partial-credit-unit (PCU) 
weighted procedure as described in Conway et al. (2005). If accuracy for the processing component (mathematical equation) of the task fell below a certain level (i.e., if participant had fewer then $85 \%$ of the equation items correct), his or her O-span data were excluded (see Conway et al., 2005).

Action source monitoring task. The action source monitoring task was based on a procedure described by Parks (1997) and replicated by Henquet and co-workers (2005) in schizophrenic patients. In one of Parks' studies, a card depicting questions (e.g., "When were you born?") was shown to participants for a period of $5 \mathrm{sec}$, followed by a blank card for $2 \mathrm{sec}$. After this card, participants received the instruction "Answer out loud" on it or a card that gave the following phrase in the series. Participants were instructed to read each card in silence and to be prepared to say the answer out loud without actually verbalizing the words unless they were specifically told to do so. After the study phase and a 5 min filler task, a forced-choice recognition task was presented. This task consisted of the original questions, each being paired with a new question with similar content, for which participant had to make old-new discriminations and source attributions (i.e., imagined or spoken). In the current paradigm, we replaced the question phrases with descriptions of simple actions that either had to be performed or imagined to be performed. The action items described simple non-intrusive acts like for example "Break a toothpick into three pieces" and "Open a newspaper."The items were derived from previous experiments (Goff \& Roediger, 1998; Hornstein \& Mulligan, 2004; Larøi et al., 2005). Action items were presented on a 15 inch computer screen using PowerPoint (Microsoft Corporation) with font type “Times New Roman", font size 36.

The action source monitoring task involved 40 trials. On half of them, single actions were presented. On the other half, two actions were presented, with one action being located at the top half and the other at the bottom of the screen. The order of single and dual presentation modes was quasi-random and two counterbalanced versions were used, to which participants were randomly allocated. After each action presentation, participants had to imagine performing the action(s) presented on the screen. Preparation time varied between participants but never took longer than $8 \mathrm{sec}$. When the participant indicated that he/she had imagined the action, a blank screen appeared for $3 \mathrm{sec}$. Next, an instruction appeared on the screen indicating "Do" for single action presentations and "Do top" or "Do bottom" for dual action presentations. Thus, for dual action trials participants had to imagine both actions, but actually perform only one. This resulted in 40 performed actions and 20 covertly prepared but non-performed actions.

In case certain objects or materials were needed to perform the actions (e.g., toothpick, paper, etc.), the experimenter provided participants with them after the imagination period. On the dual action trials, materials to carry out both actions were given. On dual action trials, half of the actions that had to be performed were pre- 
sented at the top, and the other half at the bottom of the screen. Immediately after the action had been completed, all objects were removed from view. Objects were hidden from the participants' view at all times, except when in use. An experimenter was present to monitor whether participants actually performed the actions. All participants were capable of performing the actions. At the end of the study phase, participants were asked to indicate how easy-difficult it was for them overall to create a mental image for the presented actions based on a 5-point scale, (anchors: $1=$ very easy to imagine; 5 = very difficult to imagine).

Recognition session. During the recognition task, the 60 old action items were paired with 60 new action items that were roughly similar in content and form. For example: "Break the toothpick in three pieces" was paired with "Break the toothpick in two pieces". For each pair, the participant was asked to make an old-new discrimination (i.e., correct recognition). Furthermore, when participants classified an action item as old, they had to indicate whether they had performed the actions or only thought about performing these actions (i.e., source attribution).

\section{Statistical analyses}

For all analyses alpha was set at .05. Proportion of correct recognition (old-new discrimination) was calculated by dividing the number of correctly identified old items in the recognition test by 60 , the total number of old items. Proportion correct source attribution was defined as the number of old items that participants correctly classified as verbalized or covertly prepared divided by 60 , the total number of source attributions that had to be made. Moreover, we calculated proportion false alarms (i.e., number of erroneous claims of imagined actions that had been performed divided by 20 , which is the total number of imagined actions) and proportion misses (i.e., number of erroneous claims that performed actions had only been imagined divided by 40 , which is the total number of performed actions).

In order to explore the relationships between schizotypal traits, action source monitoring, and WMC, Pearson product-moment correlations (two-tailed) were calculated between STA scores, action source monitoring parameters (i.e., proportion correct recognition, source attribution, false alarms and misses), and O-span task. Furthermore, following Larøi et al. (2005), participants were grouped according to their STA scores, selecting those participants with the $25 \%$ highest $(n=17)$ and $25 \%$ lowest $(n=17)$ STA scores. Independent samples $t$-tests were carried out to determine whether these groups differed in action source monitoring parameters and WMC. 


\section{Results}

\section{Ease of imagination}

Participants rated the imagination difficulty on a 5-point scale. The mean difficulty score was $1.70(S D=.74)$, which indicates that it was relative easy to imagine the action items. Thus, participants did not have any difficulties in preparing the actions.

\section{STA and O-span scores}

Mean scores on the STA and O-span task were 10.11 ( $S D=6.09$; Range: $1-23$ ) and .81 $(S D=.08$; Range: $.67-1.00)$, respectively. These scores are similar to those reported elsewhere (e.g., Engle et al., 1992; Muris \& Merckelbach, 2003) for undergraduate students. Distribution of STA and O-span scores showed no marked deviation from normality (Skewness $=.45$ and .59 , respectively). Men and women did not differ with regard to mean STA and O-span scores; both $t$ 's $(63)<1.0$, both $p^{\prime}$ s $>.05$.

\section{Correct recognition of actions}

Mean proportion of correct recognition was .89 ( $S D=.07$; Range: .53-.98). As table 6.1 shows, a significant negative correlation emerged between STA and correct recognition scores $(r=-.27, p<.05)$, indicating that those with heightened STA scores had poorer memory function. For WMC, correlations with STA and correct recognition remained non-significant.

\section{Action source monitoring}

Overall, participants were quite accurate in their source attributions ( $M=.81, S D=$ .09; Range: .45-.97). Mean proportion false alarms and misses were .18 ( $S D=.07$; Range: .10-.40) and .09 (SD = .06; Range: .00-.38), respectively. As taBLE 6.1 shows, STA scores were negatively related to correct source attribution $(r=-.36, p<.01)$, indicating that the higher participants scored on STA, the lower their number of correct source monitoring decisions. Conversely, a positive relationship emerged between STA and false alarms $(r=.27, p<.05)$. As to the WMC, a significant negative correlation was found between $\mathrm{O}$-span scores and false alarms $(r=-.30, p<.05)$. All other correlations remained non-significant.

\section{Extreme groups and source monitoring}

Two extreme subgroups were formed on the basis of their STA scores (see Larøi et al., 2005). The subgroup high in schizotypical traits consisted of 17 participants in the top $25^{\text {th }}$ percentile (STA score $>16$ ), while the control group (i.e., low in schizotypal traits) consisted of 17 participants who scored in the lower $25^{\text {th }}$ percentile (STA score $<5)$. Participant characteristics for both groups can be found in tABLE 6.2. As can be seen, participants in both groups did not differ with regard to age, gender distribution, and O-span score. 


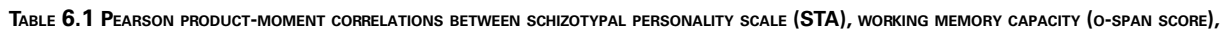
CORRECT RECOGNITION, CORRECT SOURCE ATTRIBUTION, FALSE ALARMS, AND MISSES OF THE ACTION SOURCE MONITORING TASK. ONLY RELEVANT CORRELATIONS ARE SHOWN.

\begin{tabular}{|c|c|c|}
\hline & STA & O-SPAN \# \\
\hline O-span & -.09 & --- \\
\hline Correct recognition & $-.27^{*}$ & .09 \\
\hline Correct source attribution & $-.36^{* *}$ & .14 \\
\hline False alarms & $.27^{*}$ & $-.30^{*}$ \\
\hline Misses & .17 & -.01 \\
\hline
\end{tabular}

* $P<05$

** $P<.01$

\# As indexed by partial-credit-unit weighted (PCU) score. See Conway et al., 2005)

As can also be seen in table 6.2, control participants outperformed those high in schizotypal traits on correct recognition and correct source attribution; both t's (32) $>2.60$, both $p^{\prime} s \leq .01$. Thus, participants scoring high on STA were poorer in remembering which actions they had seen $24 \mathrm{hrs}$ earlier and they were also impaired in ascribing the correct source for these actions, compared to participants with low STA scores. The effect sizes for these differences (in terms of Cohen's $d$ ) were in the large to very-large effect size Range (both $d$ 's > .90). In addition, participants high in schizotypical traits made more misses and false alarms; both $t$ 's $(32)>2.00$, both $p$ 's $<.05$. Again, effect sizes were in the large-effect Range (both $d$ 's $>.78$ ).

Table 6.2 Demographic, StA, and memory data of participants high and low in schizotypal traits. Standard deviations are given in PARENTHESES.

\begin{tabular}{|c|c|c|c|}
\hline & $\begin{array}{c}\text { PARTICIPANTS HIGH IN } \\
\text { SCHIZOTYPAL TRAITS }(n=17)\end{array}$ & $\begin{array}{c}\text { CONTROL PARTICIPANTS } \\
(n=17)\end{array}$ & Statistics \\
\hline Age & $21.41(1.58)$ & $21.12(2.23)$ & $t(32)=.44$, n.s. \\
\hline Gender (men/women) & $5 / 12$ & $6 / 11$ & $\chi^{2}(1)=.13$, n.s. \\
\hline Average STA score & $18.53(2.53)$ & $3.18(1.42)$ & $t(32)=21.82, p<.001$ \\
\hline Average O-span score & $.80(.09)$ & $.81(.05)$ & $t(32)=.20$, n.s. \\
\hline Correct recognition & $.87(.06)$ & $.92(.05)$ & $t(32)=2.60, p=.01$ \\
\hline Correct source attribution & $.77(.09)$ & $.86(.06)$ & $t(32)=3.46, p<.01$ \\
\hline False alarms & $.21(.07)$ & $.16(.06)$ & $t(32)=2.08, p<.05$ \\
\hline Misses & $.10(.06)$ & $.06(.04)$ & $t(32)=2.20, p<.05$ \\
\hline
\end{tabular}

\section{Discussion}

The main results of the present study can be summarized as follows. Firstly, significant negative correlations were found between STA scores, correct recognition, and source attribution scores, indicating poorer memory functioning and more source misattributions (i.e., false alarms) with increasing levels of schizotypal traits in healthy undergraduates. Secondly, this conclusion is further substantiated by extreme group analyses based on STA scores. These analyses showed that participants high in schizotypal traits performed poorly in correctly recognizing previously presented actions (see also Laws \& Bhatt, 2005). Furthermore, in line with prior research (Larøi et al., 2005), compared to controls, high STA participants made more erroneous claims of imagined actions as performed and vice versa. This shows that high levels of schizotypal traits are not only associated with poor recognition memory, 
but also with a profound tendency to make source misattributions. On the other hand, participants with high and low levels of schizotypal traits did not differ in their scores on the working memory indices. Meanwhile, working memory was negatively related to source misattributions, in that low WMC was accompanied by an increase in false alarms (i.e., claiming that one performed an action when, in fact, it was only imagined).

We found that participants high in schizotypal traits exhibited a deficiency in internal source attribution (e.g., "Did I do this or did I only imagine this?"), which is in line with the results of Larøi and co-workers (2005), who found that hallucinationprone (i.e., non-clinical positive symptomatology) participants made significantly more internal source misattributions. Moreover, we also showed that high schizotypal and control participants did not differ in their working memory performance. In line with these findings, Lenzenweger and Gold (2000) also failed to find specific working memory disruptions in schizotypal trait individuals. How can these results best be explained? One important prerequisite for efficient source monitoring is the encoding and/or retrieval of a sufficient amount of perceptual detail and contextual information (Johnson et al., 1993). It is plausible to assume that carrying out an action offers more perceptual, sensory, and spatiotemporal information than just imagining actions. Thus, in the former case, the production of a distinct memory trace is more probable. Perhaps, then, specific cognitive and/or personality factors (but not working memory) contribute to difficulties in encoding and retrieving such action information in participants with high levels of schizotypal traits, thereby making the memory traces of these actions less distinctive which, in turn, could lead to source misattributions.

During encoding, perceptual and contextual features of an experience (e.g., performing a specific action) need to be bound together efficiently. WMC is a necessary function for holding these memory representations online, updating, assigning memory for temporal order, manipulating information, and selective attending to-be-stored information during encoding. In the present study, we found a significant negative association between working memory and false alarms. These findings underscore previous findings of Watson and co-workers (2005) and our studies in Chapter 5 showing a robust relationship between (poor) working memory capacity and (poor) source monitoring performance. One explanation for the intimate link between working memory and source monitoring is that poor working memory restricts the encoding of distinctive information. This, in turn, may interfere with source monitoring during retrieval.

During retrieval, the encoded memory representations need to be retrieved and source monitoring decisions must be made to discriminate between previously executed actions and imagined actions. Therefore, inhibition of irrelevant information (e.g., fantasizing about imagined actions) is needed. Interestingly, previous studies have found that participants with high levels of schizotypal traits show dif- 
ficulties in actively inhibiting irrelevant information (e.g., Moritz \& Mass, 1997). Thus, participants high in schizotypical traits may be inundated with an increased array of percepts or "loose" associative (i.e., fantasizing) links. Failing inhibition then leads to increased reliance on these general similarities or fantasies, which may provide optimal conditions for source misattributions to arise. More specifically, a source misattribution and in particular a tendency to treat imagined events as having a real/ performed origin (i.e., false alarm) may convey a sense of realness to unusual sensations, paranoid ideation, and idiosyncratic beliefs and perceptions. In this way, source misattributions arise between internal experiences that are tagged with "nonself" characteristics. This line of reasoning may also apply to explaining paranormal experiences which are often reported in relation to schizotypal traits (e.g., Goulding, 2004; Lange \& Houran, 1998).

Another related possibility is that, compared to others, individuals with high levels of schizotypal traits deeply encode the characteristics of imagined actions, which could also lead to source misattributions. One argument in favour of this possibility is that schizotypy and fantasy proneness overlap substantially (e.g., $r=.60$; Merckelbach, Rassin, \& Muris, 2000). Fantasy prone individuals tend to have very intense and detailed imaginations, and this can contribute to source misattributions.

Our findings may have relevance to schizophrenia spectrum disorders. However, caution must be exercised when linking the present results to, for example, people with schizotypal personality disorder (SPD), prodomal individuals, or schizophrenic patients. That is, relations between schizotypal traits, SPD, prodrome, and schizophrenia are complex and findings are mixed in the literature. Thus, cognitive impairments in people with non-clinical schizotypal traits are very different from those in schizophrenic patients with distinct aetiologies and prodrome states, as was the case for working memory capacity difficulties in our study (e.g., Lee \& Park, 2005; Lencz et al., 2006; Lenzenweger \& Gold, 2000). Furthermore, the participants in our study were not prodromal individuals nor did they show clinical symptomatology. As it stands, the connection between schizotypal traits and schizophrenia spectrum states is not fully understood. Future research could shed light on this relationship, thereby including larger and more diverse samples for a direct comparison between the groups in question.

In sum, then, our data as well as those of others (e.g., Larøi et al., 2005) clearly imply that people high in schizotypal traits have profound source monitoring difficulties, but why this is the case remains unclear. Future studies should focus on whether encoding deficiencies, inhibition difficulties, or intense imagination related to fantasy proneness underlies this phenomenon. One limitation of the current study is that it relied on a homogeneous sample of undergraduates. Another limitation is that it did not include further personality measures or cognitive indicators such as fantasy proneness, thought action fusion, inhibition, etc. Follow up studies relying on larger heterogeneous samples and fantasy proneness and inhibition measures would be 
able to disentangle the antecedents of source misattributions in participants with high levels of schizotypal traits.

In conclusion, the present study found evidence of poor correct recognition and source monitoring in individuals high in schizotypal traits. Our results are, however, silent about the causal status of source monitoring, which can best be established in future longitudinal studies. 



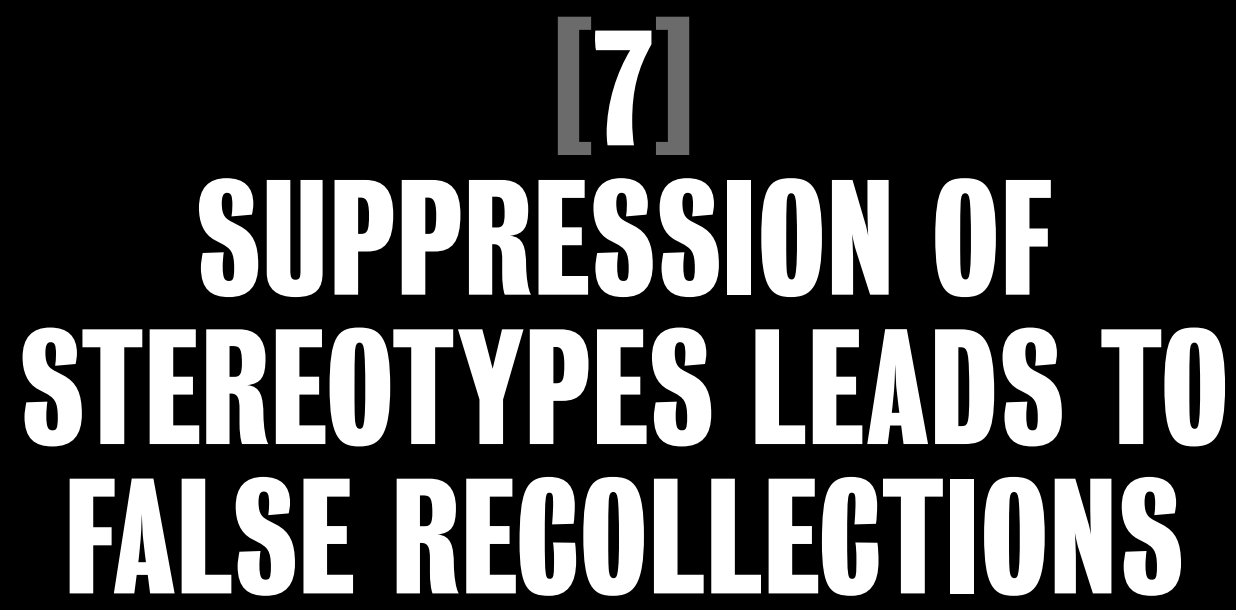

Influence of inhibition instruction on memory accuracy 



\title{
GHAPTER [7] SUPPRESSION OF STEREOTYPES LEADS TO FALSE RECOLLECTIONS

\author{
Influence of inhibition instruction on memory accuracy
}

An adjusted version of this chapter is published as: Peters, M.J.V., Jelicic, M., \& Merckelbach, H. (2006). When stereotypes backfire:Trying to suppress stereotypes produces false recollections of a crime. Legal and Criminological Psychology, 11, 327-336.

\begin{abstract}
This study examined whether participants' memories of a racially neutral crime story are influenced by stereotypes and the instruction to suppress (i.e., inhibit) stereotypes while reading the crime story. We expected that participants who saw a photograph of a foreign group (negative stereotype prime) and who were given the instruction to suppress stereotypes before reading a crime story would make significantly more stereotype-consistent errors on a recognition test than participants who received a neutral prime and a suppression instruction. Participants were 88 undergraduate students (59 women) who were randomly allocated to the cells of a 2 (negative stereotype vs. neutral prime) $\times 2$ (thought suppression vs. control) between-subjects design. The dependent variables were recognition of accurate items, stereotype-consistent items, and confabulation items. The critical stereotype $\mathrm{x}$ suppression interaction was statistically significant for false recognition of non-presented stereotypeconsistent items. Simple effect analyses showed that in the suppression condition, participants who were primed with a negative stereotype made more stereotypeconsistent recognition errors than those who had been exposed to a neutral prime. Stereotypes not only make cognitive processing easier, but might also contribute to recognition errors when people do what they often are told to do in the legal arena: suppress stereotypical thinking.
\end{abstract}




\section{Introduction}

A stereotype is a widely held belief about a certain social group (Graham \& Lowery, 2004; Hamilton \& Sherman, 1994). Different theories, ranging from sociobiological to cultural accounts (e.g., for an overview see Hinton, 2000; Schneider, 2004), have been proposed to understand how stereotypes are formed and why we use them. It appears that our tendency to rely on stereotypes is highly useful. In the words of Schneider (2004; p. 563): "We use them all the time. To deny ourselves the use of generalizations about people would result in intellectual and social chaos."

Although stereotypes may be helpful in processing complex information (e.g., Macrae, Milne, \& Bodenhausen, 1994a), an extensive body of research has shown that under some circumstances, stereotypes lead to negative evaluation and discriminatory judgments about outgroup members (e.g., Graham \& Lowery, 2004; Van Knippenberg, Dijksterhuis, \& Vermeulen, 1999). A similar ambiguity is seen when it comes to the influence of stereotypes on memory. Stereotypes facilitate encoding and retrieval of stereotype-consistent information, but they may also lead people to remember stereotype-consistent details that were never present (Araya, Ekehammar, \& Akrami, 2003; Koriat, Goldsmith \& Pansky, 2000; Lenton, Blair, \& Hastie, 2001; Macrae, Bodenhausen, Milne, \& Ford, 1997). False stereotype-consistent information is especially likely to be reported when people are instructed to deliberately suppress stereotypes. Germane to this issue is a study by Araya and colleagues (2003). These authors employed the Deese/Roediger-McDermott paradigm (DRM; Deese, 1959b; Roediger \& McDermott, 1995). Briefly, in the DRM paradigm participants are presented with a list of semantically related words (e.g., pin, sharp, point), all associated with a non-presented critical lure word (e.g., needle). The typical outcome during a subsequent memory test is that participants more often falsely recall and recognize the critical lure than non-critical lures that were never presented in the study phase (e.g., car). Araya and colleagues (2003) had their participants study stereotypical and non-stereotypical words, either with an initially activated social category (immigrant prime) or with no social category (neutral prime). Participants were then instructed to either forget or remember the studied words. This was followed by a recognition test. Participants in the immigrant, but not in the neutral prime condition more often falsely recognized non-studied stereotypical words, but only after they had previously been given instructions to forget them (see also Lenton et al., 2001). This finding fits nicely with studies demonstrating that attempts to suppress unwanted stereotypical thoughts ironically result in an increased accessibility of the unwanted thoughts (Macrae, Bodenhausen, Milne, \& Jetten, 1994b; Macrae et al., 1997; but see also Monteith, Ashburn-Nardo, Voils, \& Czopp, 2002; Monteith, Sherman, \& Devine, 1998a; Monteith, Spicer, \& Tooman, 1998b). For example, Macrae et al. (1994b) showed their participants a photograph of a male skinhead. Next, participants were asked to describe how a typical day of this individual might look like. Half of the participants were given the instruction to actively suppress/avoid think- 
ing about stereotypical ideas about skinheads while engaging in the task. The other half did not receive this instruction. Following this, participants were told they were going to meet the skinhead. While awaiting the arrival of the skinhead, they were asked to take a seat. Participants had the opportunity to choose between 7 seats. The 8th seat was evidently occupied by the skinhead, as a jacket and bag were on it. The two dependent measures were the frequency of stereotypes in the description of a typical day of the skinhead and preferred seating position. Participants in the suppression condition described less stereotypes in a typical day of the skinhead than did controls. However, suppression participants preferred to sit further away from the skinhead's personal belongings than control participants. Thus, this study found evidence for a backfire or rebound effect. That is, compared to control condition, suppression leads to an enhanced accessibility of stereotypes (see also Cook, Arndt, \& Lieberman, 2004). It should be noted here that recent research by Monteith et al. (1998b; 2002) has identified individual differences such as level of prejudice of participants, feelings of guilt, and motivation that may affect activation and application of stereotypes. These individual difference factors appear to moderate stereotype rebound effects after suppression. Yet, by and large, this research domain has yielded results that underline the paradoxical effects of suppression first described by Wegner, Schneider, Carter, and White (1987; see for a recent review, Wenzlaff \& Wegner, 2000).

The literature on suppression and its rebound effects bear relevance to the legal arena. So far, researchers have mainly looked at how mock jurors weight evidence that they were told to disregard. Again, the evidence suggests that such inadmissible evidence becomes hyperaccessible (Kassin \& Sukel, 1997). Nonetheless, many other questions remain open to discussion. For example, trying to suppress stereotypical information makes this information hyperaccessible to most persons, but what are the consequences of this for eyewitness memory? One possibility is that suppression and subsequent enhancement of stereotypical information leads to stereotypicalconsistent errors in memory (Macrae, Schloerscheidt, Bodenhausen, \& Milne, 2002). In the current experiment, we sought to examine whether an instruction to suppress stereotypes while reading a racially neutral crime story leads to pseudo-memories of and confabulations about non-presented, stereotypical information. Inspired by the Araya et al. study (2003), we predicted that participants who are primed with a foreign group photograph (negative stereotype) and who are instructed not to think of stereotypes while reading a crime story will produce more pseudo-memories of stereotype-consistent information on a delayed forced-choice recognition task, relative to participants who are primed with a photograph of typical Dutch people and who are given the instruction to suppress stereotypes. 


\section{Method}

\section{Participants}

Participants were 88 undergraduate students (59 women) from Maastricht University. Fifty-six were psychology students; the others were enrolled at other faculties. All participants had a Dutch/ European background and mastered the Dutch language. Participants received course credit for participation. Mean age of the participants was $21.3(S D=2.25$; Range: $17-27)$. The experiment was approved by the standing ethical committee of the Faculty of Psychology, Maastricht University.

\section{Design}

Participants were randomly assigned to the cells of a 2 (negative stereotype vs. neutral prime) $\times 2$ (thought suppression vs. control) between-subjects design. Scores on a recognition task served as dependent variable. There were three types of recognition items: accurate items (information in the crime story), stereotype-consistent items (information not in the crime story, but consistent with the triggered racial stereotype), and confabulation items (information not in the crime story).

\section{Procedure and materials}

Participants were tested individually in a quiet laboratory room. Upon arrival, participants were asked to sign an informed consent form. Instructions, manipulations, and stimulus materials were given in writing. Students were told that they would first participate in an unrelated experiment on facial assessment. To activate a negative stereotype, half of the participants were primed with a photograph of a group of immigrants (i.e., foreign group photograph). This photograph showed a group with a Surinamese background (4 faces of coloured individuals, 3 men and 1 woman) interacting with each other at a party. As an aside, we note that in Dutch society, Surinamese people are viewed as a well-integrated minority group. The other half of the participants was exposed to a neutral prime consisting of a photograph of typical Dutch youngsters ( 2 men and 4 women). Photographs were presented in colour $(10$ by $10 \mathrm{~cm}$ ) on a white background and were shown for $1 \mathrm{~min}$. To cover up the true purpose of the photographs, participants were asked to rate each face on the photograph on a sympathy scale ranging from 1 (not at all friendly) to 8 (very friendly). The mean sympathy scores for each picture (averaging the individual face scores) were written down and used as a manipulation check.

Thought suppression procedure. After the priming, participants in both priming conditions were assigned to a thought suppression or control (i.e., no thought suppression) condition. Before reading the crime story (see below), participants in the thought suppression condition were instructed not to think of specific stereotypes such as race, age, and gender (following Wegner et al.'s thought suppression procedure; 1987). Control participants were merely told that they were going to read a story about a crime. 
Crime story. The story was a media article about a group of young people engaging in pointless violence against a victim. Race or ethnic background of offenders and victim were left unstated. The story was presented on paper (double spaced, consisting of 694 words, comprising two pages). Based on average reading time in a pilot study $(n=6)$, time given to read the story was fixed ( $3.5 \mathrm{~min})$.

Distraction task. After reading the story, participants were given a brief distraction task consisting of two pairs of highly similar pictures that were presented to each participant. Each pair of pictures differed on 10 critical points. Participants were told to look for 10 differences between each pair of pictures within 5 min.

Forced-choice recognition task. The distraction task was followed by a forced-choice recognition task about the crime story. Participants were given the instruction to think about all the details presented in the story (including gender, race, age, etc.) and to be as accurate as possible. Participants were asked to make old-new discriminations for 60 statements about the crime story, by indicating yes (present in the story) or no (not present in the story). The recognition test consisted of 20 accurate items, 20 racial stereotype-consistent items, and 20 confabulation items. Accurate items referred to details that were present in the crime story (e.g., "The victim was last seen on Sunday the $18^{\text {th }}$ of January"). Stereotype-consistent items pertained to details not present in the crime story, but that were consistent with racial stereotypes triggered by the photograph (e.g., "The main suspect was of foreign origin"). Confabulation items were items not present in the story and that were unrelated to stereotypes (e.g., "The left hand of the suspect was swollen"). The order of item presentation was counterbalanced across participants. After finishing the forced-choice recognition task, participants were thanked for their participation and debriefed.

\section{Statistical analysis}

Three parameters were derived from the forced-choice recognition performance: Proportion correct recognition, proportion stereotype-consistent pseudo-memories, and proportion confabulations. These three parameters were subjected to a 2 (stereotype vs. neutral prime) $\times 2$ (suppression vs. control) Analysis of Variance (ANOVA). We were particularly interested in a potential interaction effect of stereotype priming and suppression on recognition of false stereotype-consistent items. Such an interaction would reflect a backfire effect.

\section{Results}

\section{Manipulation check}

Neither age nor education was statistically significant related to recognition outcome measures; all $r$ 's <.20, p's > .05; two-tailed. To examine whether the two prime conditions did, indeed, elicit specific stereotypes, we compared mean sympathy scores 
for faces in both conditions. The mean sympathy score for faces in the Dutch group photograph was significantly higher than that for faces in the foreign group photograph $[t(86)=4.97, p<.01]$, means being $5.9(S D=.86)$ and $5.1(S D=.44)$. Men and women did not differ with regard to their mean sympathy scores. This was true when sympathy data of both prime conditions were collapsed, $[t(86)<1.0]$, but also when Dutch or foreign photograph conditions were considered separately; both $t$ 's $(42)<$ 1.0 , both $p$ 's $>.05$. This indicates that our manipulation of eliciting specific stereotypes was successful.

\section{Relations among recognition measures}

We computed Pearson product-moment correlations between the three recognition parameters for the entire sample. Proportion accurate recognition was not related to proportion of stereotype-consistent pseudo-memories or proportion of confabulation (both $r$ 's $<0.15$, both $p$ 's $<.30$ ). However, we did find a positive correlation ( $r=$ $.65, p<.01$; two-tailed) between stereotype-consistent false recognition and confabulation.

\section{Forced-choice recognition}

The proportion of accurate, stereotype-consistent, and confabulation responses are shown in table 7.1. A 2 (priming) $\times 2$ (thought suppression) ANOVA performed on recognition of accurate items did not reveal significant main effects of priming $[F(1,84)$ $=1.83, p=.18]$ or thought suppression; $F(1,84)<1.0$. Neither was there a significant interaction effect; $F(1,84)<1.0$.

When recognition of false stereotype-consistent items was subjected to an ANOVA, no main effects of priming $[F(1,84)=1.74, p=.19]$ or thought suppression $[F$ $(1,84)<1.0$ ] were found. However, the critical interaction effect of priming $x$ thought suppression did emerge; $F(1,84)=5.57, p=.02$. Simple effects analysis showed that in the suppression condition, participants who had been primed with a foreign group photograph made more stereotype-consistent recognition errors $(M=.25)$ than participants who had seen a neutral prime $(M=.14) ; F(1,42)=5.35, p=.03, d=.74$. In the control (i.e., no suppression) condition, participants who had been primed with a foreign group photograph or a neutral photograph did not differ from each other with regard to proportion stereotype-consistent recognition errors, means being .18 and $.21 ; F(1,42)<1.0$. Although participants who had been primed with a foreign group photograph and subsequently engaged in suppression made more stereotype-consistent recognition errors $(M=.25)$ than non-suppressing participants $(M=.18)$, this difference fell short of significance; $F(1,42)=1.84, p=.17, d=.42$.

While an ANOVA performed on confabulatory responses revealed no main effect of priming $[F(1,84)<1.0]$ or thought suppression $[F(1,84)<1.0]$, a marginally significant interaction effect was found for endorsement of confabulatory items; $F(1$, 84) $=3.59, p=.06$. 
table 7.1 Means and standard deViations on measures PRoportion ReCognition of accurate items, false STEREOTYPE-CONSISTENT ITEMS, AND CONFABULATION ITEMS IN THE THOUGHT SUPPRESSION AND THE CONTROL (I.E., NO THOUGHT SUPPRESSION) CONDITION $(N=88)$.

\begin{tabular}{|c|c|c|c|c|}
\hline & STEREOTYPE PRIME & & NEUTRAL PRIME & \\
\hline & $M$ & $S D$ & $M$ & SD \\
\hline \multicolumn{5}{|l|}{ Thought suppression } \\
\hline Accurate & .85 & .16 & .79 & .16 \\
\hline Stereotype & .25 & .19 & .14 & .09 \\
\hline Confabulation & .21 & .17 & .14 & .13 \\
\hline \multicolumn{5}{|l|}{ No thought suppression } \\
\hline Accurate & .81 & .10 & .80 & .14 \\
\hline Stereotype & .18 & .10 & .21 & .13 \\
\hline Confabulation & .16 & .13 & .20 & .14 \\
\hline
\end{tabular}

Simple effects analyses indicated that in the suppression condition, participants who had been primed with a foreign group photograph tended to make more confabulation errors relative to participants who had been exposed to a neutral prime; $F(1,42)=2.66, p=.10, d=.46$. All other comparisons fell short of significance; $F(1,42)<1.0$.

\section{Discussion}

Early December 2004, the Dutch police showed an en face portrait of Mohamed B. on prime time television. A few weeks earlier, Mohamed B. had been arrested for killing the Dutch film director and writer Theo van Gogh. Immediately after the murder, an intense, public debate ensued in which opinion leaders warned each other for prejudices and stereotypes. It was in this context that the police showed the portrait of Mohamed B. and asked eyewitnesses to inform the police on his social network and background. Our findings suggest that this was a risky procedure as suppression of stereotypes (e.g., about Muslims) may facilitate false recognition of stereotype-consistent details (e.g., involvement in terrorism networks).

To be sure, in the psychological literature, backfire effects of instruction to suppress or avoid certain information (e.g., inadmissible evidence) have been described extensively (e.g., Cook et al., 2004; Kassin \& Sukel, 1997; Wenzlaff \& Wegner, 2000). However, relatively little is known about backfire effects on memory. We hypothesized that priming participants with a foreign group photograph and asking them to suppress their thoughts about stereotypes while reading a crime story would lead to overendorsement of non-presented, stereotype-consistent information in a subsequent forced-choice recognition task. Our results demonstrate that when given instructions to suppress stereotypes, participants who have been primed with a foreign group photograph produce more stereotype-consistent errors on a subsequent recognition task than do participants who have been primed with a neutral photograph. Furthermore and somewhat to our surprise, we found that in the suppression condition, participants who had been exposed to a foreign group photograph tended to make more confabulation errors in comparison to participants who had been given a neutral prime. This latter finding is of interest, as it suggests that participants who have been engaged in thought suppression of stereotypes not only show elevated expec- 
tancy-consistent false recognitions (i.e., more stereotype-consistent errors; see for similar findings Macrae et al., 2002; Woll \& Graesser, 1982), but also tend to accept other types of non-presented details. Accordingly, the overall correlation between stereotype-consistent and confabulation errors was significant. Further research is needed to replicate and clarify the nature of this correlation. One interpretation that suggests itself is that this correlation reflects liberal criterion setting during source monitoring activities (e.g., Johnson, Hashtroudi, \& Lindsay, 1993). Thus, it may well be the case that the combination of stereotypes and suppression encourages liberal criterion setting (e.g., Macrae et al., 2002). Source misattributions of schematic (i.e., stereotypical) knowledge may be a mechanism through which stereotypes about particular individuals or groups may be maintained and retrieved. In particular, when people receive the instruction to inhibit stereotypes, active inhibition (i.e., cognitive control) may lead to a decrease in focus on the source of information during encoding due to limited cognitive capacities (i.e., increased cognitive load). During retrieval, one may be more likely to rely on primed (negative stereotype) schematic knowledge. The thought suppression instruction can be seen as an active inhibition instruction. For thought suppression instructions, recent research has identified the prefrontal cortex as neural correlate in a network involved in inhibition. In a recent fMRI study, Wyland, Kelley, Macrae, Gordon and Heatherton (2003) found that suppression of a particular thought or all conscious thoughts, when compared to the free-thought control condition, was accompanied by increased activation of the neural network of the anterior cingulate and the insula of the prefrontal cortex. A central function of the anterior cingulate is conflict monitoring in cognitive control; inhibiting to be suppressed information (Wyland et al., 2003).

Our findings are well in line with previous work by Araya and colleagues (2003), Kimball and Bjork (2002), and Lenton and colleagues (2001). These authors also noted that pseudo-memories are fostered by forget/suppression instructions. In line with the findings of Araya et al. (2003), we found that in the suppression condition (cf. forget condition in the Araya et al. study), participants primed with a stereotype but not participants primed with neutral material falsely recognized stereotype-consistent items. And like the Araya et al. (2003) study, we found that in the control (i.e., non-suppression) condition, participants primed with stereotypes or neutral material did not differ with regard to false recognition proportions. Another issue that is worthy of note is our failure to obtain an overall (i.e., main) effect of suppression instructions. Thus, it was not the case that suppression instructions produce heightened levels of recognition errors, irrespective of primes. One could argue that this is inconsistent with the so-called rebound effect (Wegner et al., 1987; Wenzlaff \& Wegner, 2000). However, previous studies have described rebound effects primarily in terms of accessibility (e.g., Wegner et al., 1987) or overt behaviour (Macrae et al., 1994b), while work on suppression and memory has been scarce. The current findings suggest that it is the combination of suppression and stereotypes rather than 
either suppression or stereotypes alone that produces memory errors that one could interpret as a rebound.

In this study, significant differences were found between conditions in the recognition of non-presented stereotype-consistent items. However, the proportion of falsely recognized stereotype-consistent items remained relatively low. This indicates that our results were probably not due to a lenient response criterion when participants encountered stereotype-consistent items during the recognition task (Macrae et al., 2002). If this had been the case, the proportion of falsely recognized stereotypeconsistent items would have been substantially higher.

Several limitations of the current study deserve some comment. One limitation concerns the triggering of stereotypes. The manipulations we used might not have triggered stereotypes in all participants. On the other hand, there is reason to believe that overall, our manipulation did evoke such a stereotype. Germane to this is that faces in the foreign group photograph were rated as significantly less friendly than those in the Dutch group photograph.

One could argue that in our study, the unequal men-women distribution in the photographs that served as primes is a confounder. The foreign group prime showed a group of immigrants of whom $3 / 4$ were men. The neutral prime showed a group of typical Dutch youngsters of whom 2/3 were women. Since we only obtained mean sympathy scores, the possibility that the primes elicited stereotypes about men and women rather than immigrant stereotypes cannot be excluded. However, we did not find significant differences in sympathy ratings that men and women gave to the photographs, showing that both men and women evaluated the primes in a similar way. Related to this is the fact that the forced-choice recognition task included stereotype-consistent items that referred to immigrants rather than gender issues. To further clarify this important point, we conducted a pilot study in which we edited the primes using Adobe ${ }^{\circledR}$ Photoshop 6.0 so as to obtain a men/women ratio of $2 / 1$ for both of the priming photographs. If gender rather than immigrant stereotypes would drive sympathy scores, one would expect to find no differences between these two primes. We gave these 2 critical as well as 3 filler primes to 20 students ( 5 men) and asked them for sympathy scores. ${ }^{1}$ A paired samples $t$-test revealed that the mean sympathy rating given to the foreign prime was significantly lower than that given to the Dutch prime; $t(19)=6.48, p<.001$.

In this study, we used photographs of a foreign group of youngsters and a Dutch group of youngsters. One could argue that the foreign group, like the skinhead used in the Macrae et al. (1994b) study, is an example of a group for which there are few cultural and personal prohibitions against stereotyping. Thus, participants may not be motivated to suppress their stereotypes effectively (e.g., Monteith et al., $1998 a, b)$. We find this line of argumentation in the case of our primes not very convincing. In the Netherlands, the opposition against a multicultural society is not very strong (See European Monitoring Centre on Racism and Xenophobia, 2005). Also, 
there are many young foreign Surinamese (i.e., ex-colonial) immigrants in the Netherlands, even in the upper working class. Thus, a widespread and overt acceptance of stereotypes about this group is not very likely.

As mentioned before, recent work by Monteith and colleagues (e.g., 1998a, b; 2002) shows that not everyone is susceptible to the same degree of stereotype activation, suppression, and subsequent backfire effects. In a series of experiments, these researchers demonstrated that level of prejudice, motivation to control stereotyping, amount of experience or practice with stereotype control, replacing thoughts, reliance on individuating information, and the extent of automatic stereotype activation could moderate the degree to which stereotype suppression leads to subsequent backfire effects. For example, Monteith and colleagues (1998b) found that relative to participants with strong prejudices, participants with low prejudice attitudes towards homosexuals were not prone to rebound effects in terms of stereotype use and stereotype activation. In our experiment, we did not include such individual differences measures. With the results of Monteith and co-workers (1998a, b; 2000) in mind, one could expect to find the highest level of stereotype-consistent memory errors in a high prejudice group, since this group cannot exert control over their stereotypes. In future research on stereotypes and pseudo-memories, it would be worthwhile to address this issue.

To what extent can we trust eyewitnesses in courtroom settings when negative stereotypes are likely to be triggered? Our findings suggest that exposure to a stereotype (e.g., seeing a non-Caucasian suspect in the courtroom) combined with the instruction not to think of stereotypical information (e.g., disregarding evidence concerning race) might undermine memory accuracy. Stereotypes not only make cognitive processing easier, but might also lead to the creation of entirely new (and pseudo-) memories. This especially seems to be the case when people are asked to suppress stereotypically related information. 



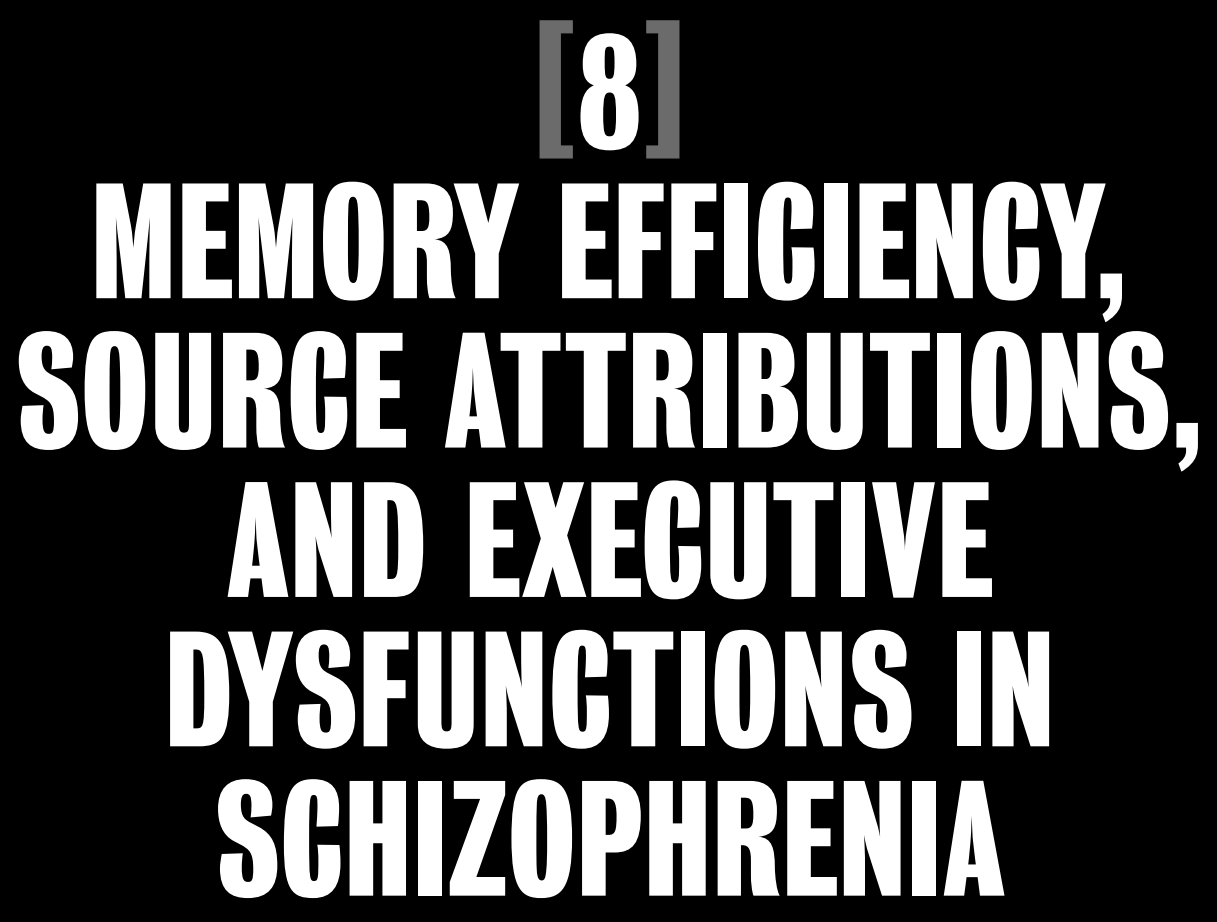





\title{
GHAPTER [8] MEMORY EFFIGIENGY, SOURGE ATTRIBUTIONS, AND EXECUTIVE DYSFUNGTIONS IN SGHIZOPHRENIA
}

An adjusted version of this chapter is accepted, pending revision as: Peters, M.J.V., Cima, M., Smeets, T., Vos de M., Jelicic, M., \& Merckelbach, H. (pending revision). Did I say that word or did you? Executive dysfunctions in schizophrenic patients affect memory efficiency, but not source attributions.

\begin{abstract}
Schizophrenic patients have difficulties in recognizing previously presented verbal information and identifying its sources. The antecedents of these recognition and source misattributions are, however, largely unknown. The current study examined to what extent schizophrenic patients' lack of memory efficiency, their memory errors, and their source misattributions are related to neurocognitive deficits (i.e., executive dysfunctions). Twenty-three schizophrenic patients and 20 healthy controls were administered an adapted version of the Deese/Roediger-McDermott (DRM) task from which parameters of memory efficiency, memory errors, source misattributions, and two-high threshold measures were derived. Furthermore, two neurocognitive tasks tapping executive functions were administered: the Wisconsin Card Sorting Test (WCST) and the Behavioural Assessment of the Dysexecutive Syndrome (BADS). Using multiple linear regression analyses, we examined whether these neurocognitive measures predicted various memory parameters. Patients with schizophrenia showed poorer memory efficiency and were more prone to make internal-external source misattributions with high confidence. However, they did not more often falsely recognize critical lure words than controls. Executive dysfunctions predicted memory efficiency, but not source misattribution performance. Our findings provide further evidence that schizophrenic patients' memory impairments are intimately related to fundamental neurocognitive deficits.
\end{abstract}




\section{Introduction}

Schizophrenia is known for its prominent cognitive deficits (Heinrichs \& Zakzanis, 1998). While memory performance of schizophrenic patients exhibits a wide range of impairments (Aleman, Hijman, de Haan, \& Kahn, 1999; McKenna, Ornstein, \& Baddeley, 2002), it is especially characterized by omission errors (i.e., misses). That is, schizophrenic patients have a tendency to omit or miss information when asked to recall or recognize specific verbal or nonverbal material (e.g., McKenna, Tamlyn, Lund, Mortimer, Hammond, \& Baddeley, 1990; Moritz, Woodward, Cuttler, Whitman, \&Watson, 2004; see Aleman et al., 1999, for a meta-analysis). However, because such omission errors can be observed in many psychiatric patients, recent research has tried to pinpoint memory aberrations that are more specific for schizophrenia (Aleman et al., 1999; McKenna, McKay, \& Laws, 2000). One memory aspect that seems to be markedly impaired in schizophrenia is source monitoring (Johnson, Hashtroudi, \& Lindsay, 1993). This refers to the ability to identify the source of remembered information, i.e., source attribution. Schizophrenic patients consistently show a deficiency in internal source attribution (e.g., "Did I do this or did I only imagine this?" Nienow \& Docherty, 2004) and reality monitoring (e.g., "Did I say this or did someone else say this to me?" Brébion, Amador, David, Malaspina, Sharif, \& Gorman, 2000; Brébion, Gorman, Amador, Malaspina, \& Sharif, 2002; Keefe, Arnold, Bayen, \& Harvey, 1999; Moritz, Woodward, \& Ruff, 2003; Vinogradov, Willis-Shore, Poole, Marten, Ober, \& Shenaut, 1997). Moritz and colleagues (2003) reported that, apart from their tendency to make source misattributions, schizophrenic patients also show exaggerated confidence in their source judgment capabilities.

Interestingly, recent studies (Elvevåg, Fisher, Weickert, Weinberger, \& Goldberg, 2004; Moritz et al., 2004; Weiss, Dodson, Goff, Schacter, \& Heckers, 2002) noted that schizophrenic patients' memory is superior to that of control individuals in one particular respect: Schizophrenic patients are less susceptible to experimentally induced pseudo-memories (i.e., false alarms of critical lures). For example, using the standard Deese/Roediger-McDermott paradigm (DRM; Deese, 1959; Roediger \& McDermott, 1995) to elicit such false alarms, Moritz and co-workers (2004) found that compared to healthy controls, patients with schizophrenia produce relatively few false alarms when confronted with semantically related lures in a recognition task (e.g., they less often falsely recognize the never presented word sleep among a list of previously presented semantic associates like bed, rest, tired, and so on). However, in accordance with other studies, Moritz and co-workers also reported that schizophrenic patients tend to make many misses on the DRM task, while showing an increased confidence in these errors.

How can this pattern of memory aberrations typical for schizophrenia best be explained? A plethora of research has demonstrated a specific link between clinical symptomatology (positive and negative symptoms) and susceptibility to source misattributions (e.g., Brébion et al., 2000; Brébion et al., 2002; Brébion, Gorman, 
Malaspina, \& Amador, 2005; see also Nienow \& Docherty, 2004, for similar findings). Moreover, recent studies demonstrated that source misattribution performance is partly dependent on intellectual ability (Vinogradov et al., 1997) and cognitive functions (e.g., verbal memory performance; Elvevåg et al., 2004; Moritz et al., 2003; Moritz et al., 2004). In an attempt to integrate these findings, Brébion and colleagues (2005) investigated the relationships between verbal memory impairments, source misattribution, and automated cognitive functions, on the one hand, and clinical symptomatology, on the other. The authors looked at two memory systems: memory efficiency and memory errors. Memory efficiency refers to the number of correct responses and can be further subdivided into superficial and deep memory processing. Brébion and co-workers found that processing speed and selective attention serve as the primary antecedents of memory efficiency. Memory errors (false alarms to critical lures or new words) were found to be associated with positive and negative symptoms. These authors also noted that source misattributions affected memory errors directly and indirectly via positive symptomatology.

There are good reasons to believe that distinct neuropsychological deficits related to schizophrenia also affect memory efficiency and memory errors (see Chapter 1 for a review). Indeed, Brébion and colleagues (2005) argued that as memory deficits (e.g., omission errors) observed in schizophrenia resemble those seen in patients with damage to the medial temporal lobe, schizophrenic patients have difficulties with deep memory processing during encoding (e.g., inefficient memory strategy). Alternatively, memory deficits in schizophrenia may be caused by executive dysfunctions. After all, an important aspect of executive functioning, which is subserved by a neural network encompassing primarily the prefrontal cortex, is monitoring the retrieval of encoded events in memory (e.g., Dodson \& Schacter, 2002; Johnson et al., 1993; Chapter 1). Because schizophrenic patients exhibit marked deficits on both executive and memory tasks (see McKenna et al., 2002; Weinberger et al., 2001), executive dysfunctions may explain memory impairments in schizophrenia over and above the contribution of symptomatology and medial temporal lobe dysfunctions (see Moritz et al., 2003; Vinogradov et al., 1997).

Most of the studies cited above looked at isolated memory dysfunctions in schizophrenic patients. That is, they specifically focused on schizophrenic patients' memory efficiency, memory errors or source misattributions. The current study relied on a paradigm that allowed examining all these different memory dysfunctions simultaneously. We also explored how certain neurocognitive impairments are related to these dysfunctions because this may inform theories about the origins of schizophrenic patients' memory aberrations. More specifically, we explored whether executive control and working memory measured by theWisconsin Card Sorting Test (Heaton, Chelune, Talley, Kay, \& Curtiss, 1993) and planning of behaviour indexed by the Behavioural Assessment of the Dysexecutive Syndrome (Wilson, Alderman, Bur gess, Emslie, \& Evans, 1996) predict memory efficiency, memory errors, and source misattributions in schizophrenic patients and healthy controls. 
A shortcoming of many previous studies in this domain (Moritz \& Woodward, 2002; Moritz et al., 2003, but see Moritz et al., 2004) is that they elicited low levels of experimentally induced pseudo-memories (i.e., false alarms). Hence, a laboratory task was employed that is known to elicit high levels of pseudo-memories, even in healthy controls. To this end, we used an adapted version of the DRM paradigm to simultaneously tap memory efficiency, memory errors, and source misattributions.

In keeping with previous work, we expected that relative to controls, schizophrenic patients would display a decrease in memory efficiency and more source misattributions, while showing an increased confidence for these errors. Conversely, control participants were expected to commit more experimentally induced pseudomemories (i.e., false alarms for critical lures) together with an increased confidence for the critical lure words. Most importantly, we tested whether executive dysfunctions related to the prefrontal cortex might statistically predict memory aberrations in schizophrenia.

\section{Method}

\section{Participants}

Twenty-three inpatients (18 men, 5 women) with a DSM-IV (Diagnostic and Statistical Manual of Mental Disorders, $4^{\text {th }}$ ed.; American Psychiatric Association, 1994) diagnosis of schizophrenia were recruited from two Belgian psychiatric hospitals: Medical Centre Sint-Jozef, Munsterbilzen and Public Psychiatric Centre, Rekem. Diagnoses were made by a panel of experienced psychiatrists on the basis of extensive diagnostic interviews, but prior to neurocognitive assessment. Socio-demographic information and clinical data are presented in TABLE 8.1. All patients were on fixed doses of antipsychotic medication, either typical (86\%) or atypical (14\%). Based on a thorough screening of the medical records, they were excluded if they had a history of severe neurological disorders, substance abuse, or another co-morbid axis 1 disorder.

Twenty healthy control participants (18 men, 2 women) were recruited from a pool of volunteers from Maastricht University and through advertisements in a local newspaper. Control participants were matched for age, gender, and level of education. They were screened with a semi-structured interview to rule out a psychiatric history, neurological disorders, alcohol abuse or drug addiction. None of the control participants had a first-degree relative with a history of schizophrenia. Socio-demographic characteristics of the control group are also shown in tABLE 8.1. All participants gave informed consent before participation. They were paid $€ 10$ (approximately 12.5 US dollars) per hour. The study was approved by the standing ethical committee of the Faculty of Psychology, Maastricht University. 
TAble 8.1 Demographic, symptomatological, and neurocognitive characteristics of schizophrenic and control PaRticipants. Standard DEVIATIONS ARE GIVEN IN PARENTHES.

\begin{tabular}{|c|c|c|c|}
\hline & $\begin{array}{l}\text { SCHIZOPHRENIC PATIENTS } \\
\qquad(n=23)\end{array}$ & $\begin{array}{l}\text { CONTROL PARTICIPANTS } \\
(n=20)\end{array}$ & Statistics \\
\hline Age & $36.30(13.13)$ & $35.20(9.71)$ & $t(41)=.31$, n.s. \\
\hline Gender (men/women) & $18 / 5$ & $18 / 2$ & $\chi^{2}(1)=1.08$, n.s. \\
\hline Education level* & $4.74(1.18)$ & $5.35(.99)$ & $t(41)=1.83$, n.s. \\
\hline Intelligence level & $104.45(13.75)$ & $110.75(10.30)$ & $t(41)=1.67$, n.s. \\
\hline Number of hospitalizations & $2.43(1.40)$ & $\begin{array}{ll}-- \\
\end{array}$ & +-- \\
\hline Length of illness (in years) & $6.96(7.38)$ & --- & --- \\
\hline \multicolumn{4}{|l|}{ BPRS } \\
\hline Positive syndrome & $9.17(4.29)$ & --- & --- \\
\hline Negative syndrome & $5.30(2.12)$ & --- & --- \\
\hline Disorganization & $4.13(1.71)$ & --- & --- \\
\hline Total & $32.65(8.52)$ & --- & --- \\
\hline \multicolumn{4}{|l|}{ Executive function } \\
\hline \multicolumn{4}{|l|}{ BADS } \\
\hline Total score ${ }^{* *}$ & $14.04(4.01)$ & $20.05(2.04)$ & $t(41)=5.83, p<.01$ \\
\hline \multicolumn{4}{|l|}{ WCST } \\
\hline $\mathrm{N}$ categories ${ }^{* *}$ & $2.83(1.80)$ & $5.45(1.05)$ & $t(41)=5.72, p<.01$ \\
\hline Perseverative errors \# & $54.67(19.79)$ & $21.10(16.31)$ & $t(41)=5.91, p<.01$ \\
\hline
\end{tabular}

* According to Verhage (1964; Where 1 = LOWER EDUCATION; 7 = UNIVERSITY DEgREe)

* * Lower scores INDicating poorer executive functioning

\# Higher sCores INDicating POORER EXECUTIVE FUNCTIONING

\section{Materials}

Brief Psychiatric Rating Scale (BPRS). We used the 18-item BPRS to measure current symptomatological status of the schizophrenic patients (Overall \& Gorham, 1988). Each BPRS item is rated from 1 (not present) to 7 (extremely present). This scale was originally developed for identifying schizophrenic symptoms, but also includes items tapping, for example, depression. The BPRS was administered by three clinicians who were blind as to the neurocognitive status of the patients. Summing items across subscale yields separate scores for negative, positive, and disorganized thought symptomatology. Thus, a negative symptomatology score is obtained by summing scores for the "emotional withdrawal", "motor retardation", and "blunted affect" items. A positive symptomatology score is obtained by summing across the "unusual thought content", "grandiosity", "suspiciousness", and "hallucinatory behavior" items. The disorganized symptomatology subscale comprises only two symptoms: "conceptual disorganization" and "mannerisms and posturing" (Moritz et al., 2001a).

Premorbid intelligence. Premorbid intelligence was measured with the Dutch Adult Reading Test (Schmand, Lindeboom, \& Harskamp, 1992), which is the Dutch version of the National Adult Reading Test (NART; Nelson, 1982). This task asks participants to read aloud 50 words with irregular spelling. The total score is the number of words that the participant articulates with a correct pronunciation. To estimate premorbid intelligence, scores are compared against normative data. 
Adapted Deese/Roediger-McDermott (DRM) paradigm. We developed an adapted version of the DRM paradigm (Deese, 1959b; Roediger \& McDermott, 1995) so as to tap memory efficiency, memory errors, and source attributions. Participants were administered a Dutch version of the DRM paradigm consisting of 8 selected word lists drawn from a normative study (Chapter 2). Each list consisted of 15 words (e.g., bed, nap, pillow, snooze) semantically related to a non-presented critical lure word (e.g., sleep). The lists were read aloud one after the other. To create an internal-external source attribution condition during encoding of word lists, 4 lists were presented by the experimenter and 4 by the participant (each read aloud). This was done in an alternating sequence, such that the experimenter read the first list, followed by the participant reading the second, then again the experimenter who read the third and so on. For each participant, lists were randomized across this sequence. Words were presented on a Dell 15-inch laptop computer using PowerPoint. Each word was presented for $1 \mathrm{sec}$ with an inter-stimulus interval of $1 \mathrm{sec}$. After the 8 lists had been presented, participants were asked to complete a filler task. Next, participants had an old-new recognition task consisting of the 8 critical lures of the studied lists completely intermixed with 24 study words (the $1^{\text {st }}, 8^{\text {th }}$ and $10^{\text {th }}$ word of each studied list) and 16 unrelated (i.e., new) lures taken from non-presented lists. Only words that were in no way associated with the words in the studied lists were used as unrelated lures. For each of the 48 recognition task items, the participant was instructed to provide three responses: (a) old-new decision; (b) source attribution: That is, when the word was recognized as old, participants had to determine who had read this word aloud: the experimenter or the participant; (c) memory confidence: Here, participants were asked to rate on an 11- point scale (anchors: $0=I$ really don't know; $10=a b$ solutely convinced) how confident they were concerning their old-new and source attribution decisions.

Wisconsin Card Sorting Test (WCST). To tap executive functions, participants were administered a computerized version of the WCST (128 test trials; Heaton et al., 1993). This task was used to measure executive control and working memory (Hartman, Steketee, Silva, Lanning, \& Andersson, 2003). Stimulus material was presented on a Dell 15-inch laptop using E-prime software (www.pstnet.com; Psychology Software Tools). In this task, participants have to sort a deck of cards into four piles, each marked by a key card. Each card consists of one of four designs that appear in one of four different colours (red, green, yellow, or blue), and in one of four forms (triangles, crosses, stars, or circles). Although each card can be sorted according to one of the three dimensions (colour, form or number) at any given point in the test, only one dimension is correct. The sorting rule must be inferred from feedback provided on the computer screen (either "correct" or "incorrect") given after each card. This sorting rule changes without warning after 10 consecutive correct sorts and the test continues until each sorting rule is used twice (in the order colour, form, and number). In 
the present study, we calculated the WCST parameters "categories completed" (0-6), and "number of perseverative errors".

Behavioural Assessment of the Dysexecutive Syndrome (BADS). The BADS (Wilson et al., 1996; see Krabbendam \& Kalff, 1998, for a Dutch version) is a well-established method for assessing executive functions in schizophrenic patients (Krabbendam, de Vugt, Derix, \& Jolles, 1999). It presents the participant with a series of 6 unstructured tasks that are designed to reflect daily situations. These tasks intend to measure planning of behaviour in the face of competing information. The BADS comprises the Rule Shift Cards Test (requiring shifts from simple to complex rules), the Action Program Test (practical problem solving), the Key Search Test (searching strategies), the Temporal Judgment Test (time judgments), the Zoo Map Test (route planning), and the Modified Six elements Test (ability to plan, organize, and monitor behaviour). For each of the tasks, a summary profile score is obtained (Range: $0-4$ ) and these are summed up to obtain a total profile score (maximum $=24$ ), with higher scores indicating better planning capacities. This total profile score will be used in the present experiment.

\section{Procedure}

Participants were tested individually in a quiet room. Before testing, all participants received a semi-structured interview to verify the absence of exclusion criteria. Subsequently, participants were administered the BPRS (patients only), NART, WCST, and BADS. Next, participants were given the following instruction: "In the current experiment, I will present some lists of words to you. Half of the lists will be read by me. For the other half of the lists, I would like you to read aloud the words presented on the computer screen. I will start with the first list, after which you will have to read aloud the next list, followed by a list read aloud by me and so on." This was followed by a filler task (unrelated questionnaire) and finally the recognition test of the DRM was presented.

\section{Statistical analyses}

Three different categories of memory indices were derived: memory efficiency, memory errors, and source attributions. Memory efficiency was defined as the proportion hits. Proportion hits was obtained by dividing the number of recognized old words by the total number of old words on recognition test (i.e., 24). We also calculated proportion false alarms (memory errors), which was defined as number of new words recognized as old divided by 24 . For both categories of new words (i.e., new unrelated words and critical lure words), a false alarm index was also separately calculated. Attributing old words presented by either the experimenter or participant to their correct source was defined as correct source attribution. Accordingly, source misattributions (i.e., incorrect source attributions) were defined as self-presented 
items that were misattributed to the experimenter (self to experimenter) or experimenter-generated items that participants misattributed to themselves (experimenter to self).

Following the Two-High Threshold theory (Corwin, 1994, Snodgrass \& Corwin, 1988), we calculated discrimination index (memory efficiency; $P r$ ) and response bias index (memory errors; $\mathrm{Br}$ ), as measures of accurate and biased discrimination between targets (i.e., old items) and distractors (i.e., new items), respectively. These indices were also calculated for the source attribution measures (source $\mathrm{Pr}$ and $\mathrm{Br}$ ). In doing so, we took the number of erroneous answers into account. Thus, discrimination index was defined as: (number of hits+ .5/number of targets+1)-(number of false alarms+ .5/number of distractors +1 ). Response bias was defined as: (number of false alarms $+.5 /$ number of discractors +1$) /(1$-discrimination index).

For all analyses, significance level was set at $5 \%$; two-tailed. As there was no dependency between recognition variables, two-way Analysis of Variance (ANOVA) with group (control vs. schizophrenia) as between-subject factor and recognition state (hits vs. false alarms critical lures vs. false alarms new) as within-subject factor were carried out. These were followed by independent samples $t$-tests. When dependency was present, independent samples $t$-tests were carried out to compare groups with regard to the different memory indices and confidence ratings. A two-way ANOVA was also carried out for Prindices for the different recognition states, with group status (control vs. schizophrenia) as between-subject factor and discrimination indices (Pr hits-false alarm critical lures vs. Pr hits-false alarms new vs. Pr false alarms critical lures- new) as repeated measure factor. A similar analysis was carried out for the response bias indices ( $B r$ hits-false alarm critical lures vs. $B r$ hits-false alarms new vs. $\mathrm{Br}$ false alarms critical lures-new) Furthermore, for the subsample of schizophrenic patients, correlation analyses (Pearson-product moment) were carried out between BPRS scores and the different memory indices. Multiple linear regression analyses (Enter method) were performed to explore the contribution of neurocognitive functions (BADS, WCST) to memory efficiency (hits and Pr indices), memory errors (false alarms and $\mathrm{Br}$ indices), and source attributions (incorrect source attribution, source $P r$, and source $B r$ ). As measures of effect size, Cohen's $d$ (t-tests) and partial eta squared $\left(\eta_{p}{ }^{2} ;\right.$ Analysis of Variance) were calculated.

\section{Results}

\section{Demographic data}

As can be seen in table 8.1, patients and controls did not differ in terms of gender distribution, age, premorbid intelligence level, and educational level. Test scores of the neurocognitive measures (WCST, and BADS) are also shown.

\section{Encoding conditions}

Before pooling the data across the two encoding conditions (experimenter read vs. 
participant read word lists), the potential confounding effect of encoding conditions on recognition performance of schizophrenic and control participants was tested. A significant interaction between group and encoding condition, with schizophrenic patients being more sensitive to differences in encoding conditions compared to controls would be evidence for such a confounding effect. To test this, two 2 (group status) $\times 2$ (experimenter read vs. participant read word lists) ANOVA's with repeated measures on the last factor were carried out for memory efficiency (hits) and memory errors (total false alarms). The critical interactions remained non-significant [both $F \mathbf{s}$ $(1,41)<2.0$, both $p^{\prime} s>.05$ ], indicating that the two encoding conditions did not produce differential effects on memory efficiency and errors for patients and controls. For this reason, pooling the data across encoding conditions was appropriate.

\section{Memory efficiency and memory errors}

A 2 (group status) $\times 3$ (recognition state: hits vs. false alarms critical lures vs. false alarms new) ANOVA was carried out with repeated measures on the last factor. This yielded a significant main effect of group status $\left[F(1,41)=6.42, p=.01, \eta_{p}{ }^{2}=.14\right]$ and recognition state $\left[F(2,82)=577.49, p<.001, \eta_{p}{ }^{2}=.93\right]$. The interaction effect of group by recognition state was also significant $\left[F(2,82)=10.34, p<.001, \eta_{p}{ }^{2}=.20\right.$; see fIGURE 8.1]. These were followed by independent samples $t$-tests for the memory efficiency and error indices. As can be seen in table $\mathbf{8 . 2}$ (including $t$-values and Cohen's d), schizophrenic patients made significantly fewer hits than controls. These patients also more often made false alarms for new unrelated words as compared to control participants. On the other hand, control participants more often accepted critical lures compared to schizophrenic participants.

\section{Discrimination index (Pr) and response bias index $(\mathrm{Br})$}

Discrimination indices $(P r)$ and response bias indices $(B r)$ were calculated for hits vs. false alarms critical lures, hits vs. false alarms new, and false alarms critical lures vs. false alarms new (based on Hudon, Bellevile, Souchay, Gély-Nargeot, Chertkow, \& Gauthier, 2006). These indices were analyzed using a 2 (group status) $\times 3$ (discrimination state: Prhits-false alarms critical lures vs. $P r$ hits-false alarms new vs. Pr false alarm critical lures-new) ANOVA with repeated measures on the last factor, yielding a significant main effect of group $\left[F(1,41)=24.57, p<.001, \eta_{p}{ }^{2}=.38\right]$ and discrimination state $\left[F(2,82)=327.74, p<.001, \eta_{p}{ }^{2}=89\right]$. However, the interaction effect remained non-significant. This analysis was followed up by independent samples $t$-tests. Groups differed with regard to Prhits vs. false alarms new and Prfalse alarm critical lures vs. new, in that patients were significantly worse at discriminating between these memory indices (see tabl 8.2).

For the response bias indices, a similar 2 (group status) $\times 3$ (response bias state: $B r$ hitsfalse alarms critical lures vs. Brhits-false alarms new vs. Brfalse alarm critical lures-new) ANOVA was analyzed. A significant main effect emerged for the response bias state $[F(2,82)=202.32, p<$ $\left..001, \eta_{p}^{2}=.83\right]$. For the group status, a marginal significant main effect emerged $[F(1,41)=3.71$, $\left.p=.06, \eta_{p}^{2}=.08\right]$. 
TABLE 8.2. MEAN PROPORTION SCORES AND STANDARD DEVIATIONS OF MEMORY EFFICIENCY, MEMORY ERROR, AND SOURCE ATTRIBUTION INDICES FOR SCHIZOPHRENic Patients and healthy control participants. Statistics ( $T$-Values) and efFect sizes (Cohen's $D$ ) are also given.

\begin{tabular}{|c|c|c|c|c|}
\hline & $\begin{array}{c}\text { SCHIZOPHRENIC } \\
\text { PATIENTS } \\
(n=23) \\
M(S D)\end{array}$ & $\begin{array}{l}\text { CONTROL } \\
\text { PARTICIPANTS } \\
(n=20) \\
M(S D)\end{array}$ & $\begin{array}{c}\text { Statistics } \\
\boldsymbol{t}(41)=\end{array}$ & Cohen's $d$ \\
\hline \multicolumn{5}{|l|}{ Memory efficiency } \\
\hline Hits & $.71(.16)$ & $.88(.11)$ & $4.09, p<.001$ & 1.24 \\
\hline \multicolumn{5}{|l|}{ Discrimination index $(\mathrm{Pr})$} \\
\hline Pr hits vs. false alarm critical lures & $-.04(.17)$ & $.03(.13)$ & $1.54, p>.05$ & .46 \\
\hline Pr hits vs. false alarm new & $.59(.17)$ & $.81(.11)$ & $4.96, p<.001$ & 1.54 \\
\hline Pr false alarm critical lures vs. new & $.64(.17)$ & $.78(.16)$ & $2.86, p<.01$ & .85 \\
\hline \multicolumn{5}{|l|}{ Memory errors } \\
\hline False alarm critical lures * & $.78(.16)$ & $.88(.17)$ & $2.02, p=.05$ & .61 \\
\hline False alarm new ** & $.09(.11)$ & $.03(.04)$ & $2.10, p=.04$ & .72 \\
\hline Total false alarm *** & $.32(.10)$ & $.32(.07)$ & $.10, p>.05$ & .11 \\
\hline \multicolumn{5}{|l|}{ Bias index $(\mathrm{Br})$} \\
\hline$B r$ hits vs. false alarm critical lures & $.72(.12)$ & $.86(.12)$ & $3.97, p<.001$ & 1.17 \\
\hline$B r$ hits vs. false alarm new & $.27(.20)$ & $.35(.20)$ & $1.37, p>.05$ & .40 \\
\hline$B r$ false alarm critical lures vs. new & $.30(.21)$ & $.33(.20)$ & $.48, p>.05$ & .15 \\
\hline \multicolumn{5}{|l|}{ Source attribution } \\
\hline Correct source attribution & $.39(.13)$ & $.64(.15)$ & $5.68, p<.001$ & 1.78 \\
\hline Incorrect source attribution & $.32(.12)$ & $.24(.11)$ & $2.17, p=.04$ & .69 \\
\hline Source false alarms Self-Exp. & $.42(.19)$ & $.30(.13)$ & $2.36, p=.02$ & .74 \\
\hline Source false alarms Exp.-Self & $.21(.17)$ & $.18(.15)$ & $.71, p>.05$ & .19 \\
\hline Source $\mathrm{Pr}$ & $.08(.19)$ & $.39(.23)$ & $4.80, p<.001$ & 1.47 \\
\hline Source $\mathrm{Br}$ & $.35(.09)$ & $.41(.07)$ & $2.55, p=.02$ & .74 \\
\hline
\end{tabular}

The interaction between group status and response bias state remained nonsignificant. Overall, both groups showed a liberal response bias $(B r)$ when comparing hits vs. false alarms of critical lures. However, controls displayed a more liberal $\mathrm{Br}$ than schizophrenic patients (see table 8.2). For the other two response biases ( $\mathrm{Br}$ hits vs. false alarms new and $\mathrm{Br}$ false alarms critical lures vs. new), both groups showed a more conservative response bias. However, there were no differences between the two groups on these indices.

\section{Source attribution}

TABLE 8.2 shows source attribution data of both groups, including statistics ( $t$-values) and effect sizes (Cohen's $d$ ). Overall, control participants were significantly better in attributing the correct source to the old items than were patients. Total incorrect source attribution differed between the groups. When differentiating between selfpresented and experimenter-presented words, schizophrenic patients more often attributed self-presented words to the experimenter, compared to control participants. The groups did not differ in attributing words presented by the experimenter to themselves. Groups also differed with regard to Source Pr and source Br. Relative to controls, patients were significantly worse at discriminating between source targets 
and distractors (source $\mathrm{Pr}$ ). However, source response bias (source $\mathrm{Br}$ ) was significantly more liberal for the control group as compared to the schizophrenic group.

\section{Mean proportion scores recognition}

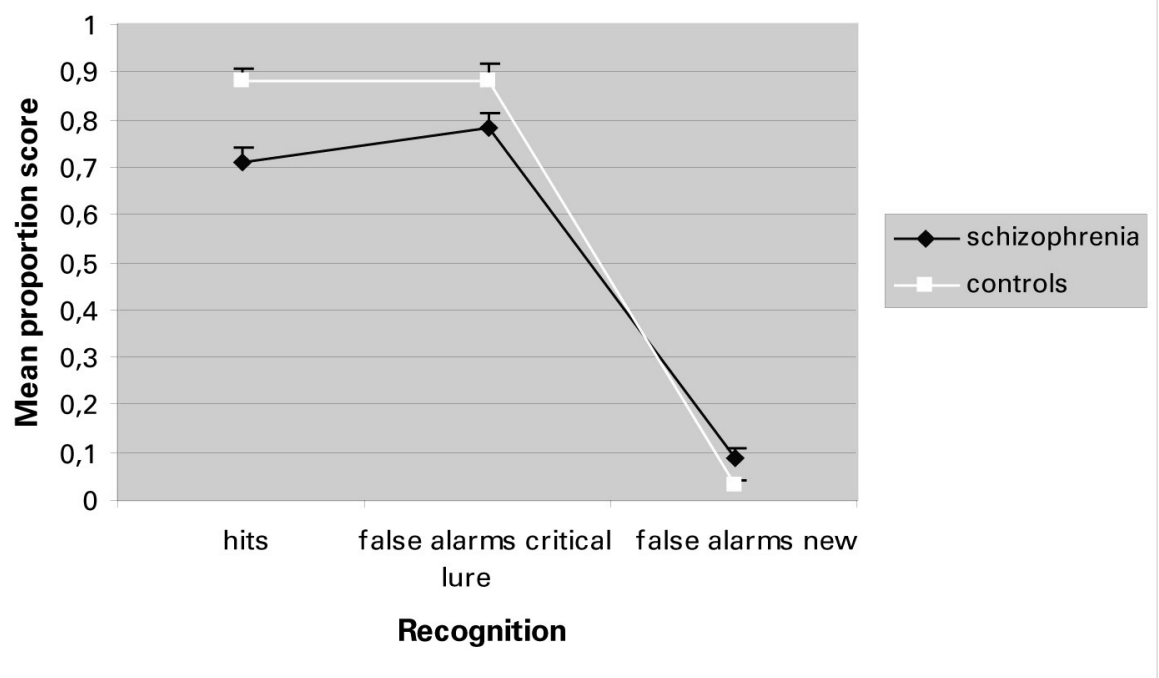

Figure 8.1 MEAN PROPORTION SCORES FOR HITS, FALSE ALARMS OF CRITICAL LURES AND FALSE ALARMS NEW FOR SCHIZOPHRENIC PATIENTS AND CONTROL Participants. Standard errors of MEAN (SEM) ARE PRESENTEd IN T bars.

Relationship between memory efficiency, memory errors, source attribution, and BPRS

None of the symptom scores as measured by the BPRS showed significant correlations with hits. For memory errors, a marginally significant correlation was found between BPRS disorganization and false alarms of new words $(r=.34, p=.05$, onetailed). None of the other memory efficiency, memory errors, or source attribution parameters were related to the BPRS subscales (all $r$ 's $<.20$, all $p$ 's $>.05$ ).

\section{Confidence ratings}

As can be seen in table $\mathbf{8 . 3}$ (including $t$-values and Cohen's $d$ ), relative to patients, control participants had more confidence in their hits. For memory errors, control participants showed significantly more confidence in false alarms for critical lure words compared to schizophrenic patients. As for confidence in source decisions, schizophrenic patients showed higher confidence in falsely attributing old words presented by themselves to the experimenter, whereas controls showed more confidence in correct source attribution. All other comparisons remained non-significant. None of the confidence parameters correlated with BPRS symptom scores. 
TABle 8.3 MeANS (SD) For SOURCE ATtRIBUtion CONFIDENCE IN SCHIZOPHRENIC AND CONTROL PARTICIPANTS.

\begin{tabular}{|c|c|c|c|c|}
\hline & $\begin{array}{c}\text { SCHIZOPHRENIC } \\
\text { PATIENTS }(n=23) \\
M(S D)\end{array}$ & $\begin{array}{l}\text { CONTROLS } \\
\begin{array}{l}(n=20) \\
M(S D)\end{array}\end{array}$ & Statistics & $\begin{array}{c}\text { Cohen's } \\
d\end{array}$ \\
\hline \multicolumn{5}{|l|}{ Confidence memory efficiency } \\
\hline Hits & $6.90(2.18)$ & $8.30(1.25)$ & $2.60, p<.01$ & .79 \\
\hline Correct rejection & $5.27(3.73)$ & $3.83(1.87)$ & 1.56, n.s. & .49 \\
\hline \multicolumn{5}{|l|}{ Confidence memory errors } \\
\hline False alarm critical lure & $5.37(2.03)$ & $7.16(1.82)$ & $2.97, p<.005$ & .92 \\
\hline False alarm new & $2.32(2.72)$ & $1.08(1.61)$ & 1.77 , n.s. & .55 \\
\hline Total false alarm & $4.35(1.84)$ & $5.15(1.35)$ & 1.57 , n.s. & .50 \\
\hline Misses & $6.99(2.27)$ & $5.33(3.21)$ & 1.97 , n.s. & .60 \\
\hline \multicolumn{5}{|l|}{ Confidence source attribution } \\
\hline Correct source attribution & $6.71(2.08)$ & $8.11(1.20)$ & $2.51, p<.02$ & .78 \\
\hline Incorrect source attribution & $6.85(1.91)$ & $6.04(1.86)$ & 1.40, n.s. & .43 \\
\hline Source false alarms Self-Exp. & $7.86(2.69)$ & $5.21(1.17)$ & $4.06, p<.001$ & 1.28 \\
\hline Source false alarms Exp.-Self & $5.84(3.30)$ & $6.29(3.80)$ & .42, n.s. & .13 \\
\hline
\end{tabular}

\section{Neurocognitive deficits and memory functioning}

As can be seen in tABLE 8.1, schizophrenic patients performed worse on the executive function tasks (i.e., WCST and BADS) than controls (all t's $(41)>5.00$, all $p^{\prime} s<.001$ ), all $d$ 's $>1.75$.

We included the neurocognitive measures (i.e., WCST perseverative errors and BADS total score) ${ }^{1}$ into multiple regression analyses (Enter method) to test whether these measures would predict memory efficiency, memory errors, and source attributions (see table 8.4). Data of both groups were pooled and then regression analyses were conducted. The analyses were restricted to memory efficiency (hits, Pr hits-false alarms new, $\operatorname{Pr}$ false alarm critical lures-new), memory errors (false alarms critical lures, false alarms new, and $B r$ hits-false alarms critical lures), and source misattribution (incorrect source attribution, source Self-Experimenter, source Pr, and source $B r)$. The results demonstrated that for hits, WCST perseverative errors and BADS total score were significant predictors. The relationship with perseverative errors was negative, indicating that an increase in perseverative errors was associated with a decrease in memory efficiency. For the BADS test, higher executive functioning scores were associated with an increase in memory efficiency. When these predictors were entered in the regression analysis, the model accounted for $51 \%$ of the variance. The discrimination index ( $P r$ hits vs. false alarms new) was predicted by the BADS total score and WCST perseverative errors. For the BADS score, better executive scores were associated with superior discrimination. For the WCST perseverative errors, heightened perseverative errors were accompanied by poor discrimination. This regression model accounted for $58 \%$ of the variance. None of the executive functioning measures entered the regression equations for the other false alarms and source misattribution scores.

When multiple regression analyses were carried out for both groups separately, significant predictors of memory efficiency only emerged in the schizophrenic 
patient sample. For hits, WCST perseverative errors (negative) and BADS total score (positive) were found to be significant predictors, accounting for $48 \%$ of the variance. Furthermore, BADS total score was a significant predictor of discrimination index ( $P r$ hits vs. false alarms new), with higher BADS scores predicting higher $\mathrm{Pr}$ values, accounting for $47 \%$ of the explained variance. For memory errors and source attribution indices, no significant neurocognitive predictors were identified in both groups.

Table 8.4 Multiple regression analyses predicting memory efficiency (hits, $\boldsymbol{P}_{\boldsymbol{R}}$ hits vs. false alarms new, and $\boldsymbol{P} \boldsymbol{R}$ hits vs. false alarms CITICAL LURE VS. NEW), MEMORY ERRORS (FALSE ALARMS CRITICAL LURES, FALSE ALARMS NEW, BR HITS VS. FALSE ALARMS CRITICAL LURES), AND SOURCE misattribution (incorrect source attribution, source $\boldsymbol{P}_{\boldsymbol{R}}$, and source $\boldsymbol{B}_{R}$ ) scores on the basis of executive dysfunctions (WCST Perseverative ERRORS, BADS tOtal SCORE) For Both GROUPS. ALL INDEPENDENT MEASURES WERE ENTERED.

\begin{tabular}{|c|c|c|c|c|c|}
\hline Dependent VARIABLE & VARIABLES ENTERED AT $p<.05$ & B & SE B & ß & $R^{2}$ \\
\hline \multicolumn{6}{|l|}{ Memory efficiency } \\
\hline \multirow[t]{2}{*}{ Hits } & Perseverative errors & -.05 & .02 & -.36 & .51 \\
\hline & BADS total score & .35 & .14 & .42 & \\
\hline \multirow[t]{2}{*}{ Pr hits vs. false alarms new } & Perseverative errors & -.002 & .001 & -.35 & .58 \\
\hline & BADS total score & .02 & .01 & .47 & \\
\hline Pr false alarms critica lures vs. new & No variables entered & & & & \\
\hline \multicolumn{6}{|l|}{ Memory errors } \\
\hline False alarm critical lures & No variables entered & & & & \\
\hline False alarm new & No variables entered & & & & \\
\hline \multirow[t]{2}{*}{$B r$ hits vs. false alarms critical lures } & No variables entered & & & & \\
\hline & BADS total score & & & & \\
\hline \multicolumn{6}{|l|}{ Source attribution } \\
\hline Incorrect source attribution & No variables entered & & & & \\
\hline Source Self-Experimenter & No variables entered & & & & \\
\hline Source $\operatorname{Pr}$ & No variables entered & & & & \\
\hline Source $B r$ & No variables entered & & & & \\
\hline
\end{tabular}

\section{Discussion}

We found that patients with schizophrenia, relative to controls, showed poorer memory efficiency. Their hit rates were accompanied by a lowered confidence in comparison to the control group. As to false alarms of critical lures, in line with prior research (e.g., Roediger \& McDermott, 1995; Elvevåg et al., 2004; Moritz et al., 2004), both groups showed a substantial number of such false alarms, and also demonstrated at chance performance for discrimination levels between hits and false alarms of critical lures. However, and again replicating previous work (Moritz et al., 2004; Weiss et al., 2002), it was the control group that had the highest level false alarms of critical lures. This was accompanied by heightened confidence. On the other hand, schizophrenic patients more often made false alarms for new lures than controls, therefore also showing decreased discrimination between hits and false alarms for new words. As to the individual source misattribution indices, our findings concur with previous 
research by Keefe et al. (1999), Moritz et al. (2003), and Vinogradov et al. (1997) in that relative to healthy controls, schizophrenic patients were more prone to recognize self-presented words as presented by the experimenter (internal-external source misattribution) with increased confidence ratings. This misattribution tendency was clearly reflected in the source discrimination index, which was significantly lower in the schizophrenic patients than controls (see also Brébion et al., 2002; Henquet, Krabbendam, Dautzenberg, Jolles, \& Merckelbach, 2005). Another important finding of the current study is that executive functioning indices (i.e., WCST perseverative errors and BADS total score) serve as significant predictors of memory efficiency. Yet, none of these neurocognitive measures accounted for source misattributions or memory error scores. As well, in schizophrenic patients, but not in controls, executive dysfunctions predicted memory efficiency.

When overall performance accuracy was taken into account, controls showed a more liberal response bias tendency than schizophrenic patients for hits vs. false alarms of critical lures. For source $B r$, the magnitude of this bias index is similar to those reported in previous research using similar samples (e.g., Brébion et al., 2002; Henquet et al., 2005). However, previous studies did not detect significant differences in response bias between schizophrenic patients and healthy controls. The fact that we did find such differences is probably related to the DRM version that we employed. Thus, the high levels of false alarms of critical lures in particularly controls is most likely the result of a more liberal response bias in this group promoted by the semantically related stimulus material. In contrast, schizophrenic patients' higher false alarm levels for new lures might reflect overall inaccuracy of source monitoring performance, as a more conservative response bias was found for these measures.

As to pseudo-memories, schizophrenic patients did not falsely recognize critical lures more often than healthy controls. As a matter of fact, the opposite was true. Several studies have described spreading activation in semantic networks as a key feature in eliciting experimentally induced pseudo-memories (e.g., Roediger, Balota, $\&$ Watson, 2001). When presented a list of semantically associated words, semantically related, but non-presented critical lure words will also be activated. It may well be the case that this spreading activation is less marked in schizophrenic patients, resulting in only weak activation of the semantic associate and, as a consequence, reduced levels of false recognition of critical lures (see also Moritz et al., 2004; Elvevåg et al., 2004). A closely related view is that the lower levels of pseudo-memories in schizophrenic patients have to do with their difficulty in dealing with context and redundant information (e.g., Waters, Maybery, Badcock, \& Michie, 2004). Thus, control participants would profit from the context of semantically related material, but the other side of the coin is that this makes them vulnerable to this specific class of pseudo-memories. To the extent that schizophrenic patients can not profit from semantic context, this makes them relatively immune to this category of pseudo-memories. Still another possibility may be that encoding deficits not only lead to a decrease 
in correct recognition of old words, but may also reduce semantically related false recognition, because lack of stable memory representations means that the weak memory traces can not converge onto the critical lure word. ${ }^{2}$

As for source misattributions, we found that relative to controls, schizophrenic patients more often identified self-presented words as presented by the experimenter. This externalizing tendency has also been found in earlier work (e.g., Moritz et al., 2003). Replicating Moritz et al. (2003), this source misattribution was not correlated with positive symptoms or poor executive functioning. It is true that control participants also committed source misattributions. However, their lowered confidence ratings indicate that they attached some sort of "non-trustworthiness" tag to their misattributions. Another antecedent of controls' misattributions might be their source response bias. In contrast, schizophrenic patients make more source misattributions and they do this with high confidence. One important antecedent in making correct source attributions is the amount of perceptual detail/contextual information that is encoded, with correct memory traces exhibiting many distinctive features (e.g., Johnson et al., 1993). Schizophrenic patients show clear deficits in the encoding of distinctive perceptual features (Brébion et al., 2005). Meanwhile such features serve as landmarks in classifying an event as presented by themselves or by external sources. In schizophrenic patients, these landmarks are lacking, making them more vulnerable to this specific type of source misattribution.

Previous research by Brébion et al. (2005) found poor correct recognition in schizophrenia to be related to slower processing speed and impaired selective attention. These impairments in automated cognitive functions may undermine efficient encoding, thereby impeding serial learning and semantic organization of, for example, word lists. The present study shows that executive control, as an effortful process, also plays a role in recognition memory. Although this factor significantly predicted memory efficiency when both subsamples were pooled, its predictive value was mainly carried by the group of schizophrenic patients. Executive dysfunctions (increase in perseverative errors during WCST and poor planning function during BADS) in schizophrenic patients have been well documented and there is every reason to suspect that they originate from reduced activity in the prefrontal cortex (e.g., Li, 2004; Seidman et al., 1994). Interestingly, damage to the prefrontal regions - reflected in executive dysfunctions and working memory impairments - results in poor recollection of the contextual details (distinctive perceptual features) of previously learned information (e.g., Chapter 1). Indeed, the most parsimonious explanation for the pattern found in the current study is that schizophrenic patients' problems with executive control and planning leads to poor binding and difficulties in holding online the set of semantic associates in the word lists. Eventually, this results in poor encoding (superficial processing) of the word lists. Indeed, executive control and working memory are important in organizing and clustering networks of semantic associates. Problems with executive control and working memory will subsequently 
lead to lower memory efficiency. Thus, our findings show that an extension of the current model by Brébion and co-workers (2005) is required in that not only automated cognitive functions (i.e., slower processing speed and selective attention) affect memory efficiency, but also effortful neurocognitive functions (i.e., executive dysfunctions).

In the current study, we found that poor executive functioning, as indexed by WCST and BADS, was linked to poor memory efficiency. It might well be the case that this link is highly specific. That is to say, performance on the WCST and BADS critically depends on semantic processing (e.g., Heaton et al., 1993), and thus one could argue that poor semantic processing, rather than poor executive functioning per se, accounts for the link between performance on our executive functioning tasks and memory efficiency. Clearly, this point warrants further study. More precisely, future research could profit from including semantic processing measures (e.g., semantic fluency tests) along with executive functioning tasks to further delineate the specific contributions of poor semantic processing abilities and executive deficits to schizophrenic patients' memory aberrations.

In contrast to previous research (Brébion et al., 2000, 2002), we did not find a significant relationship between positive symptomatology and specific (source) memory errors. This could be due to the fact that schizophrenic patients in this study were mostly stabilized chronic patients. As a group, patients exhibited little variability in BPRS scores. Future research on how positive symptoms relate to source misattributions, with larger and more diverse samples, seems to be necessary.

One limitation of the present study was that all our schizophrenic patients were receiving psychotropic medication. Theoretically, differences in memory performance between patients and healthy controls could be confounded by medication. However, research on the impact of medication status on episodic memory has produced mixed results, with most recent studies reporting no significant effect (e.g., Aleman et al., 1999; Mortimer, 1997) or even cognitive benefits (Hagger, Buckley, Kenny, Friedman, Ubogy, \& Meltzer, 1993).

A second limitation is that we compared memory performance of schizophrenic patients to that of healthy controls. There are other clinical groups that are known to exhibit memory dysfunctions (e.g., memory distrust in obsessive-compulsive disordered patients; Radomsky, Gilchrist, \& Dussault, 2006). Thus, further research in this domain should preferably include other clinical groups, although the practical difficulties of this are obvious.

Finally, one could argue that in combination with the small sample, our procedure of 8 word lists subdivided into two encoding conditions, might not have been sensitive enough (i.e., was underpowered) to detect group differences in all types of false alarms. However, when comparing our findings with previous studies looking at memory functioning in schizophrenia (e.g., Elvevåg et al., 2004; Keefe et al., 1999; Moritz et al., 2003, 2004; Vinogradov et al., 1997; Weiss et al., 2002), the similarities 
in the pattern of memory aberrations (i.e., lowered efficiency, fewer false alarms of critical lures, internal-external source misattributions) documented for schizophrenic patients is striking. Nonetheless, it would be wise to increase sample size and the number of word lists and related lure words in future research.

To our knowledge, this is the first study that made an attempt to document the full range of memory aberrations of schizophrenic patients with one paradigm. Recognition memory (efficiency, errors, and source attribution) was found to be markedly impaired in patients with schizophrenia, with executive dysfunctions better predicting memory efficiency than symptomatology. These findings provide further evidence for explaining schizophrenics' memory impairments in terms of fundamental neurocognitive deficits like executive dysfunctions. 



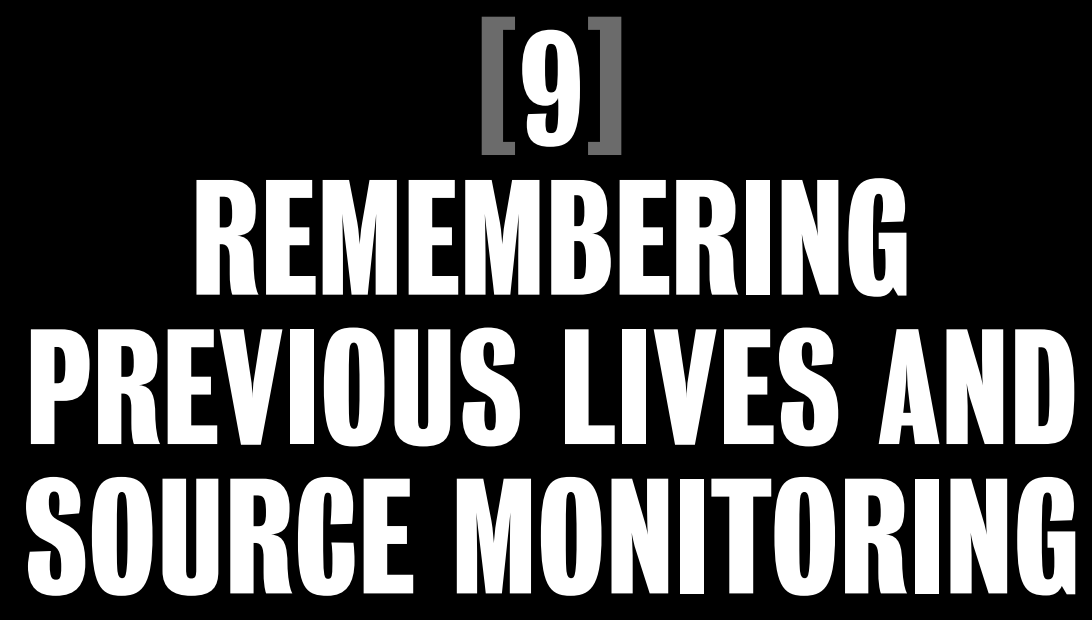





\title{
CHAPTER [9] REMEMBERING PREVIOUS LIVES AND SOURCE MONITORING
}

An adjusted version of this chapter is published as: Peters, M.J.V., Horselenberg, R., Jelicic, M., \& Merckelbach, H. (2007). The false fame illusion in people with memories about a previous life. Consciousness and Cognition, 16, 162-169.

\begin{abstract}
The present study examined whether individuals with full-blown memories of highly implausible events are prone to commit source monitoring errors. Participants reporting previous-life memories and those without such memories completed a false fame task. This task provides an index of source monitoring errors (i.e., misclassifying familiar non-famous names as famous names). Participants with previous-life memories had a greater tendency to judge the names of previously presented nonfamous people as famous than control participants. The two groups did not differ in terms of correct recognition of new non-famous names and famous names. Although dissociation, cognitive failures, sleep-related experiences, depressive symptoms, and signs of psychological distress were all significantly higher in participants with previous-life memories than in controls, these variables did not predict the false fame illusion.
\end{abstract}




\section{Introduction}

A plethora of research has demonstrated that adults can create autobiographical memories for events that never occurred (e.g., Clancy, McNally, Schacter, Lenzenweger, \& Pitman, 2002; Loftus \& Pickrell, 1995; Mazzoni, Loftus, \& Kirsch, 2001). According to Hyman and Kleinknecht (1999), the development of such pseudo-memories is dependent on different cognitive processes, which can interact with each other. For example, whether pseudo-memories occur is partly dependent on the plausibility of the suggested event (e.g., Pezdek, Finger, \& Hodge, 1997; Scoboria, Mazzoni, Kirsch, \& Relyea, 2004; Smeets, Merckelbach, Horselenberg, \& Jelicic, 2005). Once an event is perceived as plausible, individuals may start to believe that the event has happened to them. Another process in the road to a pseudo-memory is repetition of an assertion/ plausible event, which can increase the confidence in the truth of this assertion/ event. This is known as the "illusion of truth." (Bacon, 1979; Hasher, Goldstein, \& Toppino, 1977; Hertwig, Gigerenzer, \& Hoffrage, 1997). A next possible step is that individuals interpret their thoughts and fantasies about the fictitious event as real memories (Mazzoni et al., 2001; see also Scoboria et al., 2004; Smeets et al., 2005). In the pathway to a pseudo-memory, source monitoring errors (i.e., failure to attribute the correct source to information) are a decisive factor and can creep in anywhere along the way (e.g., Johnson, Hashtroudi \& Lindsay, 1993).

Using well-researched laboratory paradigms to elicit false recall of e.g., words (the Deese/Roediger-McDermott task; see below), several studies have shown that women reporting recovered memories of childhood sexual abuse are more prone to memory distortions than control participants (e.g., Clancy, Schacter, McNally, \& Pitman, 2000; Geraerts, Smeets, Jelicic, van Heerden, \& Merckelbach, 2005). However, in studying these populations, it is very difficult to establish the ground truth, i.e., to determine whether the recovered memories in these samples are genuine or false. Thus, one can never be certain that their susceptibility to memory distortions is a function of cognitive impairments related to a history of abuse or the manifestation of a trait-like tendency to develop pseudo-memories (see also Clancy et al., 2002). For this reason, researchers have tried to find specific groups of people who report memories of events that seem very implausible to have happened. A case in point is a series of studies by Clancy and co-workers (2002; see also McNally \& Clancy, 2005; McNally, Lasko, Clancy, Macklin, Pitman, \& Orr, 2004), who examined whether people claiming to be abducted by aliens are more likely to commit source monitoring errors than people without such memories. Participants with alien abduction experiences and control participants were given a variant of the Deese/RoedigerMcDermott paradigm (DRM paradigm; Deese, 1959b; Roediger \& McDermott, 1995) to examine their propensity to falsely recall and recognize critical lure words that had never been presented. Briefly, in the DRM paradigm participants are given lists of semantically related words (e.g., bed, pajama) that refer to non-presented critical lure words (e.g., sleep). In subsequent memory tests, some participants claim to re- 
member the non-presented critical lure words. The most likely explanation for this is that they mistake internally generated associations for memories of real words. Thus, the DRM task taps source monitoring errors. Clancy and colleagues (2002) found that participants reporting memories of alien abduction or the belief that they had been abducted exhibit a greater tendency to falsely recall and recognize non-presented critical lure words in the DRM paradigm than control participants. Specific cognitive characteristics like hypnotic suggestibility, depressive symptoms, and schizotypal features were found to be significant predictors of false recall and false recognition. Thus, there is reason to believe that people with this particular type of implausible autobiographical memories have source monitoring problems.

Another class of autobiographical memories that are highly implausible are hypnotically induced memories about previous lives. Although spontaneous previous-life memories are in some countries part of a broad culture (e.g., Sri Lanka; Haraldsson, 2003), in modern Western societies such memories are often elicited under hypnosis (e.g., Spanos, Menary, Gabora, DuBreuil, \& Dewhirst, 1991).

The current study aimed at a conceptual replication of Clancy et al.'s (2002) findings. Focussing on a different type of implausible memory and relying on a different type of source monitoring task (see below), we tried to replicate the basic findings of Clancy et al. (2002) that reports of implausible memories are associated with a tendency to commit source monitoring errors. To this end, we examined source monitoring errors in people reporting hypnotically induced previous-life memories and control participants. The paradigm we employed was the false fame paradigm (Jacoby, Kelley, Brown, \& Jasechko, 1989). In this paradigm, participants are first asked to read out aloud a series of non-famous names. The next day, participants receive a list of names, consisting of the old non-famous names, new non-famous names, and also names referring to famous people (e.g., actors, writers, politicians). Participants are instructed to make fame judgments for each presented name. A standard finding in this paradigm is that participants falsely identify previous studied (old) non-famous names as famous names (Jacoby et al., 1989). This illusion stems from participants' tendency to mistake the familiarity of an old non-famous name as an indication that the person must be famous.

A subsidiary aim of our study was to explore to what extent certain personality traits predict the false fame illusion. Previous research has shown that traits like absorption (Clancy et al., 2002), fantasy proneness (e.g., Spanos et al., 1991), dissociation (Eisen \& Lynn, 2001), and sleep disruptions (McNally \& Clancy, 2005) are more pronounced in people who are susceptible to pseudo-memories. To explore the relationship between these traits and source monitoring errors in participants with previous-life memories, we administered measures of dissociation, fantasy proneness, cognitive failures, sleep experiences, and general psychopathology to our sample. 


\section{Method}

\section{Participants}

Previous-life participants were recruited through contacts with 6 reincarnation therapists in the Maastricht area, which is the most southern part of the Netherlands. Thirteen clients ( 2 men) claiming previous-life memories volunteered to participate in our study. Mean age of these participants was 44.7 years ( $S D=9.70$; Range: 29), with mean years of education being 14 years $(S D=1.40)$. The control group, which was recruited through advertisement in local newspapers, consisted of 11 women and 2 men, matched as much as possible on age (mean age $=44.9, S D=9.20$; Range: 30 ) and years of education $(M=14.2, S D=1.40)$; both $t$ 's $(24)<1.0$. Before participants completed the questionnaires and the false fame task (see below), both groups were given a set of questions concerning their beliefs in previous lives and reincarnation therapy. In the previous-life group, reincarnation therapy comprised of hypnosis-like sessions, in which the patients were asked to lay down and concentrate on their inner feelings. On average, previous-life participants had had 20.1 regression therapy sessions (Range: 4-95 sessions). Sixty-nine percent of those previous-life participants had clear, detailed, and vivid memories of their previous lives, with all of the previous-life participants believing that previous lives do exist. None of these participants had, prior to therapy, any memory about their previous lives. The previous-life participants recovered at least 5 distinct previous lives. Sixty-one percent of the previouslife accounts were highly aversive and pertained to negative events (e.g., murder, torture, war). In contrast to the previous-life participants, none of the control participants believed in the existence of previous-life experiences or had been subjected to reincarnation therapy.

\section{Materials and procedure}

The experiment was approved by the standing ethical committee of the Faculty of Psychology, Maastricht University. Participants were tested individually in a quiet laboratory room. Upon arrival, participants were asked to sign an informed consent form. Instructions, manipulations, and stimulus materials were given on paper, except for the false fame paradigm, which was presented via a computer. Participants completed the Dissociative Experiences Scale (DES; Cronbach's $\alpha=.90$; Bernstein \& Putnam, 1986), Creative Experiences Questionnaire (CEO; Cronbach's $\alpha=.81$; Merckelbach, Horselenberg, \& Muris, 2001), Cognitive Failures Questionnaire (CFO; Cronbach's $\alpha=.90$; Broadbent, Cooper, Fitzgerald, \& Parkes, 1982), lowa Sleep Experiences Scale (ISES; Cronbach's $\alpha=.85$; Watson, 2001), and the Symptom Check List-90 (SCL-90; Cronbach's $\alpha=.87$; Derogatis, Richels \& Rock, 1976). Participants also underwent an adapted version of the false fame task (Jacoby et al., 1989). To control for order and fatigue effects, questionnaires and false fame task were counterbalanced across participants. 
Dissociative Experiences Scale (DES). The DES is a 28 item self-report measuring dissociative phenomena like feelings of depersonalization, derealization, and psychogenic amnesia. Participants are asked to indicate on $100 \mathrm{~mm}$ visual-analog scales (VAS; anchors: $0=$ not at all; $100=$ very much) to what extent they experience these phenomena on a daily basis. A total DES score is derived by averaging the scores across individual items (Range: 0 - 100), with higher total DES scores indicating stronger dissociative tendencies.

Creative Experiences Questionnaire (CEQ). The CEQ consists of 25 true/false items. CEO items were derived from the extensive case descriptions of fantasy proneness provided by Wilson and Barber (1982). An illustrative item is "My fantasies are so vivid that they are like a good movie". A total score (Range: $0-25$ ) is obtained by summing the true-answers, with higher scores indicating higher levels of fantasy proneness.

Cognitive Failures Questionnaire (CFQ). The CFQ consists of 25 items measuring the frequency of everyday failures of memory, attention, action, and perception. Participants have to indicate on a 5-point scale how often they experience particular cognitive lapses and blunders (e.g., forgetting appointments; anchors: $0=$ never, $4=$ very often). Scores are summed to obtain a total CFO score, with higher scores indicating a higher frequency of self-reported cognitive failures.

lowa Sleep Experiences Scale (ISES). The ISES is an 18-item questionnaire that asks participants to rate the frequency of various sleep- and dream-related experiences (e.g., nightmares, etc.) on a 7-point scale (anchors: $1=$ never, $7=$ several times a week). A mean score can be obtained by summing across all items.

Symptom Check List-90 (SCL-90). A Dutch version of the SCL-90 was used. This scale comprises 90 items and is a self-report measure of current psychological symptoms. Each item taps one of 9 clinical domains (e.g., anxiety, depression, somatization, insufficiency, etc.). Items describe symptoms and participants rate on a 5-point scale to what extent they have experienced the symptoms in the last week (anchors: $1=$ not at all; $5=$ extremely). A total SCL-90 score can be derived by summing across items. This total SCL-90 score is an index of general psychopathology.

False FameTask. An adapted version of the Jacoby et al. (1989) false fame task was used. In extensive studies in our lab, we documented that this version elicits the false fame illusion in a non-trivial minority of healthy participants (Horselenberg, Merckelbach, Wessel, Verhoeven, \& Zeles, 2006). In our version of the task, participants are given the second part of the false fame paradigm $2 \mathrm{hrs}$ after the study part (see below) rather than $24 \mathrm{hrs}$ after the study part as was the case in the original experiment (Jacoby et al., 1989). 
Participants were informed that they would participate in a pronunciation test. Next, they went through the study phase of the task, during which they were asked to read a series of 40 non-famous names (hereafter referred to as old non-famous names), speaking aloud in a microphone placed in front of them. During this phase, no responses were recorded. Selection of names was based on extensive pilot work. In total, a set of 160 names was selected. Half of them referred to non-famous persons, while the other half referred to moderately famous Dutch persons. Both name types were matched on length of first and family name and on gender. Names were presented on a Dell computer running Experimental Run Time System (ERTS; Beringer, 1996) on a Philips Brilliance $17^{\prime}$ screen. Names were presented for $2 \mathrm{sec}$ in lowercase letters with the initial letters of the first and family name in capitals. This was followed by a $2 \mathrm{sec}$ interstimulus interval. The second phase was a test phase (famous/non-famous discrimination task) and took place $2 \mathrm{hrs}$ after the study phase. Participants were explicitly instructed that old non-famous names would be presented along with new non-famous names and famous names. Participants were told that they did not have to provide a reason for their subjective fame-judgments. After 16 practice trials, they were exposed to a series of 160 names: 40 old non-famous names, 40 new non-famous names, and 80 famous names. By pressing either the right or left shift key of the keyboard, participants indicated for each name whether it was a famous or non-famous name. Names (first name and family name) were selected quasi-randomly from the pool of 160 names. Presentation of each name was contingent upon participants pushing one of the shift keys. The name disappeared from the screen as soon as the participant responded. This was followed by a $1 \mathrm{sec}$ blank screen before the next name appeared. The computer recorded each fame judgment. Within the previous-life and control group, shift keys (i.e., left vs. right) and sequence of the three types of names were counterbalanced. We calculated proportions of old and new non-famous names that participants misclassified as famous (i.e., dividing the number of non-famous names judged as famous by 40 ).

\section{Results}

Mean proportions of old and new non-famous names misclassified as famous in each group is shown in figure 9.1. A 2 (group: previous-life vs. control) x 2 (false fame: old vs. new non-famous names) Analysis of Variance (ANOVA) with repeated measures on the last factor yielded a significant false fame effect $[F(1,24)=9.70, p=$ $.005, \mathrm{MSE}=17.16, \eta^{2}=.29$ ] and a borderline significant main effect of group; $F(1$, $24)=3.18, p=.08, \mathrm{MSE}=23.99, \eta^{2}=.19$. Most importantly, the critical group $x$ false fame interaction was significant $\left[F(1,24)=5.03, p=.03\right.$, MSE $\left.=17.16, \eta^{2}=.17\right]$, due to the fact that compared to controls, previous-life participants misinterpreted more old non-famous names as famous. Thus, our data show that the previous-life group displayed a stronger false fame illusion than the control group. Another way to approach this issue is by calculating a false fame index. This index can be obtained by 
subtracting the proportion of new non-famous names judged as famous from the proportion old non-famous names judged as famous (Range: -.58 to .10; see Rybash, Rubenstein, \& DeLuca, 1997). Again, the difference between the previous-life group and control group was significant; $t(24)=2.3, p=.034$; Cohen's $d=.82$. Participants with previous-life experiences did not differ significantly from controls with regard to the proportion hits (famous names judged as famous), means being .86 ( $S D=.09$ ) and $.87(S D=.11)$, respectively; $t(24)<1$.

TABLE 9.1 shows mean scores on the self-report questionnaires. The previous-life group scored significantly higher on the DES $[t(24)=2.70, p<.01$; two-tailed], CFO $[t(24)=2.40, p=.02$; two-tailed $]$, ISES $[t(24)=3.15, p<.01$; two-tailed $]$, and SCL-90 $[t(24)=3.50, p=.002$; two-tailed $]$. Furthermore, previous-life participants scored significantly higher on all SCL-90 subscales (e.g., depression, anxiety, etc.; see table 9.1) [all t's (24) > 2, $p<.05$; two-tailed], except for hostility and sleeplessness; both t's (24) $<1.0$, both $p$ 's $>.05$. As for the CEQ, previous-life participants had marginally higher scores than control participants; $t(24)=1.70, p=.09$.

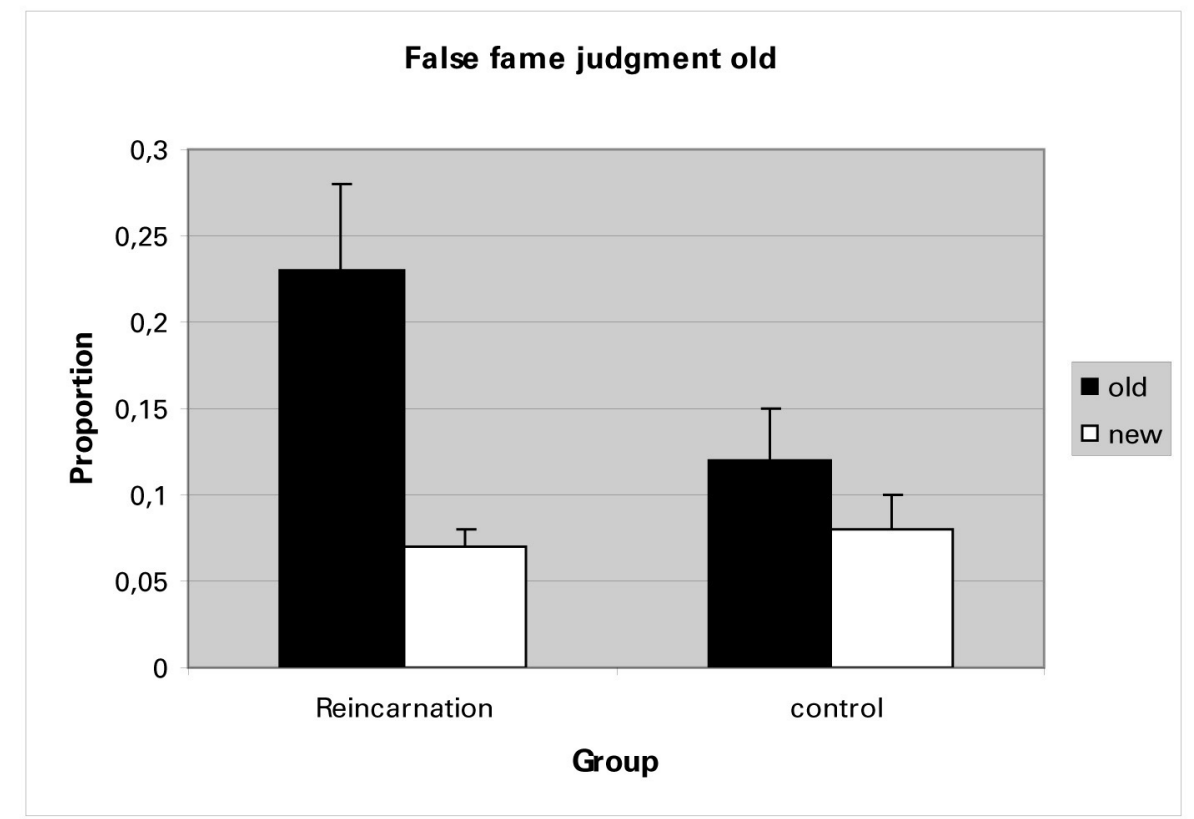

Figure 9.1 Proportion of old AND NeW NON-FAMOUS NAMES Misclassified AS FAMOUS FOR the PREVIOUS-LIFE MEMORIES GROUP AND the CONTROL GROUP (SEM'S ARE GIVEN IN T-BARS; $N=\mathbf{2 6}$ ). 
To explore the relationship between personality traits and the false fame index, Pearson product-moment correlations were calculated for data collapsed across groups and for both groups separately. As can be seen in tABLE 9.2, when the two groups were collapsed, only the lowa Sleep Experiences Scale was marginally associated with the false fame index. None of the trait measures were significantly related to the hit rate (famous names as famous); all $r$ 's < .40, all $p$ 's > .05; two-tailed. When looking at each group separately, Pearson product-moment correlations between the false fame index and the various personality traits remained non-significant; all $r$ 's $<.40$, all $p$ 's > .05; two-tailed. ${ }^{1}$ Similarly, non-significant correlations were found between hit rate and personality traits.

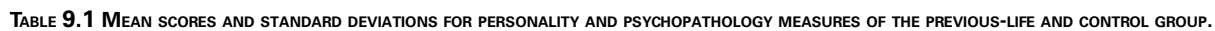

\begin{tabular}{|c|c|c|c|c|}
\hline & \multirow{2}{*}{$\begin{array}{c}\text { PREVOUS-LIFE } \\
\begin{array}{c}(n=13) \\
M\end{array}\end{array}$} & \multicolumn{3}{|c|}{$\begin{array}{l}\text { CONTROLS } \\
(n=13)\end{array}$} \\
\hline & & $S D$ & $M$ & $S D$ \\
\hline Dissociative Experiences Scale & 18.90 & 11.70 & 9.70 & 4.90 \\
\hline Creative Experiences Questionnaire & 9.20 & 4.20 & 6.30 & 4.40 \\
\hline Cognitive Failures Questionnaire & 41.60 & 12.60 & 30.50 & 10.60 \\
\hline lowa Sleep Experiences Scale & 50.38 & 11.81 & 36.00 & 11.51 \\
\hline Total score SCL-90 & 141.90 & 37.80 & 101.90 & 19.60 \\
\hline SCL-90 Agoraphobia & 9.08 & 2.50 & 7.46 & 0.97 \\
\hline SCL-90 Anxiety & 17.69 & 6.91 & 11.77 & 2.52 \\
\hline SCL-90 Depression & 28.23 & 7.39 & 20.23 & 4.85 \\
\hline SCL-90 Somatization & 20.08 & 6.09 & 14.69 & 2.98 \\
\hline SCL-90 Insufficiency & 17.38 & 5.61 & 12.85 & 4.14 \\
\hline SCL-90 Sensitivity & 33.54 & 12.80 & 22.92 & 4.97 \\
\hline SCL-90 Hostility & 8.23 & 2.09 & 6.92 & 1.50 \\
\hline SCL-90 Sleeplessness & 7.61 & 4.07 & 5.31 & 2.78 \\
\hline
\end{tabular}

Note: DES = Dissociative Experiences Scale (Bernstein \& Putnam, 1986; range: 43); CeQ = Creative Experiences Questionnaire (Merckelbach et al., 2001; range: 16); CFQ = Cognitive Failures Questionnaire (Broadbent et al., 1982; range: 50); ISES = lowa Sleep Experiences Scale (Watson, 2001; range: 44); SCl-90 = Symptom Check List-90 (Derogatis et AL., 1976; RANGE: 177)

TAble 9.2 Correlations between Personality and psychopathology measures, false fame indeX and hit rates on famous names, COLLAPSED ACROSS GROUPS.

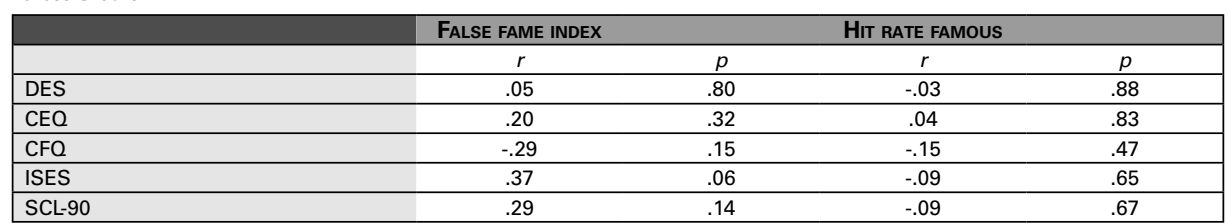

Note: DES = Dissociative Experiences Scale; CEQ = Creative Experiences Questionnaire; CFQ = Cognitive Failures Questionnaire; ISES = lowa Sleep Experiences Scale; SCL-90 = Symptom Check List-90.

\section{Discussion}

The present study examined source monitoring in participants with highly implausible autobiographical memories and control participants. Our results show that participants with previous-life memories had a stronger tendency to judge names of previously presented non-famous people as famous than controls. In line with Clancy et al. (2002), we found that memories of highly unlikely events are associated with elevated levels of depression (as measured by the SCL-90 depression subscale) 
and other signs of psychological distress. That is, participants with memories of previous lives reported heightened levels of dissociation, sleep disturbances, cognitive failures, and, to a lesser extent fantasy proneness. However, in contrast to Clancy et al. (2002), we found only sleep disturbances (ISES) to be related to the false fame index. In future research, it would be informative to explore whether the specific content of these previous-life memories (amount of detail, vividness of detail, etc.) or the confidence in these memories might be possible moderators in the relationship between the false fame effect and the various personality and psychopathology characteristics. Such moderator variables might explain the discrepancies between our data and those of Clancy et al. (2002). Since no detailed description of these factors was given by the previous-life participants, we can only speculate about how, for example, eccentricity of previous-life memories might modulate the link between previous-life memories and dissociation or fantasy proneness.

Our finding that people with previous-life memories exhibit a relatively robust false-fame illusion is important because this may help to explain how these people come to accept their previous-life memories as genuine memories. When individuals with a tendency to make source monitoring errors are repeatedly asked to imagine events that supposedly took place during past lives, even highly implausible events suggested by the therapist may come to generate feelings of familiarity (as indicated in this experiment by the false fame effect; see Jacoby et al., 1989). This line of reasoning fits nicely with what is known as the "illusion of truth", whereby reiterating assertions make these assertions more believable (Arkes, Hackett, \& Boehm, 1989; Bacon, 1979; Begg, Anas, \& Farinacci, 1992; Hertwig et al., 1997). Research by Hasher et al. (1977) and Bacon (1979) demonstrated that repetition of an assertion and/or the recognition of an assertion as old, increases the confidence in its truth. This effect is independent of the actual truth of the assertion. In a related vein, familiarity (which automatically increases with repetition; Begg et al., 1992) may erroneously be interpreted as a diagnostic sign of the truth status of the event (see also Chapter 1). Indeed, Hyman and Kleinknecht (1999) argue that another possible cognitive process in the development of pseudo-memories is plausibility, which is of course closely related to familiarity. Even if it is highly unlikely, once an event is perceived as plausible, individuals can acquire the autobiographical belief that the event has happened to them. Another possible step would be the acceptance of their thoughts and fantasies about the event as genuine memories (Mazzoni et al., 2001; see also Scoborria et al., 2004; Smeets et al., 2005). One decisive factor in these cognitive processes is source monitoring (Johnson, Hashtroudi \& Lindsay, 1993), because source confusion can lead to acceptance of internally generated events as externally perceived.

It is likely that pseudo-memories in the clinical context involve source monitoring errors (e.g., Hyman \& Kleinknecht, 1999). It is also evident that the false fame effect is a pure laboratory manifestation of a source monitoring error (Multhaup, De Leonardis \& Johnson, 1999). Things are quite different with most autobiographi- 
cal retrieval tasks (e.g., diary studies). Nevertheless, some authors have argued that small scale laboratory tasks tapping memory illusions can not be generalized to situations in which full-blown autobiographical pseudo-memories are implanted (Pezdek \& Lam, 2007). There are two issues here that are relevant to the study of pseudomemories. One issue is whether laboratory phenomena like false fame illusions tap a broad aspect of source monitoring integrity. We believe they do: It is not unlikely that people who mistake familiarity for fame also tend to confuse fantasies that feel familiar with memories of things that really happened. A second issue is that of causality. One causal scenario is that individuals with source monitoring difficulties are more sensitive to the memory corrupting effects of hypnosis and develop pseudomemories of previous lives along this route. Another causal scenario is that treatment techniques like hypnosis and memories of previous lives have led to a liberal style in making source monitoring decisions. Of course, our results are silent as to this causal issue.

Several limitations of the current study deserve some comment. Firstly, like most studies on people with alien abductions, our study relied on a small sample. Yet, effect sizes indicate that our findings are quite robust. Secondly, unlike Clancy and her colleagues (e.g., Clancy et al., 2002), we did not differentiate between those who recovered or repressed their memories of previous lives. Another limitation is the lack of control for psychopathology in the previous-life and control group. The participants undergoing previous-life therapy suffered from several psychopathological symptoms. As their scores on the SCL-90 indicate, our control sample did not suffer from psychological distress. Thus, one could speculate that psychopathology is the driving force behind the source monitoring difficulties in the previous-life group. While the non-significant correlation between SCL-90 and false fame illusion seems to argue against this possibility, the point remains that lack of power may have contributed to our failure to detect a significant correlation between these two variables. In line with this, one could argue that differences in psychopathology distress are inherent to our method of recruitment of the previous-life participants. It might well be that persons who undergo reincarnation therapy may be looking for an explanation for their current psychological distress. One possibility in trying to circumvent this problem would be to recruit previous-life participants in the general population or to match the control group on presence of psychopathological signs. In future research, it would also be worthwhile to use three instead of two groups of participants; those who underwent reincarnation therapy and did or did not develop autobiographical memories of previous lives (similar to the recovered and repressed distinction by Clancy et al., 2002), and a control group with no reincarnation beliefs or memories. If both previous-life groups are matched on psychopathology scores, this could also take care of the lack of control for psychopathology that existed in our study. In more general terms, future studies should make an attempt to identify the causal antecedents of source monitoring problems typical for people with implausible memories. 


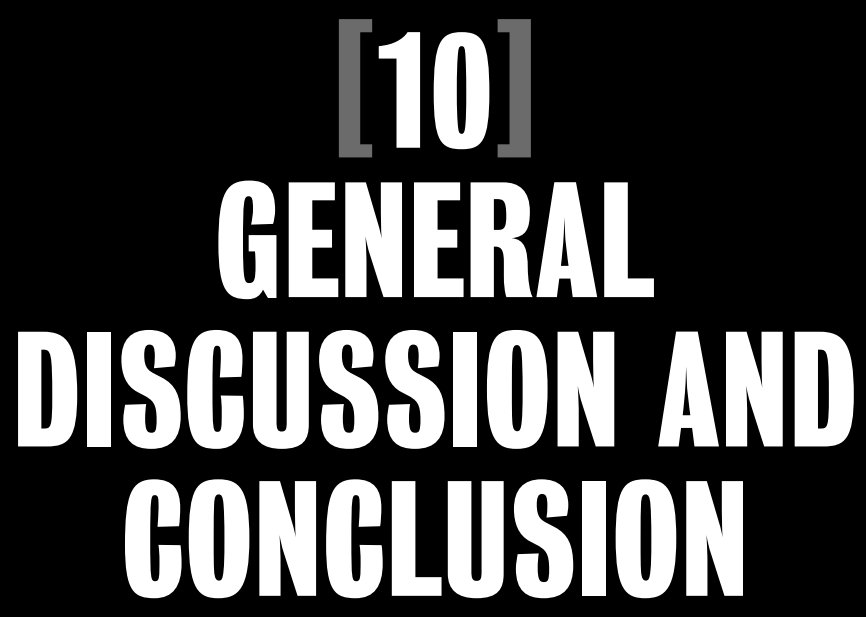





\section{GHAPTER [10] GENERAL DISCUSSION AND CONGLUSION}

The central theme inspiring the studies presented in this dissertation concerned the identification of neurocognitive correlates of source misattributions and pseudomemories in healthy young individuals and in clinical samples. In this Chapter, we will highlight the most important findings of our empirical work. Furthermore, a theoretical framework is presented and directions for future research will be sketched.

\section{Psychometrics and normative data}

Part A of this dissertation focused on the construction, validation, and standardization of a pseudo-memory paradigm and a neurocognitive task. Thus, Chapter 2 described the construction, validation, and standardization of a Dutch version of the Deese/ Roediger-McDermott paradigm (DRM paradigm; Deese, 1959b; Roediger \& McDermott, 1995) in an undergraduate sample. Since most of the studies using this paradigm in foreign (i.e., not English) languages have used translations of the original normative lists (Roediger \& McDermott, 1995; Stadler, Roediger, \& McDermott, 1999), a well-documented Dutch version of the paradigm was in order. Although all lists were constructed in a similar way - namely on the basis of Dutch word association norms - rates of falsely recalling and recognizing the critical lure words varied widely across lists. This can be ascribed to differences in associative strength. Furthermore, participants often recognized critical lures with high confidence, as indicated by the "remember" responses for critical lure intrusions (e.g., Tulving, 1985). Therefore, stimulus material appears to be an important determinant of pseudo-memory rates. Pezdek and Lam (2007) argue that the DRM paradigm is a fail-safe semantic priming task that always produces pseudo-memories. However, we demonstrate in Chapter 2 that this conclusion does not hold, since we identified word lists that did not elicit critical lure pseudo-memories (i.e., null lists). The top 16 lists identified in our study yielded rates of false recall and recognition of critical lures that were comparable to the effects described by Roediger and McDermott (1995) and Stadler, Roediger, and McDermott (1999).

Chapter 3 reports the results of four studies on the factor structure and psychometric properties of the Random Number Generation task (RNG; Ginsburg \& Karpiuk, 1994, 1995), a neurocognitive task measuring monitoring, updating, and inhibition function. More specifically, study 1 revealed a three factor structure solution: seriation, repetition, and cycling. These factors are related to inhibition of stereotypical schemas, output inhibition, and monitoring of previous output, respectively. Study 2 examined the test-retest reliability of these RNG factors in a sample of undergraduate students and schizophrenic patients. We found modest to satisfactory test-retest correlations and no influence of practice, with RNG indices loading on the seriation factor showing statisfactory stability across time. Study 3 and 4 described criterion and construct validity. The results indicated that RNG has acceptable psychometric 
properties and primarily taps inhibition, updating and monitoring functions in normal as well as clinical populations.

\section{Neurocognitive functioning and pseudo-memories}

The aim of the studies described in Part B was to examine the relationships between suboptimal neurocognitive functioning, source misattributions, and pseudo-memory development in healthy undergraduate participants. We specifically focused on inhibitory control (executive function) and working memory capacity, as previous research in older participants and children offered some tentative indication for a specific link between these neurocognitive functions, source misattribution and pseudo-memories (e.g., Alexander, Goodman, Schaaf, Edelstein, Quas, \& Shaver, 2002; Hedden \& Park, 2003; Lödvén, 2003; Mitchell, Johnson, Raye, \& Greene, 2004; Moscovitch, 2000; Reinitz \& Hannigan, 2004; Roberts \& Powell, 2005; Ruffman, Rustin, Garnham, \& Parkin, 2001).

Results from the studies described in this part of the thesis support the idea that suboptimal inhibitory control (either measured directly or manipulated indirectly; see Chapter 7) and working memory capacity are related to recollecting words never presented (Chapters 4 and 5), misattributing imagined actions as performed (Chapter 6), recognizing non-presented stereotype-consistent information, and confabulating information (Chapter 7) in healthy young samples. Chapter 4 showed that in our sample of undergraduates, individual differences in inhibitory function as measured by the RNG task (see Chapter 3) contribute to false recognition and to a lesser extent to false recall. That is, we found a relationship between a deficient inhibition of cognitive schemas and false recognition of non-presented critical lure words in the DRM paradigm. This pattern was further supported by extreme group analysis performed on the inhibition scores. Our findings could not be explained by differences in learning performance between groups. Thus, this study provides evidence for the hypothesized relationship between executive functions and pseudo-memory creation in undergraduate students.

Chapter 5 elaborated on the findings of the previous Chapter. The two studies described in this Chapter investigated whether individual differences in simple and complex Working Memory Capacity (WMC) are related to pseudo-memories of non-presented critical lure words. Given that correct source monitoring is important to avoid pseudo-memory development and working memory is involved in keeping distinctive perceptual and contextual information during encoding and retrieval online, one would have expected an increased susceptibility to pseudo-memories when working memory capacity is suboptimal. We wanted to find out whether this line of reasoning also held for different measures (i.e., aspects) of working memory (simple vs. complex WMC). The two studies described in this Chapter converge on the notion that poor simple WMC (as indexed by suboptimal backward digit span) is related to false recognition and, to a lesser extent false recall of critical lure words. 
Complex WMC did not relate to false recall or recognition of the critical lure words. It should be noted that in our studies, we did not warn participants about the intentions of the DRM paradigm. It is plausible to assume that such warning instruction exerts increased executive demands on WMC, leading to a reduction in encoding distinctive perceptual features. This implies that WMC measures, as they differ in the amount of executive demands that they pose on individuals, have differential effects on source monitoring and pseudo-memory development. Thus, when executive demands are high, complex WMC predicts source monitoring failures. When one has poor complex WMC in a high executive demand environment, encoding of distinctive perceptual and contextual features of to be remembered information is suboptimal. While retrieving this information, distinctive features cannot be used, leading to reliance on more schematic general knowledge (i.e., familiarity). When specific tasks do not require high executive demands like in standard DRM procedures, then simple WMC predicts source monitoring failures.

Since this issue warranted further research, Chapter 6 in part examined whether complex WMC was related to source misattributions in a high executive demand environment. In this study, undergraduates were subjected to an action source monitoring paradigm, in which to-be-remembered acts had to be performed, while others only had to be imagined, thereby putting increased executive demands on WMC. Subsequently, the presented actions were paired with actions that were roughly similar in content and form. For each pair, old-new discrimination (i.e., correct recognition) and source attribution (having performed or only imagined the action) decisions had to be made. In line with the findings of Watson, Bunting, Poole, and Conway (2005) and as concluded in Chapter 5, under increased executive demands (imaging and performing specific action items), poor complex WMC was related to claiming having performed an action while, in fact, it was only imagined. Thus, results of Chapter 6 also indicate that even in a healthy undergraduate sample, WMC, source monitoring, and pseudo-memory development are related in a theoretically meaningful way.

In Chapter 7, we investigated whether an active manipulation of inhibitory control leads to an increased number of source misattributions for general schematic knowledge. More specific, in this study people did or did not receive an instruction to actively inhibit their thoughts on activated neutral or negative stereotypes about race, while reading a stereotype-free crime story. We anticipated that this active inhibition would lead to a decrease in focus on the source of information during encoding due to increased executive demands. During later retrieval, one then more likely relies on primed general schematic knowledge, which may lead to misattributing non-presented schematic knowledge as being presented. This was, indeed, born out by the data. The fact that heightened recognition of non-presented stereotype-consistent information was only found in the negative stereotype prime condition is in line with the stereotype literature. This literature shows that when people ascribe rec- 
ollected items to a stereotyped individual, stereotype-based expectations influence response criteria for making source attributions (e.g., Banaji \& Greenwald, 1995). In this way, perceivers may be more inclined to attribute expectancy-based stereotypical items (e.g., Sherman \& Bessenoff, 1999).

\section{Source monitoring in schizophrenia(-like symptomatology)}

Previous studies have suggested that schizophrenic patients are remarkably adroit at recognizing previously presented verbal information and identifying the source of their memories (e.g., Brébion, Amador, David, Malaspina, Sharif, \& Gorman, 2000; Brébion, Gorman, Amador, Malaspina, \& Sharif, 2002; Brunelin et al., 2006; Keefe, Arnold, Bayen, \& Harvey, 1999; Moritz, Woodward, Cuttler, Whitman, \& Watson, 2004; Moritz, Woodward, \& Ruff, 2003; Nienow \& Docherty, 2004; Vinogradov, Willis-Shore, Poole, Marten, Ober, \& Shenaut, 1997). The antecedents of these recognition errors and source misattributions are, however, open to empirical scrutiny. Two lines of research may elucidate these memory aberrations in schizophrenia.

To begin with, apart from the involvement of medial temporal lobe/diencephalon (MTL/D) functions, memory functioning in schizophrenia may also be affected by executive dysfunctions. Since schizophrenic patients exhibit marked deficits in executive and memory tasks (e.g., McKenna, Ornstein, \& Baddeley, 2002; Weinberger et al., 2001), this hypothesis was investigated in Chapter 8. Results showed that, relative to controls, patients with schizophrenia exhibit a strong tendency to recognize presented (i.e., old) words as new (i.e., misses). Furthermore, correct recognition rates of the schizophrenic patients were significantly lower compared to those of controls (e.g., Moritz, Heeren, Andresen, \& Krausz, 2001b) and were accompanied by a lowered confidence. As to pseudo-memories, in line with prior research (e.g., Elvevåg et al., 2004), both groups showed a substantial number of pseudo-memories for critical lure words. However, it was the control group that had the highest level of false recognition. For source misattribution, our findings concur with previous research by Keefe et al. (1999), Moritz et al., (2003), and Vinogradov et al. (1997) in that schizophrenic patients, compared to healthy controls, were more likely to make internal-external source misattributions with inflated confidence ratings. The executive functioning indexes were significant predictors of correct recognition, but not of source misattributions or pseudo-memories.

Secondly, we investigated whether participants with schizophrenia-like symptomatology show similar memory aberrations as those with schizophrenia, and whether neurocognitive deficits relate to these memory aberrations. The study described in Chapter 6 demonstrates that participants with high scores on the Schizotypal Personality Scale (STA; Claridge \& Broks, 1984) more often falsely claim to have performed actions when in fact they have only imagined them. Furthermore, significant negative correlations were found between STA scores, correct recognition, and source attribution scores, indicating poorer correct recognition and more source mis- 
attributions with increasing levels of schizotypy. However, high and low schizotypal participants did not differ with regard to neurocognitive functions as measured by a complex WMC task (i.e., O-span). These findings are in line with source monitoring studies among schizophrenic patients (e.g., Nienow \& Docherty, 2004) and hallucination-prone participants (Larøi, Collignon, \& Van der Linden, 2005) in that schizotypal participants exhibited a deficiency in internal source attribution.

In both Chapters (6 and 8 ), susceptibility to source misattribution was not related to WMC (Chapter 6) or executive dysfunctions (Chapter 8). Only correct recognition was predicted by executive function measures in the schizophrenic sample. It seems that prefrontal mediated cognitive (dys)functions are only one of several factors that contribute to different memory aberrations in schizophrenia and schizophrenia-like symptomatology. More recently, it has been hypothesized that schizophrenic patients display a more general cognitive deficit, namely a tendency to excessive knowledge corruption. That is, a vast amount of what patients strongly believe to be true is, in fact, false (e.g., Moritz et al., 2003; 2004; Moritz \& Woodward, 2006) and this false information is held with strong conviction. This was also found in Chapter 8 , where greater knowledge corruption in schizophrenia was detected for source misattributions and misses (see also Moritz \& Woodward, 2006). This knowledge corruption could be a consequence of a liberal acceptance bias, whereby fragmented and partial information is taken as sufficient evidence to accept a response option. In this sense, schizophrenic patients increasingly rely on the familiarity of an event, thereby merely knowing that an item has occurred without experiencing vivid perceptual recollections. Absence of such retrieval cues for errors will caution healthy participants against high confidence ratings, whereas participants with a more liberal acceptance threshold will use this information as sufficient evidence for high levels of subjective confidence.

\section{Plausibility, familiarity, and recollection}

Several researchers argue that plausibility is an important prerequisite for source misattribution and pseudo-memory development (e.g., Mazzoni, Loftus, \& Kirsch, 2001; Pezdek, Finger, \& Hodge, 1997). Plausible events fuel familiarity ("It could have happened"). Once familiarity of an event is achieved, this can relatively easily be converted into a belief that the event did take place, with the following step being misattribution into a true recollection. Typical studies to investigate this hypothesis have presented participants with descriptions of true and false plausible and implausible events. After this presentation, it is identified whether source misattributions are more prevalent for plausible as compared to implausible false events or vice versa (Pezdek et al., 1997). A fascinating question that arises is whether reports of implausible events are also associated with a tendency to commit source misattributions. This issue was studied in Chapter 9, in which participants with highly implausible autobiographical beliefs and memories (hypnotically induced previous-life 
beliefs and memories) were compared to matched controls who did not report such highly implausible events. Results showed that previous-life participants were more susceptible to source misattributions in comparison to matched control participants. This can best be explained by the fact that an implausible event can become subjectively plausible once this event is repeatedly imagined or thought about.This, in turn, may create a feeling of familiarity (e.g., "Illusion of Truth"; Arkes, Hackett, \& Boehm, 1989; Bacon, 1979). Familiarity can be erroneously interpreted as a diagnostic sign of the truth status of an event, thus creating a belief that the event indeed has happened. The following step is to misattribute it as a genuine memory. Our findings suggest that subjective rather than objective plausibility may be seen as a necessary factor in the cascade to a full blown pseudo-memory.

We readily acknowledge that in our discussion so far, we have implicitly alluded to causal mechanisms amounting to the idea that prefrontal dysfunctions, source misattributions, familiarity and so forth function as antecedents of pseudo-memories. But, of course, the studies described in this thesis were largely correlational in nature and so there is the theoretical possibility that people first come to adopt pseudo-memories (e.g., as a result of social pressure, e.g., Roediger, Meade, \& Bergman, 2001) and then become sloppy in their source monitoring decisions.

\section{An integrated theoretical framework}

With this restriction in mind, we will briefly describe an adapted memory reconstruction framework, inspired by the findings of this dissertation. This framework also draws upon the ideas from several other investigators, including Johnson et al. (1993), Kopelman (1999, 2002), McDermott and Watson (2001), Moscovitch and Winocor (2002), Reyna and Brainerd (1995), and Schacter et al. (1998). It is not our intention to completely review and reformulate existing theories (as described in Chapter 1). Our objective is to add to the framework that can be derived from these theories the main findings of this dissertation. The resulting framework focuses on the key encoding, consolidation, retrieval processes, and neural correlates that contribute to the reconstructive aspects of memory.

In summarizing the framework described in Chapter 1, memory representations of past events can best be described as patterns of features that were active during encoding of different aspects of the experience (e.g., Johnson et al., 1993). During encoding, these features need to be bound together efficiently, but also need to be distinctive enough (pattern separation). To remember an event, one has to reactivate the features that constitute the desired memory representation and set specific criterions for memory retrieval. During encoding, consolidation, and retrieval, there are several problems that need to be solved in order to create accurate representations of past events. Internal (neurocognitive functions, general schematic knowledge, personal plausibility) and external (social influences) factors can influence these memory processes (see figure 10.1). 


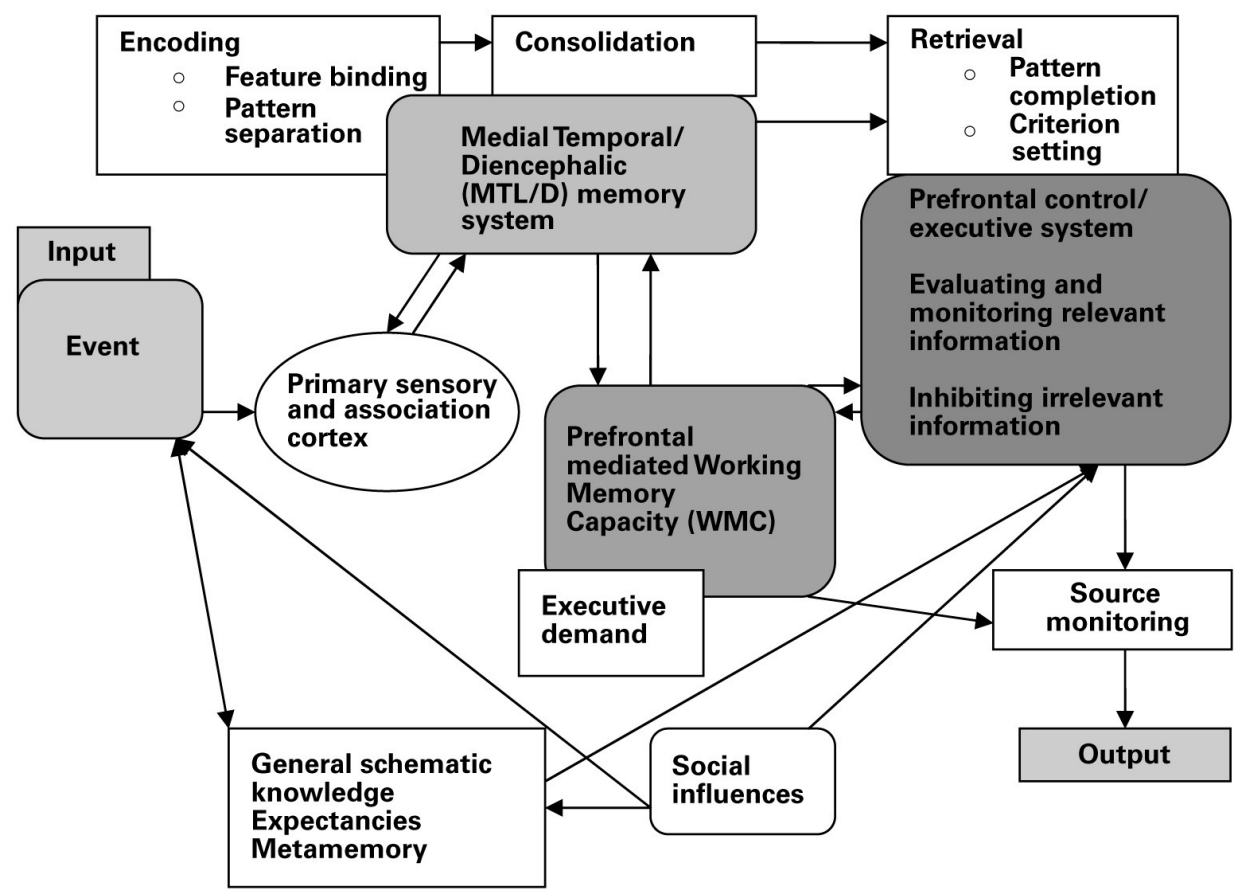

Figure 10.1 Systems inVolved in the generation of PSEUdo-memories.

\section{Encoding and consolidation}

On receiving input (real life event, stimulus material, etc.) that needs to be encoded, perceptual and contextual details making up the memory representation need to be connected to form a "coherent representation" (e.g., feature binding process; Moscovitch, 1994; Moscovitch $\&$ Winocur, 2002). When attention is paid to this input, different regions in the primary sensory and association cortex are activated that constitute the different aspects of this input. The medial temporal lobe/diencephalon (MTL/D) area connects these different brain regions that are simultaneously active during the encoding of this event. Thus, distributed patterns of activity in the neocortex that constitute the memory of different aspects are linked to representations in region CA3 of the hippocampus (Dodson \& Schacter, 2002a), whereby each aspect of the representation is assigned its own hippocampal "index". 
A necessary function for optimal encoding is efficient working memory (e.g., Cowan, 2005). Working memory capacity (WMC) can be described as the set of processes that hold a limited amount of information in a readily accessible state for use in an active task. During encoding, this neurocognitive function is involved in holding memory representations on line, updating these representations, assigning memory for temporal order, selective attending to-be-stored information, and manipulating information. As was found in Chapters 5 and 6, suboptimal WMC undermines feature binding and pattern separation processes. As a consequence, ineffective feature binding leaves the person only with a global impression of the event (i.e., remembering the general gist or familiarity of the event). A second problem that then needs to be solved at encoding is to keep the bound representations separate from each other. If there is overlap between representations, one is inclined to rely on general similarities (i.e., familiarity), which opens the gates for source misattribution and pseudo-memory development. A recent meta-analysis (e.g., Wager \& Smith, 2003) found support for left frontal dominance in verbal working memory capacity tasks with low executive demands (e.g., digit span tasks). The authors also noted that WMC tasks requiring increased executive processing (e.g., complex WMC tasks) generally produce more dorsal frontal activations than do storage-only tasks. This underlies the conclusion drawn in Chapter 5 that WMC tasks differing in executive demands may differentially affect source monitoring. The involvement of different neural processes may thus explain why WMC tasks are not in the same way related to source monitoring. This issue, however, needs to be further investigated.

The MTL/D region also contributes to pattern completion at retrieval. During the retrieval of relatively recent episodes (for which there still is a hippocampal index corresponding to that aspect of an event), cues activate the event's index in region CA3 of the hippocampus, and activation spreads from the index to all the features comprising that event. Once an event is consolidated, however, activation can spread directly between the episode's features, and the hippocampus no longer plays an important role in pattern completion. Thus, although the MTL/D plays an important role in the encoding of memory traces, it does not appear to be a necessary part of the retrieval of remote episodic memories.

\section{Retrieval}

Accurate encoding of memory representations is not sufficient to retrieve an accurate representation. Of importance is the degree to which features of the retrieval cue match the features of the memory representation during pattern completion. One problem is that retrieval cues can potentially match representations other than the one that is desired. This is most certainly the case when representations overlap or when representations are inefficiently encoded, which may lead to overreliance on the general gist or familiarity of representations. For this reason, retrieval has to be focused. 
Once familiarity or recollection-based memory representations are in the focus of attention, a source monitoring decision must be made about whether the activated information is a veridical recollection of a previously experienced event, or whether it is based on fantasy, experiences by others, or memories of imagined events. During this phase of the retrieval process, criterion-based decisions must be made, such as evaluating and monitoring relevant information and inhibiting irrelevant information. The prefrontal cortex (PFC) plays a critical role in this effortful aspect of retrieval. In other words, the PFC contributes "intelligence" to what essentially is a "stupid" MTL/D structure (e.g., Moscovitch \& Winocur, 2002, p. 205). Inefficient criterion setting based on subtle neurocognitive deficits in working memory or executive functioning can relate to source misattributions and later pseudo-memory development, as was suggested by the findings in our studies (described in Chapters 4, 5, 6, and 7). In this respect, Chapter 4 concludes that retrieval focus and criterion-setting involve cognitive inhibition of schema-related material. Lack of cognitive inhibition may lead to liberal criterion setting and an inability to suppress related information, with the potential consequence of source misattributions. Furthermore, it is in this post-retrieval monitoring stage that general schematic knowledge, expectancies, and metamemorial beliefs can influence memory representations, as was found in Chapter 7 (e.g., Dodson \& Schacter, 2001, 2002b).

Neurocognitive dysfunctions are an important but by no means invariable antecedent of source misattribution and faulty retrieval from declarative memory. Biases introduced by social environment (e.g., social stereotypes; Chapter 7) at input and/or the semantic belief system (believing in implausible events; Chapter 9; Liberal acceptance bias; Chapter 8) may also exert their influence on the prefrontal postretrieval control system and declarative memory retrieval. It seems likely that the interaction between social and biological factors is important.

\section{Future directions}

Personality characteristics, neurocognitive functions, and pseudo-memories

As said before, in healthy samples, there are various traits, notably dissociation and depression (or negative affectivity), that seem to predispose to pseudo-memories (e.g., Candel, Merckelbach, \& Kuijpers, 2003; Horselenberg, Merckelbach, van Breukelen, \& Wessel, 2004). On the other hand, the connection between these traits and pseudo-memories is far from robust (e.g., Wright, Startup, \& Mathews, 2005). That is, some studies were unable to find a significant correlation between dissociation and pseudo-memories (Horselenberg, Merckelbach, Muris, Rassin, Sijsenaar, \& Spaan, 2000). Perhaps, then, traits like dissociation and depression serve as antecedents of pseudo-memories to the extent that they are accompanied by subtle disturbances in neurocognitive functions like poor working memory or poor inhibitory control. Thus, the precise connection between dissociation, depression, neurocognitive functions, and pseudo-memory development requires systematic study. Note 
that there is some tentative evidence that dissociative symptoms go along with mild executive dysfunctions. Relying on a sample of forensic patients, Cima, Merckelbach, Klein, Shellbach-Matties, and Kremer (2001) found that high levels of dissociative symptoms were related to poor performance on a "frontal" task (the Behavioural Assessment of Dysexecutive Syndrome; BADS; Wilson, Alderman, Burgess, Emslie, \& Evans, 1996). Likewise, Giesbrecht, Merckelbach, Geraerts, and Smeets (2004; but see Wright \& Osborne, 2005) noted that in a healthy undergraduate sample, dissociative symptoms were linked to certain aspects of the RNG task (cf. supra). With these findings in mind, we believe that research on the associations between traditional personality traits (e.g., dissociation), neurocognitive functions (e.g., cognitive inhibition, working memory), and pseudo-memories might be fruitful.

\section{Neurocognitive functions and pseudo-memory}

In recent years, researchers have formulated more fine graded models for defining neurocognitive functioning (Friedman \& Miyake, 2004; Miyake, Friedman, Emerson, Witzki, Howerter, \& Wager, 2000). Studies have focused on specific executive functions that have been frequently postulated in the literature. Multiple tasks tapping each target function were then selected. Next, the unity or diversity of these executive functions was examined at the level of latent variables, based on factor analyses and structural equation modeling. For example, Miyake et al. (2000) examined the separability of three executive functions in a healthy adult sample: shifting of mental sets, updating and monitoring of working memory representations, and inhibition of prepotent responses. In their attempt to further unravel inhibitory control, Friedman and Miyake (2004) examined the relationships among three inhibition-related functions in an adult population: resistance to distractor interference, resistance to proactive interference, and prepotent response inhibition. In future research, it would be interesting to look at the separate contributions of these different executive subfunctions to source misattribution and pseudo-memory development, so as to be able to formulate more detailed models of neurocognitive functioning and memory reconstruction.

Apart from the involvement of suboptimal executive functioning and WMC in pseudo-memory, there are good indications that another neurocognitive function may also be relevant here, namely the amount of attention that we allocate to any given task. A typical methodology used in the past years is the dual task, in which two competitive tasks have to be performed simultaneously. This methodology has been particularly fruitful in probing the role of attention in accurate memory (e.g., Baddeley, Lewis, Eldridge, \&Thomson, 1984). A key finding from investigating the effects of dual task or divided attention on memory is that during encoding this manipulation leads to a substantial decrease in accurate memory during retrieval. In recent years, researchers have argued that divided attention conditions may also be important to examine the effects of varying attention demands on source monitoring and 
pseudo-memory development (e.g., Pérez-Mata, Read, \& Diges, 2002; Seamon et al., 2003). In these studies, it is hypothesized that dividing attention disrupts perceptual and contextual encoding (e.g., Troyer \& Craik, 2000), leading to a lack of feature binding and pattern separation. Rather than relying on more detailed forms of "recollection", one may under such conditions be more susceptible to familiarity-based source misattributions. All in all, most of the research so far has explored across-population differences like comparing young adults with older participants (e.g., Koutstaal, Schacter, \& Brenner, 2001). This line of research is still evolving, thereby offering opportunities for theoretical as well as practical relevant research. Below, we elaborate on a recently completed study on the effects of divided attention during encoding on the creation of pseudo-memory.

\section{Divided attention intermezzo}

In this study, we investigated whether divided attention and warning instructions in undergraduate students would influence susceptibility to pseudo-memories for nonpresented critical lure words in the DRM paradigm. The research was partly based on the methodology by Watson et al. (2005) investigating the relationship between complex WMC, warning, and pseudo-memory development. The Watson et al. study showed that undergraduates with suboptimal complex WMC recalled more critical lure words than individuals with high WMC when participants were forewarned about the tendency of the associative lists in the DRM paradigm to elicit non-presented critical lure words (e.g., Gallo, Roberts, \& Seamon, 1997). This finding was thought to reflect participants' suboptimal WMC, i.e., poor ability to actively maintain task goals and their difficulty to avoid the seductive power of familiarity. In our study, we hypothesized that dividing attention would reduce attention given to distinctive perceptual and contextual details and reduce possibilities to actively maintain task goals like keeping online the warning instruction, making recall of the critical lure word in the DRM paradigm more likely. Attention was actively manipulated by means of an oddball task. In a typical oddball task (Huettel \& McCarthy, 2004), participants have to identify infrequent "target" stimuli within a series of rapidly presented "standard" stimuli. This experiment used an auditory oddball task in which infrequent high tones had to be identified amongst frequent low tones. A 2 (warning vs. no warning) $x 2$ (oddball vs. no oddball) between-subjects design was used. Mean proportion DRM correct recall and false recall of critical lures served as primary measures. Our sample consisted of 77 undergraduate students (12 men). Mean age of the participants was 19.43 years $(S D=1.35$; Range: $17-24)$. The four conditions did not differ in age or gender distribution.

Participants were randomly assigned to one of the four groups. In condition 1 (standard group, $n=20$ ), participants were subjected to a standard DRM paradigm, including 16 word lists that were visually presented. In condition 2 (unwarned/divided attention group, $n=18$ ), participants simultaneously had an auditory oddball 
task together with the visual presentation of the 16 DRM word lists. In condition 3 (warned group, $n=20$ ), participants received a warning concerning the potential effects of the DRM paradigm before visual presentation of the word lists. Finally, in condition 4 (warned/divided attention group, $n=19$ ), before simultaneously being subjected to the oddball and DRM task, participants first received a warning instruction. Mean proportion scores of DRM indices for the four conditions can be found in TABLE 10.1.

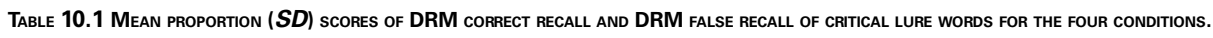

\begin{tabular}{|rcccc|}
\hline & Condition 1 & Condition 2 & Condition 3 & Condition 4 \\
\hline & $M(S D)$ & $M(S D)$ & $M(S D)$ & $M(S D)$ \\
\hline DRM & & & & \\
\hline Correct recall & $.65(.06)$ & $.51(.08)$ & $.63(.08)$ & $.53(.08)$ \\
\hline False recall critical lure & $.30(.14)$ & $.36(.14)$ & $.16(.15)$ & $.34(.19)$ \\
\hline
\end{tabular}

For correct recall, a significant main effect of divided attention was found $[F(1$, 73) $=47.90, p<.001]$ in that participants in the no oddball condition outperformed the participants in the oddball condition. Neither a main effect of warning, nor a significant interaction effect was found (both $F s<2$, both $p^{\prime} s>.05$ ). For recall of critical lure words, significant main effects were found for warning $[F(1,73)=5.04, p<$ $.03]$ and oddball $[F(1,73)=11.79, p=.001]$. More specifically, participants in the no warning condition had more critical lure intrusions compared to those in the warning condition. For the oddball task, simultaneous presentation of an oddball task during word lists significantly increased critical lure intrusions compared to the no-oddball condition. A marginally significant interaction effect was found $[F(1,73)=2.91, p=$ .09], indicating that participants in the no oddball condition with warning made significantly less critical lure intrusions compared to the other conditions.

These new data show that dividing attention can have detrimental effects on correct recall in the DRM paradigm (see for similar results Pérez-Mata et al., 2002; Troyer \& Craik, 2000). Divided attention also significantly increases pseudo-memory development. Moreover, a warning during the oddball task can not reduce pseudo-memory intrusions compared to the oddball task without warning. The effect of warning is in line with findings by Waston, McDermott, and Balota (2004). Meanwhile, research on how divided attention affects pseudo-memory development has primarily focused on word list paradigms. In future research, it would be of theoretical and practical interest to investigate divided attention in other, more ecologically relevant, pseudo-memory paradigms.

Research linking attention to pseudo-memory development has been primarily concerned with actively manipulating attention by presenting dual tasks or giving warnings. However, to the best of our knowledge, no study has looked at how individual differences in attention networks may relate to source monitoring and pseudo-memory development. In the early nineties, Posner and Petersen (1990) proposed 
that sources of attention form a specific system of anatomical areas, which can be broken down into three networks. These three functions involve achieving and maintaining an alert state (alerting), selection of information from sensory input (orienting), and resolving conflicts among responses (executive attention; Fan, McCandliss, Sommer, Raz, \& Posner, 2002). In further elaborating our theoretical framework, it would be of interest to identify the individual contribution of these attention functions to memory reconstruction. For this reason, the attention network test (ANT), specifically designed to measure these three networks could be related to various pseudo-memory paradigms.

\section{Social influence, pseudo-memories, and behavioural consequences}

Most of the studies described in this dissertation bear relevance to specific theoretical issues, in that a more fine graded memory reconstruction model can be described. However, apart from these theoretical issues, relevance to applied fields of psychology is also of interest. In Chapter 7, we argued that primed general schematic knowledge could have detrimental effects on memory accuracy when asked to actively inhibit this general schematic knowledge. Research linking stereotypes to memory accuracy bears relevance to the legal arena. Stereotypes might influence witness reports of criminal events, police interrogations, and court hearings. In fact, it seems safe to assume that all key players in this arena (witnesses, victims, defendants, police investigators, judges, and juries) may be sensitive to the memory undermining effects of stereotypes. A recent study by Lane (2006) is noteworthy in this respect. This study examined the effect of divided attention during encoding of an eyewitness event on participants' memory for the source of post-event misleading information. Results revealed that divided attention participants showed poorer memory for the eyewitness event and were more likely to misattribute post-event misinformation to the event than participants in the full attention condition. Inspired by this finding, we recently completed a study in which we actively manipulated attention during the encoding of an eyewitness event after which post-event stereotype misinformation was given to the participants. Half of the participants thus encoded the crime story, which did not contain any information on the stereotype (i.e., drug addict) under full attention, while the other half was given a divided attention task. After encoding the crime story, participants were given a series of questions about the story and were asked to fill out these questions as if police investigators interrogated them. In each condition, half of the participants were further subjected to a neutral or stereotype misinformation paradigm. The participants in the stereotype misinformation condition received misinformation intended to prime a hard drug addict. In the neutral misinformation condition, non-stereotype items were suggested to the participants. To test memory accuracy for the crime story, participants were subjected to a recognition task, containing factual items, non-witnessed stereotype-neutral items, and non-witnessed stereotype-consistent items. Results showed that participants in 
the divided attention condition scored significantly higher on stereotype-consistent items than participants in the full attention condition. A main effect of misinformation was also found, in that participants in the stereotype misinformation group scored significantly higher on the stereotype-consistent items compared to participants in the neutral misinformation condition. Highest scores on stereotype-consistent items were found for the divided attention group who also received stereotypical misinformation. Even stereotype items that were not part of the misinformation were recognized as such.

These studies show that it might be promising to combine methodologies from different disciplines (e.g., social psychology, experimental psychology, neuropsychology) to approach practically relevant memory reconstruction topics. More specifically, since we found that individual differences in specific neurocognitive functions make people more or less susceptible to source misattributions and pseudo-memories, it would be of interest to identify individuals with low or high WMC, executive functioning, or attention capacity, and to find out whether they are more or less susceptible to social influences like stereotype reliance.

Recently, authors have emphasized the behavioural consequences of source misattributions and pseudo-memories, thus acting upon memory, whether true or false (e.g., Berstein, Laney, Morris, \& Loftus, 2005a,b; Smeets, Merckelbach, Horselenberg, \& Jelicic, 2005). This interest was inspired by the general idea that memory is in the service of overt behaviour (e.g., Neisser, 1996). Cognitive neuroscience has made it plain that, apart from its involvement in memory monitoring, the PFC also employs consolidated memories to direct other activities, such as behavioural planning. Since behavioural consequences are of theoretical and practical importance, it would be interesting to investigate whether, for example, stereotype priming leads to stereotype-consistent pseudo-memories and to stereotype-consistent overt behaviour (e.g., avoiding specific social categories), and whether both effects of stereotype priming are related to each other. Furthermore, it would be of interest to investigate whether specific individuals or environmental conditions (i.e., divided attention) make people more susceptible to act upon true and pseudo-memories.

\section{Cognitive neuroscience and pseudo-memories}

As highlighted in Chapter 1, neuroscience research of memory distortions and pseudo-memories has mainly focused on: 1. Identifying patterns of neural activity that can distinguish between true and false recognition, 2 . Identifying brain regions that contribute to false recognition, and 3 . Identifying which brain regions play a role in monitoring or reducing false recognition (see Schacter \& Slotnick, 2004). There is now general consensus that sensory activity is greater for true than for false recognition and that both the hippocampus and PFC are involved in encoding and retrieving true and pseudo-memories. When overlooking this field of literature, one paradigm stands out as the standard: the DRM paradigm (Deese, 1959b; Roediger \& McDer- 
mott, 1995). This has to do with specific methodological demands: To identify neural activity related to true and pseudo-memories, researchers need to have a substantial number of pseudo-memories per participant to be able to compare them to true ones. Therefore, most of what is known about the cognitive neuroscience of pseudomemories is, in fact, knowledge about the neurobiology of pseudo-memories for words. Okado and Stark (2005) were the first to develop stimulus materials closer to the complex visual scenarios in real life that can elicit memory distortions and can be used in neuroimaging research. These researchers used 8 complex vignettes, each of which was presented to participants through 50 colour slides. Participants were scanned while watching these vignettes (i.e., original event phase). Later, during a standard misinformation phase, participants watched these vignettes again, not being aware that 12 critical slides were altered in some way. Two days later, a recognition task was presented, comprising of multiple choice factual knowledge questions and a source memory test. Activity that subsequently led to true and pseudo-memories was examined during both encoding phases. Results showed two interaction patterns between encoding phase and type of memory in the MTL/D and PFC regions. In the left hippocampus tail and perirhinal cortex, a predictive item-encoding pattern was observed, in that during the original event phase, activity was greater for true than pseudo-memories, whereas during the misinformation phase, activity was greater for pseudo than true memories. A second pattern of activity consistent with encoding source or contextual aspects was observed in the hippocampus and adjacent cortex. It would be informative to explore in future studies whether complex and "realistic" pseudo-memory paradigms like the one used by Okado and Stark (2005), can be implemented in cognitive neuroscience settings, thereby increasing practical use and generalizibility of the standard DRM findings. Furthermore, since every neuroimaging technique (functional Magnetic Resonance Imaging, Positron Emission Tomography, electroencephalogram) has its drawbacks of lacking spatial resolution, temporal resolution, and/or causal interpretations, it would be informative to combine these techniques and to incorporate new tools for memory reconstruction research likeTranscranial Magnetic Stimulation (TMS; Komssi \& Kähkönen, 2006; Sack \& Linden, 2003). TMS can be conceptualized as a virtual lesion technique, capable of disrupting organized cortical activity, transiently and reversibly. Combining neuroimaging techniques, like TMS-EEG or TMS-fMRI would provide us with more detailed insight in cortical reactivity and connectivity underlying memory reconstruction.

\section{Clinical implications}

Our results may have implications for the field of clinical psychology and neuropsychology. To begin with, there are some tentative indications that internal source misattributions may be seen as a risk factor for developing schizophrenia. In line with source monitoring studies in schizophrenia (e.g., Nienow \& Docherty, 2004) and schizophrenia-like symptomatology (e.g., non-clinical positive symptomatology; 
Larøi et al., 2005), schizotypal participants have been found to exhibit a deficiency in internal source attribution. It would be of clinical relevance to set up longitudinal studies in which participants showing schizophrenia-like symptomatology are followed and monitored on source misattributions to determine whether deterioration in source misattribution is associated with the onset of schizophrenia. Source misattribution and in particular a tendency to treat imagined events as having an external origin, may convey a sense of reality to unusual sensations, paranoid ideation, and idiosyncratic beliefs and perceptions. If such internal-external misattributions serve, indeed, as antecedents of schizophrenia, early interventions targeting these misattributions might have therapeutic potential.

More generally, identifying the neurocognitive antecedents of pseudo-memories can help clinicians developing neurocognitive rehabilitation programs for clinical samples. An example would be metacognitive skills trainings for schizophrenic patients. Thus, people who are at increased risk for making source misattributions and pseudo-memories due to neurocognitive deficits may learn to be more aware of this risk, may be cautioned about trusting partial information, and may learn ways to confirm hypotheses in a more stringent manner.

\section{Forensic implications}

The MP3 murder case

On Wednesday April 12 $2^{\text {th }}, 2006$, around 4.15 pm, the 17 year old Joe van Holsbeeck and a friend were awaiting the arrival of another friend in the entrance hall of the central railway station in Brussels, Belgium. Their friend was scheduled to arrive by train at $4.30 \mathrm{pm}$. To pass the time, Joe listened to music on his MP3-player. Then, two young men asked Joe and his friend for directions to the Nieuwstraat, a well-known shopping street in Brussels. A few moments later, their intentions changed as they tried to steal Joe's MP3-player. Joe resisted and one of the offenders drew a knife. The other culprit pushed Joe's friend aside. Joe was stabbed several times, once near his hart. The two offenders fled in the direction of the Grand-Place. Several hours later, Joe succumbed to his injuries in the Sint-Pieters hospital in Brussels.

On Thursday April 13 ${ }^{\text {th }}, 2006$, the police questioned thousands of commuters in the entrance hall of the central station in Brussels. They were asked whether they had witnessed what from then on was called the "MP3 murder".This yielded 20 useful tips. A recurrent statement by several witnesses was that the perpetrators were North-Africans, probably Moroccans. A police spokesman adopted this typology and stated on Belgian news broadcasts that the culprits had a North-African background. It was in this climate that Brussels Member of Parliament, Fouad Ahidar, called on the immigrant community to help searching the perpetrators of this murder. He also stated in the press: "For a long time now, I try to raise this matter with the immigrant community and federal authorities that criminal Moroccan or Turkish youngsters choose their victim on the basis of their looks of being not religious persons." 
At the same time, photographs showing the perpetrators drawn from closed circuit television video footage, picked up by surveillance camera's around the Brussels central station were released by the police authorities. On these somewhat blurred photographs, most of the Belgian people indeed identified the perpetrators as Moroccan orTurkish. However, eyewitness accounts did not reveal any new and useful information. For this reason, the Belgian police authorities decided to narrow their investigation and show video footage of the surveillance camera's to several schools in the Brussels region. This turned out to be the right decision. Several teachers and students identified the perpetrators as Adam G. and Mariusz O. Both perpetrators were eventually arrested and confessed. They were young adolescents and had a Polish background.

This case description shows how general schematic knowledge (i.e., stereotypes) can affect our perception and lead to expectancy based stereotype-consistent pseudo-memories once this general schematic knowledge has been triggered. The effect of general schematic knowledge, like stereotypes, on memory accuracy is underrated in judicial settings. As was found in several studies including ours, priming participants with stereotype knowledge, either based on race, gender, or other social categories may undermine accurate memory. When combined with instructions not to think of specific stereotype information (as can be the case in jury-based courtrooms) or in circumstances where one is distracted by other peripheral actions (dividing attention, as can be the case when witnessing a criminal event), one is inclined to rely on general schematic knowledge thereby blurring the original memory representations.

\section{Neuropsychology in the judicial system}

The two introductory cases (Bob Hunter and JD) describe two brain-damaged patients who came to the attention of police investigators. The review in Chapter 1 and the studies on pseudo-memories in undergraduates described in this dissertation, make it clear that in cases such as those with which we began this dissertation, knowledge about the relationship between neurocognitive functions and memory reconstruction might inform police investigations. Clearly, specific precautions should be taken when interrogating people with brain damage, like asking short questions, limiting the length of the interrogation, avoiding suggestive questioning, and so on. When one does not have this specific knowledge, it may happen that one misinterprets the testimonies of, say, Bob Hunter as accurate recollections of past experiences. This point does not only hold for brain damaged suspects or eyewitnesses. As described in Chapter 8 , specific psychopathology like schizophrenia may also undermine memory accuracy. When these patients come in contact with police investigators, similar precautions are in order (see for example, Merckelbach, Smeets, Peters, \& Jelicic, 2005). Finally, as discussed in this dissertation, individual differences in neurocognitive functions could also influence memory accuracy in children, undergraduates, and the elderly. 
To make professionals sensitive to this specific problem, it should be dealt with in courses on interviewing techniques at the police academy. Perhaps, one should consider screening vulnerable suspects or eyewitnesses on neurological or psychopathological status as soon as they enter the police station. Trained forensic psychologists and neuropsychologists could play an important role in this screening. They could also provide police investigators with specific clues as to how to set up their interrogations in order to receive accurate recollections.

\section{Final remarks}

The data presented in this dissertation demonstrate that individual differences in neurocognitive functions are related to memory reconstruction. More specifically, cognitive functions related to what Goldberg (2001, p. 21) describes as the "brain's chief executive office" clearly contribute to memory reconstruction. This author (Goldberg, 2001, p. 22) further suggests: “The leader's role is elusive but critical. Let the leader lapse, however briefly, and disaster strikes." In drawing parallels with our findings, when the leader does not function optimally, small calamities may occur, like misattributing a fantasy to a real event. In the legal arena, these "small" calamities sometimes have "disastrous" consequences. 


\section{NOTES}

\section{Chapter 1 - General introduction: Neuropsychology and pseudo-memories}

${ }^{1}$ The story of Bob Hunter is also described in Merckelbach \& Jelicic (2005), Hoe een CIA-agent zijn geheugen hervond en andere waargebeurde verhalen (pp. 137-150), and Merckelbach, Jelicic, Candel, \& Horselenberg (2002), Sleutelen aan geheugenverlies: iatrogene elaboratie van een retrograde amnesia, Maandblad Geestelijke Volksgezondheid, 57, 923-935. Also, a documentary was devoted to this case, entitled "De man in het niets" by Rens Oomens and André Bannenberg.

${ }^{2}$ The story of JD is based on a real case. The case has been anonimized and essential demographic details have been changed. See Case No. AY8840, Rechtbank Breda, 26 September 2006, available at : http://rechtspraak.nl.

${ }^{3}$ In this review, we do not address the distinction between episodic and semantic memory. However, it should be noted that there is still no consensus on whether episodic and semantic information is acquired through distinct memory systems and have different neural correlates or whether both kinds of information are acquired through common processes and brain circuits (e.g., Foster \& Jelicic, 1999; Gainotti, 2006; Moscovitch et al., 2005).

\section{Chapter 2 - Inducing pseudo-memories: A Dutch version of the Deese/Roediger-Mc- Dermott paradigm}

${ }^{1}$ In the Stadler et al. (1999) normative study, associations to 36 critical lures were used. For 4 critical lures (flag, high, rubber, slow), no Dutch normative data were available in the Lauteslager et al. (1986), Van Loon - Vervoorn, and van Bekkum, (1991), and van der Made-van Bekkum (1973) Dutch word association norms. These 4 words were therefore not used in the current study.

\section{Chapter 3 - The Random Number Generation task: Psychometric properties and nor- mative data of an executive function task in a mixed sample}

${ }^{1}$ One could speculate that these instructions may influence the RNG outcome measures of repetition avoidance or serial responding. However, several studies (Chapter 4; Towse, 1998) have found that healthy participants who have received these instructions commit qualitatively and quantitatively similar errors as those without such warning (e.g., Giesbrecht, Merckelbach, Geraerts, \& Smeets, 2004; Ginsburg \& Karpiuk, 1994). 
${ }^{2}$ When looking at the underlying correlations between the extracted factors in the direct oblimin PCA, no significant correlations were apparent (all $r$ 's $<.15$ ). This shows that the three extracted factors are independent, thereby supporting the use of Varimax PCA.

${ }^{3}$ Previous research has found that randomization performance in schizophrenia may improve with onset of neuroleptic medication due to an improvement of concentration, but soon declines again to off-medication baseline (e.g., Axmacher, Bente, \& Ferner, 1970).

${ }^{4}$ Here, we describe the standardized factor scores for the test-retest stability and construct validity of the RNG. Results for separate RNG indices can be obtained from the author.

${ }^{5}$ Separate one-way ANOVA's were also carried out with groups being the Study 2 young adult subsample $(n=59)$, the mid-age subsample $(n=40)$, and the schizophrenic subsample $(n=26)$, using post-hoc Bonferroni corrections. These analyses yielded similar results.

6 The Games-Howell post-hoc procedure is designed to analyze data from unbalanced designs in which sample variances differ (e.g., Field, 2005).

Chapter 4 - Recollecting words never presented part I: Mild executive dysfunctions in undergraduate students

${ }^{1}$ Analyses based on factor scores as described in Chapter 3 yielded comparable correlations in that proportion recognition of critical lure words was significantly related to the seriation factor: $r=.26, p=.03$. All other correlations between factor scores and DRM recall and recognition indices remained non-significant.

\section{Chapter 5 - Recollecting words never prestented part II: Poor working memory ca- pacity in undergraduate students}

${ }^{1}$ To correct for possible ceiling effects in our critical lure data, corrected false recognition of critical lures (false recognition of critical lure - false recognition of unrelated lure; $M=.81, S D=.16$; Range: .65) was calculated and related to the digit span scores. Only backward digit span score was negatively related to corrected false recognition of critical lures and in fact the magnitude of this correlation was similar to that found in the uncorrected analysis $(r=-.39, p<.01)$. 
${ }^{2}$ As in Study 1, we also calculated corrected false recognition of critical lures $(M=.63$, $S D=.16$; Range $=.75$ ) to control for possible ceiling effects in false recognition. In line with Study 1 , and the analyses in Study 2, only backward digit span significantly correlated with corrected false recognition $(r=-.50, p<.01)$.

Chapter 6 - Action source monitoring, schizotypal traits, and working memory

No footnotes

Chapter 7 - Suppression of stereotypes leads to false recollections of a crime: Influence of inhibition instruction on memory accuracy

${ }^{1}$ In this pilot study, the order of the primes was counterbalanced. Mean sympathy score for the Dutch group was $6.23(S D=1.08)$ and that for the foreign group was $4.61(S D=1.00)$.

Chapter 8 - Memory efficiency, source attributions, and executive dysfunctions in schizophrenia

${ }^{1}$ WCST categories completed was left out of the regression analyses, because this variable strongly correlated with the other WCST scores $(r>.80, p<.001)$, indicating multicollinearity.

${ }^{2}$ A negative correlation was found between misses and false alarms of critical lure words when both samples were pooled $(r=-.49, p<.001$; two-tailed) thereby underscoring this possible explanation.

\section{Chapter 9 - Remembering previous lives and source monitoring}

${ }^{1}$ Both types of correlational analyses (collapsing across groups and groups separately) were also carried out using non-parametric statistics (Spearman's rho). All correlations between false fame index and personality traits remained non-significant, all $r_{s}^{\prime} s<.40$, all $p^{\prime} s>.05$.

\section{Chapter 10 - General discussion and conclusion}

No footnotes 



\section{[R]EEERENGES}

Aleman, A., Hijman, R., de Haan, E.H.F., \& Kahn, R.S. (1999). Memory impairment in schizophrenia: A meta-analysis. American Journal of Psychiatry, 156, 1358-1366.

Alexander, K.W., Goodman, G.S., Schaaf, J.M., Edelstein, R.S., Quas, J.A., \& Shaver, Ph.R. (2002).The role of attachment and cognitive inhibition in children's memory and suggestibility for a stressful event. Journal of Experimental Child Psychology, 83, 262-290.

Allan, K., Dolan, R.J., Fletcher, P.C., \& Rugg, M.D. (2000). The role of the right anterior prefrontal cortex in episodic retrieval. Neurolmage, 11, 217-227.

American Psychiatric Association. (1994). Diagnostic and Statistical Manual of Mental Disorders (4 ${ }^{\text {th }}$ ed.). Washington, DC: American Psychiatric Association Press.

Anastasi, J.S., De Leon, A., \& Rhodes, M.G. (205). Normative data for semantically associated Spanish word lists that create false memories. Behavior Research Methods, 37, 631-637.

Anastasi, A., \& Urbina, S. (1997). Psychological testing, $7^{\text {th }}$ eD. Englewood Cliffs, NJ: Prentice Hall.

Anderson, R.E. (1984). Did I do it or did I only imagine doing it? Journal of Experimental Psychology: General, $113,594-613$

Anderson, M.C., \& Spellman, B.A. (1995). On the status of inhibitory mechanisms in cognition: Memory retrieval as a model case. Psychological Review, 102, 68-100.

Araya, T., Ekehammar, B., \& Akrami, N. (2003). Remembering things that never occurred:The effects of to-be-forgotten stereotypical information. Experimental Psychology, 50, 27-32.

Arkes, H.R., Hackett, C., \& Boehm, L. (1989). The generality of the relation between familiarity and judged validity. Journal of Behavioral Decision MaKing, 2, 81-94.

Artiges, E., Salamé, P., Recasens, C., Poline, J.B., Attar-Levy, D., De la Raillière, A., et al. (2000). Working memory control in patients with schizophrenia: A PET study during a random number generation task. American Journal of Psychiatry, 157, 1517-1519.

Axmacher, I., Bente, D., \& Ferner, U. (1970). Informationsstatistische Untersuchungen zur Struktur einfacher Handlungsfolgen bei endogenen Psychosen [Statistical examiantion of the structure of simple action sequences in endogene psychosis]. ArzneImittel-Forschung [Drugs TREATMENT Research], 20, 919-921.

Bacon, F.T. (1979). Credibility of repeated statements: Memory for trivia. JouRNAL of EXPERIMENTAL PsYCHOLOGY: Human Learning and Memory, 5, 241-252.

Baddeley, A.D. (1966). The capacity for generating information by randomization. QuARTERLY JourNal of Experimental Psychology, 18, 119-129.

Baddeley, A.D. (1986). Working MEMORY. Oxford: Oxford University Press.

Baddeley, A.D. (1996). The fractionation of working memory. Proceedings of the National Academy of Sciences of the United States of America, 93, 13468-13472.

Baddeley, A.D. (1998). The central executive: A concept and some misconceptions. JourNaL of the international Neuropsychological Society, 4, 523-526.

Baddeley, A.D., Emslie, H., Kolodny, J., Duncan, J. (1998). Random generation and executive control of working memory. The Quarterly Journal of Experimental Psychology, 51, 819-852.

Baddeley, A.D., \& Hitch, G. (1974). Working memory. In G.A. Bower (Ed.), The Psychology of LeARNING and motivation. Vol. 8. (pp. 47-89). Hillsdale, NJ: Erlbaum.

Baddeley, A.D., Lewis, V., Eldridge, M., \& Thomson, N. (1984). Attention and retrieval from long-term memory. Journal of Experimental Psychology: General, 13, 518-540.

Balota, D.A., Cortese, M.J., Duchek, J.M., Adams, D., Roediger, H.L.III., McDermott, K.B., et al. (1999). Veridical and false memories in healthy older adults and in dementia of the Alzheimer's type. Cognitive Neuropsychology, 16, 361-384.

Banaji, M.R., \& Greenwald, A.G. (1995). Implicit gender stereotyping in judgments of fame. Journal of Personality and Social Psychology, 68, 181-198.

Bartlett, F. C. (1932). Remembering. Cambridge, UK: Cambridge University Press.

Begg, I., Anas, A., \& Farinacci, S. (1992). Dissociation of processes in belief: Source recollection, statement familiarity, and the illusion of truth. Journal of Experimental Psychology: General, 121, 446458.

Benjamin, A.S. (2001). On the dual effects of repetition on false recognition. JourNaL of EXPERIMENTAL Psychology: Learning, Memory, and Cognition, 27, 941-947.

Bentall, R.P., Claridge, G., \& Slade, P.D. (1989). The multi-dimensional nature of schizotypal traits: A factor analytic study wit normal subjects. British Journal of Clinical Psychology, 28, 363-375.

Beringer, J. (1996). EXPERIMENTAL RUN TIME system (ERTS); Version 3.18. Frankfurt am Main, Germany: Berisoft Corporation.

Bernstein, D.M., Laney, C., Morris, E.K., \& Loftus, E.F. (2005a). False memories about food can lead to food avoidance. Social Cognition, 23, 11-34.

Bernstein, D.M., Laney, C., Morris, E.K., \& Loftus, E.F. (2005b). False beliefs about fattening foods can have healthy consequences. Proceedins of the National Academy of Sciences of the United States, 102, 13724-13731.

Bernstein, E.M., \& Putnam, F.W. (1986). Development, reliability, and validity of a dissociation scale. Journal of Nervous and Mental Disease, 174, 727-735. 
Bradshaw, J.L., \& Mattingley, J.B. (1995). Clinical neuropsychology: Behavioral and brain science. San Diego: Academic Press.

Brainerd, C.J., \& Reyna, V.F. (2001). Fuzzy-trace theory: Dual processes in memory, reasoning, and cognitive neuroscience. Advances in Child Development and Behavior, 28, 41-100.

Brébion, G., Amador, X., David, A., Malaspina, D., Sharif, Z., \& Gorman, J.M. (2000). Positive symptomatology and source monitoring failure in schizophrenia: An analysis of symptom-specific effects. PsYCHIATRY RESEARCH, 95, 119-131.

Brébion, G., Gorman, J.M., Amador, X., Malaspina, D., \& Sharif, Z. (2002). Source monitoring impairments in schizophrenia: Characterization and associations with positive and negative symptomatology. Psychiatry Research, 112, 27-39.

Brébion, G., Gorman, J.M., Malaspina, D., \& Amador, X. (2005). A model of verbal memory impairments in schizophrenia: Two systems and their associations with underlying cognitive processes and clinical symptoms. Psychological Medicine, 35, 133-142.

Brédart, S. (2000). When false memories do not occur: Not thinking of the lure or remembering that it was not heard? MemorY, 8, 123-128.

Broadbent, D.E., Cooper, P.F., Fitzgerald, P., \& Parkes, K.R. (1982). The Cognitive Failure Questionnaire (CFO) and its correlates. British Journal of Clinical Psychology, 21, 1-16.

Brown, R.G., Soliveri, P., \& Jahanshahi, M. (1998). Executive processes in Parkinson's disease: Random number generation and response suppression. NeUROPSYCHOLOGIA, 36, 1355-1362.

Bruce, D., \& Winograd, E. (1998). Remembering Deese's 1959 articles: The Zeitgeist, the sociology of sciences, and false memories. Psychonomic Bulletin and Review, 5, 615-624.

Brugger, P. (1997). Variables that influence the generation of random sequences: An update. Perceptual AND Motor SKILLs, 84, 627-661.

Brugger, P., Monsch, A.U., Salmon, D.P., \& Butters, N. (1996). Random number generation in dementia of the Alzheimer type: A test of frontal executive functions. NeUROPSYCHOLoGIA, 34, 97-103.

Brugger, P., Pietzsch, S., Weidmann, G., Biro, P., \& Alon, E. (1995). Stroop-type interference in random number generation. Psychological Reports, 77, 387-390.

Brunelin, J., Combris, M., Poulet, E., Kallel, L., D'Amato, T., Dalery, J., et al. (2006). Source monitoring deficits in hallucinating compared to non-hallucinating patients with schizophrenia. EUROPEAN Psychiatry, 21, 259-261.

Budson, A.E., Daffner, K.R., Desikan, R., \& Schacter, D.L. (2000). When false recognition is unopposed by true recognition: Gist-based memory distortions in Alzheimer's disease. Neuropsychologr, 14, 277-287.

Budson, A.E., Sullivan, A.L., Daffner, K.R., \& Schacter, D.L. (2003). Semantic versus phonological false recognition in aging and Alzheimer's disease. Brain and Cognition, 51, 251-261.

Budson, A.E., Todman, R.W., \& Schacter, D.L. (2006). Gist memory in Alzheimer's disease: Evidence from categorized pictures. NeUROPSYCHOLOGY, 20, 113-122.

Burnham, W.H. (1889). Memory, historically and experimentally considered. IV. AmERICAN Journal of Psychology, 2, 568-622.

Butler, K.M., McDaniel, M.A., Donburg, C.C., Roediger, H.L.III, \& Price, A.L. (2004). Age differences in veridical and false recall are not inevitable:The role of frontal lobe function. PSYCHONomic Bulletin AND REVIEW, 11, 921-925.

Caltagirone, C., Zoccolotti, P., Originale, G., Daniele, A., \& Mammucari, A. (1989) Autonomic reactivity and facial expression of emotion in brain-damaged patients. In G. Gainotti, \& C. Caltagirone (Eds.), EMOTIONS AND THE DUAL BRAIN (pp. 204-221). Berlin: Springer Verlag.

Candel, I., Merckelbach, H, Jelicic, M., \& Jentjens, K. (2006). Commission errors but not critical lures decrease when you have to pay a price for them. AMERICAN Journal of PSYCHOLogy, 119, 619-624.

Candel, I., Merckelbach, H., \& Kuijpers, M. (2003). Dissociative experiences are related to commissions in emotional memory. BeHAVIOUR RESEARCH AND THERAPY, 41, 719-725.

Ceci, S. J., \& Bruck, M. (1993). Suggestibility of the child witness: A historical review and synthesis. Psychological Bulletin, 113, 403-439.

Cima, M., Merckelbach, H., Klein, B., Shellbach-Matties, R., \& Kremer, K. (2001). Frontal lobe dysfunction, dissociation, and trauma self-reports in forensic psychiatric patients. Journal of NeRVOus and MeNTAL DISEASE, 189, 188-190.

Clancy, S.A., McNally, R.J., Schacter, D.L., Lenzenweger, M.F., \& Pitman, R.K. (2002). Memory distortion in people reporting abduction by aliens. Journal of ABNormal Psychology, 111, 455-461.

Clancy, S.A., Schacter, D.L., McNally, R.J., \& Pitman, R.K. (2000). False recognition in women reporting recovered memories of sexual abuse. Psychological SCIENCE, 11, 26-31.

Claridge, G.S. (1997). SCHIzotyPY: IMPLICATIONS For ILLNESS AND HEALTH. Oxford: Oxford University Press.

Claridge, G.S., \& Broks, P. (1984). Schizotypy and hemisphere function. I: Theoretical considerations and the measurement of schizotypy. Personality and Individual Differences, 5, 633-648.

Cohen, G., \& Faulkner, D. (1989). Age differences in source forgetting: Effects on reality monitoring and on eyewitness testimony. Psychology \& AGING, 4, 10-17.

Conway, A.R.A., Kane, M.J., Bunting, M.F., Hambrick, D.Z., Wilhelm, O., \& Engle, R.W. (2005). Working memory span tasks: A methodological review and user's guide. Psychonomic Bulletin and Review, 12, 769-786. 
Cook, A., Arndt, J., \& Lieberman, J.D. (2004). Firing back at the backfire effect:The influence of mortality salience and nullification beliefs on reactions to inadmissible evidence. Law and Human Behavior, 28, 389-410.

Corwin, J. (1994). On measuring discrimination and response bias: Unequal numbers of targets and distractors and two classes of distractors. NEUROPSYCHOLOGY, 8, 110-117.

Cowan, N. (2005). Working MEMORY CAPACITY. New York: Psychology Press.

Crombag, H.F.M., Wagenaar, W.A., \& van Koppen, P.J. (1996). Crashing memories and the problem of "source monitoring". Appled Cognitive Psychology, 10, 95-104.

Curran, T., Schacter, D.L., Norman, K.A., \& Gallucio, L. (1997). False recognition after a right frontal lobe infarction: Memory for general and specific information. NeUROPSYCHOLOGIA, 35, 1035-1047.

Dalla Barba, G. (1993). Confabulation: Knowledge and recollective experience. Cognitive Neuropsychology, 10, 1-20.

Dalla Barba, G., Nedjam, Z., \& Dubois, B. (1999). Confabulation, executive functions, and source memory in Alzheimer's disease. Cognitive Neuropsychology, 27, 1-16.

Daniels, C., Witt, K., Wolff, S., Jansen, O., \& Deuschl, G. (2003). Rate dependency of the human cortical network subserving executive functions during generation of random number series: A functional magnetic resonance imaging study. NeURoscience LeTters, 345, 25-28.

Deese, J. (1959a). Influence of interitem associative strength upon immediate free recall. Psychological REPORTS, 5, 235-241.

Deese, J. (1959b). On the prediction of occurence of particular verbal intrusions in immediate recall. Journal of Experimental Psychology, 58, 17-22.

Dehon, H., \& Brédart, S. (2004). False memories: Young and older adults think of semantic associates at the same rate but young adults are more successful at source monitoring. Psychologr AND AGING, 19, 191-197.

Delbecq-Derousne, J., Beauvois, M.F., \& Shallice,T. (1990). Preserved recall versus impaired recognition. BraIN, 113, 1045-1074.

Derogatis, L.R., Richels, K., \& Rock, A.F. (1976). The SCL-90 and the MMPI: A step in the validation of a new scale. British Journal of PSYCHIATRY, 4, 4280-4289.

D’Esposito, M., \& Postle, B.R. (2002). The neural basis of working memory storage, rehearsal, and

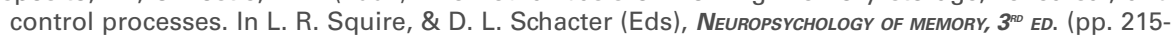
224). NewYork:The Guilford Press.

De Zubicaray, G.I., Smith, G.A., Chalk, J.B., \& Semple, J. (1998). The modified card sorting test: Testretest stability and relationships with demographic variables in a healthy older adult sample. British Journal of Clinical Psychology, 37, 457-466.

Dodson, C.S., \& Schacter, D.L., (2001). "If I had said it I would have remembered it": Reducing false memories with a distinctiveness heuristic. Psychonomic Bulletin ANd ReVIew, 8, 155-161.

Dodson, C. S., \& Schacter, D. L. (2002a). The cognitive neuropsychology of false memories:Theory and data. In A. D. Baddeley, M. D. Kopelman, \& B. A. Wilson (Eds.), HANDBooK OF MEMORY DISORDERS, $3^{\text {ro }}$ ED. (pp. 343-362). Chichester: Wiley \& Sons, Ltd.

Dodson, C.S., \& Schacter, D.L. (2002b). When false recognition meets metacognition:The distinctiveness heuristic. Journal of Memory and Language, 46, 782-803.

Draaisma, D. (2000). Metaphors of memory: A history of ideas about the mind. Cambridge, UK: Cambridge University Press.

Dywan, J., \& Jacoby, L.L. (1990). Effects of aging on source monitoring: Differences in susceptibility to false fame. Psychology \& AgING, 5, 379-387.

Eisen, M.L., \& Lynn, S.J. (2001). Dissociation, memory, and suggestibility in adults and children. APPLIED Cognitive Psychology, 15, S49-S73.

Elvevåg, B., Fisher, J.E., Weickert, T.W., Weinberger, D.R., \& Goldberg, T.E. (2004). Lack of false recognition in schizophrenia: A consequence of poor memory? NEUROPSYCHOLOGIA, 42, 546-554.

Engle, R.W., Cantor, J., \& Carullo, J.J. (1992). Individual differences in working memory and comprehension: A test of four hypotheses. Journal of Experimental Psychology: Learning, Memory, and Cognition, 18, 972-992.

European Monitoring Centre on Racism and Xenophobia. (2005). Majorities' attitudes toward Minorities: Key findings from the Eurobarometer and the European Social Survey. Wien: Manz Crossmedia GmbH \& Co KG. Retrieved June 02, 2005, from http://eumc.eu.int/eumc/material/pub/eurobarometer/EB2005/ EB2005-summary.pdf

Fan, J., McCandliss, B.D., Sommer, T., Raz, A., \& Posner, M.I. (2002). Testing the efficiency and independence of attentional networks. Journal of Cognitive Neuroscience, 14, 340-347.

Field, A. (2005). Discovering statistics using SPSS ,2 $2^{\text {No }}$ ED. London: Sage Publications.

Fletcher, P.C., \& Henson, R.N.A. (2001). Frontal lobes and human memory: Insights from functional neuroimaging. BRAIN, 124, 849-881.

Fletcher, P.C., Shallice, T., Frith, C.D., Frackowiak, R.S., \& Dolan, R.J. (1998). The functional roles of the prefrontal cortex in episodic memory. II. Retrieval. BRAIN, 121, 1249-1256.

Foster, J.K., \& Jelicic, M. (1999). Memory: System, process, or function? Oxford: Oxford University Press. 
Friedman, N.P., \& Miyake, A. (2004). The relations among inhibition and interference control functions: A latent variable analysis. Journal of Experimental Psychology: General, 133, 101-135.

Gainotti, G. (2006). Anatomical functional and cognitive determinants of semantic memory disorders. Neuroscience and Biobehavioral Reviews, 30, 577-594.

Gallo, D.A., McDermott, K.B., Percer, J.M., \& Roediger, H.L.III. (2001). Modality effects in false recall and false recognition. Journal of Experimental Psychology: Learning, Memory, and Cognition, 27, 339-353.

Gallo, D.A., Roberts, M.J., \& Seamon, J.G. (1997). Remembering words not presented in lists: Can we avoid creating false memories? PSYchonomic Bulletin AND REVIEW, 4, 271-276.

Gallo, D.A., \& Roediger, H.L.III. (2002). Variability among word lists in eliciting memory illusions: Evidence for associative activation and monitoring. Journal of MeMORY AND LANGUAGE, 47, 469-497.

Gardiner, J.M. (1988). Functional aspects of recollective experience. Memory and Cognition, 16, 309-313.

Garry, M., Manning, C.G., Loftus, E.F., \& Sherman, S.J. (1996). Imagination inflation: Imagining a childhood event inflates confidence that it occurred. Psychonomic Bulletin and Review, 3, 208-214.

Geraerts, E., Smeets, E., Jelicic, M., van Heerden, J., \& Merckelbach, H (2005). Fantasy proneness, but not self-reported trauma is related to DRM performance of women reporting recovered memories of childhood sexual abuse. Consciousness and Cognition, 14, 602-612.

Gerton, B.K., Brown, T.T., Meyer-Lindenberg, A., Kohn, P., Holt, J.L., Olsen, R.K., et al. (2004). Shared and distinct neurophysiological components of the digits forward and backward tasks as revealed by functional neuroimaging. NeURopsYChOLOGIA, 42, 1781-1787.

Giesbrecht, T., Merckelbach, H., Geraerts, E., \& Smeets, E. (2004). Disruptions in executive functioning and dissociation in undergraduate students. Journal of Nervous and Mental Disease, 192, 567-569.

Ginsburg, N., \& Karpiuk, P. (1994). Random number generation: Analysis of responses. Perceptual and MotoR SKILLS, 79, 1059-1067.

Ginsburg, N., \& Karpiuk, P. (1995). Simulation of human performance on a random generation task. Perceptual and Motor SKILls, 81, 1183-1186.

Goff, L.M., \& Roediger, H.L.III. (1998). Imagination inflation for action events: Repeated imaginings lead to illusory recollections. MEMORY and CogNition, 26, 20-33.

Goldberg, E. (2001). The executive brain: Frontal lobes and the civilized mind. Oxford: Oxford University Press.

Gonsalves, B., \& Paller, K.A. (2002). Mistaken memories: Remembering events that never happened. The NeURosCIENTIST, 8, 391-395.

Goulding, A. (2004). Schizotypy models in relation to subjective health and paranormal beliefs and experiences. Personality and INDIVIDUAL DifFerENCES, 37, 157-167.

Graham, S., \& Lowery, B.S. (2004). Priming unconscious racial stereotypes about adolescent offenders. Law and Human BeHAVIor, 28, 483-504.

Greenberg, D. L., \& Rubin, D. C. (2003). The neuropsychology of autobiographical memory. CoRTEX, 39, 687-728.

Gudjonsson, G.H. (1997). The Gudjonsson Suggestibility Scales Manual. Hove, UK: Psychology Press.

Gudjonsson, G.H., \& Clare, I.C.H. (1995). The relationship between confabulation and intellectual ability, memory, interrogative suggestibility and acquiescence. Personality and Individual Differences, 19, 333-338.

Hagger, C., Buckley, P., Kenny, J.T., Friedman, L., Ubogy, D., \& Meltzer, H.Y. (1993). Improvement in cognitive functions and psychiatric symptoms in treatment-refractory schizophrenic patients receiving clozapine. Biological Psychiatry, 34, 702-712.

Hamilton, D.L., \& Sherman, J.W. (1994). Stereotypes. In R.S. Wryer, Jr. \& T.K. Srull (Eds.), НаNDвоок оF SOCIAL COGNITION (pp. 1-68). Hillsdale, NJ: Erlbaum.

Haraldsson, E. (2003). Children who speak of past-life experiences: Is there a psychological explanation? Psychology and Psychotherapy: Theory, Research and Practice, 76, 55-67.

Hart, R.E., \& Schooler, J.W. (2006). Increasing belief in the experience of an invasive procedure that never happened:The role of plausibility and schematicity. Applied Cognitive Psychology, 20, 661-669.

Hartman, M., Steketee, M.C., Silva, S., Lanning, K., \& Andersson, C. (2003). Wisconsin card sorting test performance in schizophrenia: The role of working memory. SCHIZOPHRENIA RESEARCH, 63, 201-217.

Hasher, L., Goldstein, D., \& Toppino, T. (1977). Frequency and the conference of referential validity. Journal of Verbal Learning and Verbal BehaVior, 16, 107-112.

Heaton, R.K., Chelune, G.J., Talley, J.L., Kay, G.G., \& Curtiss, G. (1993). Wisconsin card sorting test manual: REVISED. Odessa, FL: Psychological Assessment Resources.

Hedden, T., \& Park, D.C. (2003). Contributions of source and inhibitory mechanisms to age-related retroactive interference in verbal working memory. Journal of Experimental Psychology: General, 132, 93-112.

Heinrichs, R.W., \& Zakzanis, K.K. (1998). Neurocognitive deficit in schizophrenia: A quantitative review of the evidence. NeURopsychologr, 12, 426-445.

Henquet, C., Krabbendam, L., Dautzenberg, J., Jolles, J., \& Merckelbach, H. (2005). Confusing thoughts and speech: Source monitoring and psychosis. PsyCHIATRY RESEARCH, 133, 57-63.

Henson, R.N., Shallice, R.J., \& Dolan, R.J. (1999). Right prefrontal cortex and episodic memory retrieval: A functional MRI test of the monitoring hypothesis. BralN, 122, 1367-1381. 
Hertwig, R., Gigerenzer, G., \& Hoffrage, U. (1997).The reiteration effect in hindsight bias. Psychological ReVIew, 104, $194-$ 202.

Hinton, P.R. (2000). Stereotypes, cognition and culture. Hove, UK: Psychology Press.

Horne, R.L., Evans, F.J., \& Orne, M.T. (1982). Random number generation, psychopathology and therapeutic change. ARChives of General Psychiatry, 39, 680-683.

Hornstein, S.L., \& Mulligan, N.W. (2004). Memory for actions: Enactment and source memory. Psychonomic Bulletin and ReVIeW, 11, 367-372.

Horselenberg, R., Merckelbach, H., van Breukelen, G., \& Wessel, I. (2004). Individual differences in the accuracy of autobiographical memory. Clinical Psychology and Psychotherapy, 11, 168-176.

Horselenberg, R., Merckelbach, H., Muris, P., Rassin, E., Sijsenaar, M., \& Spaan, V. (2000). Imagining fictitious childhood events: The role of individual differences in imagination inflation. CLINICAL Psychology AND Psychotherapy, 7, 128-137.

Horselenberg, R., Merckelbach, H., Wessel, I., Verhoeven, C., \& Zeles, G. (2006). Absorption, fantasy proneness and the false fame effect. Personality and Individual Differences, 41, 501-511.

Hudon, C., Belleville, S., Souchay, C., Gély-Nargeot, M.C., Chertkow, H., \& Gauthier, S. (2006). Memory for gist and detail information in Alzheimer's disease and mild cognitive impairment. NeUROPSYCHOLOGY, 20, 566-577.

Huettel, S.A., \& McCarthy, G. (2004). What is odd in the oddball task? Prefrontal cortex is activated by dynamic changes in response strategy. NeUROPSYCHOLOGIA, 42, 379-386.

Hyman, I.E.Jr., \& Kleinknecht, E.E. (1999). False childhood memories: Research, theory, and applications. In L.M. Williams \& V. L.Banyard (Eds.), TRAuma AND memory (pp. 175-188). Thousand Oaks, CA: Sage.

Intons-Peterson, M.J., Rocchi, P., West, T., McLellan, K., \& Hackney, A. (1999). Age, testing at preferred or nonpreferred times (testing optimality), and false memory. Journal of Experimental Psychology: Learning, Memory, and Cognition, 25, 23-40.

Iverson, G.L., \&Tulsky, D.S. (2003). Detecting malingering on theWAIS-III unusual digit span performance patterns in the normal population and in clinical groups. Archives of Clinical Neuropsychology, 18, 1-9.

Jacoby, L.L. (1991). A process dissociation framework: Separating automatic from intentional uses of memory. Journal of Memory and Language, 30, 513-541.

Jacoby, L.L., Kelley, C., Brown, J., \& Jasechko, J. (1989). Becoming famous overnight: Limits on the ability to avoid unconscious influences of the past. Journal of Personality and Social Psychology, 56, 326-338.

Jahanshahi, M., Profice, P., Brown, R.G., Ridding, M.C., Dirnberger, G., \& Rothwell, J.C. (1998). The effects of transcranial magnetic stimulation over the dorsolateral prefrontal cortex on suppression of habitual counting during random number generation. BRAIN, 121, 1533-1544.

Jahanshahi, M., Saleem,T., Ho, A.K., Dirnberger, G., \& Fuller, R. (2006). Random number generation as an index of controlled processing. NeUROPSYCHOLOGY, 20, 391-399.

Janowsky, J.S., Shimamura, A.P., \& Squire, L.R. (1989). Source memory impairment in patients with frontal lobe lesions. NeURopsychologia, 27, 1043-1056.

Jelicic, M., Henquet, C.E.C., Derix, M.M.A., \& Jolles, J. (2001). Test-retest stability of the behavioural assessment of the dysexecutive syndrome in a sample of psychiatric patients. INTERNATIONAL JouRNAL of NeURosCience, 110, 73-78.

Jelicic, M., Smeets, T., Peters, M.J.V., Candel, I., Horselenberg, R., \& Merckelbach, H. (2006). Assassination of a controversial politician: Remembering details from another non-existent film. Applied Cognitive Psychology, 20, 591-596.

Johansson, M., \& Stenberg, G. (2002). Inducing and reducing false memories: A Swedisch version of the Deese-Roediger-McDermott paradigm. Scandinavian Journal of Psychology, 43, 369-383.

Johnson, M.K. (1992). MEM: Mechanisms of recollection. Journal of Cognitive Neuroscience, 4, 268-280.

Johnson, M.K., Hashtroudi, S., \& Lindsay, D.S. (1993). Source monitoring. Psychological Bulletin, 114, 3-28.

Joppich, G., Däuper, J., Dengler, R., Johannes, S., Rodriguez-Fornells, A., \& Münte, T. F. (2004). Brain potentials index executive functions during random number generation. NeURosCIENCE RESEARCH, 49, 157-164.

Kaiser, H.F. (1960). The application of electronic computers to factor analysis. Educational AND PsYCHOLOGICAL Measurement, 20, 141-151.

Kane, M.J., \& Engle, R.W. (2002). The role of prefrontal cortex in working-memory capacity, executive attention, and general fluid intelligence: An individual differences perspective. PsYchoNomic Bulletin AND REVIEW, 9, 637-671.

Kapur, S., Craik, F.l., Jones, C., Brown, G.M., Houle, S., \& Tulving, E. (1995) Functional role of the prefrontal cortex in retrieval of memories: A PET study. NeURoReport, 6, 1880-1884.

Kassin, S.M., \& Sukel, H. (1997). Coerced confessions and the jury: An experimental test of the "Harmless Error" rule. Law and Human Behavior, 21, 27-46.

Keefe, R.S.E., Arnold, M.C., Bayen, U.J., \& Harvey, P.D. (1999). Source monitoring deficits in patients with schizophrenia: A multinominal modelling analysis. Psychological Medicine, 29, 903-914.

Kensinger, E. A., \& Schacter, D. L. (1999). When true memories suppress false memories: Effects of ageing. Cognitive Neuropsychology, 16, 399-415. 
Kimball, D.R., \& Bjork, R.A. (2002). Influences of intentional and unintentional forgetting on false memories. Journal of Experimental Psychology: General, 131, 116-130.

Komssi, S., \& Kähkönen, S. (2006). The novelty value of the combined use of electroencephalography and transcranial magnetic stimulation for neuroscience research. BRAIN RESEARCH REVIEWS, 52, 183192.

Kopelman, M. D. (1999). Varieties of false memory. Cognitive Neuropsychology, 16, 197-214.

Kopelman, M. D. (2002). Disorders of memory. BRAIN, 125, 2152-2190.

Koriat, A., \& Goldsmith, M. (1996). Monitoring and control processes in the strategic regulation of memory accuracy. Psychological Review, 103, 490-517.

Koriat, A., Goldsmith, M., \& Pansky, A. (2000). Toward a psychology of memory accuracy. AnNual ReVIEW of Psychology, 51, 481-537.

Koutstaal, W., \& Schacter, D.L. (1997). Gist-based false recognition of pictures in older and younger adults. Journal of Memory and Language, 37, 555-583.

Koutstaal, W., Schacter, D.L., \& Brenner, C. (2001). Dual task demands and gist-based false recognition of pictures in younger and older adults. Journal of Memory and LANGUAGE, 44, 399-426.

Krabbendam, L., \& Kalff, A.C. (1998). THE BEHAVIOURAL ASSESSMENT OF THE DYSEXECUTIVE SYNDROME - DUtCH VERSION. Lisse, The Netherlands: Swets \& Zeitlinger.

Krabbendam, L., de Vugt, M.E., Derix, M. M.A., \& Jolles, J. (1999). The behavioural assessment of the dysexecutive syndrome as a tool to assess executive functions in schizophrenia. ClINICAL NeUROPSYCHOLOGIST, 13, 370-375.

Kroll, N.E.A., Knight, R.T., Metcalfe, J., Wolf, E.S., \&Tulving, E. (1996). Cohesion failure as a source of memory illusions. Journal of Memory and Language, 35, 176-196.

Lane, S. M. (2006). Divided attention during a witnessed event increases eyewitnes ssuggestibility. Applied Cognitive Psychology, 20, 199-212.

Lange, R., \& Houran, J. (1998). Delusions of the paranormal: A haunting question of perception. JouRNAL of Nervous and Mental Disease, 186, 637-645.

La Pointe, L.B., \& Engle, R.W. (1990). Simple and complex word spans as a measure of working memory capacity. Journal of Experimental Psychology: Learning, Memory and Cognition, 16, 1118-1133.

Larøi, F., Collignon, O., \& Van der Linden, M. (2005). Source monitoring for actions in hallucination proneness. Cognitive Neuropsychiatry, 10, 105-123.

Launay, G., \& Slade, P.D. (1981). The measurement of hallucinatory predisposition in male and female prisoners. Personality and INDIVIDUAL DifFeRENCES, 2, 221-234.

Lauteslager, M., Schaap,Th., \& Schievels, D. (1986). SChrifteliJKe Woordassociatienormen voor 549 Nederlandse ZELFSTANDIGE NAAMWOORDEN. [WRITTEN WORD ASSOCIATION NORMS For 549 DUtCH NOUNs] Lisse: Swets \& Zeitlinger.

Laws, K.R., \& Bhatt, R. (2005). False memories and delusional ideation in normal healthy subjects. Personality and Individual Differences, 39, 775-781.

Lee, J., \& Park, S. (2005). Working memory impairments in schizophrenia: A meta-analysis. Journal oF Aвnormal Psychology, 114, 599-611.

Lencz, T., Smith, C.W., McLaughlin, D., Auther, A., Nakayama, E., Hovey, L., et al. (2006). Generalized and specific neurocognitive deficits in prodromal schizophrenia. BIological Psychiatry, 59, 863-871.

Lenton, A.P., Blair, I.V., \& Hastie, R. (2001). Illusions of gender: Stereotypes evoke false memories. Journal of Experimental Social Psychology, 37, 3-14.

Lenzenweger, M.F., \& Gold, J.M. (2000). Auditory working memory and verbal recall memory in schizotypy. SCHIzophrenia ReSEARCH, 42, 101-110.

Lezak, M.D., Howieson, D.B., \& Loring, D.W. (2004). Neuropsychological assessment. Oxford: Oxford University Press.

Li, C.S.R. (2004). Do schizophrenia patients make more perseverative than non-perseverative errors on the wisconsin card sorting test? A meta-analytic study. PsyCHIATRY RESEARCH, 129, 179-190.

Lödvén, M. (2003). The episodic memory and inhibition accounts of age related increases in false memories: A consistency check. Journal of Memory and Language, 49, 268-283.

Loftus, E. F. (1993). The reality of repressed memories. American Psychologist, 48, 518-537.

Loftus, E.F. (2003). Our changeable memories: Legal and practical implications. Nature Neuroscience REVIEWs, 4, 231-234.

Loftus, E.F. (2005). Planting misinformation in the human mind: A 30-year investigation of the malleability of memory. Learning and MeMory, 12, 361-366.

Loftus, E. F., Feldman, J., \& Dashiell, R. (1995). The reality of illusory memories. In D. L. Schacter (Ed.), MEMORY DISTORTION: How MINDS, BRAINS AND SOCIETIES RECONSTRUCt THE PAST (pp. 47-68). Cambridge, MA: Harvard University Press.

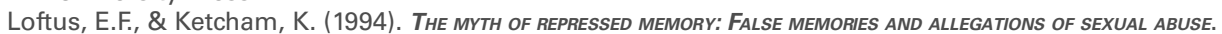
New York: St. Martin's Press.

Loftus, E.F., Miller, D.G., \& Burns, H.J. (1978). Semantic integration of verbal information into a visual memory. Journal of Experimental Psychology: Human Learning and Memory, 4, 19-31.

Loftus, E.F., \& Palmer, J.C. (1974). Reconstruction of automobile destruction: An example of the interaction between language and memory. Journal of Verbal Learning and Verbal Behaviour, 13, 585-589. 
Loftus, E.F., \& Pickrell, J.E. (1995). The formation of false memories. Psychiatric Annals, 25, 720-725.

Macrae, C.N., Bodenhausen, G.V., Milne, A.B., \& Ford, R.L. (1997). On regulation of recollection: The intentional forgetting of stereotypical memories. Journal of PersonaltTy and Social Psychology, 72, 709-719.

Macrae, C.N., Bodenhausen, G.V., Milne, A.B., \& Jetten, J. (1994b). Out of the mind but back in sight: Stereotypes on the rebound. Journal of Personality and Social Psychology, 67, 808-817.

Macrae, C.N., Bodenhausen, G.V., Schloerscheidt, A.M., \& Milne, A.B. (1999). Tales of the unexpected: Executive function and person perception. Journal of Personality and Social Psychology, 76, 200-213.

Macrae, C.N., Milne, A.B., \& Bodenhausen, G.V. (1994a). Stereotypes as energy-saving devices: A peek inside the cognitive toolbox. Journal of Personality and Social Psychology, 66, 37-47.

Macrae, C.N., Schloerscheidt, A.M., Bodenhausen, G.V., \& Milne, A.B. (2002). Creating memory illusions: Expectancy-based processing and the generation of false memories. Mемовт, 10, 63-80.

Made van der-Bekkum van, I.J. (1973). Nederlandse WOordassociatie NORMEN. [DUtCH WORD ASSOCIATION NORMS] Amsterdam: Swets \& Zeitlinger.

Markowitsch, H.J. (1996). Organic and psychogenic retrograde amnesia: Two sides of the same coin? Neurocase, 2, 357-371.

Marsh, E.J., Balota, D.A., \& Roediger, H.L.III. (2005). Learning facts from fiction: The effects of healthy aging and early stage dementia of the Alzheimer's type. Neuropsychologr, 19, 115-129.

Mather, M., Henkel, L.A., \& Johnson, M.K. (1997). Evaluating characteristics of false memories: Remember/know judgments and memory characteristics questionnaire compared. MEMORY AND Cognition, 25, 826-837.

Mather, M., Johnson, M.K., \& De Leonardis, D.M. (1999). Stereotype reliance in source monitoring: Age differences and neuropsychology test correlates. Cognitive Neuropsychology, 16, 437-458.

Mayes, A.R., Downes, J.J., McDonald, C., Rooke, S., Sagar, H.J., \& Meudell, P.R. (1994). Two tests for assessing remote public knowledge: A tool for assessing retrograde amnesia. MEMORY, 2, 183-210.

Mazzoni, G.A., Loftus, E.F., \& Kirsch, I. (2001). Changing beliefs about implausible autobiographical events: A little plausibility goes a long way. Journal of Experimental Psychology: Applied, 7, 51-59.

McCabe, D.P., \& Smith, A.D. (2002). The effect of warnings on false memories in young and older adults. Memory and Cognition, 30, 1065-1077.

McDermott, K.B., \& Watson, J.M. (2001). The rise and fall of false recall: The impact of presentation duration. Journal of Memory and Language, 45, 160-176.

McKenna, P.J., McKay, A.P., \& Laws, K. (2000). Memory in functional psychosis. In G.E. Berrios, and J.R. Hodges (Eds.). Memory disorders in psychiatric practice (pp. 234-267). Cambridge, UK: Cambridge University Press.

McKenna, P.J., Ornstein, T., \& Baddeley, A. (2002). Schizophrenia. In A.D. Baddeley, M.D. Kopelman, and B.A. Wilson (Eds.), НАNDBOOK OF MEMORY disorders, $3^{\text {no }}$ ED. (pp. 413-435). Chichester: Wiley.

McKenna, P.J., Tamlyn, D., Lund, C.E., Mortimer, A.M., Hammond, S., \& Baddeley, A.D. (1990). Amnesic syndrome in schizophrenia. Psychological Medicine, 20, 967-972.

McNally, R.J., \& Clancy, S.A. (2005). Sleep paralysis, sexual abuse, and space alien abduction. Transcultural Psychiatry, 42, 113-122.

McNally, R.J., Lasko, N.B., Clancy, S.A., Macklin, M.L., Pitman, R.K., \& Orr, S.P. (2004). Psychophysiological responding during script-driven imagery in people reporting abduction by space aliens. PSYCHOLOGICAL SCIENCE, 15, 493-497.

Melo, B., Winocur, G., \& Moscovitch, M. (1999). False recall and false recognition: An examination of the effects of selective and combined lesions to the medial temporal lobe/diencephalons and frontal lobe structures. Cognitive Neuropsychology, 16, 343-360.

Merckelbach, H., Horselenberg, R., \& Muris, P. (2001). The Creative Experiences Questionnaire (CEQ): A brief self-report measure of fantasy proneness. PERSONALITY AND INDIVIDUAL DIFFERENCES, 31, 987-995.

Merckelbach, H., Rassin, E., \& Muris, P. (2000). Dissociation, schizotypy, and fantasy proneness in undergraduate students. Journal of Nervous and Mental Disease, 188, 428-431.

Merckelbach, H., Smeets, T., Peters, M.J.V., \& Jelicic, M. (2005). Op weg naar een valse bekentenis: een schizofrene patient wordt verhoord [En route to a false confession: The questioning of schizophrenic suspects]. MaAndblad Geestelijke Volksgezondheid, 60, 827-843.

Merckelbach, H., \& Wessel, I. (1998). Assumptions of students and psychotherapists about memory. Psychological Reports, 82, 763-770.

Miller, M.B., \& Gazzaniga, M.S. (1998). Creating false memories for visual scenes. NeuropsychologiA, 36, 513-520.

Mitchell, J.P., Cloutier, J., Banaji, M.R., \& Macrae, C.N. (2006). Medial prefrontal dissociations during processing of trait diagnostic and nondiagnostic person information. SCAN, 1, 49-55.

Mitchell, J.P., Dodson, C.S., \& Schacter, D.L. (2005). fMRI evidence for the role of recollection in suppressing misattribution errors: The illusory truth effect. Journal of Cognitive Neuroscience, 17, 80-810.

Mitchell, K.J., Johnson, M.K., Raye, C.L., \& Greene, E.J. (2004). Prefrontal cortex activity associated with source monitoring in a working memory task. Journal of Cognitive Neuroscience, 16, 921-934.

Mitchell, J.P., Sullivan, A.L., Schacter, D.L., \& Budson, A.E. (2006). Misattribution errors in Alzheimer's disease:The illusory truth effect. Neuropsychology, 20, 185-192. 
Miyake, A., Friedman, N.P., Emerson, M.J., Witzki, A.H., Howerter, A., \& Wager, T.D. (2000). The unity and diversity of executive functions and their contributions to complex "frontal lobe" tasks: A latent variable analysis. Cognitive Psychology, 41, 49-100.

Monteith, M.J., Ashburn-Nardo, L., Voils, C.I., \& Czopp, A.M. (2002). Putting the brakes on prejudice: On the development and operation of cues for control. Journal of Personality and Social Psychology, 83, 1029-1050.

Monteith, M.J., Sherman, J.W., \& Devine, P.G. (1998a). Suppression as a stereotype control strategy. Personality and Social Psychology Review, 2, 63-82.

Monteith, M.J., Spicer, C.V., \& Tooman, G.D. (1998b). Consequences of stereotype suppression: Stereotypes on and not on the rebound. Journal of Experimental Social Psychology, 34, 355-377.

Moritz, S., Andresen, B., Jacobsen, D., Mersmann, K., Wilke, U., Lambert, M., et al. (2001a). Neuropsychological correlates of schizophrenic syndromes in patients treated with atypical neuroleptics. European Psychiatry, 16, 354-361.

Moritz, S., Heeren, D., Andresen, B., \& Krausz, M. (2001b). An analysis of the specificity and the syndromal correlates of verbal memory impairments in schizophrenia. PSYCHIATRY RESEARCH, 101, 23-31.

Moritz, S., \& Mass, R. (1997). Reduced cognitive inhibition in schizotypy. British Journal of Clinical Psychologr, 36, 365-376.

Moritz, S., \& Woodward, T.S. (2002). Memory confidence and false memories in schizophrenia. JouRNAL of Nervous and Mental Disease, 190, 641-643.

Moritz, S., \& Woodward,T.S. (2006). The contribution of metamemory deficits to schizophrenia. JouRNAL of Abnormal Psychology, 15, 15-25.

Moritz, S., Woodward, T.S., Cuttler, C., Whitman, J.C., \& Watson, J.M. (2004). False memories in schizophrenia. NeURopsychology, 18, 276-283.

Moritz, S., Woodward, T.S., \& Ruff, C.C. (2003). Source monitoring and memory confidence in schizophrenia. Psychological Medicine, 33, 131-139.

Mortimer, A.M. (1997). Cognitive function in schizophrenia: Do neuroleptics make a difference? Pharmacology, Biochemistry and Behavior, 56, 789-795.

Moscovitch, M. (1994). Memory and working-with-memory: Evaluation of a component process model and comparisons with other models. In D.L. Schacter, \& E. Tulving (Eds.), Memory systems 1994 (pp. 311-350). Cambridge, MA: MIT Press.

Moscovitch, M. (2000). Theories of memory and consciousness. In E. Tulving, \& F.I.M. Craik (Eds.), THE OXFORD HANDBOок OF MEMORY (pp. 609-625). New York: Oxford University Press.

Moscovitch, M., Rosenbaum, R.S., Gilboa, A., Addis, D.R., Westmacott, R., Grady, C., et al. (2005). Functional neuroanatomy of remote episodic, semantic, and spatial memory: A unified account based on multiple trace theory. Journal of ANatomy, 207, 35-66.

Moscovitch, M., \&Winocur, G. (2002). The frontal cortex and working with memory. In D.T. Stuss, \& R.T. Knight (Eds.), Principles of frontal lobe function (pp. 188-209). New York: Oxford University Press.

Multhaup, K.S., De Leonardis, D.M., \& Johnson, M.K. (1999). Source memory and eyewitness suggestibility in older adults. Journal of General Psychology, 126, 74-84.

Muris, P., \& Merckelbach, H. (2003). Thought-action fusion and schizotypy in undergraduate students. British Journal of Clinical Psychology, 42, 211-216.

Neisser, U. (1967). Cognitive Psychology. New York: Appleton-Century-Crofts.

Neisser, U. (1996). Remembering as doing. Behavioral and BraIN SCIENCES, 19, 203-204.

Nelson, H.E. (1982). The national adult REAding test. Windsor, UK: NFER-Nelson.

Neuschatz, J.S., Benoit, G.E., \& Payne, D.G. (2003). Effective warnings in the Deese-RoedigerMcDermott false-memory paradigm: The role of identifiability. Journal of Experimental Psychology: Learning, Memory, and Cognition, 29, 35-41.

Nienow, T.M., \& Docherty, N. (2004). Internal source monitoring and thought disorder in schizophrenia. Journal of Nervous and Mental Disease, 192, 696-700.

Norman, K.A., \& Schacter, D.L. (1997). False recognition in young and older adults: Exploring the characteristics of illusory memory. MeMORY and CogNition, 25, 838-848.

Nyberg, L., Cabeza, R., \& Tulving, E. (1996). PET studies on encoding and retrieval: The HERA model. Psychonomic Bulletin and Review, 3, 135-148.

Oberauer, K., Süß, H.M., Schulze, R., Wilhelm, O., \& Wittmann, W.W. (2000). Working memory capacity: Facets of a cognitive ability construct. Personality and Individual DifFerences, 29, 1017-1045.

Okado, Y., \& Stark, C.E.L. (2005). Neural activity during encoding predicts false memories created by misinformation. LEARNING AND MEMORY, 12, 3-11.

Ost, J., Vrij, A., Costall, A., \& Bull, R. (2002). Crashing memories and reality monitoring: Distinguishing between perceptions, imaginations, and 'false memories'. Applied Cognitive Psychologr, 16, 125-134.

Overall, J.E., \& Gorham, D.R. (1988). Brief Psychiatric Rating Scale (BPRS): Recent developments in ascertainment and scaling. Psychopharmacology Bulletin, 24, 97-99.

Parkin, A.J. (1997). The neuropsychology of false memory. Learining and Individual Differences, 9, 341-357.

Parkin, A.J., Bindschaedler, C., Harsent, L., \& Metzler, C. (1996). Pathological false alarm rates following damage to the left frontal cortex. Brain and Cognition, 32, 14-27. 
Parkin, A.J., Leng, N.R.C., Stanhope, H., \& Smith, A.L. (1988). Memory impairment following ruptured aneurysm of the anterior communicating artery. BRAIN AND COGNITION, 7, 231-243.

Parks, T.E. (1997). False memories of having said the unsaid: Some new demonstrations. Applied Cognitive Psychology, 11, 485-494.

Payne, B.K. (2005). Conceptualizing control in social cognition: How executive functioning modulates the expression of automatic stereotyping. Journal of Personality and Social Psychology, 89, 488-503.

Pérez-Mata, M.N., Read, J.D., \& Diges, M. (2002). Effects of divided attention and word concreteness on correct recall and false memory reports. MEMORY, 10, 161-177.

Pezdek, K., Finger, K., \& Hodge, D. (1997). Planting false childhood memories: The role of event plausibility. Psychological SCIENCE, 8, 437-441.

Pezdek, K., \& Lam, S. (2007). What research paradigms have cognitive psychologists used to study "false memory" and what are the implications of these choices? Consciousness and Cognition, 16, 2-17.

Pierce, B.H., Sullivan, A.L., Schacter, D.L., \& Budson, A.E. (2005). Comparing source-based and gistbased false recognition in aging and Alzheimer's disease. NeURopsychologr, 19, 411-419.

Posner, M.I., \& Petersen, S.E. (1990). The attention system of the human brain. Annual Review of NeURosCIence, 13, 25-42.

Rabinowitz, F.M. (1970). Characteristic sequential dependencies in multiple-choice situations. Psychological Bulletin, 74, 141-148.

Radomsky, A.S., Gilchrist, P.T., \& Dussault, D. (2006). Repeated checking really does cause memory distrust. BEHAVIOUR RESEARCH AND THERAPY, 44, 305-316.

Rapcsak, S.Z., Polster, M.R., Comer, J.F., \& Rubens, A.B. (1994). False recognition and misidentification of faces following right hemisphere damage. CORTEX, 30,565-583.

Rapcsak, S.Z., Reminger, S.L., Glisky, E.L., Kaszniak, A.W., \& Comer, J.F. (1999). Neuropsychological mechanisms of false facial recognition following frontal lobe damage. Cognitive Neuropsychologr, 16, 267-292.

Rawlings, D., Claridge, G., \& Freeman, J.L. (2001). Principal components analysis of the schizotypal personality scale (STA) and the borderline personality scale (STB). Personality and Individual DIFFERENCES, 31, 409-419.

Read, J.D., \& Lindsay, D.S. (1997). Recollections of trauma: Scientific research and clinical practice. New York: Plenum Press.

Reinitz, M.T., \& Hannigan, S. (2004). False memories for compound words: Role of working memory. Memory and Cognition, 32, 463-473.

Reyna, V.F., \& Brainerd, C.J. (1995). Fuzzy-trace theory: An interim synthesis. LEARNING aNd INDIVIDUAL DIFFERENCES, 7, 1-75.

Roberts, K.P., \& Powell, M.B. (2005). The relation between inhibitory control and children's eyewitness memory. Applied Cognitive Psychology, 19, 1003-1018.

Robinson, K.J., \& Roediger, H.L.III. (1997). Associative processes in false recall and false recognition. Psychological SCIENCE, 8, 231-237.

Rodriguez-Fornells, A., Kofidis, C., \& Münte, T. (2004). An electrophysiological study of errorless learning. Cognitive Brain Research, 19, 160-173.

Roediger, H.L.III., Balota, D.A., \&Watson, J.M. (2001). Spreading activation and arousal of false memories. In H.L. Roediger, J.S. Nairne, I. Neath, \& A.M. Suprenant (Eds.), The NATURE of Remembering: Essays in honor of Robert G. CRowder (pp. 95-115). Washington DC: American Psychological Association.

Roediger, H.L.III., \& McDermott, K.B. (1995). Creating false memories: Remembering words not presented in lists. Journal of Experimental Psychology: Learning, Memory and Cognition, 21, 803-814.

Roediger, H.L.III., \& McDermott, K.B. (2000). Distortions of memory. In E. Tulving \& F.I.M. Craik (Eds.), THE OXFORD HANDBOOK OF MEMORY (pp. 149-162). NewYork: Oxford University Press.

Roediger, H.L.III., Meade, M.L., \& Bergman, E.T. (2001). Social contagion of memory. Psychonomic Bulletin AND REVIEW, 8, 365-371.

Roediger, H.L.III., Watson, J.M., McDermontt, K.B., \& Gallo, D.A. (2001). Factors that determine false recall: A multiple regression analysis. Psychonomic Bulletin and Review, 8, 385-407.

Rosenberg, S., Weber, N., Crocq, M.A., Duval, F., \& Macher, J.P. (1990). Random number generation by normal, alcoholic and schizophrenic subjects. Psychological MEDICINE, 20, 953-960.

Ruffman, T., Rustin, C., Garnham, W., \& Parkin, A.J. (2001). Source monitoring and false memories in children: Relation to certainty and executive functioning. Journal of Experimental Child Psychology, 80, 95-111.

Ryan, J.J., \& Paolo, A.M. (2001). Exploratory factor analysis of the WAIS-III in a mixed patient sample. Archives of Clinical Neuropsychology, 16, 151-156.

Ryan, J.J., Paolo, A.M., Miller, D.A, \& Morris, J. (1997). Exploratory factor analysis of the Wechsler adult intelligence scale-revised in a sample of brain-damaged women. ArCHIVES of Clinical NeURopsychology, 12, 683-689.

Rybash, J.M., Rubenstein, L., \& DeLuca, K.L. (1997). How to become famous but not necessarily recognizable: Encoding processes and study-test delays dissociate source monitoring from recognition. American Journal of Psychology, 110, 93-114. 
Sack, A.T., \& Linden, D.E.J. (2003). Combining transcranial magnetic stimulation and functional imaging in cognitive brain research: Possibilities and limitations. Brain RESEARCH REVIews, 43, 41-56.

Salamé, P. Danion, J.M., Peretti, S., \& Cuervo, C. (1998). The state of functioning of working memory in schizophrenia. SCHIZOPHRENIA RESEARCH, 30, 11-29.

Sanders, H.I., \& Warrington, E.K. (1971). Memory for remote events in amnesic patients. BraIN, 94, 661-668.

Schacter, D.L. (1999). The seven sins of memory: Insights form psychology and cognitive neuroscience. American Psychologist, 54, 182-203.

Schacter, D.L. (2001). The SEven sins of memory: How the mind forgets and remembers. New York: Houghton Mifflin Company.

Schacter, D.L., Buckner, R.L., Koutstaal, W., Dale, A.M., \& Rosen, B.R. (1997). Late onset of anterior prefrontal activity during retrieval of veridical and illusory memories: An event-related fMRI study. Neurolmage, 6, 259-269.

Schacter, D.L., Curran, T., Galluccio, L., Milberg, W.P., \& Bates, J.F. (1996a). False recognition and the right frontal lobe: A case study. Neuropsychologia, 34, 793-808.

Schacter, D.L., Koutstaal, W., \& Norman, K.A. (1997). False memories and aging. TRends in Cognitive SCIENCES, 1, 229-236.

Schacter, D.L., Norman, K.A., \& Koutstaal, W. (1998). The cognitive neuroscience of constructive memory. Annual Review of Psychology, 49, 289-318.

Schacter, D.L., Reiman, E., Curran, T., Yun, L.S., Bandy, D., McDermott, K.B., et al. (1996c). Neuroanatomical correlates of veridical and illusory recognition memory: Evidence from positron emission tomography. NeURon, 28, 1166-1172.

Schacter, D.L., Savage, C.R., Alpert, N.M. , Rauch, S.L., \& Albert, M.S. (1996b). The role of hippocampus and frontal cortex in age-related memory changes: A PET study. NeURoREPORT, 7, 1165-1169.

Schacter, D.L., \& Slotnick, S.D. (2004). The cognitive neuroscience of memory distortion. NEURON, 44, 149-160.

Schacter, D.L., Verfaellie, M., \& Koutstaal, W. (2002). Memory illusions in amnesic patients: Findings

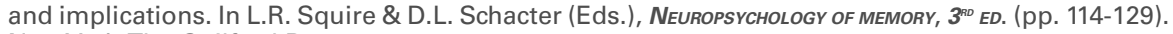
New York: The Guilford Press.

Schmand, B., Lindeboom, J., \& van Harskamp, F. (1992). Dutch adult reading test. Lisse, The Netherlands: Swets \& Zeitlinger.

Schneider, D.J. (2004). The psychology of Stereotyping. New York: The Guilford Press.

Schneider, S., Joppich, G., Lugt, van der A., Däuper, J., Münte, T.F. (2004). Brain potentials and selfpaced random number generation in humans. NeUROSCIENCE LETTERS, 367, 51-55.

Scoboria, A., Mazzoni, G., Kirsch, I, \& Relyea, M. (2004). Plausibility and belief in autobiographical memory. ApPlied Cognitive Psychology, 18, 791-807.

Seamon, J.G., Goodkind, M.S., Dumey, A.D., Dick, E., Aufseeser, M.S., Strickland, S.E., et al. (2003). “If I didn't write it, why would I remember it?": Effects of encoding, attention, and practice on accurate and false memory. Memory and Cognition, 31, 445-457.

Seidman, L.J., Yurgelun-Todd, D., Kremen, W.S., Woods, B.T., Goldstein, J.M., Faraone, S.V., et al. (1994). Relationship of prefrontal and temporal lobe MRI measures to neuropsychological performance in chronic schizophrenia. Biological Psychiatry, 35, 235-246.

Sherman, J.W., \& Bessenoff, G.R. (1999). Stereotypes as source-monitoring cues: On the interaction between episodic and semantic memory. PsYCHOLOGICAL SCIENCE, 10, 106-110.

Shimamura, A.P. (1995). Memory and the frontal lobe function. In M. Gazzaniga (Ed.), The cognitive NEURosCIENCES (pp. 803-813). Cambridge, MA: MIT Press.

Shinba, T., Shinozaki, T., Kariya, N., \& Ebata, K. (2000). Random number generation deficit in schizophrenia characterized by oral vs. written response modes. Perceptual and Motor Skills, 91, 1091-1105.

Smeets, T., Jelicic, M., Peters, M.J.V., Candel, I., Horselenberg, R., \& Merckelbach, H. (2006). “Of course I remember seeing that film!": How ambiguous questions generate crashing memories. APPLIED Cognitive Psychology, 20, 779-789.

Smeets, T., Merckelbach, H., Horselenberg, R., \& Jelicic, M. (2005). Trying to recollect past events: Confidence, beliefs, and memories. Clinical Psychology Review, 25, 917-934.

Snodgrass, J.G., \& Corwin, J. (1988). Pragmatics of measuring recognition memory: Applications to dementia and amnesia. Journal of Experimental Psychology: General, 117, $34-50$.

Spanos, N.P., Menary, E., Gabora, N.J., DuBreuil, S.C., \& Dewhirst, B. (1991). Secondary identity enactments during hypnotic past-life regression: A sociocognitive perspective. JourNAL OF PERSONALITY and Social Psychology, 61, 308-320.

Squire, L.R., \& Schacter, D.L. (2002). Neuropsychology of Memory, $3^{\text {ro }}$ ED. New York: The Guilford Press.

Stadler, M.A., Roediger, H.L.III., \& McDermott, K.B. (1999). Norms for word lists that create false memories. Memory and Cognition, 27, 494-500.

Stevens, J.P. (1992). Applied multivariate statistics for the social sciences, $2^{\text {no }}$ ed. Hillsdale, NJ: Erlbaum. 
Stinissen, J., Willems, P., Coetsier, P. \& Hulsman, W. (1970). Handleiding biJ de Nederlandstalige bewerking van de Wechsler Adult Intelligence Scale (WaIS). [Manual fo the Dutch version of the Wechsler Adult Intelligence SCALE (WAIS)] Lisse, The Netherlands: Swets en Zeitlinger.

Stroop, J. (1935). Studies of interference in serial verbal reactions. Journal of Experimental Psychology, 18, 643-662.

Toglia, M.P., Neuschatz, J.S., \& Goodwin, K.A. (1999). Recall accuracy and illusory memories: When less is more. Memory, 7, 233-256.

Towse, J.N. (1998). On random generation and the central executive of working memory. BRITISH JoURNAL of Psychologr, 89, 77-101.

Towse, J.N., \& Neil, D. (1998). Analyzing human random generation behaviour: A critical review of methods used and a computer program for describing performance. BeHAVIOR ResEARCH Methods, INSTRUMENTS \& COMPUTERS, 30, 583-591.

Towse, J.N., \& Valentine, J.D. (1997). Random generation of numbers: A search for underlying processes. European Journal of Cognitive Psychology, 9, 381-400.

Tranel, D., \& Damasio, H. (1994). Neuroanatomical correlates of electrodermal skin conductance responses. Psychophysiology, 31, 427-438.

Treisman, M., \& Faukner, A. (1987). Generation of random sequences by human subjects: Cognitive operations or psychophysical process? Journal of Experimental Psychology: General, 116, 337-355.

Troyer, A.K., \& Craik, F.I.M. (2000). The effect of divided attention on memory for items and their context. Canadian Journal of Experimental Psychology, 54, 161-170.

Tulving, E. (1985). Memory and consiciousness. CANAdian Psychologist, 26, 1-12.

Turner, M.L., \& Engle, R.W. (1989). Is working memory capacity task dependent? JourNal of MEMORY AND LANGUAGE, 28, 127-154.

Underwood, B.J. (1965). False recognition produced by implicit verbal responses. JouRNAL OF EXPERIMENTAL PsYchology, 70, 122-129.

Van Knippenberg, A., Dijksterhuis, A., Vermeulen, D. (1999). Judgment and memory of a criminal act: The effect of stereotypes and cognitive load. European Journal of Social Psychology, 29, 191-201.

Van der Linden, M., Beerten, A., \& Pesenti, M. (1998). Age-related differences in random number generation. BRAIN AND Cognition, 38, 1-16.

Van Loon - Vervoorn, W.A., \& Bekkum van, I.J. (1991). Woordassociatie LEXICON. [Word association LeXICON]. Amsterdam: Swets \& Zeitlinger.

Verfaellie, M., Page, K., Orlando, F., \& Schacter, D.L. (2005). Impaired implicit memory for gist information in amnesia. NeURopsychology, 19, 760-769.

Verhage, F. (1964). Intelligentie en LeeftiJd [INTElligence and age]. Assen, The Netherlands: Van Gorcum.

Vinogradov, S., Willis-Shore, J., Poole, J.H., Marten, E., Ober, B.A., \& Shenaut, G.K. (1997). Clinical and neurocognitive aspects of source monitoring errors in schizophrenia. American Journal of Psychiatry, 154, 1530-1537.

Von Hippel, W., Silver, L.A., \& Lynch, M.E. (2000). Stereotyping against your will:The role of inhibitory ability in stereotyping and prejudice among the elderly. Personality and Social Psychology Bulletin, 26, 523-532.

Wagenaar, W.A. (1970). Subjective randomness and the capacity to generate information. ACTA Psychologica, 33, 233-242.

Wagenaar, W.A. (1972). Generation of random sequences by human subjects: A critical survey of literature. Psychological Bulletin, 77, 65-72.

Wager, T.D., \& Smith, E.E. (2003). Neuroimaging studies of working memory: A meta-analysis. CogNitIVE, affective, and Behavioral Neuroscience, 3, 255-274.

Waldie, B.D., \& Kwong See, S.T. (2003). Remembering words never presented: False memory effects in dementia of the Alzheimer type. Aging, Neuropsychology, and Cognition, 10, 281-297.

Ward, J., Parkin, A.J., Powell, G., Squires, E.J., Townshend, J., \& Bradley, V. (1999). False recognition of unfamiliar people: "seeing film stars everywhere". Cognitive NeuRopsychology, 16, 293-315.

Waters, F.A.V., Maybery, M.T., Badcock, J.C., \& Michie, P.T. (2004). Context memory and binding in schizophrenia. SCHIZOPHRENIA RESEARCH, 68, 119-125.

Watkins, E., \& Brown, R.G. (2002). Rumination and executive function in depression: An experimental study. Journal of Neurology, Neurosurgery and Psychiatry, 72, 400-402.

Watson, D. (2001). Dissociation of the night: Individual differences in sleep-related experiences and their relation to dissociation and schizotypy. Journal of Abnormal Psychology, 110, 526-535.

Watson, J.M., Balota, D.A., \& Sergent-Marshall, S.D. (2001). Semantic, phonological, and hybrid veridical and false memories in healthy older adults and in individuals with dementia of the Alzheimer type. NeURopsychology, 15, 254-267.

Watson, J.M., Bunting, M.F., Poole, B.J., \& Conway, A.R.A. (2005). Individual differences in susceptibility to false memory in the Deese-Roediger-McDermott paradigm. Journal of Experimental Psychology: Learning, Memory, and Cognition, 31, 76-85.

Watson, J.M., McDermott, K.B., \& Balota, D.A. (2004). Attempting to avoid false memories in the Deese/ Roediger-McDermott paradigm: Assessing the combined influence of practice and warnings in young and old adults. Memory and Cognition, 32, 135-141. 
Wechsler, D. (1997). Administration and scoring manual for the Wechsler Adult Intelligence Scale, $3^{\text {id }}$ ed. San Antonio, TX:The Psychological Corporation.

Weinberger, D.R., Egan, M.F., Bertolino, A., Callicott, J.H., Mattay, V.S., Lipska,B.K., et al. (2001). Prefrontal neurons and the genetics of schizophrenia. Biological Psychiatry, 50, 825-844.

Weinstein, E.A. (1996). Symbolic aspects of confabulation following brain injury: Influence of premorbid personality. Bulletin of the MenNinger Clinic, 60, 331-350.

Weiss, A.P., Dodson, C.S., Goff, D.C., Schacter, D.L., \& Heckers, S. (2002). Intact suppression of increased false recognition in schizophrenia. American Journal of Psychiatry, 159, 1506-1513.

Wegner, D.M., Schneider, D.J., Carter, S.R., \& White, T.L. (1987). Paradoxical effects of thought suppression. Journal of Personality and Social Psychology, 53, 5-13.

Wenzlaff, R.M., \& Wegner, D.M. (2000). Thought suppression. Annual Review of Psychology, 51, 59-91.

Wheeler, M.A., Stuss, D.T., \& Tulving, E. (1997). Toward a theory of episodic memory: The frontal lobes and autonoetic consciousness. Psychological Bulletin, 121, 331-354.

Williams, M.A., Moss, S.A., Bradshaw, J.L., \& Rinehart, N.J. (2002). Random number generation in autism. Journal of Autism and Developmental Disorders, 32, 43-47.

Wilson, B.A., Alderman, N., Burgess, P.W., Emslie, H.E., \& Evans, J.J. (1996). Behavioural assessment of the dyseXecutive syndrome. Bury St Edmunds, UK: Thames ValleyTest Company.

Wilson, S.C., \& Barber, T.X. (1982). Fantasy-prone personality: Implications for understanding imagery, hypnosis, and parapsychological phenomena. PSI-RESEARCH, 1, 94-116.

Wilson, B.A., Evans, J.J., Emslie, H., Alderman, N., \& Burgess, P. (1998). The development of an ecologically valid test for assessing patients with a dysexecutive syndrome. NeUROPSYCHOLOGICAL REHABILITATION, 8, 213-228.

Winograd, E., Peluso, J.P., \& Glover, T.A. (1998). Individual differences in susceptibility to memory illusions. Applied Cognitive Psychology, 12, S5-S27.

Woll, S.B., \& Graesser, A.C., (1982). Memory discrimination for information typical or atypical of person schemata. Social Cognition, 1, 287-310.

Wood, J.N., Romero, S.G., Knutson, K.M., \& Grafman, J. (2005). Representation of attitudinal knowledge: Role of prefrontal cortex, amygdala and parahippocampal gyrus. NeURopsYCHologiA, 43, 249-259.

Wright, D.B., \& Osborne, J.E. (2005). Dissociation, cognitive failures, and working memory. AMERICAN Journal of Psychology, 118, 103-113.

Wright, D.B., Startup, H.M., \& Mathews, S.A. (2005). Mood, dissociation and false memories using the Deese-Roediger-McDermott procedure. British Journal of Psychologr, 96, 283-293.

Wyland, C.L., Kelley, W.M., Macrae, C.N., Gordon, H.L., \& Heatherton, T.F. (2003). Neural correlates of thought suppression. NeURopsychologia, 41, 1863-1867.

Young, A.W., Flude, B.M., Hay, D.C., \& Ellis, A.W. (1993). Impaired discrimination of familiar from unfamiliar faces. CORTEX, 29, 65-75.

Zeelenberg, R., \& Pecher, D. (2002). False memories and lexical decision: Even twelve primes do not cause long-term semantic priming. АстA PsychologicA, 109, 269-284.

Zeelenberg, R., Plomp, G., \& Raaijmakers, J.G.W. (2003). Can false memories be created through nonconscious processes? ConsCIOUSNESS AND COGNITION, 12, 403-412.

Zoccolotti, P., Scabini, D., \& Violani, C. (1982). Electrodermal responses in patients with unilateral brain damage. Journal of Clinical Neuropsychology, 4, 143-150. 


\section{[S]UMMARY}

Our memory is reconstructive in nature. Sooner or later, a good number of our experiences will be forgotten, and the events we do remember, may differ from how they actually took place. Often, there are only minor discrepancies between actual events and our memories (i.e., memory distortions), but sometimes we remember events that never happened in reality. In such cases, people are said to have pseudomemories.

In recent years, a vast amount of research has explored whether certain personality traits may be related to source misattributions and pseudo-memories. However, the connection between these traits and memories for events that did not take place is far from robust. In this respect, findings in neuropsychological research could give us a better clue on individual differences in the development of pseudo-memories. Up until now aging and lesion studies suggest that neurocognitive dysfunctions play a critical role in the creation of memories for events that did not happen. Therefore, focusing on neurocognitive concepts that may relate to pseudo-memories might be more fruitful. Given the considerable amount of aging and clinical research, it is surprising how few studies have explored whether individual differences in neurocognitive tasks in healthy adults influence vulnerability to pseudo-memories. Also, specific questions remain open to debate on the relationship between neurocognitive functions and pseudo-memories in specific clinical samples. These are the central themes inspiring the studies presented in this dissertation.

Part $A$ of this dissertation is devoted to the validation of a pseudo-memory paradigm and neurocognitive task. Chapter 2 describes the construction, validation, and standardization of a Dutch version of the Deese/Roediger-McDermott paradigm (DRM paradigm; Deese, 1959b; Roediger \& McDermott, 1995) in an undergraduate sample. Briefly, in this paradigm participants are given lists of semantically related words (e.g., bed, pajama) that refer to a non-presented critical lure word (e.g., sleep). In subsequent memory tests, a vast amount of participants claim to remember these non-presented critical lure words. Results of this study show that although all lists were constructed in a similar way (on the basis of Dutch word association norms), rates of falsely recalling and recognizing the critical lure words varied widely across lists. Furthermore, participants often recognized critical lures with high confidence, as indicated by the "remember" responses for critical lure intrusions.

Chapter 3 reports the results of four studies on the psychometric properties of the Random Number Generation task (RNG; Ginsburg \& Karpiuk, 1994, 1995), a neurocognitive task measuring monitoring, updating, and inhibition function. The factor structure of the RNG is best described by a three factor solution (Study 1): Seriation, repetition, and cycling related to inhibition of stereotypical schemas, output inhibition, and monitoring of previous output, respectively. Study 2 examines the test-retest reliability of these RNG factors in a sample of undergraduate students and schizophrenic patients. We found modest to satisfactory test-retest correlations and 
no influence of practice, with RNG indices loading on the seriation factor showing satisfactory stability across time. Studies 3 and 4 describe results on criterion and construct validity of the RNG task. The results indicate that RNG has modest to acceptable psychometric properties and primarily taps inhibition, updating and monitoring functions in normal as well as clinical populations.

The studies described in Part B focus on the relationships between suboptimal neurocognitive functioning, source misattributions, and experimentally induced pseudo-memory development in healthy undergraduate participants. The study described in Chapter 4 shows that in our sample of undergraduates, individual differences in inhibitory function as measured by the RNG task (see Chapter 3 ) are related to false recognition and to a lesser extent to false recall of non-presented critical lure words in the DRM paradigm.

Chapter 5 elaborates on these findings. The two studies described in this Chapter investigate whether individual differences in simple and complex Working Memory Capacity (WMC) are related to false recall and recognition of non-presented critical lures. The two studies converge on the notion that poor simple WMC (as indexed by suboptimal backward digit span) is related to false recognition and, to a lesser extent false recall of critical lures. Complex WMC did not relate to false recall or recognition of the critical lure words.

Because this WMC - pseudo-memory link warranted further study, Chapter 6 in part examines whether complex WMC is related to source misattributions in a high executive demand environment. In this study, undergraduates are subjected to an action source monitoring paradigm, in which to-be-remembered acts have to be performed, while others only have to be imagined, thereby putting increased executive demands on WMC. Subsequently, the presented actions are paired with actions that are roughly similar in content and form. For each pair, old-new discrimination (i.e., correct recognition) and source attribution (having performed or only imagined the action) decisions have to be made. In line with the findings of Watson and colleagues (2005), we found that poor complex WMC is related to source misattributions in a high executive demand environment (e.g., claiming having performed an action while, in fact, it is only imagined).

Chapter 7 describes a study in which we investigated whether an active manipulation of inhibitory control leads to an increased number of source misattributions and subsequent pseudo-memories for general schematic knowledge. More specifically, in this study people did or did not receive an instruction to actively inhibit their thoughts on activated neutral or negative stereotypes about race, while reading a stereotypefree crime story. We anticipated that such active inhibition would lead to a decrease in focus on the source of information during encoding due to increased executive demands. During later retrieval, one will more likely rely on primed general schematic knowledge, thereby misattributing non-presented general schematic knowledge as being presented. Our findings, indeed, supported this line of reasoning. 
Part $\mathrm{C}$ of this dissertation describes two studies addressing the relationship between neurocognitive dysfunctions, source misattributions, and pseudo-memories in clinical samples. In the study described in Chapter 8, we investigated whether executive dysfunctions could explain certain memory aberrations in schizophrenic patients. It was found that, relative to matched controls, patients with schizophrenia make less correct recognitions. As to pseudo-memories, both groups showed a substantial number of pseudo-memories for critical lures. However, it was the control group that had the highest level of false recognition. For source misattribution, our findings concur with previous research in that schizophrenic patients, compared to healthy controls, were more likely to make internal-external source misattributions with inflated confidence ratings. The executive functioning indexes were significant predictors of correct recognition, but not of source misattributions or pseudo-memories in schizophrenic patients.

An important question in investigating the relationship between neurocognitive functioning, pseudo-memories, and source misattributions is whether such misattributions may also occur for highly implausible events. This issue is studied in Chapter 9, in which participants with highly implausible autobiographical beliefs and memories (hypnotically induced previous-life beliefs and memories) are compared to matched controls who do not report such highly implausible events on their susceptibility to make source misattributions. Results show that previous-life participants are more susceptible to these misattributions in comparison to matched control participants. Our findings suggest that subjective rather than objective plausibility appears to be a necessary factor in the cascade of processes that lead to full blown pseudo-memories.

Finally, in Chapter 10, the results of our work are summarized, discussed, an integrated theoretical framework is presented, and some final conclusions are drawn. To further illustrate our findings, some new data on the link between pseudo-memory and neurocognitive functions are briefly presented. Directions for future research are also discussed. 


\section{[S]AMENVATTING}

Onze herinneringen zijn geen exacte kopie van een feitelijke gebeurtenis, maar reconstructies van deze gebeurtenis. Door deze reconstructie kunnen we ons zaken anders gaan herinneren dan hoe zij zich in werkelijkheid hebben voorgedaan. Dit kan variëren van kleine vervormingen (bv. ik herinner me een blauwe taxi terwijl de taxi geel was) tot het herinneren van dingen die nooit hebben plaatsgevonden (je herinnert je dat je gisteren een vriend bent tegengekomen in de stad, terwijl dit in werkelijkheid niet zo was). In een dergelijk geval spreken we van een pseudo-herinnering.

De belangstelling voor onderzoek naar pseudo-herinneringen heeft zich in de afgelopen jaren sterk gericht op de vraag wie de mensen zijn die zulke "herinneringen" ontwikkelen. Helaas is de zoektocht naar persoonlijkheidstrekken als voorspeller van herinneringen voor gebeurtenissen die zich niet hebben voorgedaan nogal teleurstellend geweest. Recent neuropsychologisch onderzoek bij ouderen en klinische groepen suggereerde een neuropsychologische verankering van pseudoherinneringen. Ofschoon er veel onderzoek is uitgevoerd naar de relatie tussen pseudo-herinneringen en neurocognitieve disfuncties bij ouderen, patiënten met hersenschade en klinische stoornissen, heeft er nauwelijks onderzoek plaatsgevonden naar de neuropsychologische determinanten van zulke "herinneringen" bij gezonde volwassenen. Bovendien blijft een aantal vragen rond de relatie tussen neurocognitieve functies en pseudo-herinneringen in klinische groepen onbeantwoord. Deze onderwerpen vormen de centrale thema's in dit proefschrift en worden nader beschreven in Hoofdstuk 1.

In deel A staat de validatie van een pseudo-herinnering paradigma en een neurocognitieve taak centraal. Hoofdstuk 2 beschrijft de standaardisatie van een $\mathrm{Ne}-$ derlandse versie van het Deese/Roediger-McDermott paradigma (DRM paradigma; Deese, 1959b; Roediger \& McDermott, 1995). Kort gezegd komt dit paradigma hierop neer: proefpersonen krijgen een lijst van woorden aangeboden (bv. bed, pyama) die semantisch gerelateerd zijn aan een niet gepresenteerd woord (bv. slaap), welke het kritische woord wordt genoemd. Wanneer hen later gevraagd wordt de gepresenteerde woorden te herinneren, komt een substantieel deel van de proefpersonen met het kritische niet-gepresenteerde woord op de proppen. De resultaten van onze studie tonen aan dat, hoewel al de woordenlijsten op een vergelijkbare manier zijn geconstrueerd, ze grote verschillen vertonen m.b.t. het uitlokken van het kritische woord. Als later aan de proefpersonen wordt gevraagd of ze hier een echte herinnering aan hebben, geeft men in de regel aan dat hun herinneringen waarheidsgetrouw zijn.

In Hoofdstuk 3 worden de resultaten besproken van 4 studies naar de psychometrische aspecten van de Random Number Generation taak (RNG; Ginsburg \& Karpiuk, 1994; 1995). De RNG is een neurocognitieve test die "monitoring", "updating", en "inhibitie" functies meet. Studie 1 beschrijft een drie factoren structuur voor de RNG taak: "seriation", "repetition" en "cycling". Deze factoren zijn gerelateerd aan 
inhibitie van stereotype schema's, output inhibitie, en monitoren van voorgaande output. In Studie 2 wordt de test-hertest betrouwbaarheid van deze RNG factoren beschreven in een groep van studenten en schizofrene patiënten. We vonden een bescheiden tot bevredigende test-hertest correlatie. Studies 3 en 4 beschrijven criterium en construct validiteit. Op basis van deze resultaten kan geconcludeerd worden dat de RNG over matige tot acceptabele psychometrische eigenschappen beschikt, en primair inhibitie, updating en monitoring functies meet in zowel normale als klinische groepen.

Deel B van dit proefschrift focust zich op onderzoek naar de relatie tussen neurocognitieve functies, bronverwarring, en experimenteel uitgelokte pseudo-herinneringen bij een groep van gezonde volwassenen. In Hoofdstuk 4 wordt ondersteuning gevonden voor een relatie tussen individuele verschillen in inhibitie functie en foutieve herkenning en, in mindere mate, herinnering van het niet gepresenteerde kritische woord in het DRM paradigma.

Hoofdstuk 5 bouwt voort op de bevindingen van Hoofdstuk 4. De twee studies beschreven in dit hoofdstuk zoeken ondersteuning voor de vraag of individuele verschillen in werkgeheugen capaciteit (simpel vs. complex) gerelateerd zijn aan experimenteel uitgelokte pseudo-herinneringen in het DRM paradigma. De resultaten van deze studies tonen aan dat onder beperkte cognitieve belasting, suboptimale scores op een simpele werkgeheugentaak (achterwaartse digit span) gerelateerd zijn aan foutieve herkenning van het kritische woord en, in mindere mate, foutieve herinnering van het kritische woord.

In Hoofdstuk 6 vindt de lezer een empirische toetsing van de hypothese dat bronverwarringsfouten, in situaties waarbij proefpersonen cognitief belast worden, gerelateerd zijn aan beperkingen in complexe werkgeheugen capaciteit. Deze hypothese is ontleend aan onderzoek van Watson en collega's (2005). Aan de proefpersonen wordt gevraagd specifieke acties (bv. breek een tandenstoker in tweeën) in te beelden of uit te voeren. Vervolgens worden deze gepresenteerde acties gepaard aangeboden met acties die grotendeels overeenkomen wat betreft inhoud en vorm (bv. breek een tandenstoker in drieën). Voor elk paar moet de proefpersoon aangeven welke handeling oud of nieuw is en moet bovendien worden aangegeven of hij/zij de oude handeling zich slechts heeft ingebeeld of daadwerkelijk heeft uitgevoerd. De resultaten blijken de hypothese te ondersteunen.

Hoofdstuk 7 beschrijft een studie waarbij proefpersonen expliciet de boodschap kregen gedurende een bepaalde tijd niet te denken aan algemene stereotype schema's rond etniciteit, geslacht en leeftijd (actieve inhibitie). Dit moest gebeuren tijdens het lezen van een misdaadverhaal dat stereotype neutraal was. Naar aanleiding van eerder onderzoek (bv. Araya et al., 2003) verwachtten we dat door deze actieve inhibitie van schema informatie, de broninformatie van het verhaal suboptimaal opgeslagen wordt. Tijdens het ophalen van die informatie zullen proefpersonen echter paradoxaal meer gaan vertrouwen op die algemene stereotype schema's die 
ze eerst moesten onderdrukken wat op zich dan leidt tot een toename in bronverwarring fouten tijdens een herkenningstaak. De resultaten ondersteunden deze redenering.

Deel $C$ van het proefschrift richt zich op de relatie tussen neurocognitieve disfuncties, het toeschrijven van broninformatie, en pseudo-herinneringen bij klinische groepen. In Hoofdstuk 8 wordt een studie beschreven waarbij gekeken wordt naar de relatie tussen uitvoerende controle disfuncties en geheugen afwijkingen (bronverwarring, pseudo-herinneringen, etc.) bij schizofrene patiënten. De resultaten tonen aan dat schizofrene patiënten slechter zijn in het correct herkennen van woorden in vergelijking met gezonde controles. Verder vertonen zulke patiënten een verhoogde neiging om dingen die door henzelf gezegd zijn toe te schrijven aan iemand anders. Uitvoerende controle disfuncties bij schizofrene patiënten waren met name verbonden met correcte herkenning van stimulusmateriaal, en niet met bronverwarring of pseudo-herinneringen.

Tot nog toe is er voornamelijk onderzoek uitgevoerd naar de relatie tussen neurocognitieve functies, bronverwarring en pseudo-herinneringen voor waarschijnlijke gebeurtenissen, zoals woordenlijsten, simpele acties, misdaadverhalen. Een vraag die echter gesteld moet worden is of personen ook pseudo-herinneringen kunnen ontwikkelen voor gebeurtenissen die zeer bizar en onwaarschijnlijk zijn. Deze vraag is onderwerp van studie in Hoofdstuk 9. In deze studie wordt de gevoeligheid voor het maken van bronverwarring fouten onderzocht bij proefpersonen die herinneringen hebben aan voorgaande levens en controleproefpersonen zonder zulke onwaarschijnlijke herinneringen. De resultaten van deze studie laten zien dat personen met zulke bizarre herinneringen meer vatbaar zijn voor het maken van bronverwarring fouten in vergelijking met de andere groep.

Ten slotte wordt in Hoofdstuk 10 eerst een samenvatting gegeven van de bevindingen. Deze worden ook nader bediscussieerd en geïntegreerd in een algemeen theoretisch raamwerk. $\mathrm{Na}$ het beschrijven van een aantal resultaten uit nieuwe studies, wordt dit hoofdstuk beëindigd met een bespreking van de klinische implicaties van het gepresenteerde onderzoek. 


\section{[DANKWOORD]}

Aan het einde van deze AIO rit wil ik toch even een pas op de plaats maken en een aantal mensen de revue laten passeren die erg van betekenis zijn geweest in de afgelopen jaren en drie decennia van mijn leven. Bij wijze van rode draad doorheen dit proefschrift, een dankwoord associatie lijst

Promotor Harald: uitstekend, leermeester 1, erudiet, glashelder, scherpe pen, rood, eminent, kritisch, deskundige, Limburger, bereikbaar, tijd, inspiratie, creatief.

Co-promotor Marko: uitstekend, leermeester 2, wetenschapper, deskundig, kritisch, open deur, wetenswaardigheden, geduld, kalmte, casuïstiek, Vlaamsche taal, bereikbaar, hey..., maar...

Rudolf: leermeester 3, praktijk, NPO, spontaan, bereikbaar, housesitting, vertrouwen, samenwerking, evidence-based, clinicus, onderzoeker, neuropsychologie, geheugen, deskundig.

IRP club: tofste, gezelligheid, collegialiteit, koffie, ernst, ontspanning, brainstormen, Ardennen, samenwerking, belangstelling, deskundig, psychologie, recht.

Collega's van CPS (formerly known as EP): tofste, vrijheid, ontspannen, gezelligheid, Wetenschap, ernst, openheid.

Paranimf (ex-kamergenoot) Tom: gezellig, betrouwbaar, vertrouwen, gesprekken, Belg, bibliotheek, archiveren, autoperikelen, empirist, scherpe pen, cynisme, bescheiden.

Kamergenoten Henry en Elke: gezelligheid, enthousiasme, open, ontspannen, muziek, talent, brainstorm, koffie, thee, onderzoeksperikelen, grappen, steun.

Proefpersonen, stagiaires, studen-assistenten, collega's van buiten de faculteit (Annelien Duits, Paul Vanhoorne, Dr. Bryon, Dr. Mesotten, Michael Hilderson): DANK

Vrienden (Sissi, Steven, Geoffrey en Christine, Jeffrey, Ronnie en Elke, ...): steun, relativering, filosoferen, gesprekken, onnozelheid, realiteit, jeugdsentiment, festivals, fuiven, geduld.

Ruth en Kurt: relativering, etentjes, film, ontspanning, therapeut, graficus, huis, bouwen, huismus ;) , creatief, roddel, neurotisch, luisteren. 
Zus, schoonbroer (en paranimf) en kleine Nore: welkom, relativering, interesse, ontspanning, blij, open, ta, no, knotsgek ;) , zondag, spaghetti, gezellig, liefde, vriendschap, (t)huis, oogappel.

Ria, Albert, oma Lisa, José, Eddy, oma, en overige familieleden: steun, vertrouwen, onbetaalbaar, open, interesse, ontspanning, gezelligheid, (t)huis.

Ma en pa: dankbaar, steun, vertrouwen, geduld, studeren, mogelijkheden, liefde, geluk, open, oma, opa, bereikbaar, spaghetti, zondagen, klaarstaan, bellen, opjagen ;) , (T)HUIS.

Ilse: steun, Isil, toeverlaat, vriendschap, liefde, alles, good times, bad times, geven, nemen, relativering, geduld, THUIS

There is always anyone anywhere, but no one like you 


\section{[G]URRICULUM [V]ITAE}

Maarten Peters werd geboren op 10 mei 1979 inTongeren, België. In juni 1997 behaalde hij zijn diploma algemeen secundair onderwijs aan het Onze-Lieve-Vrouwecollege in Tongeren. In dat zelfde jaar begon hij zijn studie Gezondheidswetenschappen, afstudeerrichting Geestelijke Gezondheidkunde, aan de Universiteit Maastricht. Na een jaar van individueel keuze onderwijs aan de Faculteit der Psychologie (20002001) en een praktijk- en onderzoeksstage binnen de geheugenpoli van het Academisch ziekenhuis Maastricht (2001-2002) rondde hij in september 2002 zijn studie af met het behalen van een doctoraal diploma. Van 2002 tot 2003 was hij werkzaam als onderzoeksassistent bij de vakgroep Psychiatrie en Neuropsychologie van het Academisch ziekenhuis Maastricht. In maart 2003 maakte hij zijn overstap naar de capaciteitsgroep Experimentele Psychologie van de Faculteit der Psychologie, waar hij aangesteld werd als Assistent in Opleiding (AIO). Momenteel is hij werkzaam als Post-Doc bij diezelfde vakgroep.

Maarten Peters was born in Tongeren (Belgium) on May 10th 1979. In June 1997 he graduated from secondary school (Onze-Lieve-Vrouwecollege,Tongeren). In September of that year, he started studying Mental Health Sciences at Maastricht University. After a year of electives at the faculty of Psychology (specialization Neuropsychology) and an applied training period at the Memory Clinic of the Academic Hospital of Maastricht, he graduated in September 2002, with a specialization in Mental Health Sciences. From 2002 until February 2003, he worked as a research assistant at the department of Psychiatry and Neuropsychology of the Academic Hospital of Maastricht. In March 2003 he started working as a PhD student at the Department of Experimental Psychology of the Faculty of Psychology, Maastricht University. He currently holds a Postdoctoral Fellow at the same department. 



\section{[LIST OF PUBLICATIONS]}

\section{International journal articles}

Arts, B. Peters, M.J.V., Ponds, R., Honig, A., \& Os van J. (2006). S 100-beta and impact of ECT on depression and cognition. JourNaL OF ECT, 22, 206-212.

Jelicic, M., Smeets, T., Peters, M., Candel, I., Horselenberg, R., \& Merckelbach, H. (2006). Assassination of a controversial politician: Remembering details from another non-existent film. Applied Cognitive Psychology, 20, 591-596.

Merckelbach, H., Peters, M.J.V., Jelicic, M., Brands, I., \& Smeets, T. (2006). Detection of malingering of Ganser-like symptoms with tests: A case study. PsYCHIATRY AND Clinical Neurosciences, 60, 636-638.

Pelsers, M.M., Hanhoff, T., Van Der Voort, D., Arts, B., Peters, M., Ponds, R., Honig, A., Rudzinski, W., Spener, F., De Kruijk, J.R., Twijnstra, A., Hermens, W.T., Menheere, P.P.C.A., \& Glatz, J.F.C. (2004). Brain- and heart- type fatty acid-binding proteins in the brain: Tissue distribution and clinical utility. Clinical Chemistry, 50, 15681575.

Peters, M.J.V., Cima, M., Smeets, T., de Vos, M., Jelicic, M., \& Merckelbach, H. (pending REVISION). Did I say that word or did you? Executive dysfunctions in schizophrenic patients affect memory efficiency, but not source attributions.

Peters, M.J.V., Giesbrecht, T., Jelicic, M., \& Merckelbach, H. (ACCEPTED). The Random Number Generation task: Psychometric properties and normative data of an executive function task in a mixed sample. Journal of the INTERNATIONAL Neuropsychological Society.

Peters, M.J.V., Horselenberg, R., Jelicic, M., Merckelbach, H. (2007). The false fame illusion in people with memories about a previous life. ConsciousNesS AND Cognition, 16, 162-169.

Peters, M.J.V., Jelicic, M., Haas, N., \& Merckelbach, H. (2006). Mild executive dysfunctions in undergraduates are related to recollecting words never presented. INTERnational Journal of Neuroscience, 116, 1065-1077.

Peters, M.J.V., Jelicic, M., \& Merckelbach, H. (2006). Neuropsychology and pseudomemories. In J.R. Dupri (Ed.), Focus on NEUROPSYCHOLOGY RESEARCH (pp. 163-184). Hauppauge, NY: NOVA Science Publishers.

Peters, M.J.V., Jelicic, M., \& Merckelbach, H. (2006). When stereotypes backfire: Trying to suppress stereotypes produces false recollections of a crime. LEGAL AND Criminological Psychology, 11, 327-336.

Peters, M.J.V., Jelicic, M., Op Heij, C.D.H., \& Merckelbach, H. (2006). Detection of malingered burnout using standard clinical questionnaires. German Journal of PSYCHIATRY, 9, 10-16.

Peters, M.J.V., Jelicic, M., Verbeek, H., \& Merckelbach, H. (2007). Poor working memory predicts false memories. European Journal of Cognitive Psychology, 19, 213-232.

Peters, M.J.V., Smeets, T., Giesbrecht, T., Jelicic, M., \& Merckelbach, H. (ACCEPTED). Confusing action and imagination: Action source monitoring in individuals with schizotypal traits. Journal of Nervous and Mental Disease.

Smeets, T., Jelicic, M., Peters, M.J.V., Candel, I., Horselenberg, R., \& Merckelbach, H. (2006). "Of course I remember seeing that film!" - How ambiguous questions generate crashing memories. Applied Cognitive Psychology, 20, 779-789.

Smeets, T., Jelicic, M., Merckelbach, H., Peters, M., Fett, A., Taverniers, J., Henquet, C., \& Dautzenberg, J. (2006). Enhanced memory performance on an internalinternal source monitoring task following acute psychosocial stress. BEHAVIORAL NeURoscience, 120, 1204-1210. 


\section{National journal articles}

Jelicic, M., Merckelbach, H., Peters, M.J.V. (2004).That's what we call a psychogenic amnesia. TIJDSCHRIFT VOoR PSYCHIATRIE, 46, 627-631.

Merckelbach, H., Smeets, T., Peters, M., \& Jelicic, M. (2005). Op weg naar een valse bekentenis: een schizofrene patiënt wordt verhoord. MaANdblad GeesteliJKe VOLKSGEZONDHEID, 60, 827-843.

Merckelbach, H., Jelicic, M., Peters, M., Brands, I., \& Smeets, T. (2006). Hoe Ganser man zijn basketbalteam naar de overwinning voerde. MAANDBLAD GEESTELIJKE VolKSGEZONDHEID, 61,614-627.

Peters, M.J.V., Ponds, R.W.H.M., Ham, van den P., Scheltens, Ph., \& Verhey, F.R.J. (2004). De 7 minuten test ter detectie van Dementie van het Alzheimertype: psychometrische aspecten. TiJdschrift voor Gerontologie en Geriatrie, 35, 114120.

Peters, M.J.V., Jelicic, M., Horselenberg, R., \& Merckelbach, H.(2004). De neuropsychologie van pseudoherinneringen. Neuropraxis, 8, 97-103.

\section{Submitted}

Jelicic, M., Peters, M.J.V., Leckie, V., \& Merckelbach, H. (sUвMıтTED). Basic knowledge of psychopathology does not undermine the efficacy of the Structured Inventory of Malingered Symptomatology (SIMS) to detect feigned psychosis.

Peters, M.J.V., Jelicic, M., \& Merckelbach, H. (sUBMIтTED). Inducing false memories: A Dutch version of the Deese/Roediger-McDermott paradigm.

Merckebach, H., Peters, M.J.V., Jelicic, M., \& Brands, I. (sUBMITTED). “Gaat het weer een beetje Al Capone?" Politie meets CVA patiënt. 